\title{
A risk management approach to pillar extraction in the Central Appalachian coalfields
}

\author{
Patrick R. Bucks \\ West Virginia University
}

Follow this and additional works at: https://researchrepository.wvu.edu/etd

\section{Recommended Citation}

Bucks, Patrick R., "A risk management approach to pillar extraction in the Central Appalachian coalfields" (2010). Graduate Theses, Dissertations, and Problem Reports. 2158.

https://researchrepository.wvu.edu/etd/2158

This Thesis is protected by copyright and/or related rights. It has been brought to you by the The Research Repository @ WVU with permission from the rights-holder(s). You are free to use this Thesis in any way that is permitted by the copyright and related rights legislation that applies to your use. For other uses you must obtain permission from the rights-holder(s) directly, unless additional rights are indicated by a Creative Commons license in the record and/ or on the work itself. This Thesis has been accepted for inclusion in WVU Graduate Theses, Dissertations, and Problem Reports collection by an authorized administrator of The Research Repository @ WVU. For more information, please contact researchrepository@mail.wvu.edu. 
A Risk Management Approach to Pillar Extraction in the Central Appalachian Coalfields

Patrick R. Bucks

Thesis submitted to the College of Engineering and Mineral Resources at West Virginia University in partial fulfillment of the requirements

for the degree of

Master of Science

in

Mining Engineering

Christopher J. Bise, Ph.D., Chair

Keith A. Heasley, Ph.D.

Yi Luo, Ph.D

Christopher Mark, Ph.D

Daniel Della-Giustina, Ph.D

Department of Mining Engineering

Morgantown, West Virginia

2010

Keywords: Coal Pillar Extraction, Retreat Mining, Central Appalachia, Mining Engineering, Risk Management, Coal Mine Safety 


\section{ABSTRACT \\ A Risk Management Approach to Pillar Extraction in the Central Appalachian Coalfields}

Patrick R. Bucks

Central Appalachian coal reserves are a valuable natural resource in high demand, both domestically and internationally. The need for this resource necessitates recovery a high percentage of the coal reserves. The mining technique that is most often utilized to extract Central Appalachian coal at high recovery rates involves the development of entries and cross-cuts within the coal seam and the subsequent removal of the coal pillars that remain. The practice of extracting coal pillars is commonly referred to as pillaring or retreat mining.

The problem associated with pillar extraction is the additional safety risk posed to miners during pillar extraction. This thesis, will investigate the application of pillaring in Central Appalachia, and based on this investigation, develop a risk management-based strategy that targets personnel performance-based risk, in order to improve the ability of safety trainers to tailor training programs to the needs of pillaring sections.

Several conclusions have been determined in this thesis. Although pillaring has its roots in antiquated mining methods, current technology and mining practices have made this form of mining highly modernized. This modernization of the pillaring process has made great strides towards improving safety. However, current fatality trends suggest that work still needs to be done to control the residual risk that is not controlled by modern machinery and mining practices. Fatalities and accidents almost always have a personnel performance related component. These performances that have been identified in previous fatal accidents are still commonly observed in Central Appalachia. The risk management based strategy developed in this thesis can successfully identify and quantify risk associated with personnel performance. This ability to quantitatively assess performance based risk can have a significant impact on the ability of safety managers to train the workforce, and therefore, should have a positive impact on pillaring safety in Central Appalachia. 


\section{ACKNOWLEDGEMENTS}

The author of this thesis would like to express his sincere appreciation to those who have contributed to the contents of this thesis. Particular thanks go to:

- Professor Dr. Christopher J. Bise, advisor and committee chair, for his guidance, encouragement, assistance, and advice during the development of this thesis, Dr. Bise spent countless hours proofreading and guiding the development of this thesis and for that I am sincerely grateful

- The West Virginia University, Department of Mining Engineering and the National Institute for Occupational Safety and Health (NIOSH) for financial support of this research through contract 200-2008-24752 with Dr. Christopher Mark Serving as the Technical Project Officer,

- The mining companies, which, graciously, allowed case studies and field applications to be conducted at their facilities, and

- Dr. Keith Heasley, Dr. Daniel Della-Giustina, Dr. Yi Luo and Dr. Christopher Mark for their assistance and advice throughout the course of this thesis. 


\section{Table of Contents}

$\begin{aligned} \text { Page } & \text { Pa }\end{aligned}$

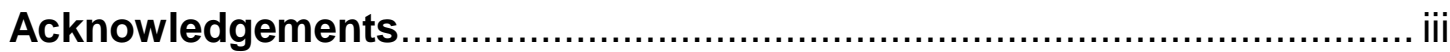

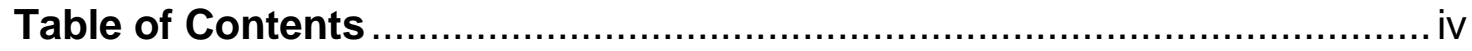

List of Tables ....................................................................................... vii

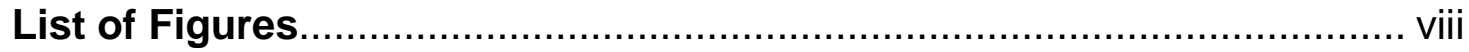

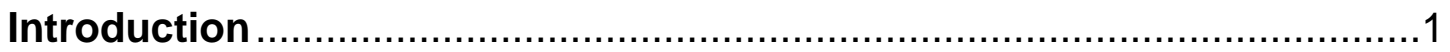

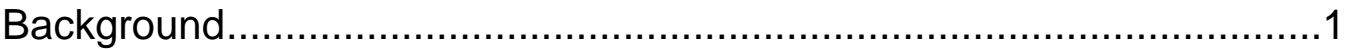

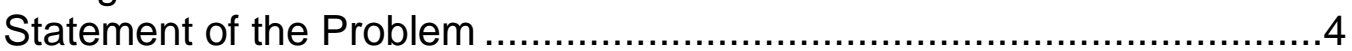

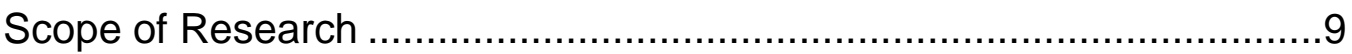

Organization of Thesis ............................................................

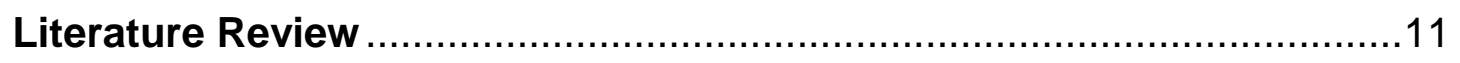

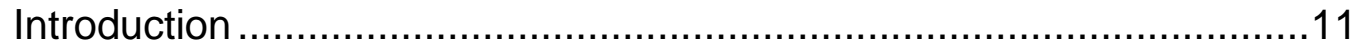

Review of Pillaring Methods...................................................... 12

Early Mechanized Pillar Mining ............................................... 12

Open-End Method ..................................................... 15

Pocket-and-Wing Method ............................................17

Modern Mechanized Pillar Mining .....................................18

Remote Controlled Continuous-Miner .................................18

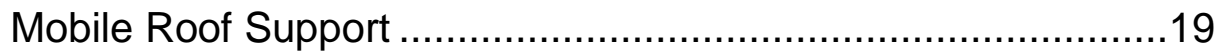

Pillaring Methods ......................................................22

Geotechnical Aspects of Pillaring Risk Factors.............................26

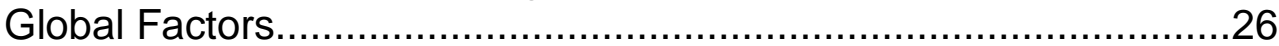

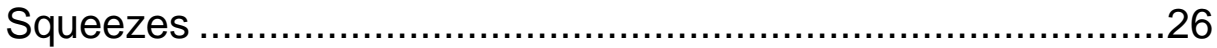

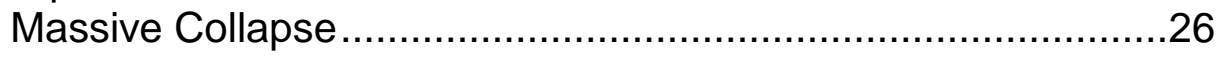

Pillar Bump ..............................................................27

Local Stability Factors .................................................... 27

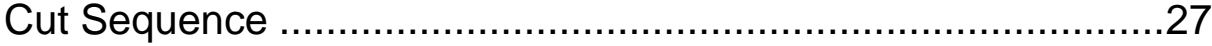

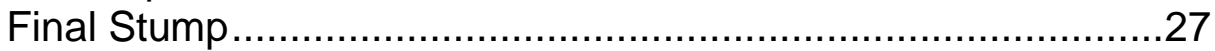




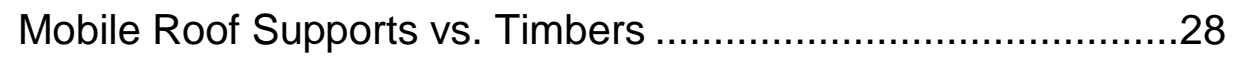

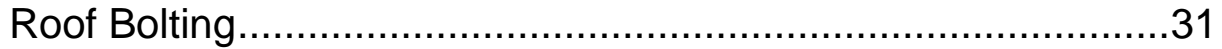

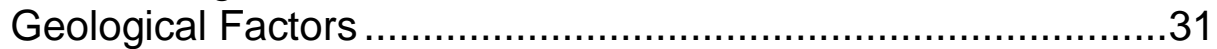

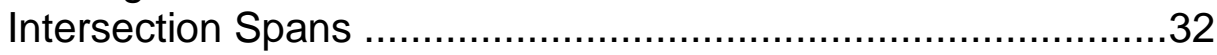

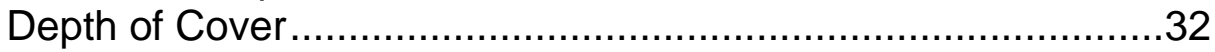

Multiple Seam Interactions................................................32

Recovery of Older or Non-Uniform Pillars ................................ 32

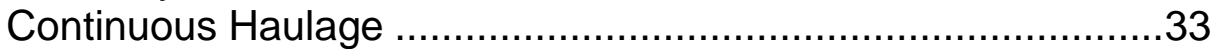

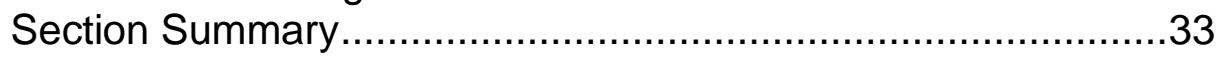

Mineworker Performance on Pillaring Sections ..................................35

Review of Statistics Related to Pillar Extraction .........................35

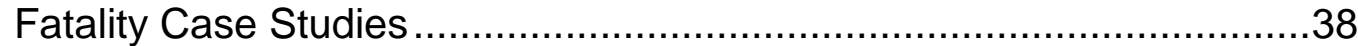

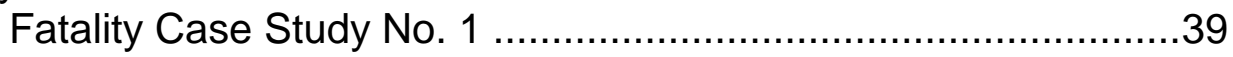

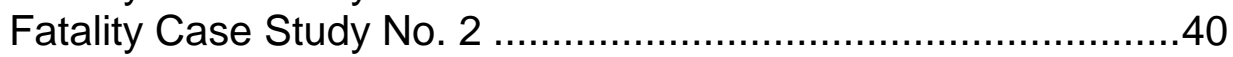

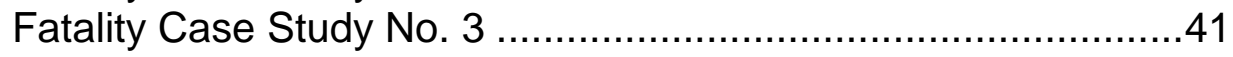

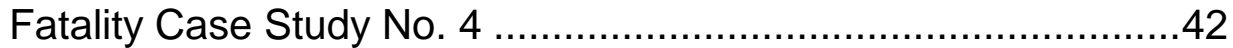

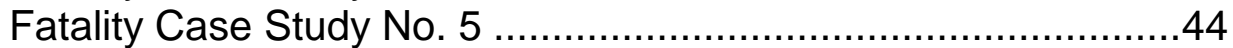

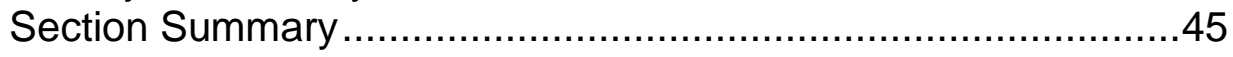

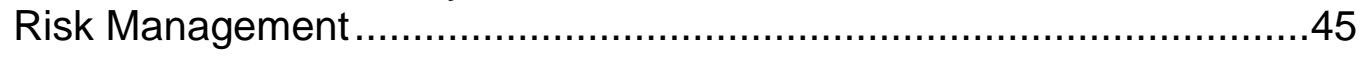

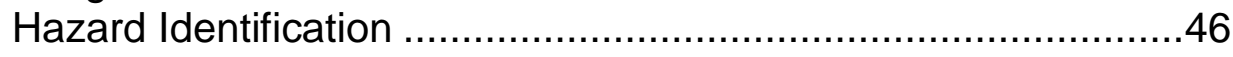

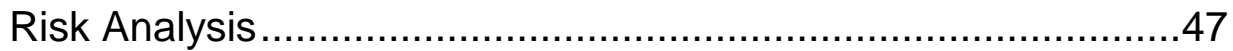

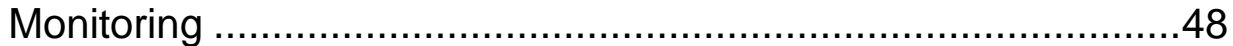

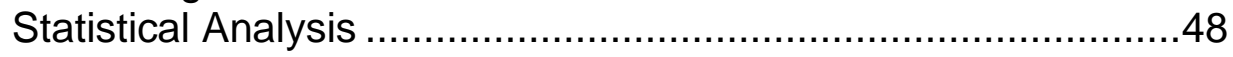

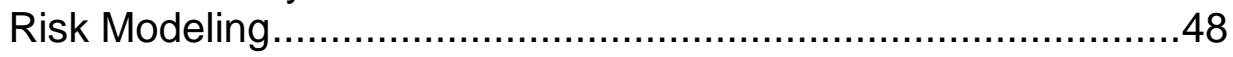

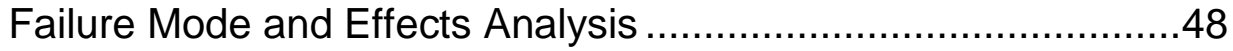

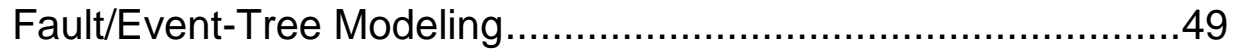

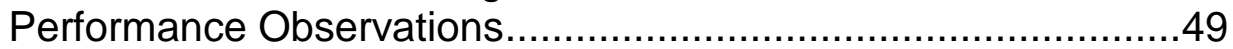

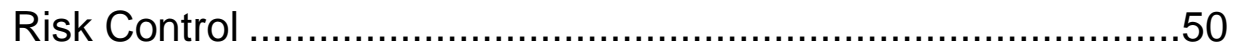

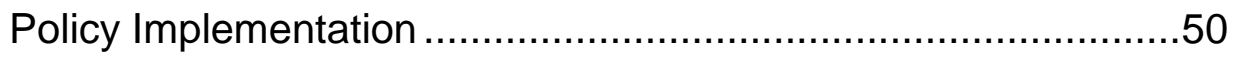

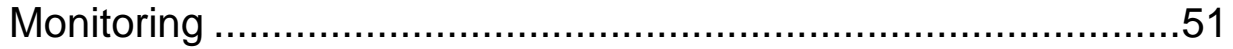

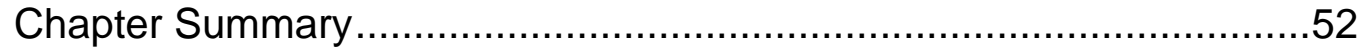

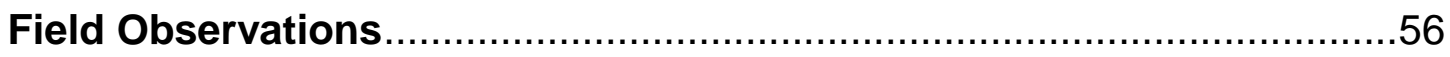

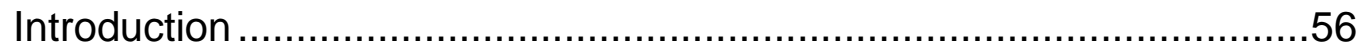

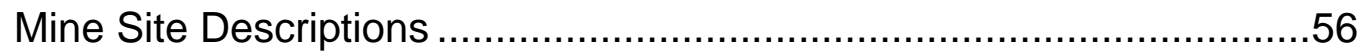

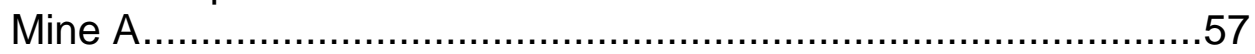

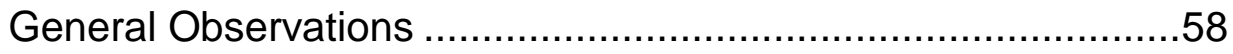

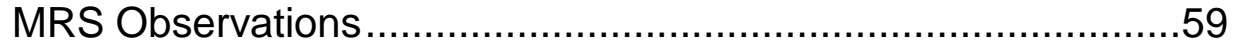

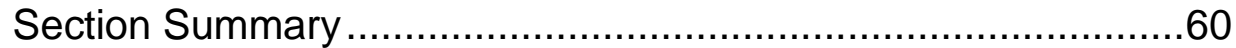

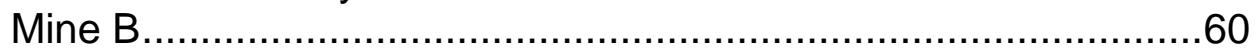

General Observations Visit No.1 ...........................................63

MRS Observations Visit No.1.............................................63

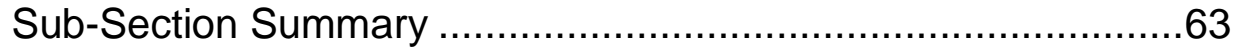

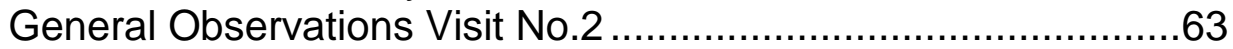




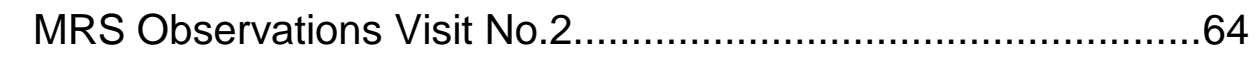

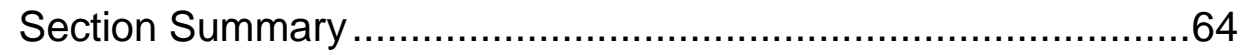

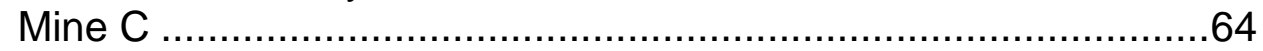

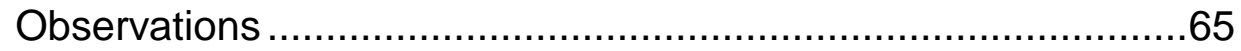

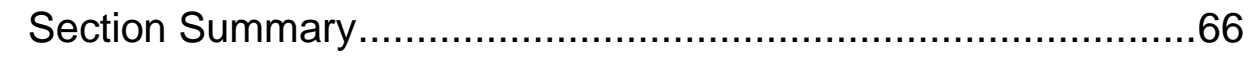

Job Description Observations ........................................................66

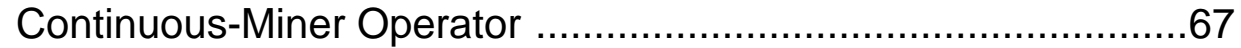

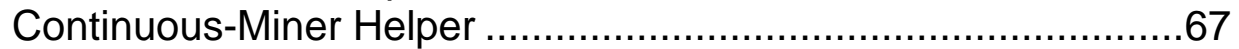

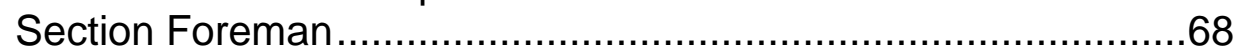

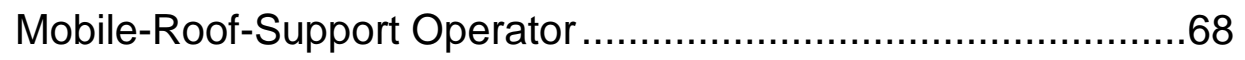

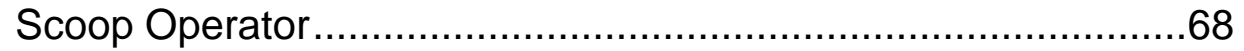

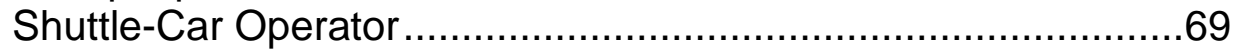

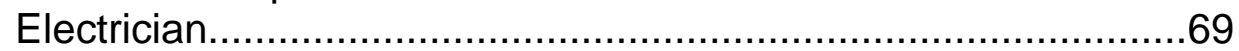

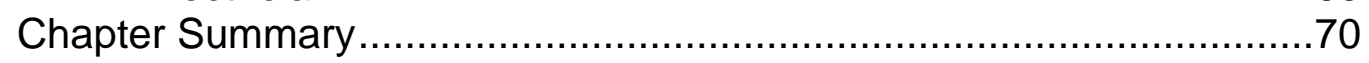

\section{The Development of a Workplace Sampling}

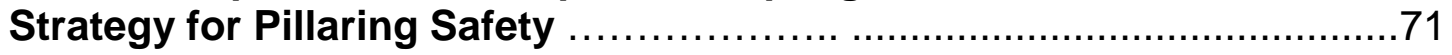

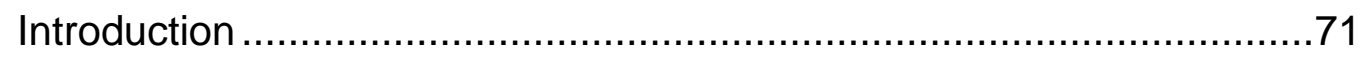

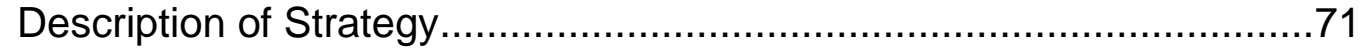

Engineering Risk Factors ...............................................

Personnel-Based Risk Factors................................................71

Development of Sampling Forms...................................................

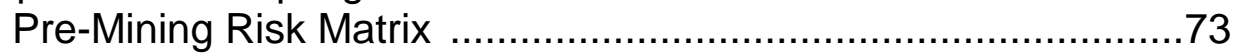

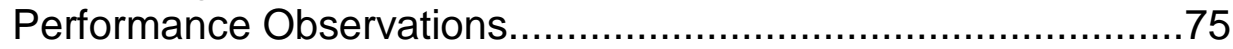

Unsafe Worker Performance Identification..............................75

Continuous-Miner Observation Form ...................................77

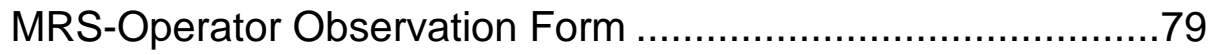

Mineworker-Location Observation Form ……......................... 81

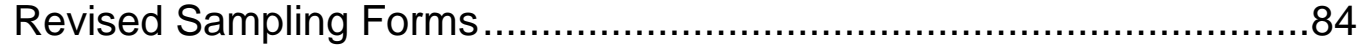

Pre-Mining Risk Matrix ...........................................................84

Unsafe Worker Performance Identification Form ......................86

Activity and Location Monitoring Form ....................................88

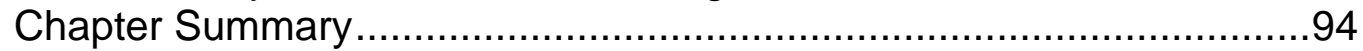

\section{Application of the Workplace Sampling Strategy}

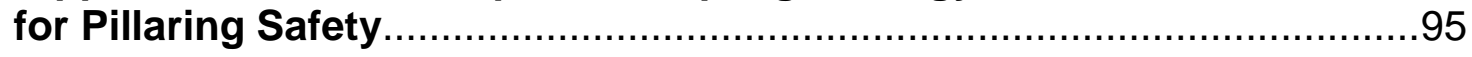

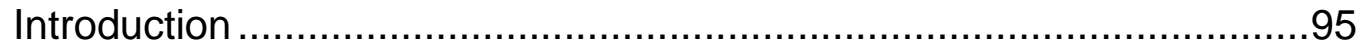

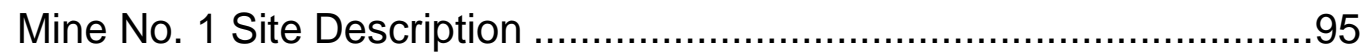

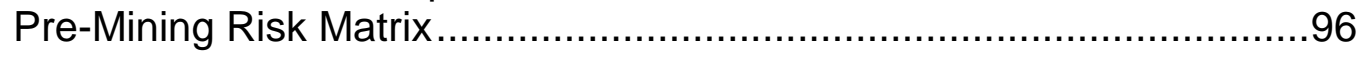

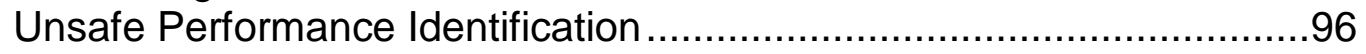

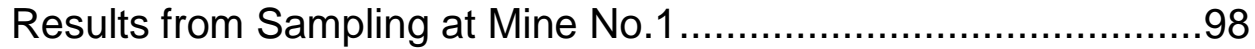

Activity and Location Monitoring ..................................................101 


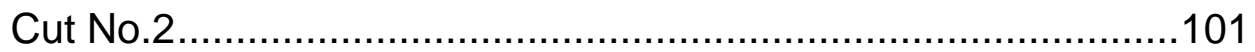

Exposure to the Active Mining Zone ....................................103

Activity Timing ..............................................................107

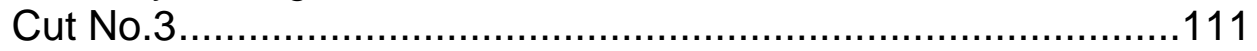

Exposure to the Active Mining Zone ...................................112

Activity Timing .............................................................116

Summary of Results ...........................................................119

Mine Site No. 2 Description and Summary ..................................120

Estimation of Time Requirements.............................................122

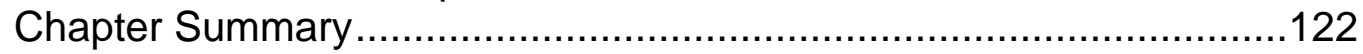

\section{Summary, Conclusions and Recommendations for}

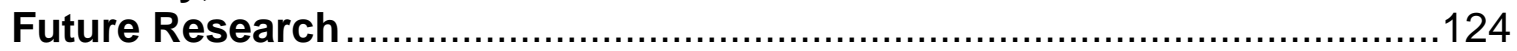

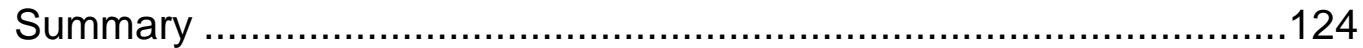

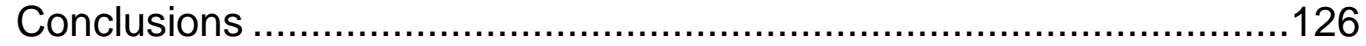

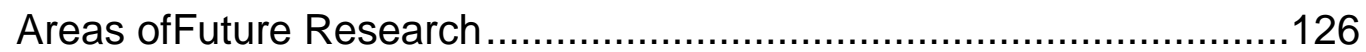

Improved Remote Control Systems ..........................................126

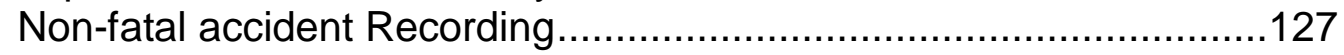

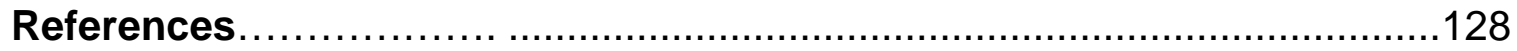

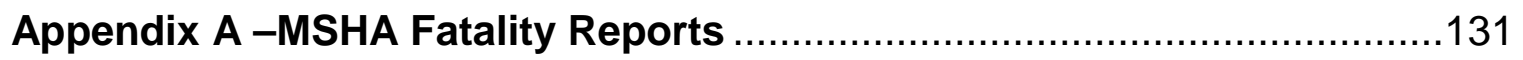

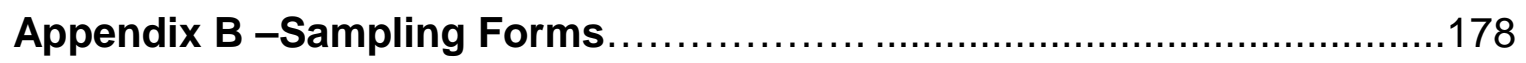

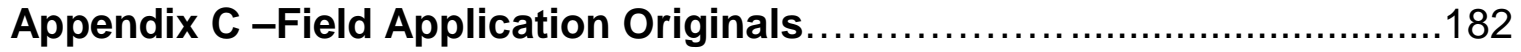




\section{List of Tables}

Page

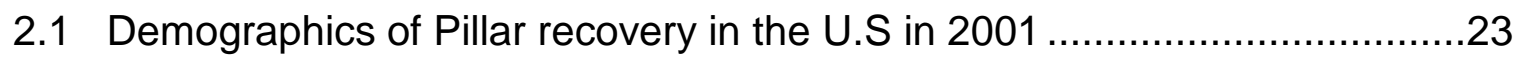

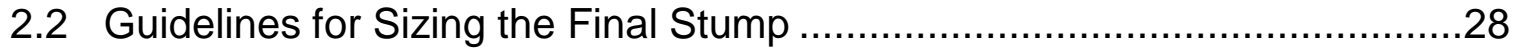

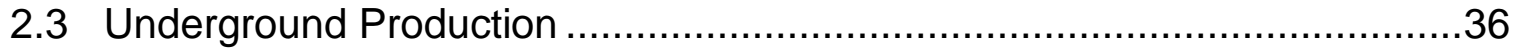

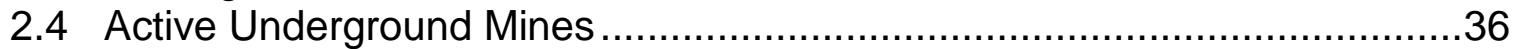

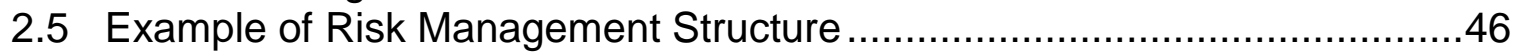

\section{List of Figures}

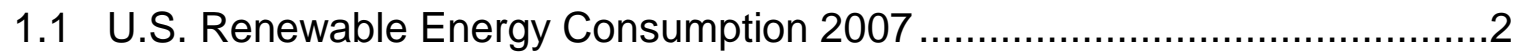

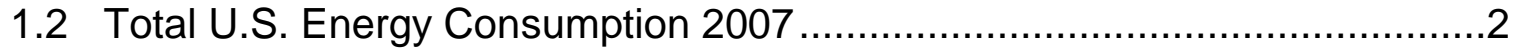

1.3 U.S. Fossil Fuel Energy Consumption 2007 ..........................................

1.4 U.S. Electrical Power Production by Fuel Source 2007 ............................4

1.5 U.S. Electrical Power Generation by Coal and Cost per kWh .....................5

1.6 Current U.S. Coal Reserves and Total Resources ..................................6

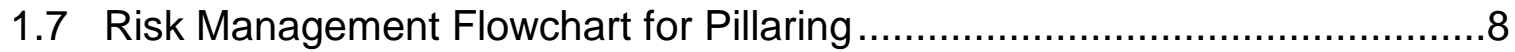

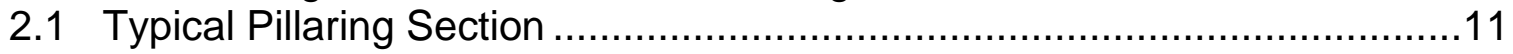

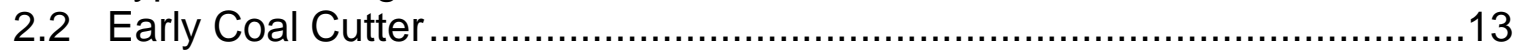

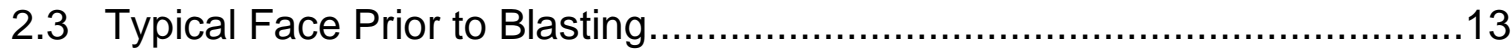

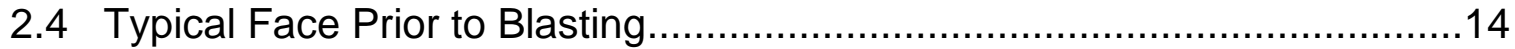

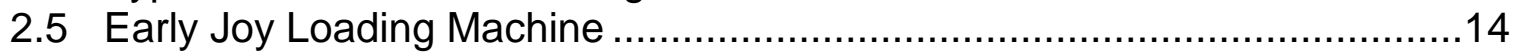

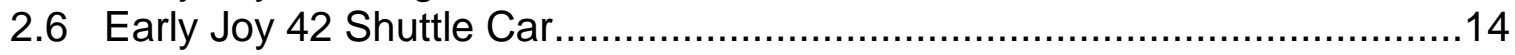

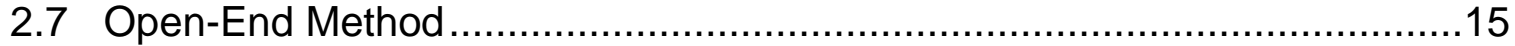

2.8 Example of Open-End Method of Pillar Extraction ..................................16

2.9 Example of Pocket-and-Wing Method of Pillar Extraction .......................17

2.10 Remote Controlled Continuous Miner ....................................................18

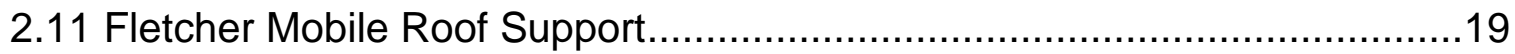

2.12 Example of Christmas-Tree Cut Sequence ........................................24

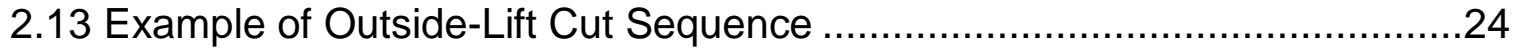

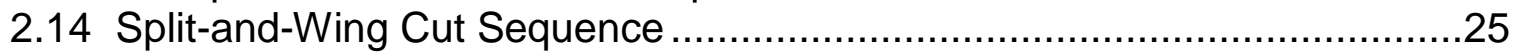

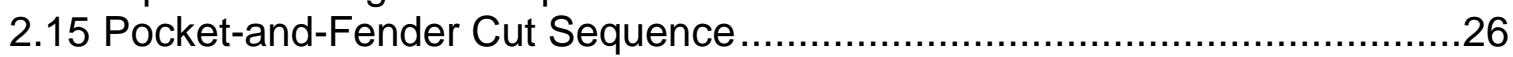

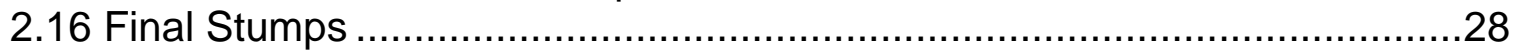

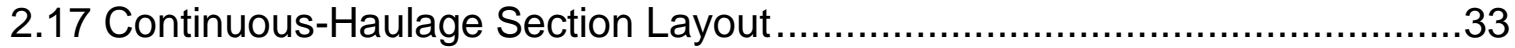

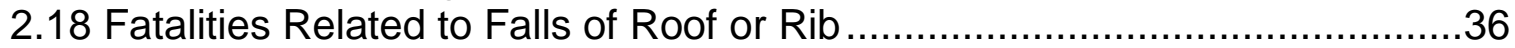

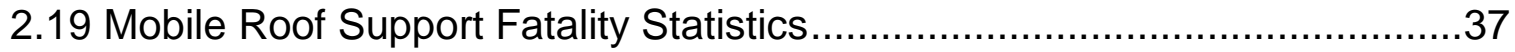

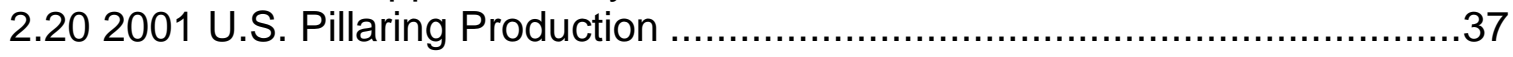

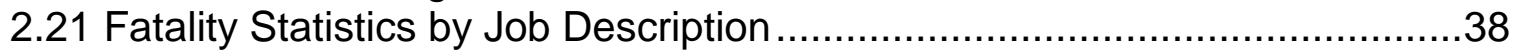

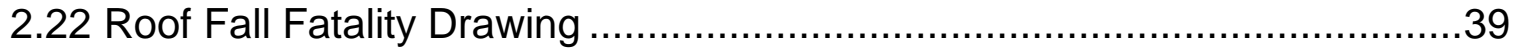

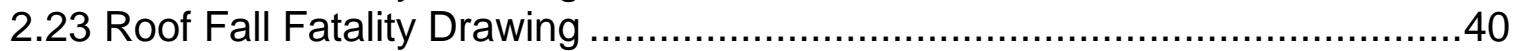




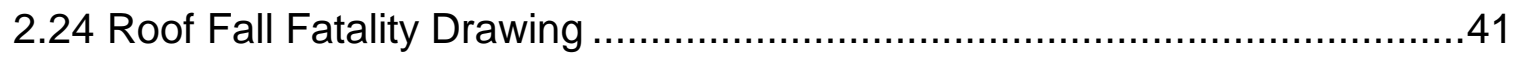

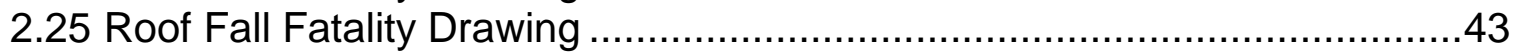

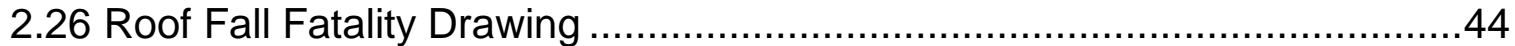

2.27 Risk Management Flowchart for Pillaring ..........................................54

3.1 Mine A Observed Dimensions and Roof-Bolting Pattern ..........................58

3.2 MSHA Approved Christmas-Tree Cut Sequence Utilized at Mine B ...........61

3.3 MSHA Approved Outside-Lift Cut Sequence Utilized at Mine B .................62

3.4 Mine C Observed Dimensions and Roof-Bolting Pattern..........................65

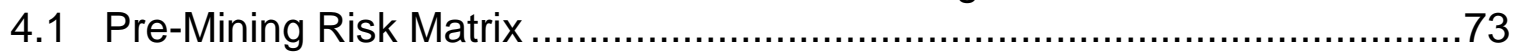

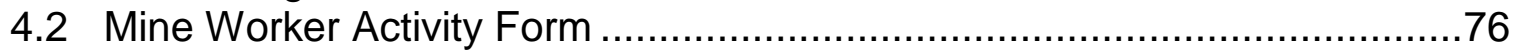

4.3 Example of the Continuous-Miner Observation Form .............................77

4.4 Example of the Zone Division for Continuous-Miner Observation ...............78

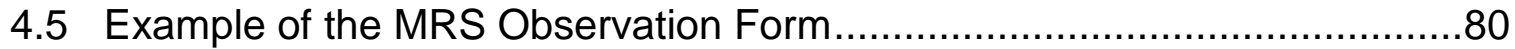

4.6 Example of the Zone Division for MRS Observation ...............................81

4.7 Example of the Observation Form for Mineworker Location.......................82

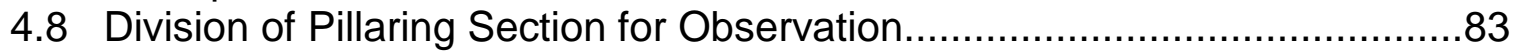

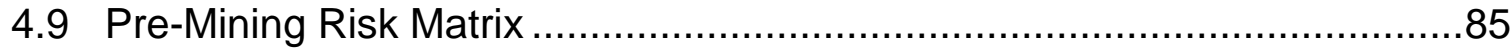

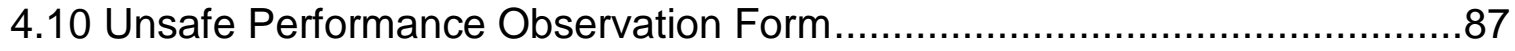

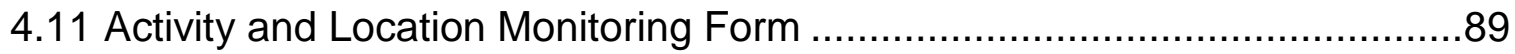

4.12 Example of Activity and Location Monitoring Form..................................90

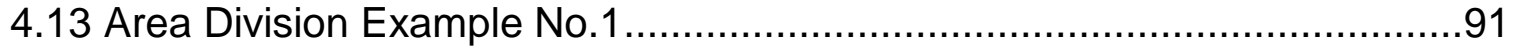

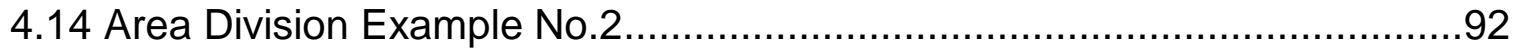

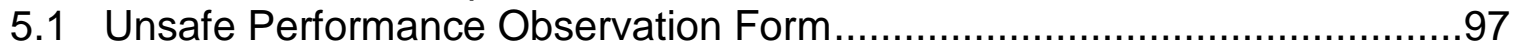

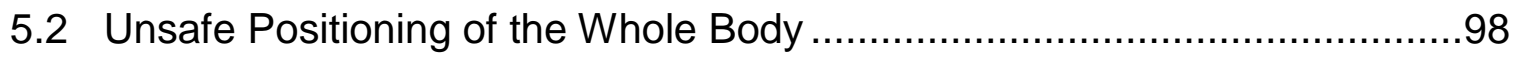

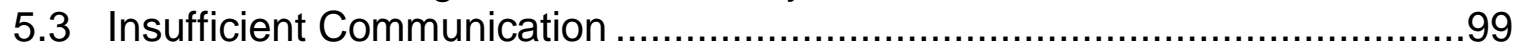

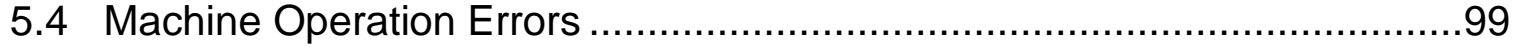

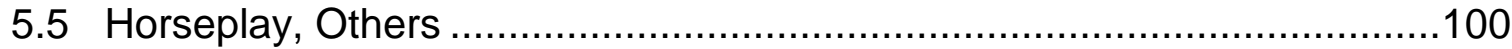

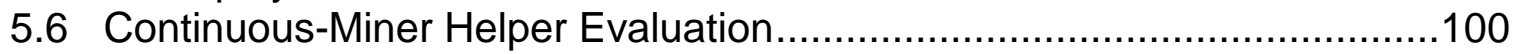

5.7 Area Division and Cut Numbering for Cut No.2 ..................................102

5.8 Continuous-Miner Operator Exposure .............................................104

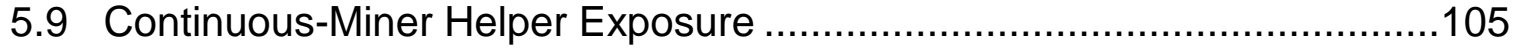

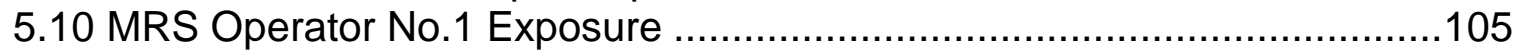

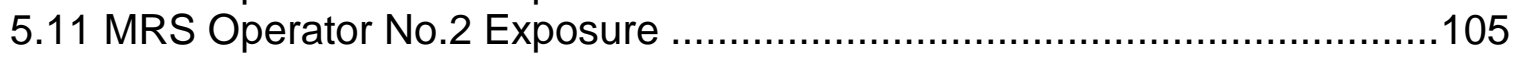

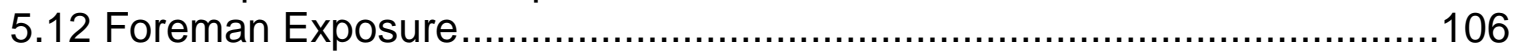

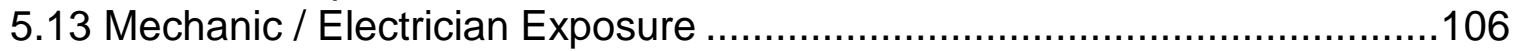

5.14 Exposure to the Active Mining Zone by Job Description ........................107

5.15 Activity Timing for the Continuous-Miner Operator .................................108

5.16 Activity Timing for the Continuous-Miner Helper ....................................108

5.17 Activity Timing for the MRS Operator No.1 …...................................109

5.18 Activity Timing for the MRS Operator No.2 …..................................109

5.19 Activity Timing for the Foreman......................................................

5.20 Activity Timing for the Mechanic / Electrician .......................................110

5.21 Area Division and Cut Numbering for Cut No.3 .....................................111

5.22 Continuous-Miner Operator Exposure .............................................112

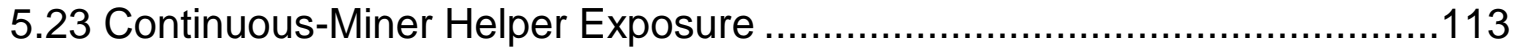

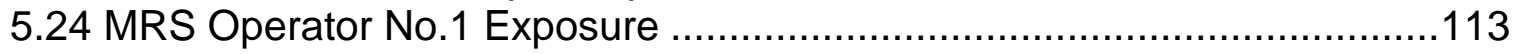




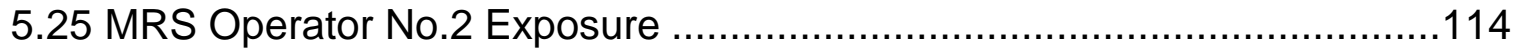

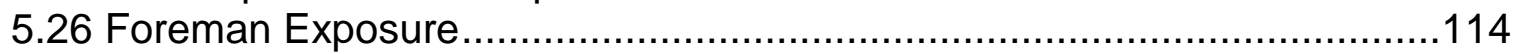

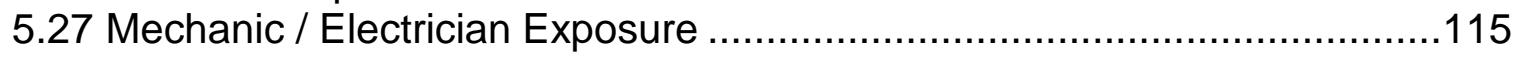

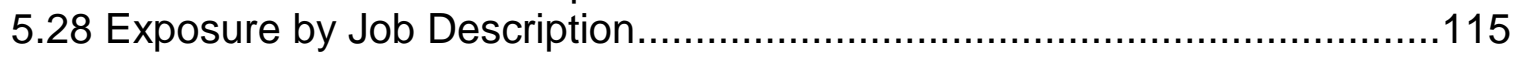

5.29 Activity Timing for Continuous-Miner Operator.....................................116

5.30 Activity Timing for Continuous-Miner Helper .......................................117

5.31 Activity Timing for MRS Operator No.1 ….......................................117

5.32 Activity Timing for MRS Operator No.2 …...................................118

5.33 Activity Timing for Foreman...........................................................118

5.34 Activity Timing for Mechanic / Electrician ..........................................119

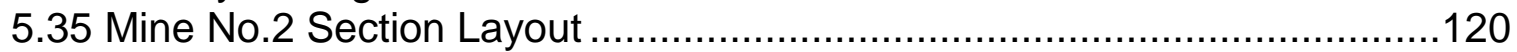

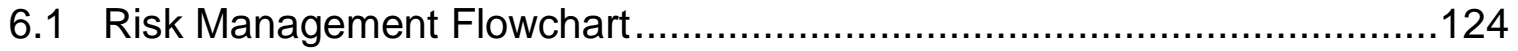




\section{CHAPTER 1. INTRODUCTION}

\section{Background}

Over the past 35 years, the United States has lacked a cohesive energy plan that utilizes domestic sources of energy to their fullest potential and, as a consequence, the nation continues to struggle in meeting its ever-expanding energy needs. This country cannot function without reliable energy supplies. By importing energy, the United States is at the mercy of foreign nations to maintain a dependable, fairly priced supply. Due to the ever-changing global political climate, the U.S. cannot be content to allow foreign nations to adversely influence the nation through embargos or price gouging.

The United States has been blessed with an abundance of natural resources, and the technological capabilities to make the nation energy independent. So why are we not expanding our domestic forms of energy, instead transferring billions of dollars from our economy to those of foreign countries? The United States does have numerous options when it comes to energy production. These traditional and nontraditional options include, but are not limited to, the following: petroleum, natural gas, coal, nuclear, hydro-power, geothermal, bioenergy, solar, and wind. As can be seen in Figure 1.1, energy sources such as solar and wind, that have been touted as energy curealls by environmental activists, make up only a small portion of the renewable energy market and are mostly irrelevant in terms of national energy usage, accounting for less than $0.5 \%$ of overall energy consumption (Figure 1.2). The vast majority of U.S. energy usage comes from fossil fuels, and this trend should continue for decades before nuclear and renewable sources of energy are capable of significantly altering the mix, let alone meeting increased short-term demand. Therefore, it is obvious that the short-term increased energy demand can only be satisfied with expansion or increased recovery from existing operations of fossil fuels. 


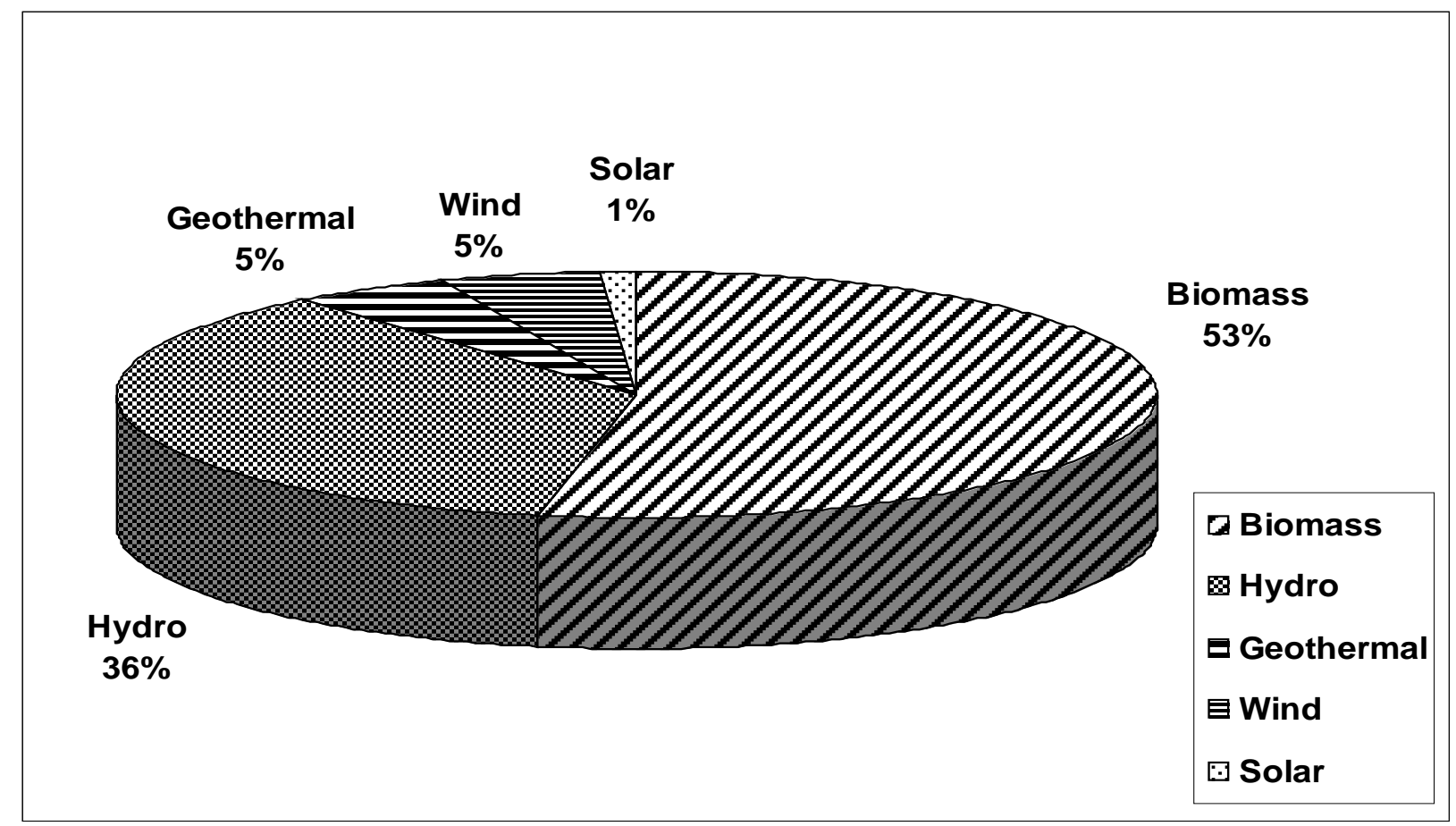

Figure 1.1. U.S. Renewable Energy Consumption 2007 (United States Energy Information Administration, 2008)

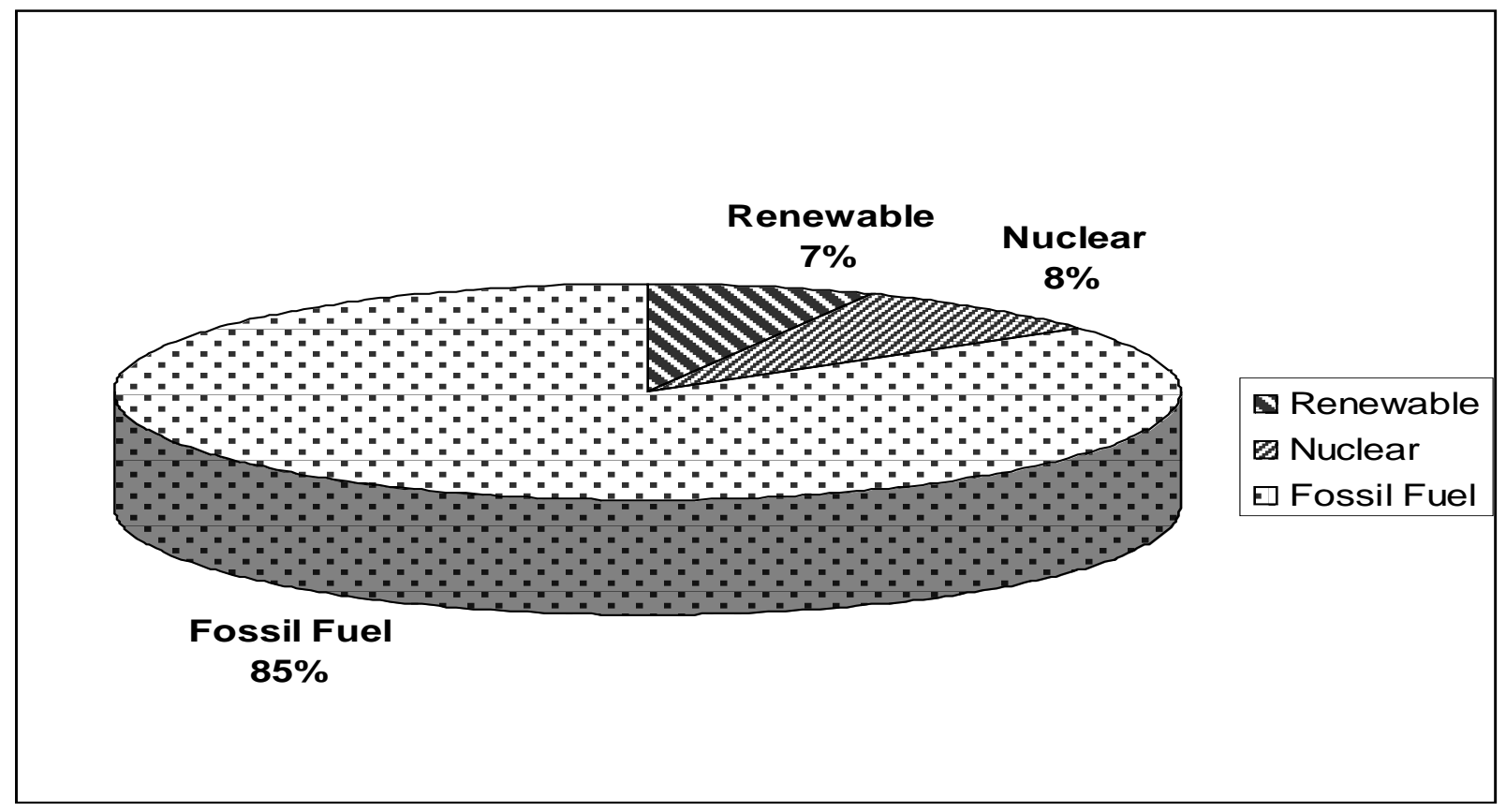

Figure 1.2. Total U.S. Energy Consumption 2007 (United States Energy Information Administration, 2008)

Fossil fuels account for approximately $85 \%$ of the total energy consumed in the United States, and over two-thirds of the electrical power generation. Fossil fuels 
include: petroleum, natural gas, and coal (Figure 1.3). When overall energy consumption is considered, petroleum is the dominant fuel source. However, the overall energy usage includes the energy expended by automobiles and other transportation systems that burn petroleum-based fuels. This consumption as a transportation fuel is what drives petroleum far above coal and natural gas in terms of overall energy consumption.

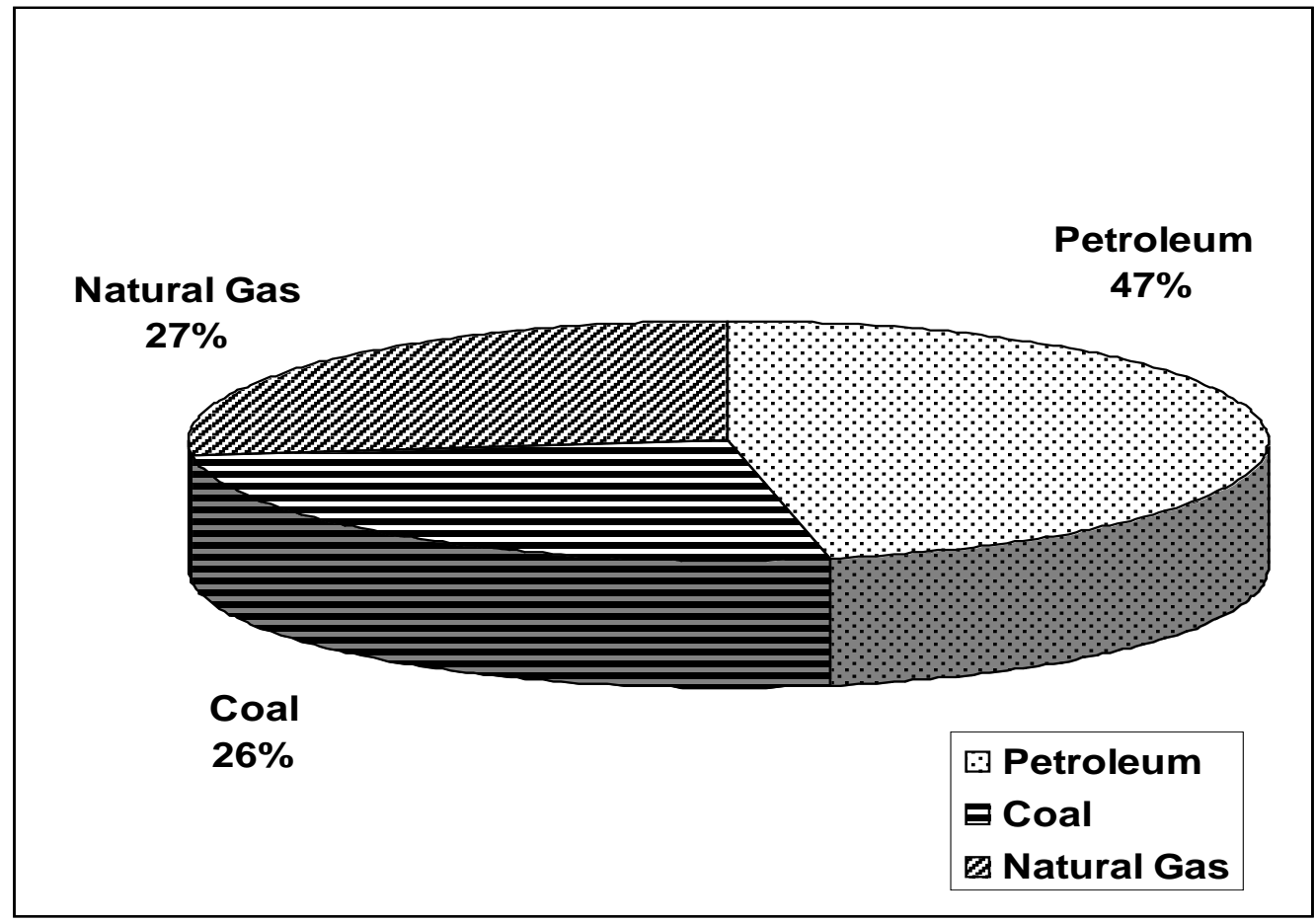

Figure 1.3. U.S. Fossil Fuel Energy Consumption 2007 (United States Energy Information Administration, 2008)

Even though, in terms of overall energy consumption, petroleum is the dominant fuel, coal reigns supreme in terms of electricity production. Coal accounts for approximately $50 \%$ of the electricity generated in the U.S., a percentage approximately twice that of its closest competitors, nuclear and natural gas (Figure 1.4). Other sources of electrical energy have not even approached the magnitude of coal. Coal-fired power plants provide some of the lowest-cost electricity in the nation (Figure 1.5) and are crucial for the nation's economic success. Beyond coal's role as a primary electrical producer, coal is also a crucial product for the steel industry, which in turn affects the construction and automotive industry and many other parts of our economy. What makes coal so precious is not only its many usages and its impact on our economy, but also its ready domestic availability. 


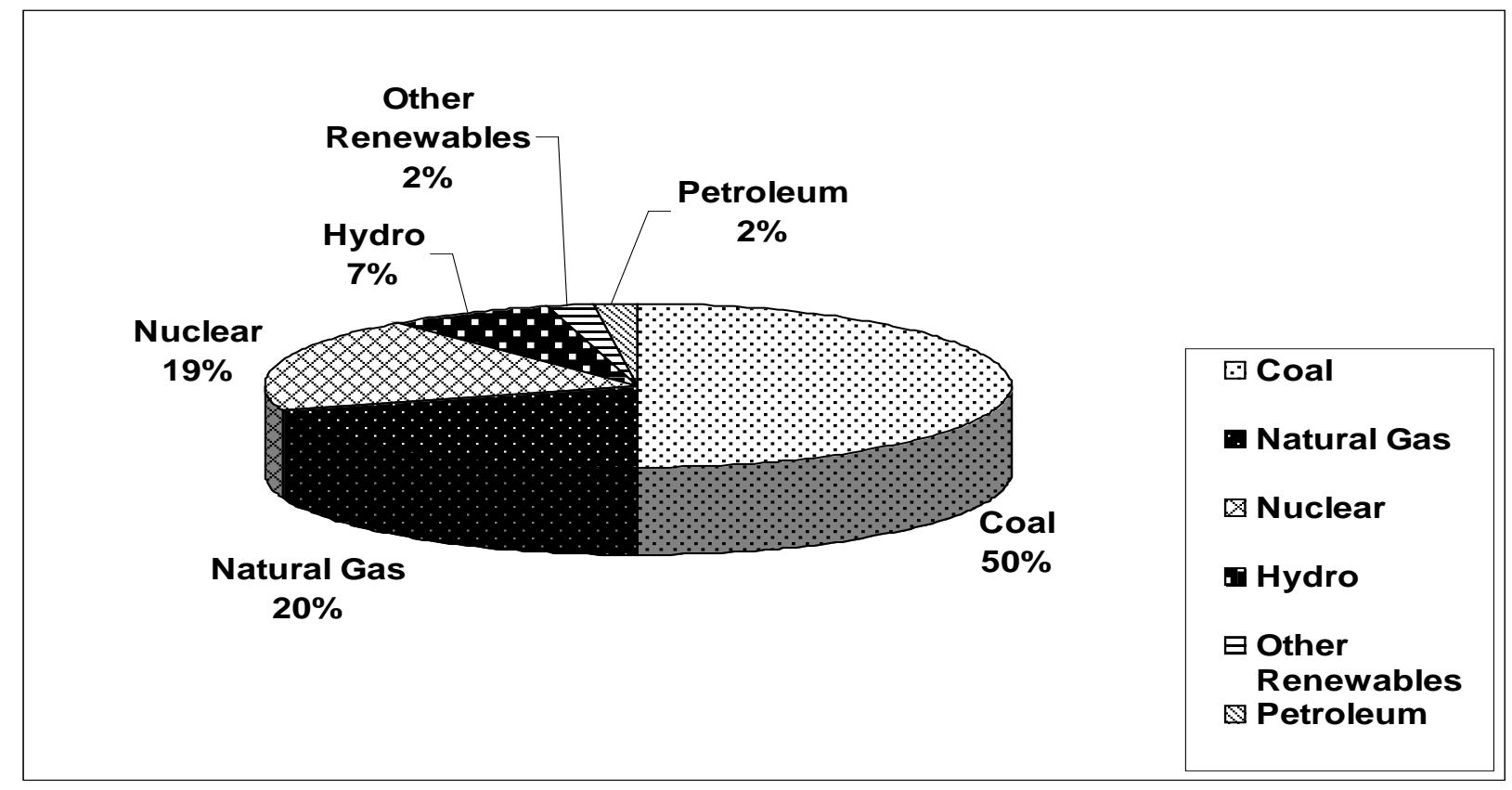

Figure 1.4. U.S. Electrical Power Production by Fuel Source 2007 (United States Energy Information Administration, 2008)

Coal is the most plentiful of the fossil fuels in the United States, with domestic reserves larger than petroleum and natural gas reserves combined (USDOE, 2008). In 2007, the United States produced 1.146 billion tons of coal, and operated 1,374 mines. Of the 1.146 billion tons of coal produced, 351.79 million tons were produced by underground mining, while the remaining 793.69 million tons were produced by surface mining. Currently, the coal mining industry employs approximately 100,000 miners (MSHA, 2008) and, in many areas, represents the foundation for regional economies. Obviously, whether producing steam-grade coal for electricity generation or metallurgical-grade coal for use in steel production, coal mining is a necessary industry and an asset to our nation. 


\section{Coal Helps to Produce Low-Cost Electricity \& a Better Quality of Life}

\section{Cost Per kWh \& Percent of Coal Generation}

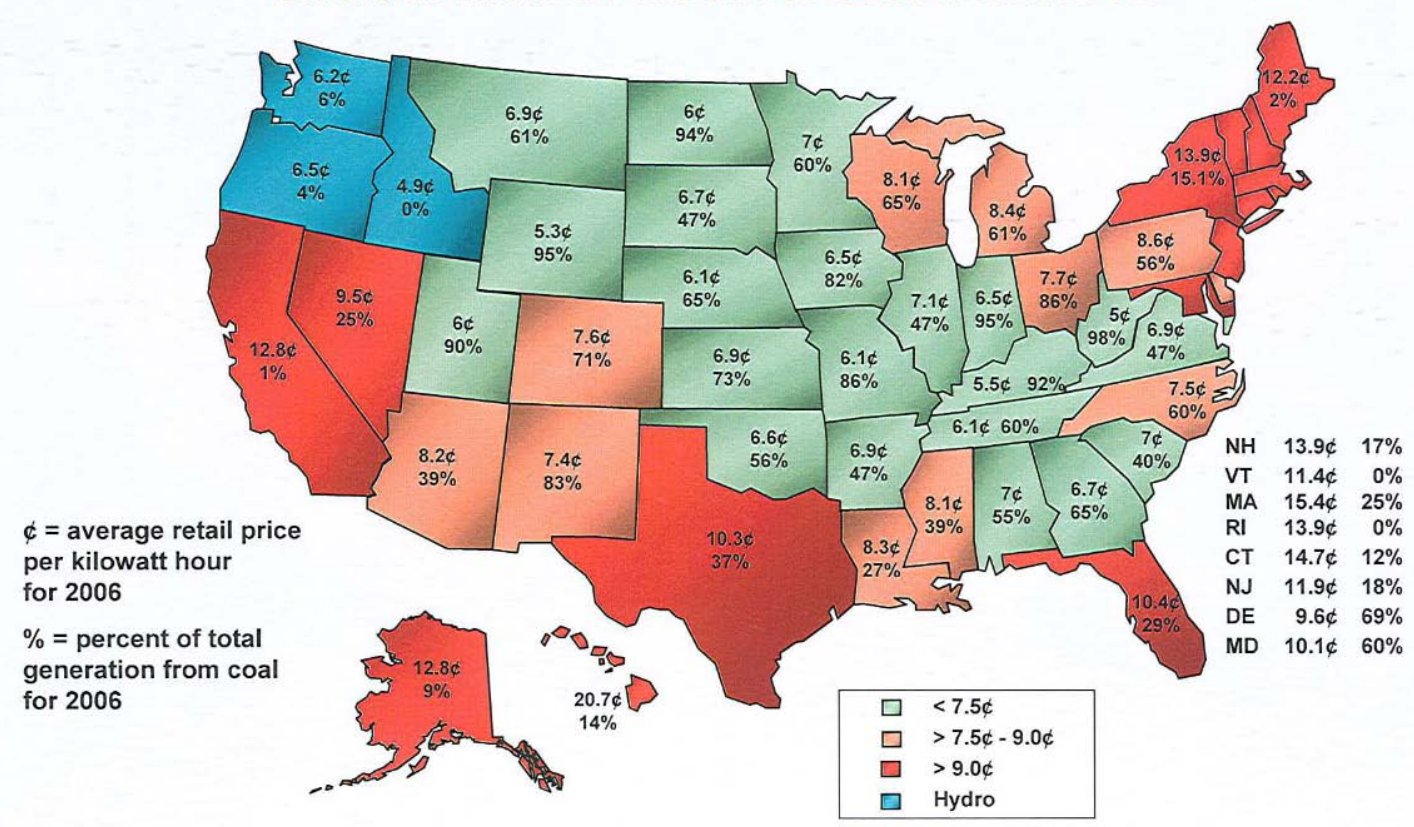

Source: Energy Information Administration, March 2007

Figure 1.5. U.S. Electrical Power Generation by Coal and Cost per kWh, (United States Energy Information Administration, 2007).

As previously stated, coal reserves dwarf those of petroleum and natural gas; however, a large portion of the most favorable reserves in terms of extraction have been mined, leaving deeper and more discontinuous coalbeds to challenge long-term future production. If coal is to contribute to the increased energy needs of the United States, more tonnage will have to come from existing operations and coalbeds that are considered mineable by today's standards. This necessity for higher tonnages requires a higher percent extraction of the coalbeds to ensure that this natural resource is used most efficiently, and not wasted.

Although the United States has over 200 years worth of potential coal output at current levels of production, technology, and economics, only a small portion is considered recoverable from existing mines. For short-term production gains, however, it will be necessary to increase production and recovery from existing operations (Figure 1.6). How can production and recovery be increased from existing mines? One possible answer would be to expand pillar recovery. Unfortunately, because of the risk related to pillar recovery, many operations forgo the added recovery, even when geotechnical and geometric mining conditions are favorable. But the current economic climate demands that continued evaluation should be provided to coal-pillar recovery. Pillar extraction is one approach that can enable coal to contribute to increased energy production, if the 
issues of the safety hazards inherent with coal-pillar extraction can be satisfactorily addressed.

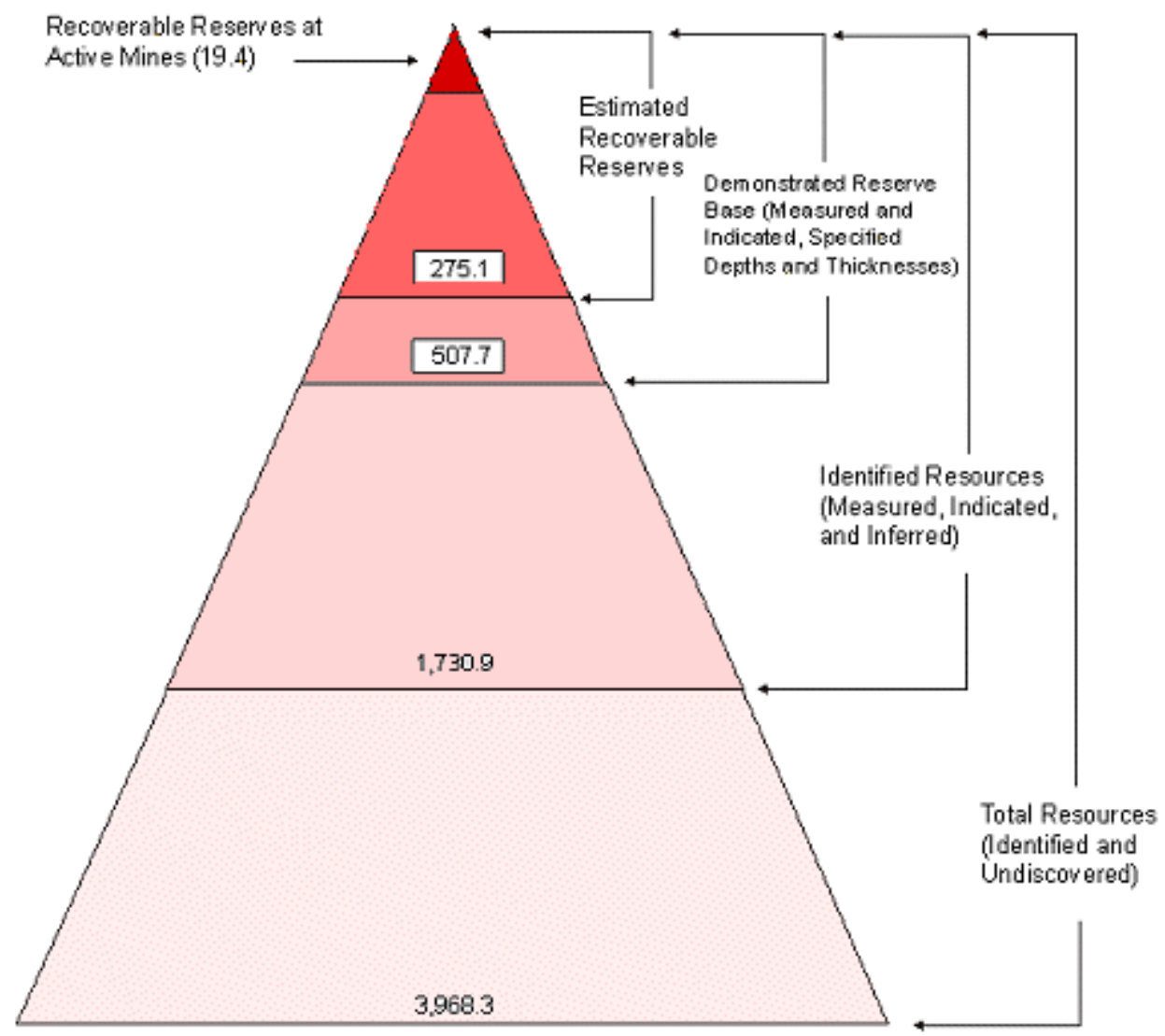

Figure1.6. Current U.S. Coal Reserves and Total Resources in billions of tons (United States Energy Information Administration, 2003).

\section{Statement of the Problem}

The high current and forecasted demand for coal warrants consideration of techniques designed to increase production and recovery of our nation's coal resources from existing operations. Underground pillar extraction is one such technique, but it is frequently overlooked because of the additional risks involved with deliberate caving of the immediate roof in the mining section.

Pillaring accounts for approximately $10 \%$ of the annual coal production in the United States (Mark, et al., 2003) and, as the demand for coal rises, it is reasonable to believe that pillaring will remain an essential part of coal-mine production; the problem is to safely increase that percentage. Between 1995 and 2007, pillaring has accounted for $38 \%$ of roof and rib fall fatalities (MSHA, 2008). The dangers associated with this type of mining underscores the debate as to whether the extra risk to the safety of miners is 
worth the additional coal that will be recovered. Although there are very strong opinions both in favor and in opposition to pillaring, it would be difficult to replace the increased production and recovery percentage from this form of production. The problem is how to better manage the risks of this technique in order to profit from its obvious positive attributes.

There are many risk factors associated with pillaring. Past research (Mark, et al., 2003) on pillaring has identified numerous risk sources, such as the following:

- Depth of cover

- Pillar sizing

- Roof support

- Cut sequence

- Geology

- Intersection span

- Multiple seam interaction

- Age and uniformity of pillars

- Continuous haulage, as it affects the design of entries and crosscuts.

In the case of pillaring, the risks are high, and poorly made decisions to move forward under undesirable mining conditions can prove very costly both in a monetary sense as well as the cost of human life. However, a close inspection of this list indicates that the risks have been attributed, almost exclusively, to geotechnical considerations. There has been a lack of emphasis on the other side of the equation that affects overall risk: how effectively the mineworkers perform their assignments on a pillaring section. In other words, a coalbed could be judged to be low risk for pillaring, for an appropriate mining technique, but may have its risk intensified by deficient mineworker performance in the mining process. Figure 1.7 indicates the manner in which risk management should actively minimize risk from both geotechnical and mineworker-performance perspectives. When planning a mining section, engineers and other management personnel have to determine whether or not the possible gains outweigh the risks associated with the extraction technique. By applying the principles of risk management to pillaring, both in the planning and operational stages, it may be possible to reduce the overall risk associated with pillaring and make the technique more desirable. 


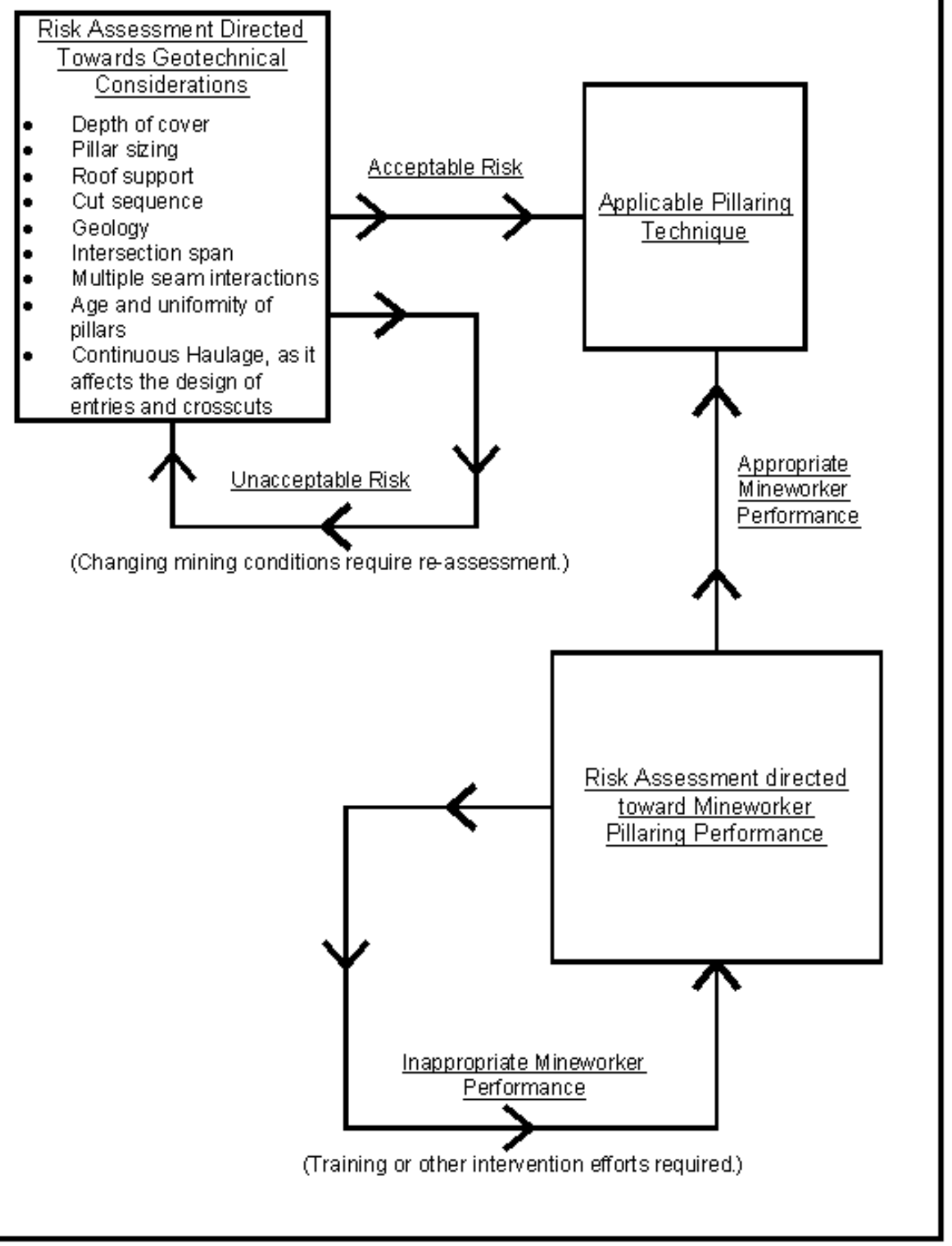

Figure 1.7. Risk Management Flowchart for Pillaring (Bise, 2007) 


\section{Scope of Research}

This thesis will approach the topic of increasing U.S. coal production and recovery through a more structured approach to minimizing the risks of pillar extraction. Most high-risk hazards, such as those previously listed, have been addressed through previous geotechnical research, but the technique of pillar extraction still remains as a high-risk activity. Therefore, the strategy that will be followed in this thesis is to evaluate established risk-control approaches by today's standards, implement newly developed approaches, and then address those lesser, but ever-present, risks which limit the number of successful pillaring operations. This thesis will also build on the foundations of risk management established by previous research as well as incorporate successful risk management models from other industries with regard to human performance.

This thesis will present data from a combination of previously documented accident reports from MSHA, NIOSH, and the USBM, as well as observations from mine visits. The purpose of the mine visits is to provide first-hand experience on how these risks are being controlled, so that communication can be established with minemanagement personnel to identify hazards that are not being adequately controlled. Southern West Virginia has the highest concentration of pillaring operations of any state associated with coal mining, and accounts for nearly $46 \%$ of pillaring fatalities during the past ten years. The Central Appalachian coalfields of southern West Virginia also contain all of the previously discussed geotechnical and mineworker performance based risk sources. As a result, the mine visits will take place primarily in the southern West Virginia region.

\section{Organization of the Thesis}

This thesis on the development of a risk management approach to pillar extraction in the Central Appalachian coalfields will be organized in the following manner:

- Chapter 2 will contain a Literature Review of pillaring risk factors, pillaring fatality statistics and risk management methodology.

- Chapter 3 will provide case-study information gathered from field trips to various representative mines in West Virginia, as well as a review of fatality case studies related to pillaring.

- Chapter 4 will propose a Risk Management strategy and will detail the observation methodology used to assess the performance of miners on a pillaring section.

- Chapter 5 will be a field evaluation of the performance evaluation strategy. The chapter will contain an actual study conducted at a mine extracting pillars in Central Appalachia, along with sample graphical representations of information extracted from the completed observation forms. This should serve as a template 
for performing this type of performance-based risk assessment at other mines.

- Finally, Chapter 6 will summarize the research conducted during this study, and will provide conclusions based upon the results achieved. A section on areas of future research will describe potential directions in which this study can be further developed. 


\section{CHAPTER 2. LITERATURE REVIEW}

\section{Introduction}

As a coal mine is developed, entries and crosscuts are driven into the coal seam. These entries and crosscuts result in coal pillars that are left in place to support the overburden (Figure 2.1).

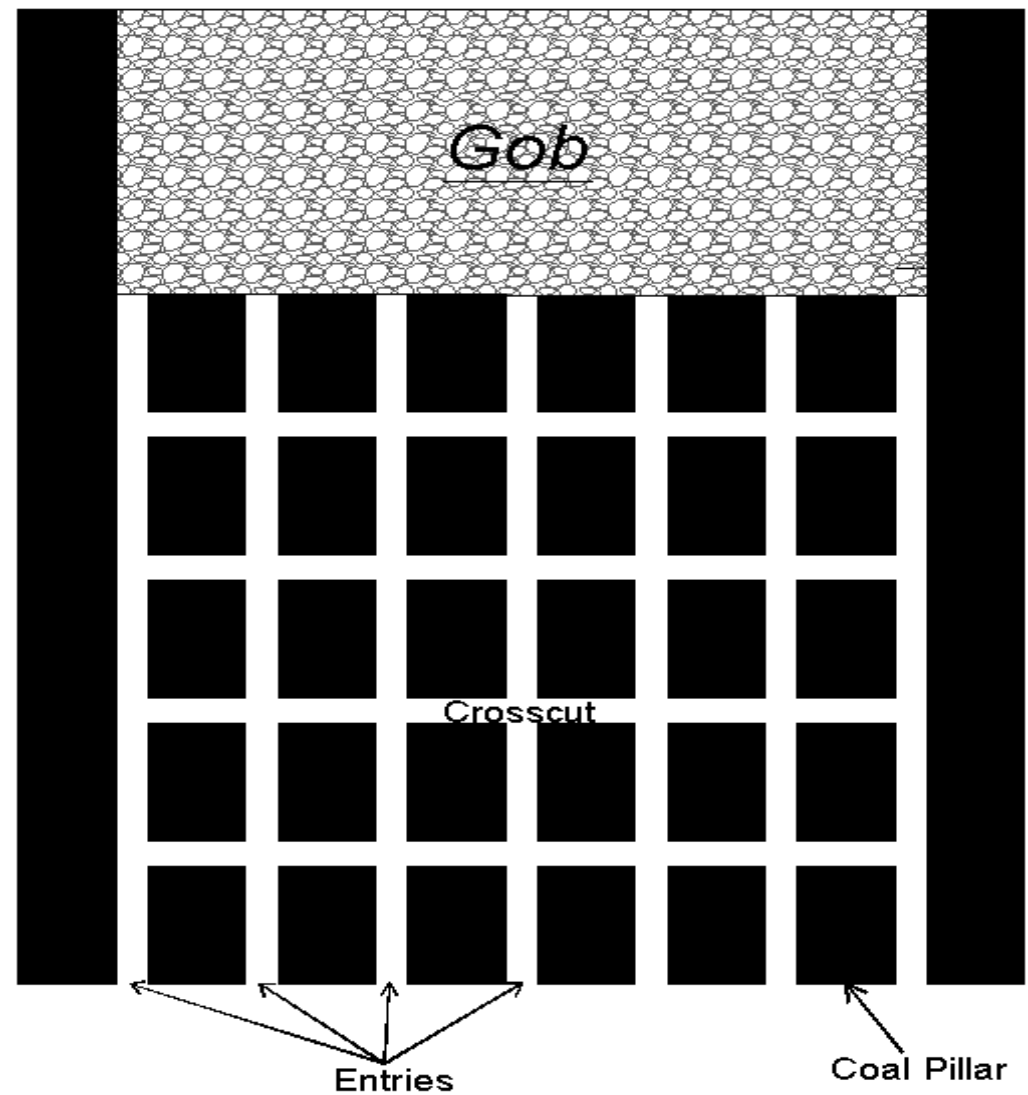

Figure 2.1. Typical Pillaring Section

Pillaring, also known as retreat mining or second mining, is the systematic extraction of these coal pillars when the mining unit is withdrawing from that portion of the mine. Pillaring provides for a higher extraction percentage of the coal seam as opposed to leaving the pillars in place. With the increasing demand for energy, achieving a higher extraction percentage of coal is desirable. Pillaring utilizes a continuous miner, mobile or fixed roof supports, and various haulage methods to extract coal, and has an advantage over longwall mining in that it requires a much lower capital investment and is capable of working in smaller, more discontinuous reserves. These traits make pillaring the ideal solution for achieving cost-effective high extraction in the Central Appalachian coalfields.

This chapter will discuss the evolution of pillar extraction in Central Appalachia, from early pillaring by conventional mining methods to today's modern mechanized 
pillaring. The chapter will also address risk factors associated with pillar extraction, as well as provide a review of pillaring-related statistics. Due to the fact that the focus of this research is on the practice of pillar extraction from a risk-management perspective, this chapter will conclude with a discussion on risk-management techniques and their possible application to pillaring.

\section{Review of Pillaring Methods}

As long as there has been coal mining in Central Appalachia, there has been pillaring. Early pillaring consisted of pick mining and hand loading. As technology and machinery advanced, these crude pick-and-shovel extraction techniques began to integrate modern technology. Initially, the mechanization of pillaring came with the utilization of coal cutters, explosives, and mechanized loaders. The next great evolution in pillaring closely followed the evolution of the continuous miner throughout its stages of development from chain-type, boring-type and drum-type cutter heads to the modern remote-controlled continuous mining machines that are utilized today. The most recent development that has drastically changed pillaring is the MRS (mobile roof support), developed to stabilize the working area and reduce the need for timber setting. It is the combination of remote-controlled continuous miners, MRS units, and modern haulage vehicles that represent the modern pillaring section.

\section{Early Mechanized Pillar Mining}

Since the days of hand loading are long gone, this section will focus on early mechanized pillaring by conventional mining methods and the evolution from conventional pillar mining methods to today's modern pillaring methods.

The major difference between conventional mining methods and modern methods is the manner in which coal is cut and loaded. Although technology has improved, the fundamental methods of coal haulage and roof bolting have remained basically the same. According to Stefanko (1983), conventional mining consists of the following steps:

1. Cutting,

2. Drilling,

3. Blasting,

4. Loading and hauling,

5. Roof bolting and the advancement of auxiliary services.

In mechanized conventional mining, the coal was cut using a coal cutter that resembles a giant chainsaw. The coal cutter was equipped with a cutter bar, usually 9 to $11 \mathrm{ft}$ long, with bits capable of cutting a 6-in slot or "kerf" at the bottom of the seam (Stefanko, 1983). While, in some instances, coal cutters were track mounted, most were rubber tired to provide better mobility (Figure 2.2). The process required the coal cutter to be moved to the face, and then it was used to make one and in some cases two horizontal cuts in the coal face to prepare it for blasting. The cutting of a coal seam prior to blasting is necessary because the kerf left by the cutting process permits the expansion of the coal during the blast, thereby requiring much less explosives than if the coal was shot from the solid (Stefanko, 1983). 


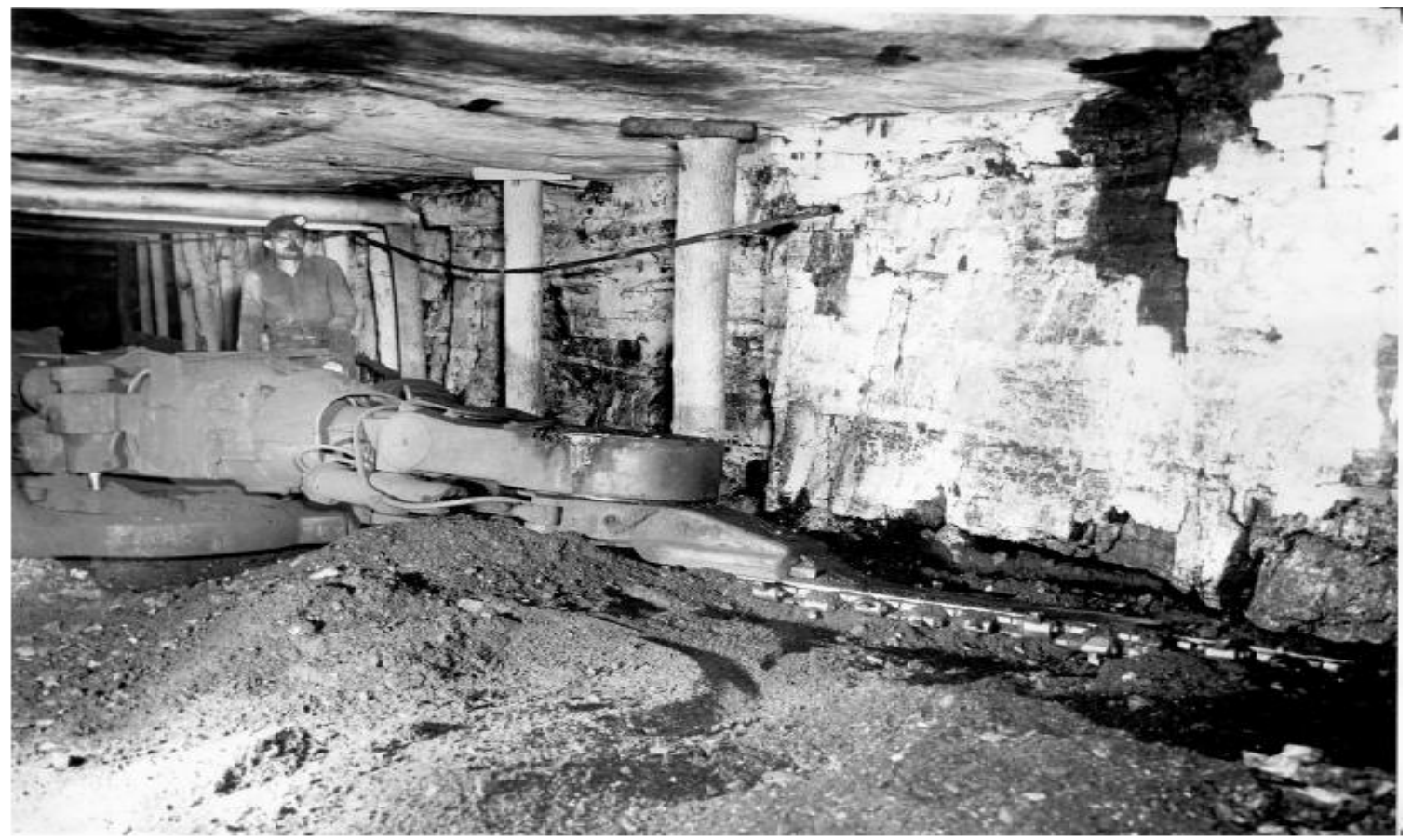

Figure 2.2. Early Coal Cutter (Courtesy of www.coalwoodwv.com)

After the coal was undercut, a mobile coal drill was brought to the face in order to prepare holes for blasting (Figure 2.3). The coal drill was a rubber-tired vehicle with an auger-type drill mounted on a hydraulic boom which enables the drilling of holes parallel to the entry direction over a range of widths and heights without repositioning of the entire unit; the auger bit length was usually matched to the coal cutter bar length (Stefanko, 1983).

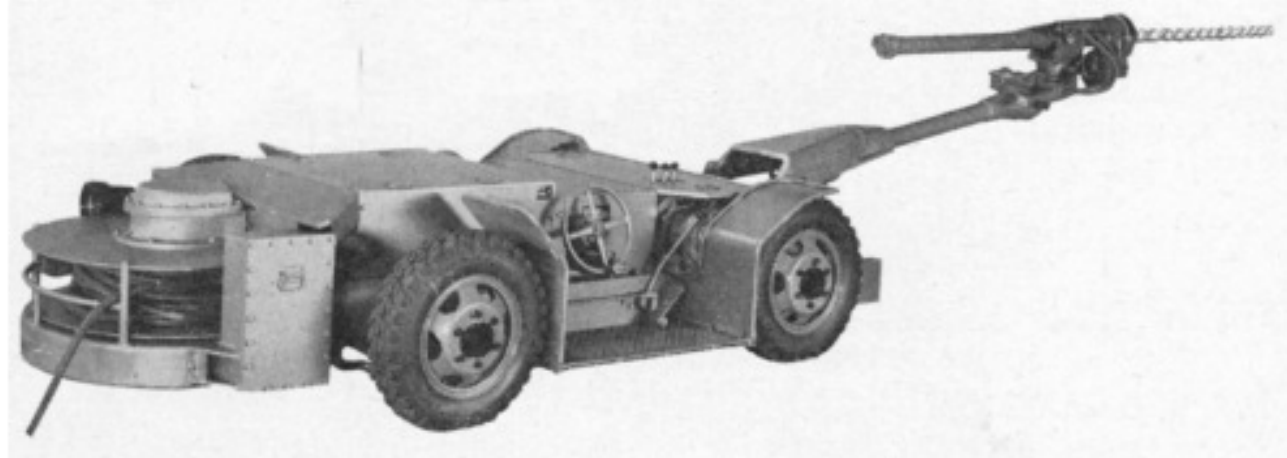

Figure 2.3. Early Coal Drill (Courtesy of www.coaleducation.org)

After the coal face was cut and drilled, blasting operations commenced (Figure 2.4). According to Stefanko (1983), blasting/shooting the coal was accomplished using either permissible chemical explosives or with compressed air. In both cases, the only equipment required were hand tools to load the explosives into the holes and, possibly, a small cart to transport the supplies. 


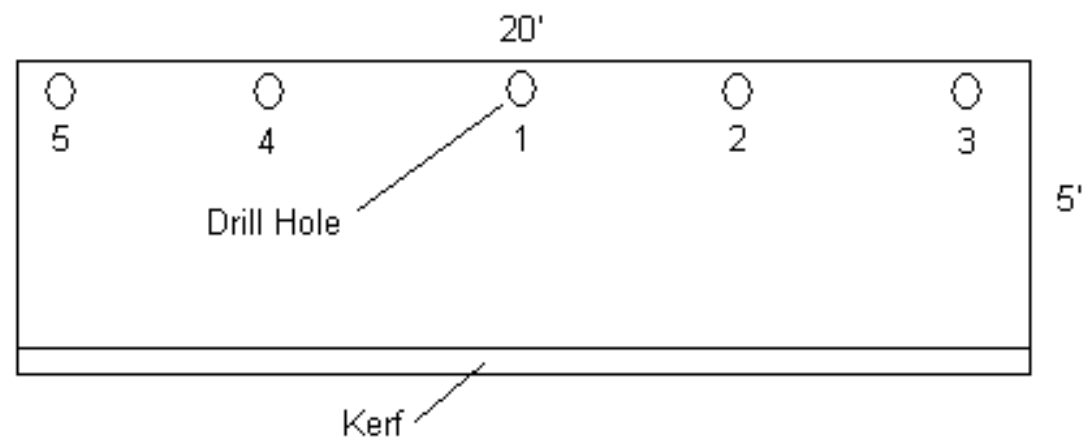

Figure 2.4. Typical Face Prior to Blasting (Stefanko, 1983)

After the coal was shot from the face, it was loaded into a shuttle car or other type of transportation mechanism. A typical loading machine was equipped with two eccentric gathering arms that collected the coal as the machine was advanced into the fragmented pile (Figure 2.5). A chain conveyor then moved the coal from the loading head to the boom on the rear of the machine. The coal was then discharged from the boom to a haulage vehicle (Figure 2.6); in most cases the haulage vehicle was a shuttle car (Stefanko, 1983).

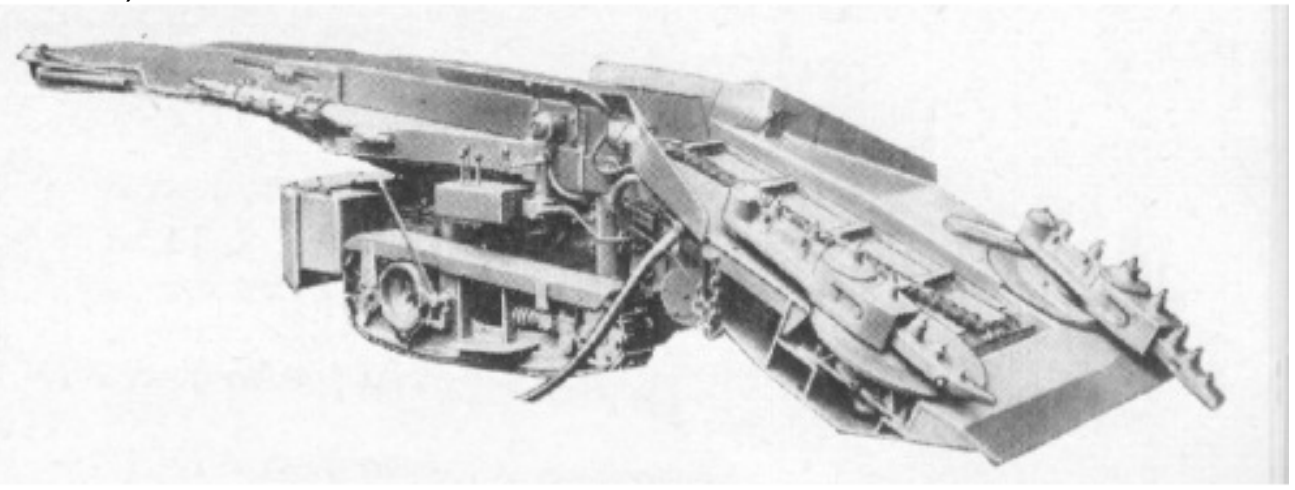

Figure 2.5. Early Joy Loading Machine (Courtesy of www.coaleducation.org)

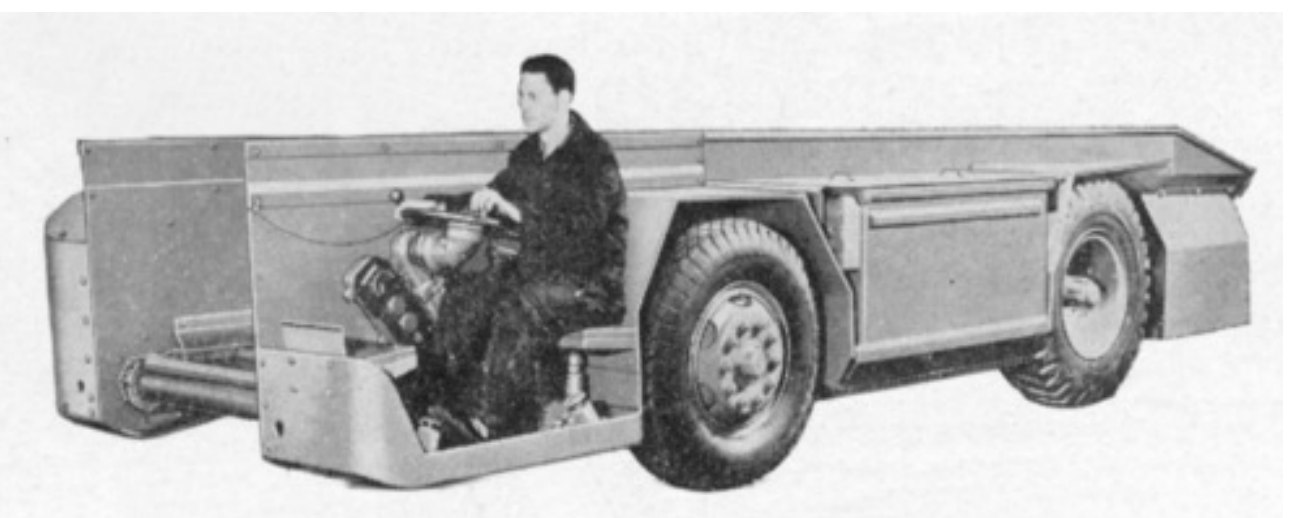

Figure 2.6. Early Joy 42 Shuttle Car (Courtesy of www.coaleducation.org) 
The final step of the conventional mining sequence, roof bolting, was also very similar to what is practiced today in modern mining methods. Stefanko (1983) described roof bolting as the bottleneck in the conventional mining process and stated that it is not uncommon to utilize more than one roof bolter on a conventional section, and that dualboom bolters are commonly used.

Due to the five previously discussed steps required during conventional pillar extraction, conventional pillar mining requires a minimum of five working faces to ensure that all five of the described processes can occur simultaneously but, in most cases, seven working faces were utilized to provide a cushion in case a machine unit should break down. With the two extra working faces, it was possible in conventional pillar mining for a delayed piece of machinery to catch up with the cycle so that maintenance delays did not affect production as drastically as is observed in continuous mining (Stefanko, 1983).

Two common cut sequences associated with conventional pillaring were the open-end method and the pocket-and-wing method. It is important to note that while the open-end method was almost exclusively associated with conventional mining, the pocket-and-wing method, while not common, evolved into a method that could be practiced with modern mining machinery.

Open-End Method. Initially utilized during the hand-loading days before being incorporated into conventional mechanized mining methods, the open-end cut sequence provided a transition which bridged the gap between hand loading and conventional mining. When mining with the open-end method, lifts were mined off the gob side of the pillar (Figure 2.7).

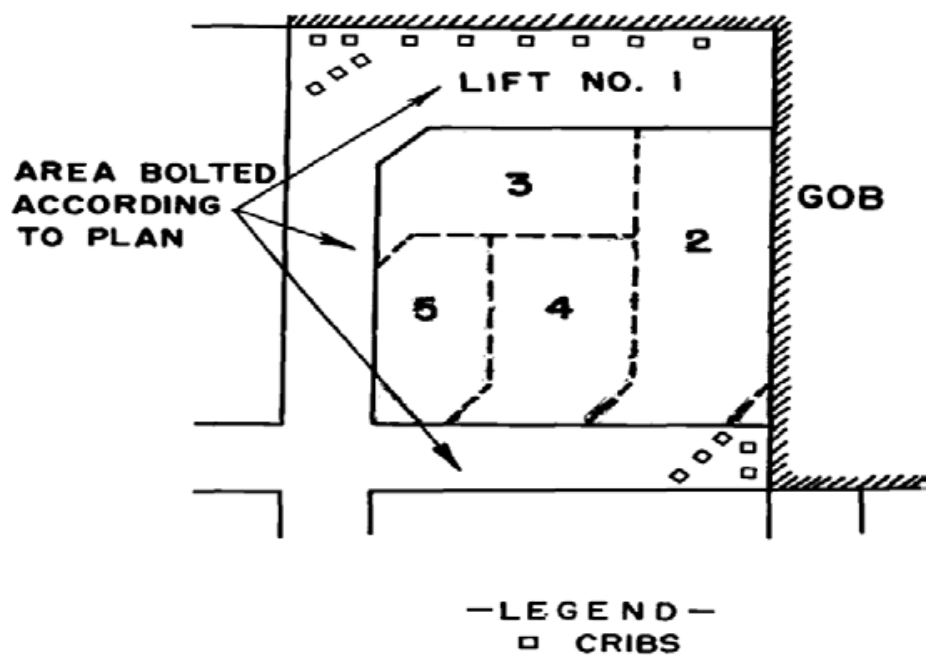

Figure 2.7. Open-End Method (Schroder, 1981) 
Since coal was extracted from the gob side of the pillar, significant additional timber supports, as well as additional roof bolting, were required (Stefanko, 1983). After a pillar was mined, it was necessary to remove the timber supports in order to facilitate proper caving of the gob (Schroder, 1981). When pillaring by the open-end method, pillar lines were developed at 45-degree angles to provide the longest feasible break line, the most possible working faces, and a favorably distributed roof pressure (Stefanko, 1983).

Figure 2.8 represents a typical pillaring section utilizing the open-end cut sequence.

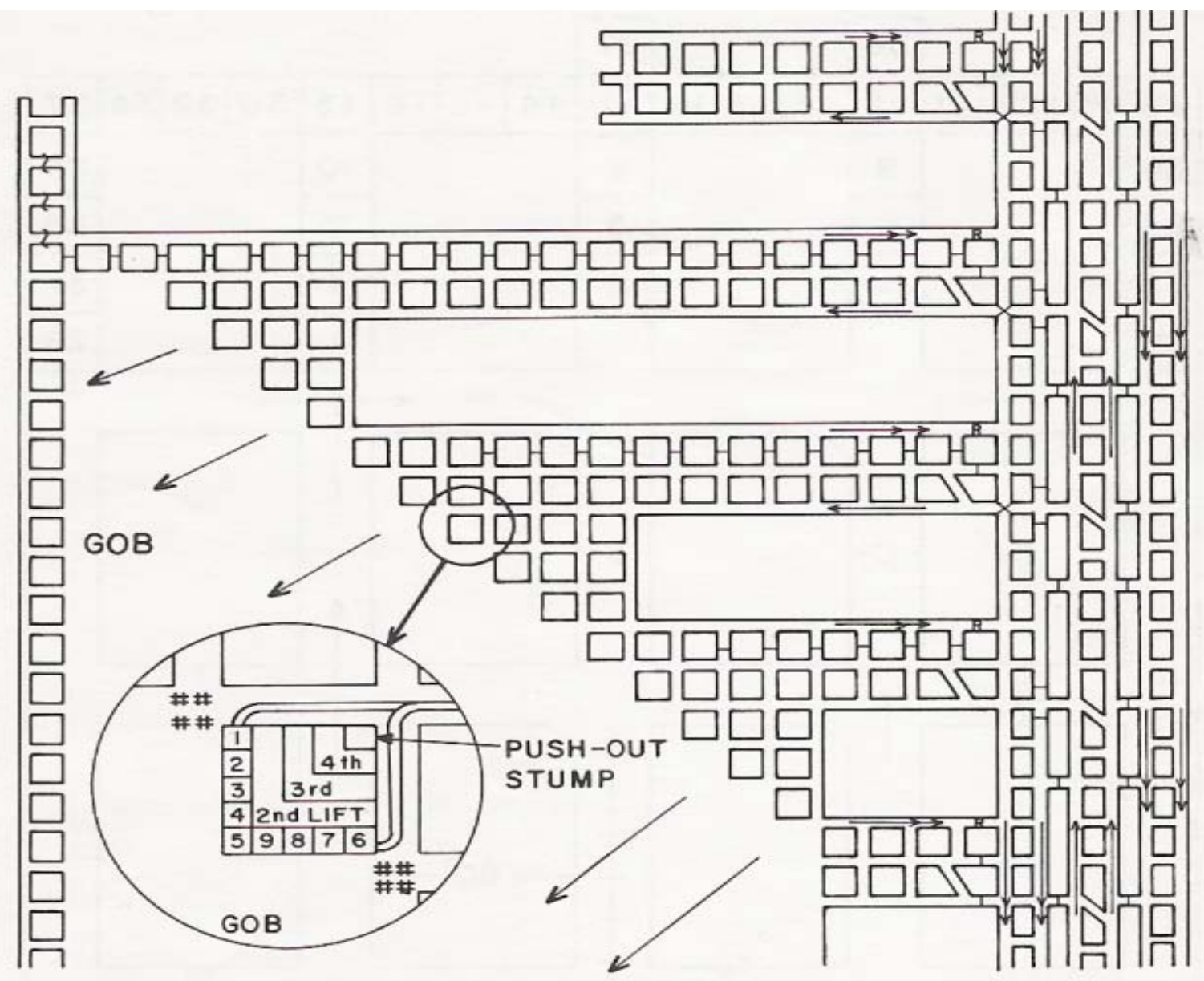

Figure 2.8. Example of the open-end method of pillar extraction (Stefanko, 1983)

As mechanization of the pillaring section continued to advance, the open-end method of pillar extraction became less favorable. This was due to the difficulty of maintaining a 45-degree angle on the pillar line due to the usage of multiple machines at different faces. Also, as mobile machinery became more prevalent (especially loading and haulage machines), accidental dislodging of the numerous timbers required by the open-end method occurred (Stefanko, 1983). Due to its difficulty to adapt to mechanization, excessive exposure of miners to the gob side of the pillar, and the cost of additional roof support, this method is not practiced today. 
Pocket-and-Wing Method. Figure 2.9 represents the pocket-and-wing cut sequence.

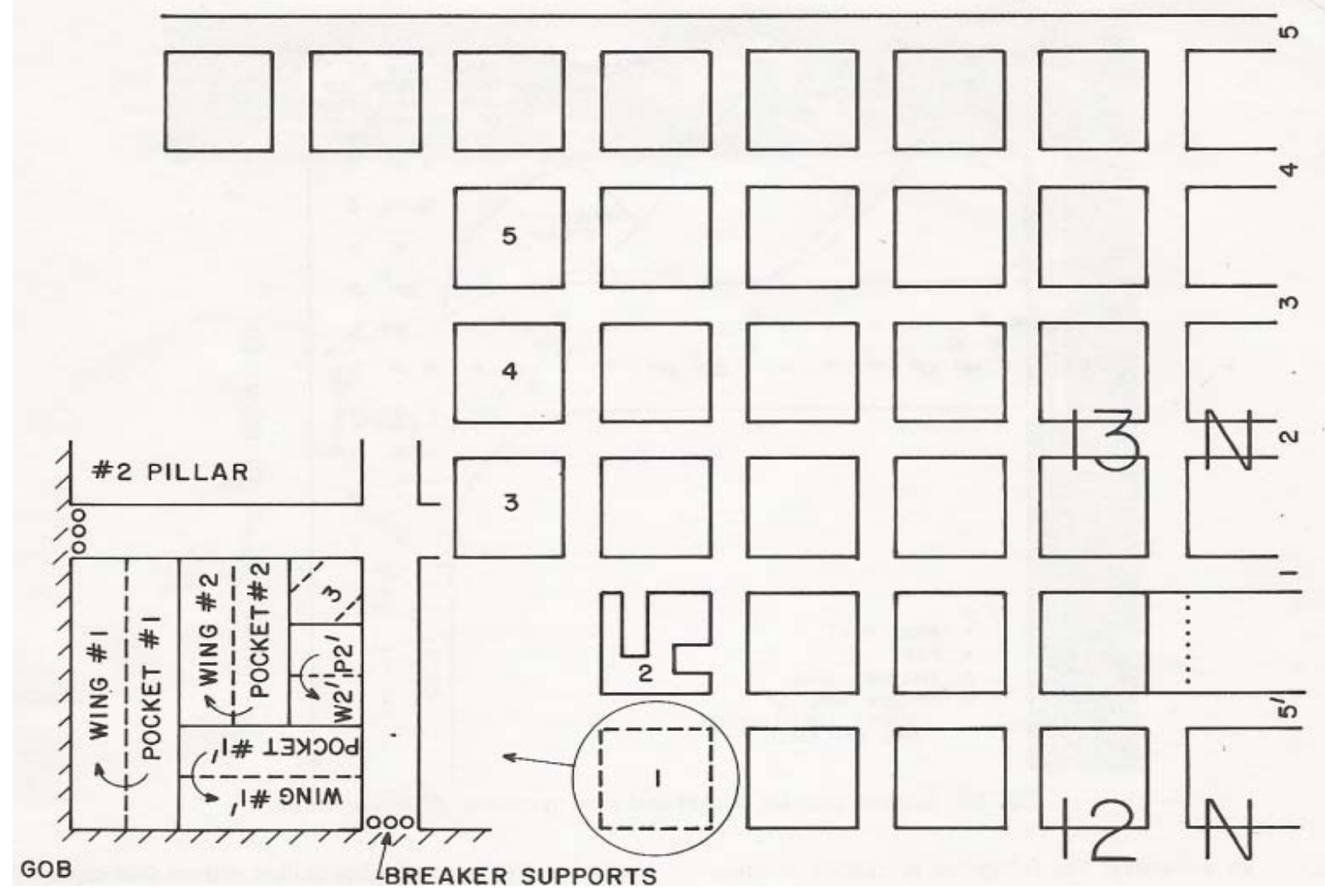

Figure 2.9. Example of the pocket-and-wing method of pillar extraction (Stefanko, 1983)

In the pocket-and-wing method, a lift was mined through the block to the gob but a solid slab of coal was left in place between the opening and the gob. This lift was referred to as the pocket. The remaining solid slab was referred to as the wing. Cuts are then taken from the wing, until the wing was mined out, at which point a new pocket was mined and the process continued until the pillar was extracted (Schroder, 1981). The pocket and wing method was one of the early pillaring methods to transition from conventional mining methods to continuous mining technology that utilized a boring or chain-type continuous mining machine. The continuous miner significantly increased pillaring efficiency by consolidating coal shearing and loading into one machine and reducing the number of operating faces required for machine optimization. Another technological advancement employed by the pocket-and-wing method was the utilization of hydraulic roof support jacks. Hydraulic jacks, serving as breaker posts prior to mining, were positioned in the crosscuts adjacent to the gob and the pillar. Following the extraction of the pillar, trigger mechanisms on the hydraulic jacks were tripped, allowing the jacks to release hydraulic pressure. The jacks were then withdrawn from the crosscuts using an attached cable. Placement of the hydraulic jacks provided roof support superior to wooden timbers and their subsequent removal at the end of the pillar extraction permitted better gob caving and reuse of the jacks (Stefanko, 1983). The use of 
continuous mining machines, recoverable hydraulic roof supports, and minimized gob exposure made this method superior to previous methods. The weakness of this method was that it required additional roof bolting. Since continuous miners were operated from an attached cab, mining regulations required that cuts not be any deeper that approximately $20 \mathrm{ft}$ so, in order to mine through a pillar, additional roof bolting was required. This additional bolting added cost and required that a roof-bolting machine be utilized on the pillar line. The development of the remote-controlled continuous miner enabled significantly deeper cuts to be taken and eliminated the need for additional roof bolting. Thus, the development of the remote-controlled continuous miner was responsible for the decline in utilization of this cut sequence.

\section{Modern Mechanized Pillar Mining}

Modern pillar-extraction methods differ significantly from those used in early mechanized pillar mining. Modern pillaring attempts to minimize miner exposure to gob, and often requires limited or no roof bolting and typically only requires one operating face for optimum efficiency. This is largely due to the development of the remotecontrolled continuous miner, and the mobile roof support. Modern remote-controlled continuous miners and MRS units represent the present pinnacle of pillaring evolution.

Remote-Controlled Continuous Miner. Initial versions of the continuous miner were operated by controls mounted on the machine and, therefore, the allowable depth of cut was limited in order to reduce the exposure of the operator to hazardous roof conditions. The development of the remote-controlled continuous miner and extended cuts permitted cut sequences that required little to no roof bolting. The remote operation also moved the operator further outby, reducing operator exposure to hazardous conditions (Bise, 2007). Figure 2.10 shows the operation of a remote-controlled continuous miner, similar to any that would be found on a modern pillaring section. 


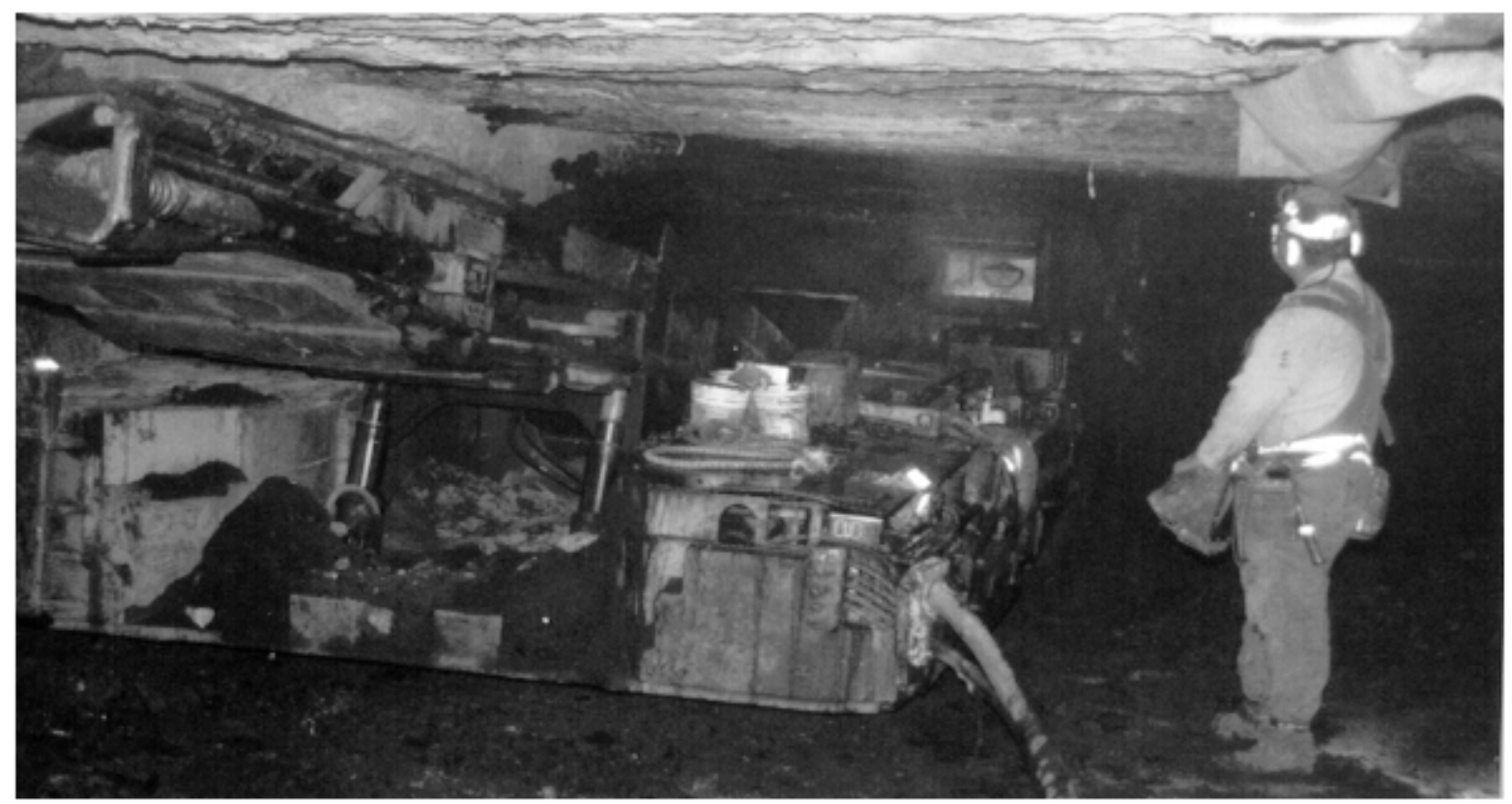

Figure 2.10. Remote Controlled Continuous Miner (Courtesy of www.wvminesafety.org)

Mobile Roof Support (MRS). The most significant advancement in pillaring, in the past 20 years, has been the development of the MRS (Bise, 2007). MRS units are large, mobile, remotely operated hydraulic shields that are pressurized against the mine roof. MRS units (Figure 2.11) are utilized in place of the wing, roadway, and, in some cases, the breaker posts.

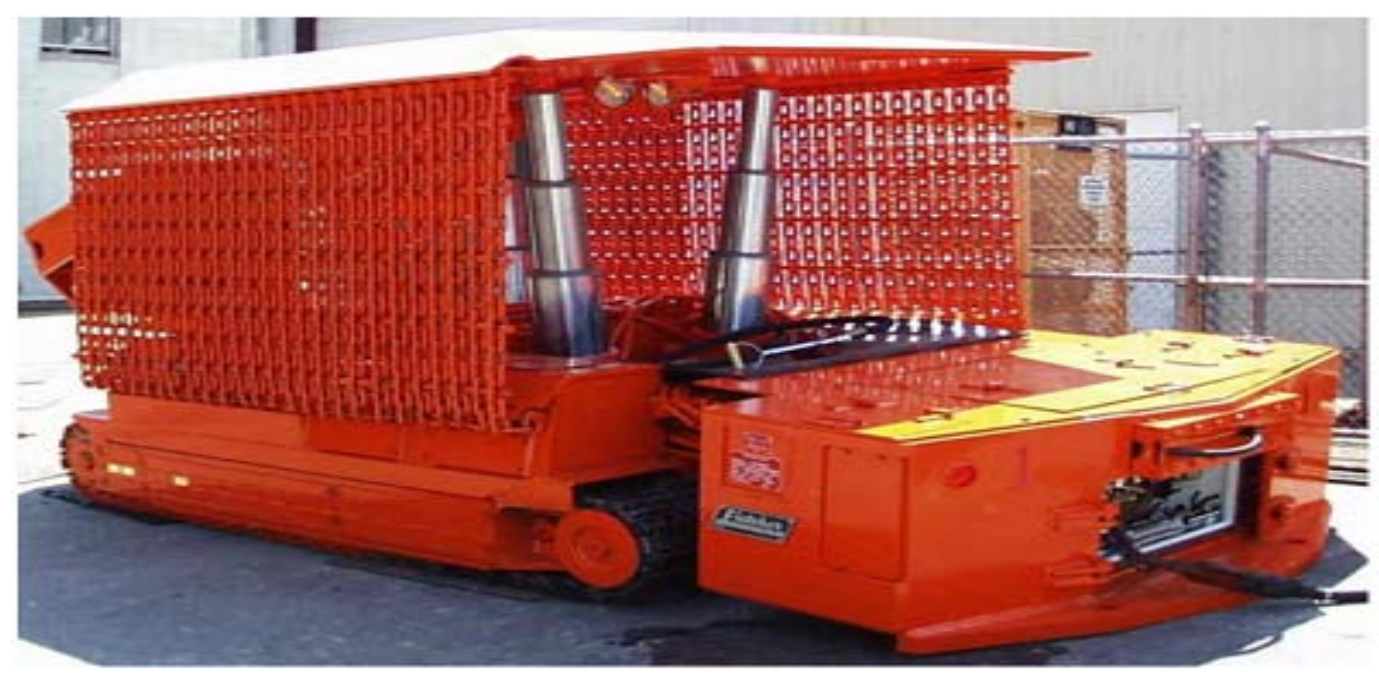

Figure 2.11. Fletcher Mobile Roof Support (Courtesy of Fletcher Mining Equipment)

While there are currently different brands of MRS units in service, the only current manufacturer of MRS units is the J.H. Fletcher Company. New models are designed to operate in seams ranging from 42 in. to $16 \mathrm{ft}$, with load ratings of either 600 or 800 tons, and they provide protection for the mine personnel and the face equipment while pillar 
extraction takes place (Howe, 2008). These units provide greater roof support protection and eliminate or greatly reduce the need for miners to travel close to the pillar line to install the necessary posts for conducting retreat mining. If used properly, the MRS units can provide a safer form of roof support compared to installing posts during pillar mining (MSHA, 2005). Howe (2008) describes the following benefits of MRS units with regard to safety, production, and mine cost:

- MRS usage forces the cave line further into the gob, permitting a wide or thicker fender to be mined on retreat. When radio-controlled continuous miners are utilized, "fenders" as thick as $12.2 \mathrm{~m}$ have been mined on retreat. This eliminates the costly and relatively unproductive cycle of splitting the pillar and the supporting of the pillar split.

- A higher percentage of the reserve can be mined on retreat. Production is derived without the roof support material and the infrastructure costs that accompany mine development and receding faces. Dramatically lower overall cost of production is realized.

- The exposure of workers that are associated with setting turn posts and, in some regions, breaker timber, is eliminated. Radio remote control of the MRS and the proper installation of cable hangers that can break away from the roof can ensure this. Where breaker posts are set, an MRS can be utilized to protect those setting these posts. It is the opinion of many mine personnel that the benefits of breaker posts are not offset by the hazard and expense of installing them. An increase in the overall level of safety may be achieved by not setting this support and keeping the workforce out of the area. However, in those seams where overrunning caves are a factor, breaker posts should be maintained.

- The continuous miner produces at a higher average rate. With older, timber retreat plans, coal fenders were only 4.6 to $5.5 \mathrm{~m}$ wide and the continuous miner spent a large portion of its production cycle turning into the lift, with the cutter drum only partially engaged. The thin fenders were then mined through quickly. The miner operators spent the majority of the shift in the unproductive cycle of turning the miner into the coal rib. With MRS units, the continuous miner is able to mine a large volume of coal without tramming to another place.

- Operating the supports realizes a dramatic reduction in the usage of timber and the accidents that are often associated with timber handling. This includes the cost of the posts and the labor to supply and set the prop. The operation of MRS units eliminates the need for roadway, wing, and in some regions, breaker posts.

- Less time is required to set up for a lift with MRS units than when setting wing posts. Especially when mining lifts both left and right, the MRS units can be advanced before the miner is ready to turn the next lift. Depending on the mining cycle utilized, the repositioning of Mobile Roof Supports is 4 to 5 times faster than timber setting. 
- MRS units permit higher reserve recovery. A higher percentage of the coal in the pillar is recovered safely with MRS units than with timber, because a larger pillar can be safely and more completely extracted. Fewer stumps are left and those that are left are smaller. Many operations profess to achieve a high pillar extraction rate without supplemental support. Observation of the mining in these sections utilizing timber, or no support, indicates that large amounts of resource are left behind. In some situations, this incomplete recovery causes severe strata instability as a direct resulting from incomplete caving of the roof. Total reserve recovery can be realized as high as $85 \%$ when utilizing the roof supports. When compared to the recovery of 45 to $60 \%$ for developing rooms only, this can be a significant addition to mine life. Actual in-pillar recovery can be above $90 \%$. However, pillars must be left in entries and submains development. This reduces overall reserve recovery.

- Complete pillar recovery and the ability to mine a larger pillar results in a more stable pillar line. Generally, the sections utilizing mobile roof supports do not have rides, squeezes, and pressures overriding the immediate face. This can add a substantial factor of safety for the work force, and smooth production from the pillar line. Observations of pillar extraction on the same section before the use of the roof supports, and after the utilization of them, has shown a dramatic reduction in roof and floor heave problems and sharply higher stability of side walls. All this occurs in areas that mine personnel must spend their working shift.

- Fewer personnel are required on a crew retreating with MRS units than on a developing panel, or on a retreat panel with timbering.

- Due to the more complete recovery associated with the operation of roof supports and the larger pillar size, caving becomes more regular and somewhat predictable. The hazards associated with unexpected or premature caving are substantially reduced. However, no guarantees are made or implied in this matter. Caving patterns are, by nature, individual to each mine and can vary according to the strata present at any given location. Variations in the mine roof must be noted by crews and supervision with in-mine experience.

- When unexpected caving does occur, the fall is stopped with a very high degree of reliability by the MRS units. Roof falls which would have caught the continuous miner and require two days to a week to recover, are reduced to no delay or less than two hours for recovery. The recovery process with MRS units is also much safer than with posts and cribs. MRS units have often been used as auxiliary support in these situations, both to increase the level of safety and to speed the recovery process.

- Daily production can be mined at a higher prep plant yield on retreat. The support provided by the MRS units, combined with a more stable pillar line contributes to less deflection in the roof, minimizing the contamination of the 
product by out of seam contamination. A more stable pillar line can decrease floor heave and minimize the need to load floor material.

While the list of benefits is long, operating the MRS units and the withdrawal of the units from the active pillaring area can be extremely hazardous if proper procedures are not followed (MSHA, 2005). Howe (2008) lists the following factors that must be recognized and overcome for reliable and safe operation of the pillar line while utilizing MRS units:

- Always position the MRS units as close as possible to the continuous miner for maximum protection of the equipment and personnel. Do not try to mine more than one lift without advancing the pair of MRS units. However, allow adequate space for the miner operator to rapidly exit the lift and ensure that the miner is not "wedged" by rib sloughage. Do not use them separately. Work the MRS units in pairs only.

- Retraction of the roof plate (canopy) can trigger a fall. Do not lower more than one MRS roof plate at a time when advancing the machine to another lift. If this occurs, or caving begins while advancing the support, reset the roof plate immediately and let the support "ride out" the fall.

- The lack of timber (breaker posts are removed when the MRS units are first positioned in the entry) does not result in the warning that is given in a full timber plan by timber failure. This circumstance can create discomfort among those accustomed to using wood.

- Since a very high percentage of the pillar is recovered with MRS units, it is sometimes advisable to leave a small stump, or fender of coal to give a visual warning or protect the equipment, especially while moving out of the pushout area. These pushout stumps must be sized to crush out later. Each mine represents a different situation regarding the size and placement of these protective stumps. 
Pillaring Methods. Table 2.1 compiled by Mark, et al. (2003), using data supplied by MSHA, shows production and injury statistics for coal mines across the United States. Based on the information presented in this table, it can be concluded that approximately $63 \%$ of 2001 pillaring production was accomplished using the Left-Right cut sequence, commonly referred to as a Christmas-tree sequence (Figure 2.12). Another $32 \%$ was produced using the outside-lift cut sequence while cut sequences, such as split and wing (Figure 2.13), pocket and fender (Figure 2.14) and other variations, likely accounted for the remaining $5 \%$ of production. While this chart provides production and accident statistics related to specific cut sequence, it also provides injury statistics related to the use of MRS units. According to Table 2.1, sections that employ MRS units have a slightly higher accident rate. MRS units are generally assumed to promote safer working conditions and therefore further investigation into the usage of MRS units is likely necessary.

Table 2.1. Demographics of pillar recovery in the US in 2001 (Mark, et al., 2003)

\begin{tabular}{|c|c|c|c|c|}
\hline Mine Grouping & $\begin{array}{l}\text { Summed hours } \\
\text { (millions) }\end{array}$ & $\begin{array}{l}\text { Summed tons } \\
\text { (millions) }\end{array}$ & Tons/hr & $\begin{array}{l}\text { Ground fall } \\
\text { injuries } / 200,000 \mathrm{hrs}\end{array}$ \\
\hline Longwall Mines & 30.33 & 191.20 & 6.31 & 0.81 \\
\hline Room-and-Pillar, Non-Retreat & 12.42 & 56.10 & 4.52 & 1.79 \\
\hline Room-and-Pillar, Retreat & 25.99 & 108.00 & 4.16 & 1.60 \\
\hline All Mines & Total $=74.36^{1}$ & Total $=379.6^{1}$ & Avg. $=5.1$ & Avg. $=1.35$ \\
\hline \multicolumn{5}{|l|}{ Type of Retreat Mining } \\
\hline Full Pillar Recovery & 15.14 & 68.39 & 4.52 & 1.85 \\
\hline Partial Pillar Recovery & 6.35 & 22.15 & 3.49 & 1.29 \\
\hline Both Full and Partial & 4.49 & 17.47 & 3.89 & 1.20 \\
\hline \multicolumn{5}{|l|}{ Cut Sequence } \\
\hline Left-Right & 8.77 & 41.18 & 4.70 & 2.14 \\
\hline Outside Lift & 4.90 & 20.65 & 4.21 & 1.10 \\
\hline Other Known & 0.84 & 3.24 & 3.85 & 2.61 \\
\hline \multicolumn{5}{|l|}{ Pushout Recovery } \\
\hline Recover Pushout & 7.25 & 33.97 & 4.69 & 2.07 \\
\hline Do Not Recover Pushout & 14.66 & 56.14 & 3.83 & 1.35 \\
\hline \multicolumn{5}{|l|}{ Mobile Roof Supports } \\
\hline R\&P Retreat, With MRS & 8.96 & 42.16 & 4.71 & $\underline{1.67}$ \\
\hline R\&P Retreat, Without MRS & 12.28 & 46.07 & 3.75 & 1.53 \\
\hline
\end{tabular}

${ }^{1}$ Totals include contributions from room and pillar mines whose retreat status is unknown

The cut sequence seen most often in Central Appalachia is the Christmas-tree method. The Christmas-tree method is advantageous, due to the fact that it typically requires no additional roof bolting. Also, since pillars are mined from both sides, it is possible to extract wider pillars using this method. While it is the most common, the Christmas-tree method does have drawbacks. According to previous research by Mark, et al. (2002), the Christmas-tree method has some severe disadvantages, such as:

- wider unsupported spans are mined,

- more time is spent in the same location, and 
- the continuous-miner operator's location is not optimal for mining either the leftside or the right-side cut.

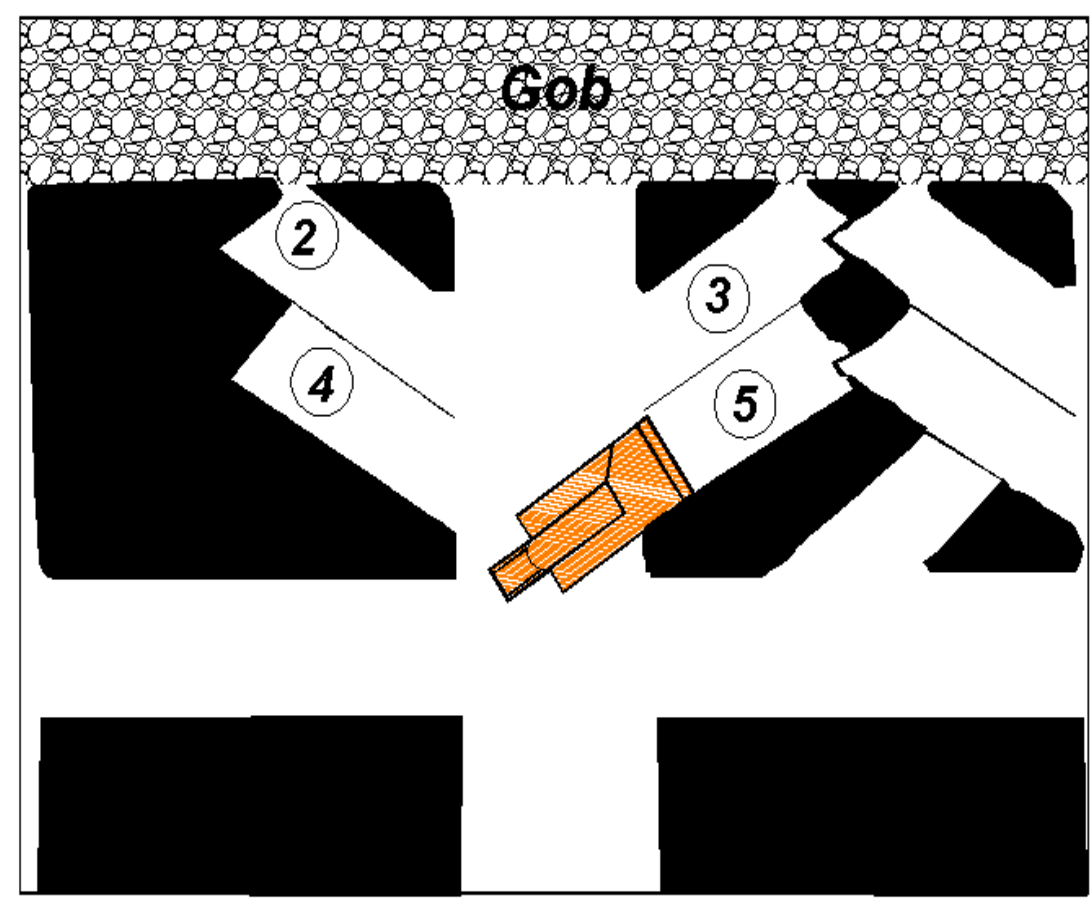

Figure 2.12. Example of the Christmas-Tree Cut Sequence (Mark, et al., 2002)

The outside lift method (Figure 2.13) is the second most utilized modern pillaring cut sequence according to Mark, et al., 2002. 


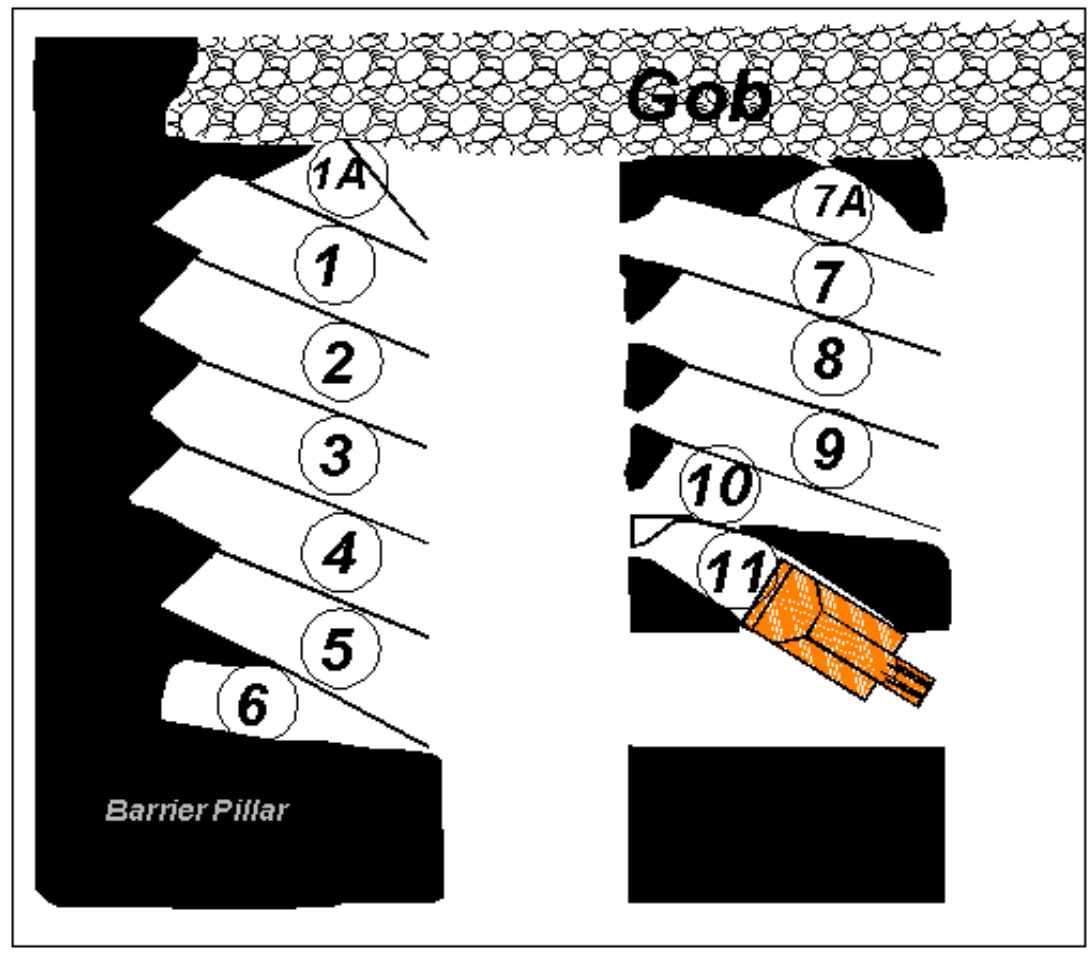

Figure 2.13. Example of the Outside-Lift Cut Sequence (Mark, et al., 2002)

The outside-lift method also has particular advantages and disadvantages. Like the Christmas-tree method, the outside-lift method requires no additional roof bolting. Another advantage of the outside-lift method is the positioning of the continuous miner operator. In this cut sequence, the operator always has a solid coal pillar at his back. Local stability is enhanced, due to the fact that unsupported spans are minimized, in comparison to the Christmas tree sequence, by mining one pillar at a time and leaving a solid pillar adjacent to the one being mined. Also, this method extracts a very high percentage of the pillar. However, there are several drawbacks to the outside-lift method. In order for the outside-lift method to optimize extraction percentages, the allowable depth of cut must be equal to, or greater than, the pillar width. This usually limits its usage to shallow seams, where pillar dimensions are typically narrower (Kauffman, et al., 1981). If the outside-lift method is used in thick seams or under deep cover where large pillars are used, a large remnant pillar must be left. It also requires taking a deeper cut than the Christmas tree method. This deeper cut makes the continuous miner much more difficult to retrieve if it is trapped by a roof fall (Mark, et al., 2002).

Other cut sequences, such as the split-and-wing (Figure 2.14) and pocket-andfender (Figure 2.15), have been utilized in Central Appalachia, but to a minimal extent, as indicated in Table 2.1. This is presumably due to the requirement of additional roof bolting and the subsequent additional cost of the roof bolting. Due to their continued existence, they were included in this thesis but, because they are seldom used in Central Appalachia, they will not be discussed any further. 


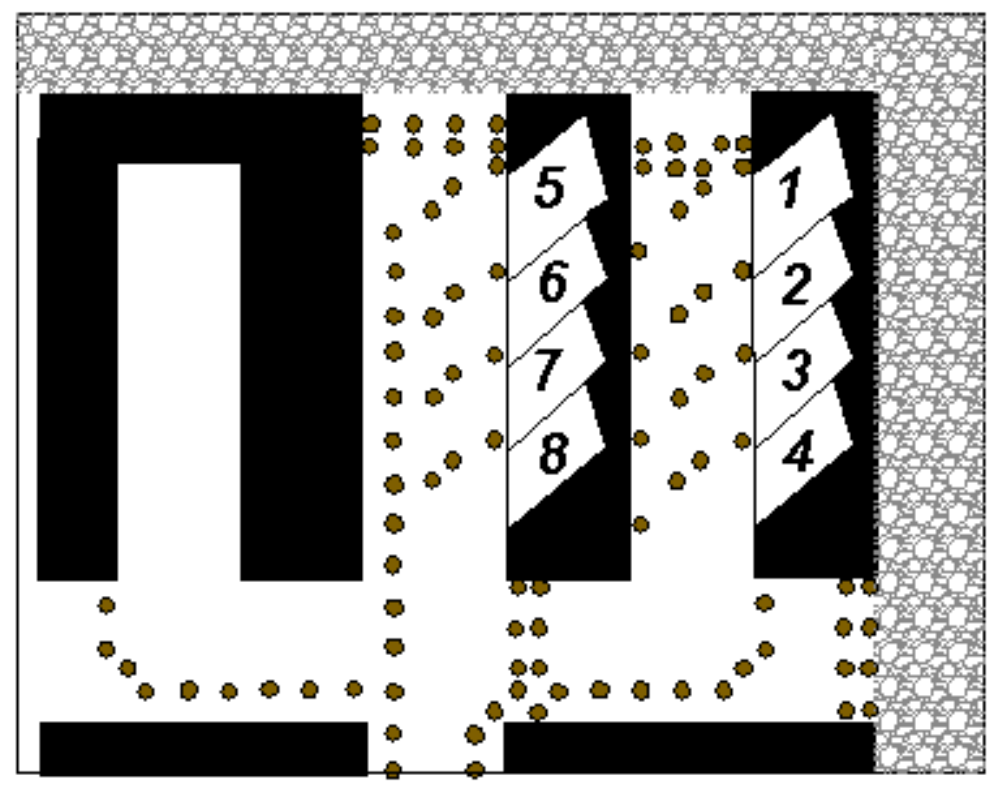

Figure 2.14. Split-and-Wing Cut Sequence (Mark, et al., 2002)

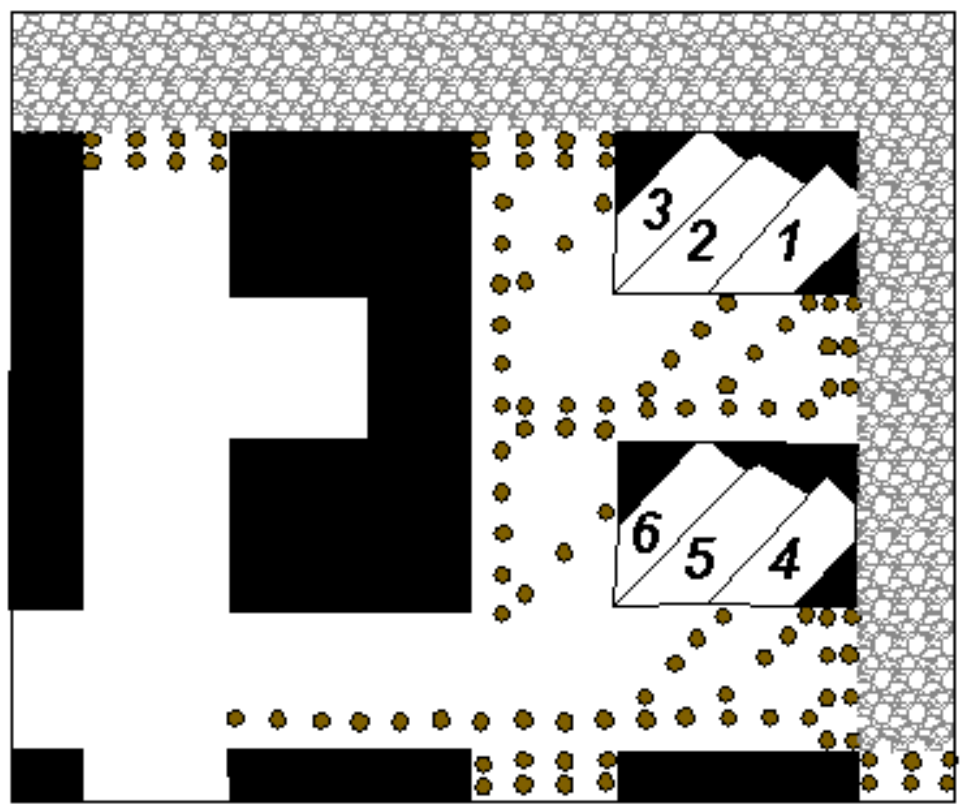

Figure 2.15. Pocket-and-Fender Cut Sequence (Mark, et al., 2002)

\section{Geotechnical Aspects of Pillaring Risk Factors}

In previous research by Mark, et al. (2002), pillaring risk factors have been categorized as global stability factors, associated with the prevention of section-wide 
pillar failure, or local stability factors, associated with the prevention of roof falls in the working area

\section{Global Factors}

During pillaring operations, the support pillars are systematically removed and the load they previously supported is transferred to the surrounding pillars and, to some extent, the gob. It is the additional loading of the surrounding pillars that makes proper pillar sizing so critical.

Squeezes. Squeezes can develop in undersized pillars when they are subjected to loadings that are transferred when inby pillars are removed (Mark, et al., 2003).

Squeezes, also referred to as pillar rides or runs, are described by Chase, et al. (2002) as non-violent gradual failures of coal pillars, resulting in significant sloughage, and roof-to-floor convergence. Since pillar squeezes occur gradually, they are less likely to cause injury than more rapid types of pillar failures, but they are capable of disrupting mining and, in a worst-case situation, driving mining out of the section.

Massive Collapse. Massive collapses can occur in cases where pillars are significantly undersized, or the pillars are not able to support the additional stress caused by pillaring. They fail rapidly and immediately shift the load to the next undersized pillar, causing a chain reaction that can involve very large areas. While a massive collapse, alone, can be considered a catastrophic failure, the resulting air blast can also have devastating and lethal consequences, (Mark, et al., 2003).

Pillar Bump. Pillar bumps are sudden explosive outbursts of elastically behaving coal pillars that occur when high stresses in a coal pillar cause it to violently rupture without warning (Bise, 2007). Pillar bumps arise when coal pillars are too large to adequately yield (shed load) and, too small to withstand the applied loadings (Chase, et al.,2002) According to Mark, et al. (2002), the majority of pillar bumps occur when mining takes place at depths greater than $1,000 \mathrm{ft}$.

\section{Local Stability Factors}

Local stability depends on providing adequate support to the immediate roof in the working area. The most critical area is the active intersection just outby the pillar being extracted. This intersection is the weakest location because of its wide span and proximity to the gob (Mark and Zelanko, 2005). According to Mark, et al. (2003), there are four critical factors affecting local stability:

- Cut sequence,

- Final stump,

- Mobile roof supports vs. Timbers, and

- Roof bolting. 
While the previously listed factors are considered to be the four most critical to local stability, Mark, et al. (2003), also lists the following geotechnical factors affecting the safety and successful operation of a pillaring section:

- Roof geology,

- Intersection span,

- Depth of cover,

- Multiple seam interactions,

- Recovery of older or non-uniform pillars, and

- Continuous haulage, as it impacts entry and pillar design.

Cut Sequence. As previously discussed, the two most common methods used for fullpillar extraction in Central Appalachia are the Christmas-tree and outside-lift methods. Based on the boundary-element numerical-model (BESOL) study conducted during research by Mark, et al. (2002), the outside-lift method was shown to promote the most stable conditions. However, as previously discussed, the outside-lift cut sequence is limited by the width of pillars it can efficiently mine. The benefit of increased local stability is often sacrificed by the selection of a cut sequence that is more capable of yielding high extraction percentages on large pillars, such as the Christmas-tree method.

Final Stump. When left unmined, the final stump provides support for the active intersection (Figure 2.16). If the final stump is removed or undersized, the active intersection may become unstable. Final stump removal is a very high-risk activity that has been practiced not only to recover more coal but also because operators suspected that leaving a stump would prevent the gob area from caving, and might also reduce higher stresses from being transferred to the remaining pillars. Recent experience (Table 2.2) suggests that fears about stump removal may have been exaggerated (Mark, et al., 2003). Nonetheless, during recent mine visits conducted as part of this thesis and discussed in the next chapter, miners continue to express concern that not mining the final stump does prevent proper caving. 


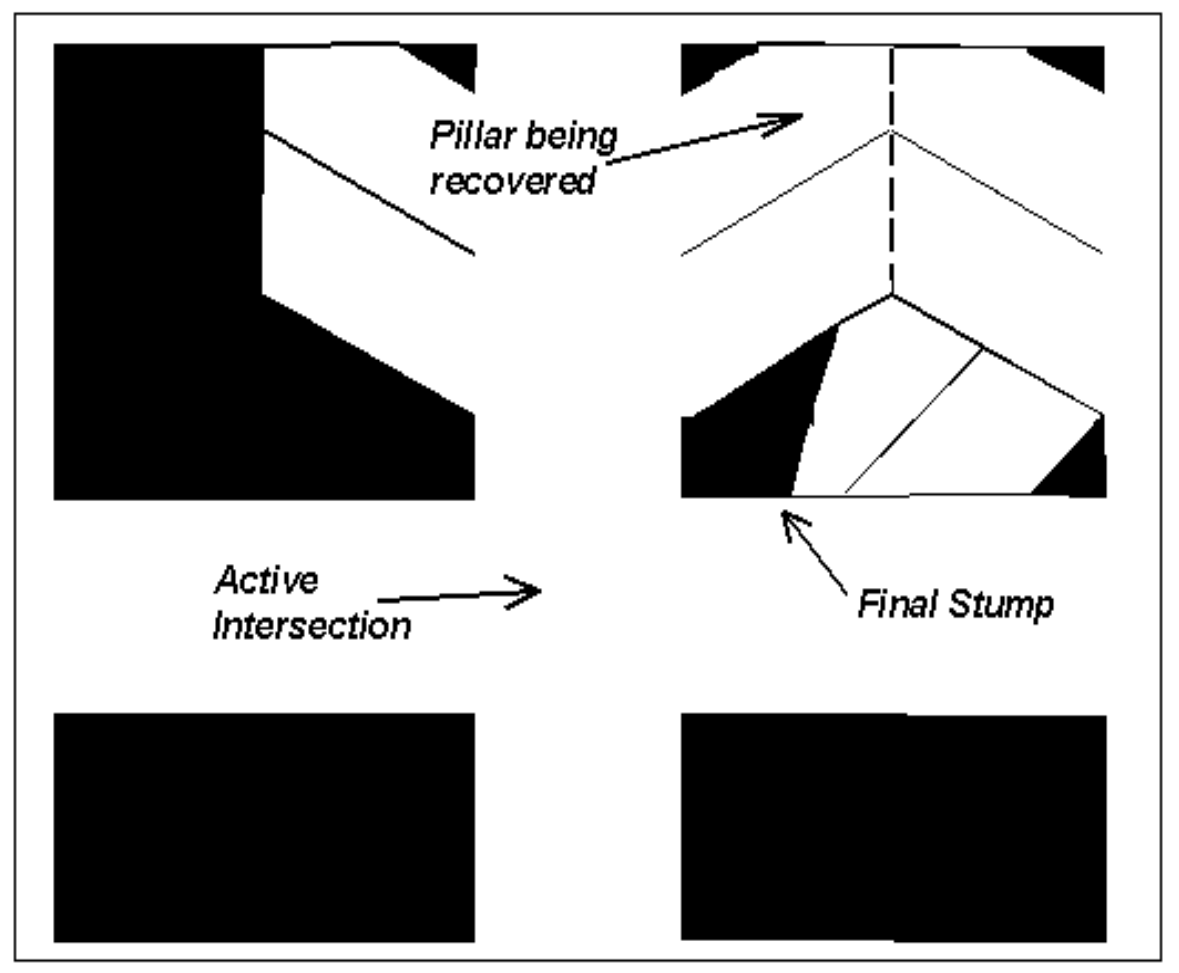

Figure 2.16. Final Stumps (Mark and Zelanko, 2001)

Table 2.2. Guidelines for sizing the final stump (Mark and Zelanko, 2001)

\begin{tabular}{|cc|}
\hline Seam Height $(\mathrm{ft})$ & Stump Size $(\mathrm{ft})^{\star}$ \\
\hline 4 & 8.5 \\
6 & 9.5 \\
8 & 10 \\
12 & 10.5 \\
\hline * Cut-to-corner distance & \\
\hline
\end{tabular}

Mobile Roof Supports vs. Timbers. The traditional supplemental roof support for pillaring has been the timber post; however, the timber post has some severe disadvantages. Due to the weak nature of a timber post, large quantities - - -possibly as many as 100- - - are required to extract a single pillar (Mark, et. al., 2003). Setting posts exposes workers to very dangerous roof conditions, and have resulted in many injuries and fatalities. Timber posts will also fail with a relatively small amount of convergence, and have little to no residual strength.

In retreating sections where MRS units are used, the necessity for timbers is greatly reduced, if not eliminated, and often reduces the hazard exposure to miners. Each MRS provides active support in that, instead of waiting to take load from the roof, the MRS applies vertical force to the roof and immediately takes load and maintains its load through a much larger displacement range than a timber. It is remotely operated and enables the operator to remain at a safer distance outby. The use of MRS units also decreases the number of material-handling injuries. One major drawback of the MRS is its cost. The initial cost of the machine is substantial - - -approximately $\$ 2.3$ 
million for a set of four 800-ton units- - - and this high cost makes it necessary to recover the machine should it become buried, further requiring the purchase of additional equipment and supplies (Howe, 2008). So while the MRS definitely reduces the risks associated with pillaring, owning them may be cost prohibitive for smaller operations. The other major drawback is its seam height limitations; the MRS requires a minimum mining height of approximately 42 inches in order to operate (Howe, 2008). Current MSHA guidelines for safe MRS operation are as follows (MSHA, 2005):

- Onboard, manually-operated controls should be "locked out" or under a "bolted down" cover plate to restrict their usage to maintenance and troubleshooting purposes only.

Because onboard, manual controls (sometimes referred to as manual overrides) are intended for maintenance and troubleshooting only, their use should be restricted to an outby area, between solid coal pillars. To prevent unintended use in an active pillaring area, these controls should be "locked out" or under a "bolted down" cover plate. Information on lock-out devices or bolt down cover plates for the manual controls should be obtained from the MRS manufacturers. Even in an outby area, temporary supports should be installed before pressurizing or de-pressurizing the units with the manual controls. Any material, such as thin pieces of rock, on top of the MRS that poses a fall hazard should either be removed or secured against movement prior to manual operation. Also, any roof damage incurred as a result of pressurizing and depressurizing the MRS unit should be addressed before removal of the temporary supports.

- Umbilical remote controls should only be used for tramming the MRS units between solid coal pillars.

Umbilical remote control requires the operator to physically "plug-in" or retrieve the pendent control on the MRS unit. This connection should not be made until the MRS has been moved via the radio remote control into a safe location completely out of the active intersection following the last lift. Once away from the active pillaring area, the pendent can be safely plugged in or retrieved and used for tramming the MRS to the next pillaring location. However, the operator should utilize sufficient pendent cable length to maintain a safe operating distance and should never use the umbilical controlled unit to displace breaker timbers.

- When tramming the MRS units, all personnel should be clear of pinch points between the units and between the units and the rib.

Under no circumstances should anyone, including the MRS operator, be permitted beside or within the turning radius of the MRS units when they are being moved.

- When setting and lowering the MRS, the operator should be positioned in a safe location, at least 20 feet away from the units. 
A greater distance may be required depending on the MRS working height, immediate roof conditions, the amount of accumulated debris atop the units, or the lift being mined. For example, during mining of the last lift the MRS operator should remain out of the active intersection. During the setting and lowering process, all personnel should be positioned outby the MRS operator. This includes personnel assisting the MRS operator by monitoring the pressure gages.

- While in the active pillaring area, the MRS units should be operated in pairs, using the radio remote control.

When moving the mobile roof supports from pillar lift to pillar lift, each MRS should be advanced sequentially such that one unit will never be offset more than one half the length of its companion unit. The MRS units should be advanced immediately after each lift, and should be kept as close as practical to the continuous mining machine during each lift. Upon completion of mining in a given pillar, the MRS units should be moved sequentially until they are between solid coal pillars. During this process, at least one unit will be pressurized against the roof at all times.

- While pillaring, MRS pressure gages or colored area lights should be used to monitor roof loading.

Gages or load indicating lights should be observed to first ensure that the MRS units have been properly set against the mine roof. (Mine management should determine a pressure level for setting the MRS units). Gages or lights are to be continually observed to ascertain if pillaring operations need to be stopped in a specific lift. These gages should be large enough to allow remote monitoring. Mining should cease in a lift either at the MRS yield pressure or at some lower level dictated by roof conditions (determined by mine management). Pillaring operations should also cease if neither the gages nor lights are operational.

- Install the MRS cables with break-away cable hangers so that the cable can be pulled down remotely.

Use of such devices will prevent personnel exposure to hazardous roof adjacent to the MRS units. No hangers should be retrieved from the blocks being pillared.

- Precautions should be included in the Roof Control Plan for supporting the roof in the event one of the MRS units becomes inoperative.

Depending on mine-specific circumstances, the roof control plan may permit the repositioning of one of four units to maintain a pair in the more critical area adjacent to the gob. For example, if an MRS unit in the straight becomes inoperative, an outby MRS from the crosscut could replace it. Wood supports may provide an acceptable replacement for an MRS unit in a breaker setting but 
should not be used in conjunction with an MRS unit in a radius turn post application. It is not advisable to simply substitute wood supports for an MRS and continue the same cut plan/lift sequence.

- When parking the MRS units for an extended period of time, they should be positioned between solid coal pillars outby the active pillaring area. The units should remain in contact with the roof to prevent dynamic loading in the event of a roof fall.

Pressurizing the MRS against the roof in areas where personnel will later be working or traveling may create a hazard by damaging the roof or permanent roof support. Therefore, in this circumstance, the MRS units should be pressurized with minimal force (just against the roof). Also, the radio remote control units should be stored in a safe area away from the machines.

- The roof control plan should include a method for the safe retrieval of a disabled or entrapped MRS.

Train miners before utilizing the plan.

Roof Bolting. Although pillaring sections are considered to be short term and, as a result, are not as densely bolted as areas that are considered more permanent, failure to provide adequate bolting can prove to be a costly mistake. According to Mark, et al. (2002), installing additional roof-bolt support may be the simplest way to prevent roof falls on pillaring sections. Although there is no widely accepted method for designing roof-bolt patterns for pillaring, Mark, et al. (2002), recommends that the NIOSH computer program, "Analysis of Roof Bolting Systems" (ARBS), is appropriate and lists the following as factors for improving roof-bolting systems:

- Longer bolts that build a thicker beam or anchor in more competent roof.

- Stronger bolts, using larger diameter rods or higher-grade steel, that are less likely to break from rock movement.

- Extra intersection support such as cable bolts.

- Point anchor resin-assisted bolts that can provide warning of high loads.

Geologic Factors. Weak rock, such as shales, mudstone, and coal, are more likely to be damaged by the changing abutment stresses that occur during pillaring. They are also more likely to contain slips, slickensides, horsebacks and other discontinuities (Mark, et al., 2003). These conditions may require additional roof bolting, and leaving the final stump in place becomes even more critical. Extensive effort should be made to identify major roof discontinuities, such as slips, slickensides, and horsebacks, before mining. The roof should also be closely monitored during the pillaring process (Mark and Zelanko, 2005).

Intersection Span. Research by Molinda, et al. (1998), collected roof-fall data from 892 mines that experienced 2,824 unintentional roof falls. Of the falls with known locations, 
approximately $71 \%$ were located in intersections. It was also estimated that intersections account for $20-25 \%$ of the total drivage, so it was concluded that roof falls are 8-10 times more likely to occur in intersections. This is due to longer span lengths which can far exceed normal entry widths. The risk is even higher if poor roof conditions exist. The following is a list of steps provided by Mark, et al. (2002), that help to stabilize intersections:

- Minimize entry widths.

- Reduce the number and depth of turnouts.

- Use longer and stronger bolts in intersections.

- Leave an adequate final stump.

- Install extra standing support in the intersection if the final stump is extracted (MRS or roadway posts).

Depth of Cover. As mining depths increase, both vertical and horizontal stresses increase. There is some disagreement about the depth at which cover is considered deep. As previously stated, depths of $1000 \mathrm{ft}$ or more are considered to be in the range for pillar bumps, but others suggest that anything in excess of $750 \mathrm{ft}$ magnifies problems affecting both local and global stability. While there is no clear definition of deep cover, it is widely accepted that, proper pillar design is critical for combating the risks associated with deep cover (Mark, et al., 2003).

Multiple Seam Interactions. Multiple seam interactions commonly occur in Central Appalachia and will become increasingly more common as market prices rise to support the more difficult mining of thinner and more discontinuous coal seams that may have been left behind when a mineral property was previously mined. The two most common hazards associated with multiple-seam interactions identified by Mark, et al. (2007) are the following:

- Undermining, where stress concentrations due to previous full extraction in an overlying seam are the primary concern.

- Overmining, where previous full extraction in an underlying seam can cause stress concentrations and rock damage due to subsidence.

Multiple seam interactions may result in roof falls, rib spalling, floor heave, and pillar bumps (Mark, et al., 2007). Mark, et al. (2003) recommends that "zones of potential interactions should be carefully mapped in the planning stage, and pillar recovery should be avoided where severe interactions are anticipated."

Recovery of Older or Non-Uniform Pillars. With today's coal market, it is becoming feasible to re-open mines to extract their remaining pillars. While these mines may contain significant reserves, it is important to recognize the additional complications of pillaring in old works. Pillaring may not have been intended for these mines when they were initially developed. As a result, pillars may be irregularly sized due to design or degradation. Roof spans may be excessive, and the roof-control system (bolting or otherwise) may require major rehabilitation. Pillaring is safest when the extraction routine becomes uniform and predictable (Mark, et al., 2003). In many cases, due to the 
aforementioned complications, uniformity and predictability in old works is difficult to achieve.

Continuous Haulage. In a continuous haulage system, the pillars are typically retreated from both sides toward the center (Figure 2.17). This results in a pillar point in the middle of the active pillar line. The increased stress on this pillar point, due to the removal of adjacent pillars as well as the increased center entry width to accommodate haulage equipment makes the mining of the two center pillars a very high-risk task. An additional mobile bridge unit should be added to the haulage system, permitting the pillars to be worked from the outby intersection and permitting the unidirectional removal of pillars, thereby eliminating the pillar point. Also, if a continuous haulage system is planned with the intent of extracting pillars after development, then the crosscut angles should be minimized as much as possible (Mark, et al., 2003).

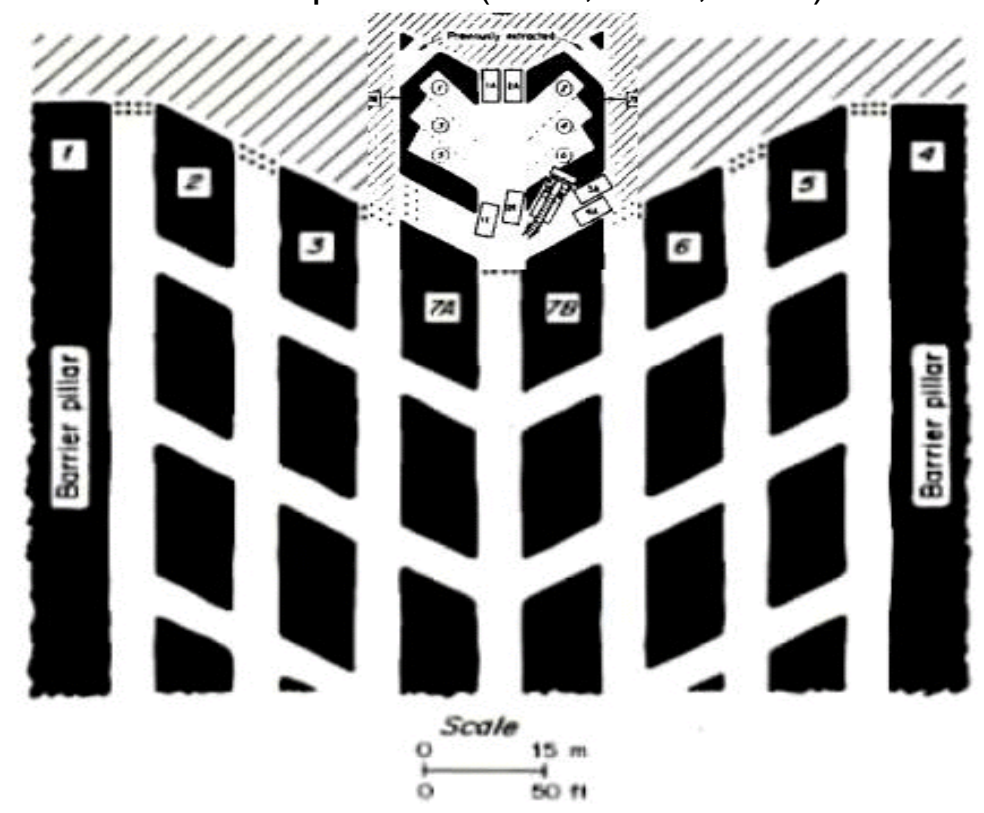

Figure 2.17. Continuous-Haulage Section Layout (Mark, et al., 2002)

\section{Summary}

Geotechnical risk factors have a very significant impact on pillaring. As a result, each of the following risk factors has a tremendous influence on the safety and success of a pillaring operation (Mark, et al., 2007):

- Cut sequence,

- Final stump,

- MRS vs. timbers,

- Roof bolting,

- Geology,

- Pillar age and uniformity and,
- Continuous haulage, as it affects entry and pillar design 
Unfortunately, in Central Appalachia, these risk factors are often encountered in multiple combinations. However, all of these risk factors can be addressed prior to mining. By assessing these risks prior to mining, and continuing to monitor them during mining, it should be possible to improve safety and increase the success rates of pillaring operations in Central Appalachia.

\section{Mineworker Performance on Pillaring Sections}

Significant engineering research has been focused on the geotechnical risk factors discussed in the previous section; further, the importance of those risk factors, as well as the attention they require, cannot be understated. However, geotechnical risk factors are just one side of the equation. Engineering controls, designed to manage geotechnical risk factors can be highly effective, but they do not eliminate risk. There is always residual risk resulting from the limitations of engineering controls. This is evident by the continuing occurrence of injuries and fatalities on pillaring sections. This residual risk must be addressed through training and proper personnel performance. So in order to more comprehensively manage risk, focus must be directed on both geotechnicalbased risk and personnel-performance-based risk. By doing this, it should be possible to further reduce risk and improve the safety and efficiency of pillaring.

\section{Review of Statistics Related to Pillar Extraction}

While there are many potential fatality hazards associated with underground mining, two hazards are intensified by pillaring: 1) fall of roof, and 2) fall of rib. Although pillar bumps are considered to be violent collapses of coal pillars, MSHA continues to categorize bumps as falls of roof or ribs. As a result, this study will follow the same classification. All fatalities reviewed in this section resulted from falls of roof or rib on pillaring sections.

Between 1995 and 2007, a total of 34 fatalities occurred on pillar-extraction sections in the five states that practice pillaring. The five states consist of three in Central Appalachia (West Virginia, Virginia, and Kentucky) and two in the Western United States (Utah and Colorado). During that same time period, there were 89 total fatalities due to falls of roof or rib in those five states. Thus, pillaring accounted for $38 \%$ of the fatalities resulting from falls of roof and rib in the five states where pillaring is practiced.

Figure 2.18 compares the pillaring-related fatality statistics for the five states. It is interesting to observe that pillaring represents approximately $33 \%$ of the fall-of-ground underground-coal-mining fatalities in each of the Central Appalachian states. Production and active mine information is presented in Tables 2.3 and 2.4. 


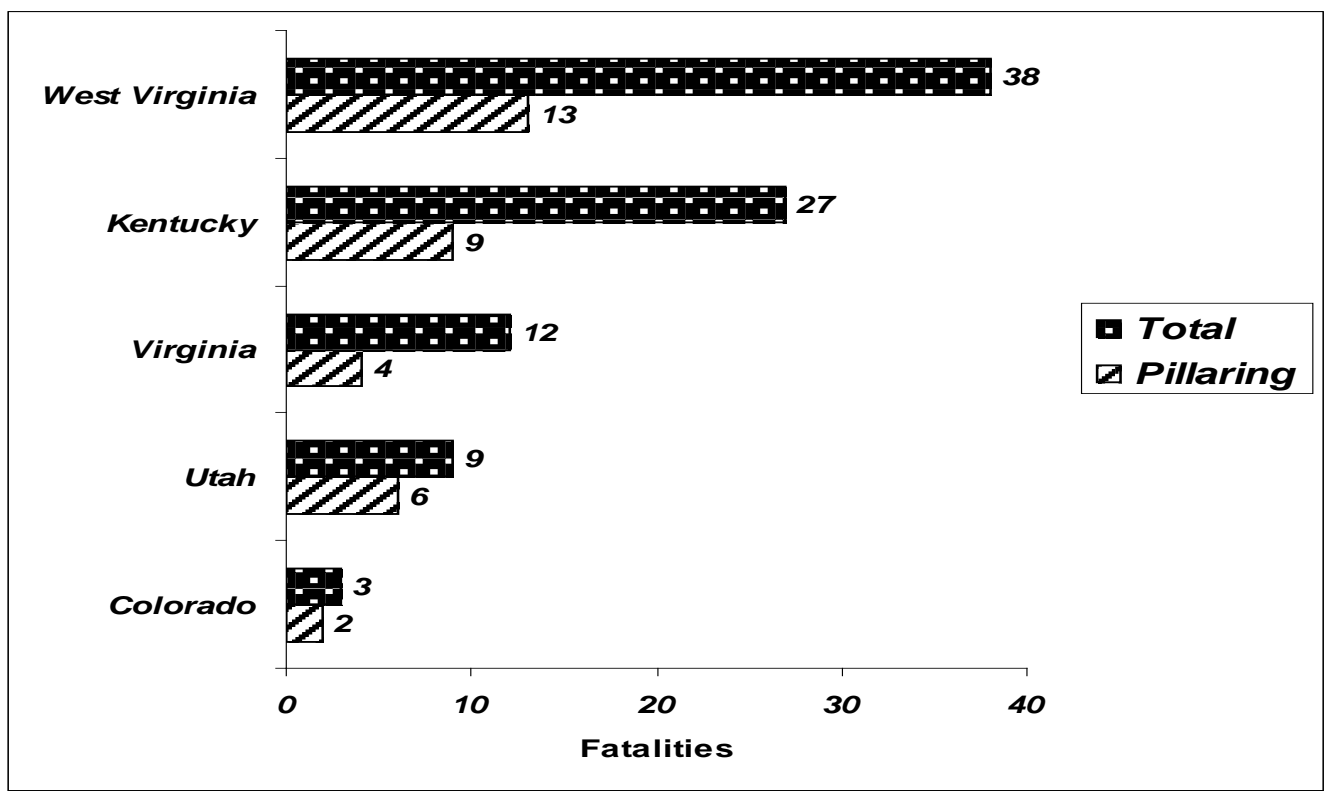

Figure 2.18. Fatalities Related to Falls of Roof or Rib (1995-2007) (MSHA, 2008)

The nine fatalities that occurred in the state of Utah were all related to pillaring. However, three were related to rescue efforts and did not occur while during mining.

Table 2.3. Underground Production, (U.S. Energy Information Administration, 2010)

\begin{tabular}{|l|c|r|}
\hline \multicolumn{3}{|c|}{$\begin{array}{c}\text { Underground Production } \\
\text { (Million Tons) }\end{array}$} \\
\hline & \multicolumn{1}{|c|}{$\mathbf{2 0 0 7}$} & \multicolumn{1}{|c|}{$\mathbf{2 0 0 8}$} \\
\hline $\boldsymbol{W}$ & 84.853 & 88.369 \\
\hline KY & 69.217 & 69.474 \\
\hline VA & 15.371 & 15.806 \\
\hline UT & 24.307 & 24.365 \\
\hline CO & 27.61 & 24.37 \\
\hline
\end{tabular}

Table 2.4. Active Underground Mines, (U.S. Energy Information Administration, 2010)

\begin{tabular}{|c|c|c|}
\hline \multicolumn{3}{|c|}{ Underground Mines } \\
\hline & $\underline{\mathbf{2 0 0 7}}$ & $\underline{\mathbf{2 0 0 8}}$ \\
\hline $\boldsymbol{W V}$ & 168 & 186 \\
\hline $\boldsymbol{K Y}$ & 227 & 216 \\
\hline $\boldsymbol{V A}$ & 71 & 65 \\
\hline $\boldsymbol{U T}$ & 10 & 9 \\
\hline CO & 7 & 8 \\
\hline
\end{tabular}

As part of the pillar-extraction fatality review, all states that experienced a pillaringrelated fatality between 1995 and 2007 were included in the previous section. However, West Virginia, Kentucky and Virginia will be the focus of the remainder of this study. 
This is justified by their location in Central Appalachia, their production levels, and their significantly higher number of active underground mines.

New technologies have been developed that have had major impacts on the mining industry both in the areas of production and safety. The pillaring aspect of mining is no exception. Mobile Roof Supports (MRS) are considered by most to be the most recent significant technology applicable to pillaring. As part of the fatality review conducted during the course of this thesis, fatalities were categorized into two types: 1 ) fatalities where MRS units were utilized and 2) fatalities where MRS units were not utilized. Figure 2.19 and 2.20 show that, although, MRS units do improve safety, fatalities are still occurring, even with the implementation of this technology. The fact that fatalities continue to occur, despite the implementation of advanced technologies such as the MRS, indicates there may be deficiencies in safety training.

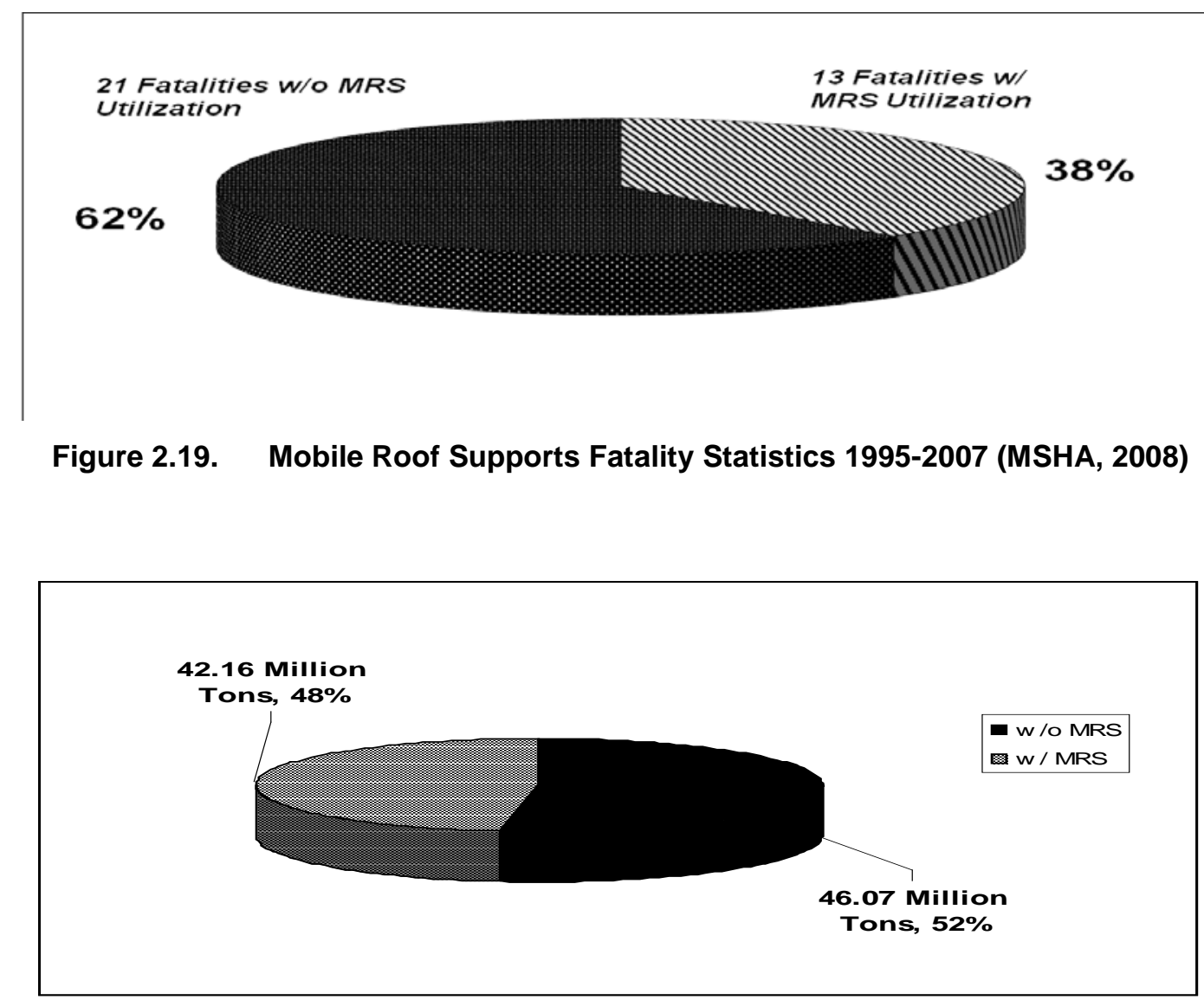

Figure 2.20. 2001 U.S. Pillaring Production (Mark, et al., 2003) 
In order to better understand the risks facing each job description, fatalities were also categorized by job description. Figure 2.21 presents pillaring fatalities between 1995 and 2007 on the basis of job classification.

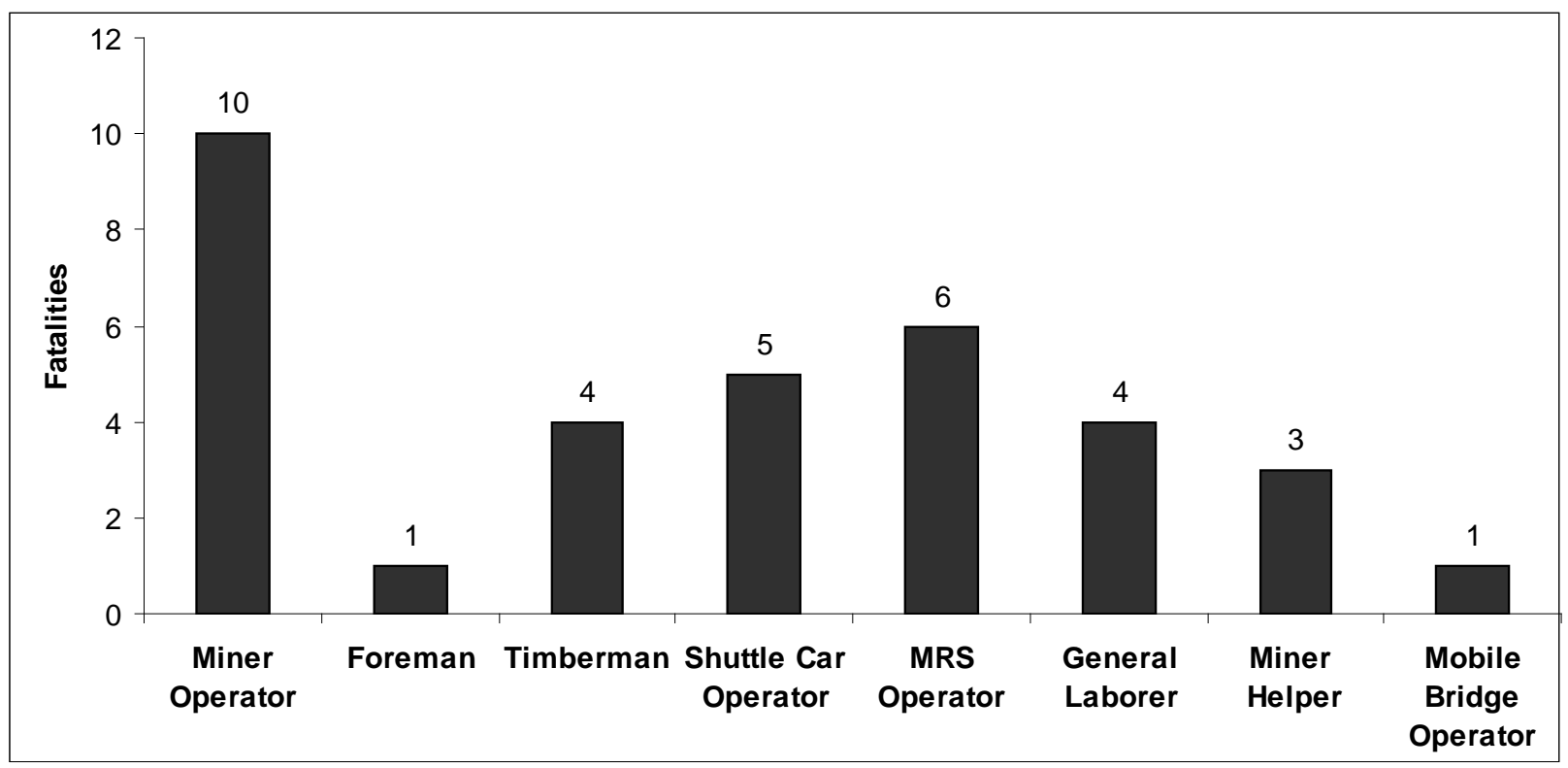

Figure 2.21. Fatality Statistics by Job Description 1995-2007 (MSHA, 2008)

\section{Fatality Case Studies}

Throughout the course of this thesis, emphasis has been placed on the risks and benefits of modern pillar extraction. In order to identify hazards and behavior trends, it was necessary to study pillaring-related fatality reports. During this study, all pillaring related fatality reports from $1995-2007$ were carefully reviewed in order to identify behavior trends and hazards. Of the 34 pillaring-related fatalities studied, five have been selected for presentation in this thesis. These five were selected because they are the best representatives of the hazards and behavior trends that were commonly observed during mine-site observations. All five incidents occurred in Central Appalachia. Fatality reports were collected from MSHA's database and all names, dates and specific locations in the reports have been modified to assure confidentiality. Summaries of the fatality reports have been included in this chapter. The full reports taken from the MSHA database are presented in Appendix A, again with identifying details modified to assure confidentiality. 


\section{Fatality Case Study No.1}

This incident being analyzed represents a double fatality. A section foreman, as well as a continuous miner operator helper (CM helper), were fatally injured as a result of a massive roof collapse. The section foreman and the $\mathrm{CM}$ helper will be referred to as Victim 1 and Victim 2, respectively. The full fatality report obtained from the MSHA fatality database is presented in Appendix A of this thesis.

There are two particular characteristics that make this incident worthy of further investigation: 1) the roof control plan was approved using only timbers as opposed to mobile roof supports, and 2) MSHA determined that there were no violations contributing to the incident. These two characteristics are significant because they support the use of mobile roof supports over timbers, as well as identify the increased risk associated with non-essential personnel congregating in the active mining zone.

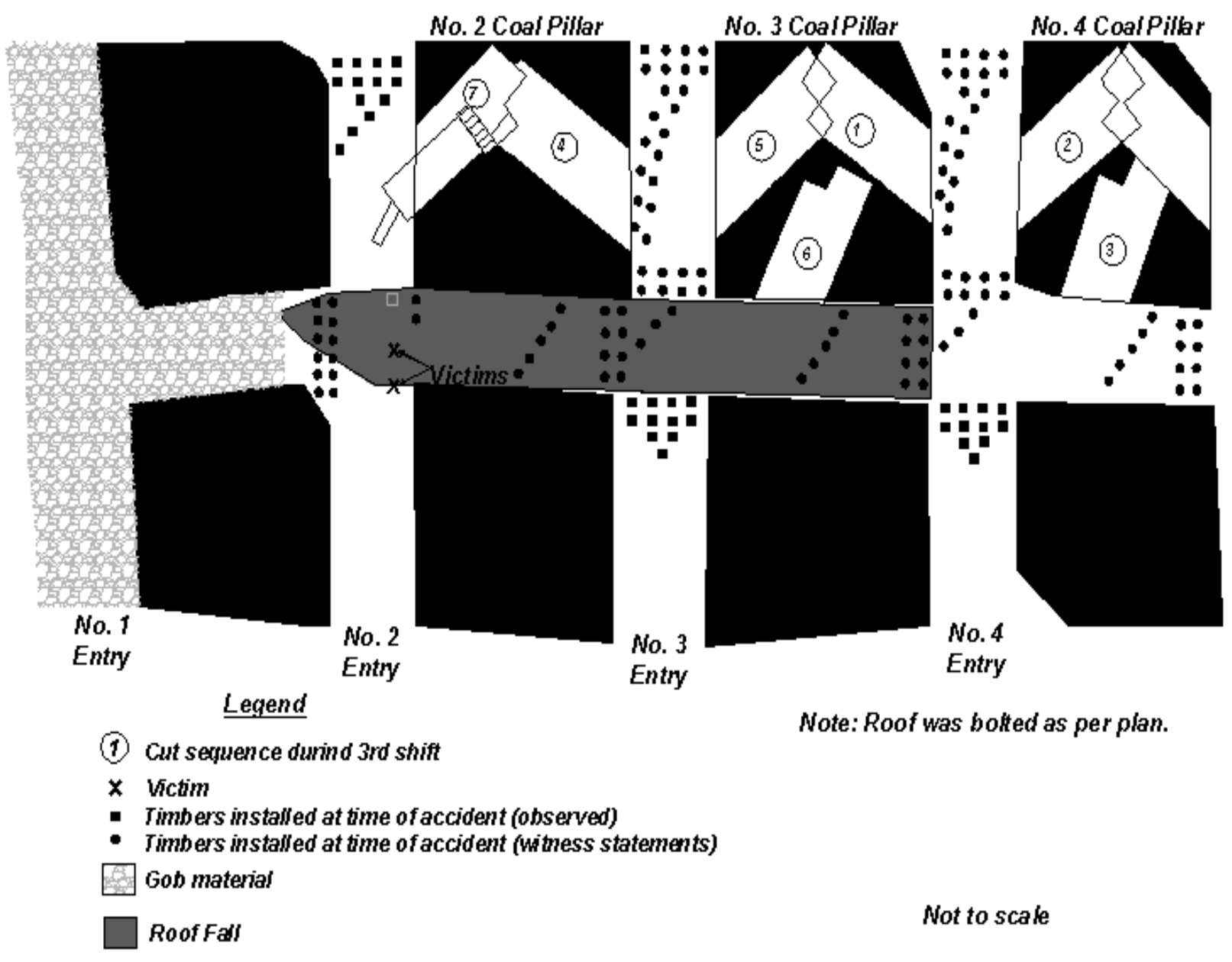

Figure 2.22. Roof Fall Fatality Drawing (MSHA, 2009) 


\section{Fatality Case Study No.2}

During the course of performing a risk assessment, time of exposure is an important factor. In this case study, a mobile-bridge operator was fatally injured by a roof fall while standing in the active mining zone. The reason for his presence there could not be determined by any of his coworkers; therefore, it can be assumed that he was simply observing from a favorable vantage point.

This case study has been selected because of the fatal injuries sustained by a miner whose presence was not required in the face area at the time of the incident and was, therefore, unnecessarily exposed to hazardous conditions. Exposure of nonessential personnel is an occurrence that was noticed repeatedly both during the evaluation of fatality reports and during mine-site observations (Discussed in Chapter 3 ). This case study also supports the hypothesis that MRS units may provide a false sense of security. As can be seen in Figure 2.23, the victim positioned himself between the No.1 and No. 3 MRS units prior to the fatal roof fall.

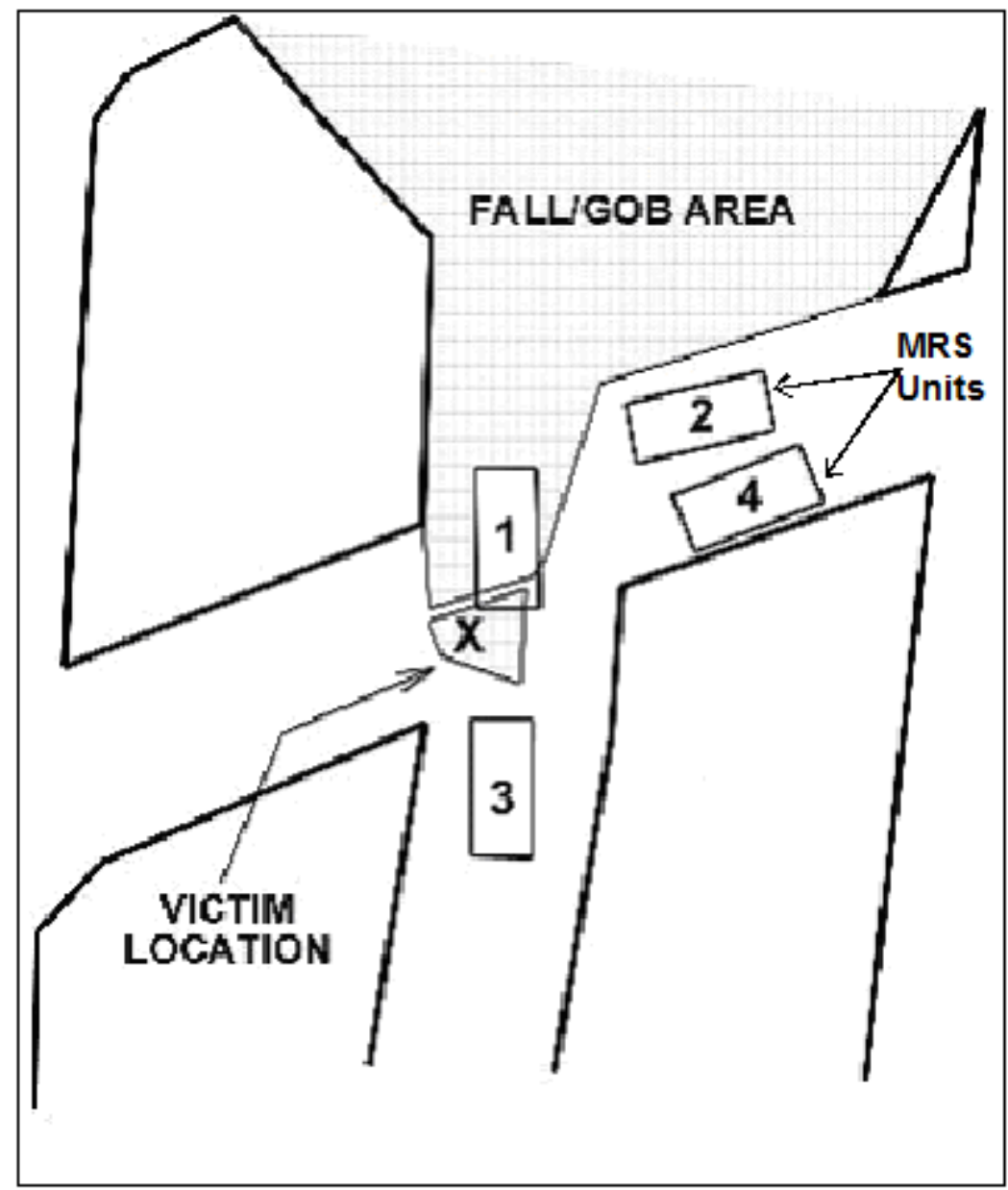

Figure 2.23. Roof Fall Fatality Drawing (MSHA, 2009) 


\section{Fatality Case Study No.3}

This case study has been selected because it supports the generally accepted theory that using solely timbers on a pillaring section increases the risk to miners working in the active mining zone. When compared to mobile roof supports, timbering is an antiquated method of roof support and, for the purpose of this thesis, it is believed that they should be used only as supplements to mobile roof supports. The comparably low load bearing nature of timbers, combined with relatively little yieldability or residual strength, makes timbers prone to being overrun when a fall propagates from the gob toward the active mining zone. The task of setting timbers is also very time consuming and usually requires the participation of numerous miners on the section and, therefore, significantly increases their amount of exposure in the active mining zone as well as increasing the number of material-handling injuries.

In this fatal incident a roof-bolter operator (functioning as a timberman) was killed by a roof fall, while observing roof conditions during the mining of the final cut on a pillar. As can be seen in Figure 2.24, the roof fall overran the turn posts that had been set during the mining of previous lifts and struck the victim. The victim was assigned to monitor the roof, making his presence in the active mining zone necessary, but his postion inby the miner may also have been a contributing factor.

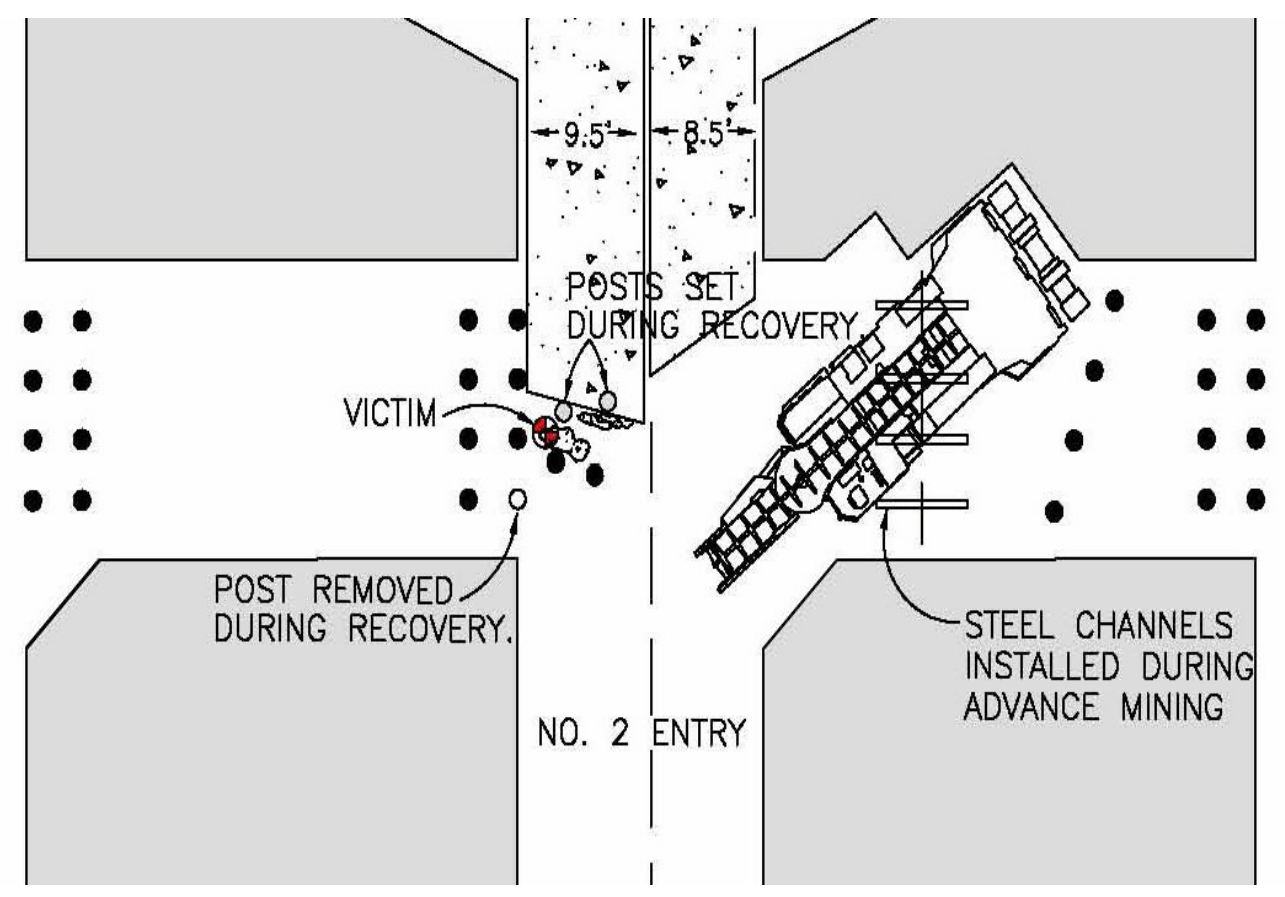

Figure 2.24. Roof Fall Fatality Drawing (MSHA, 2009) 


\section{Fatality Case Study No.4}

While it is generally accepted that mobile roof supports can significantly decrease the risk posed to miners on a retreating section, the setting or positioning of roof supports between cuts is a high-risk activity. Regardless as to whether or not the supports are timbers or mobile roof supports, and considering that the mobile roof supports are viewed to be far superior to timbers, MRS systems do not completely remove all risks. In some cases, the presence of mobile roof supports combined with a lack of training can cause a false sense of security. Miners feel far safer and, therefore, are comfortable behaving in a manner that they would otherwise deem to be unsafe. During mine-site observations (Described fully in Chapter 3.) the attitude that the MRS were capable of completely eliminating roof hazards was common, and operators seemed to have so much faith in the MRS units that they became complacent. Operating the MRS units from close range, as well as improper repositioning techniques, were commonly observed. These trends are mirrored in many of the fatality reports studied during the course of this thesis.

This particular incident was selected for further review, because it resulted in the deaths of two MRS operators, both of whom were operating the units outside of MSHA guidelines for safe operation (MSHA, 2005). The two victims were operating MRS units from a distance of only 15 feet when it appears that there was no obstruction or any other hindrance that prevented the operators from moving to a safer distance outby before depressurizing the units (Figure 2.25). This accident report also documents other violations of MSHA guidelines for MRS usage, such as the improper installation of breakaway cable hangers. 


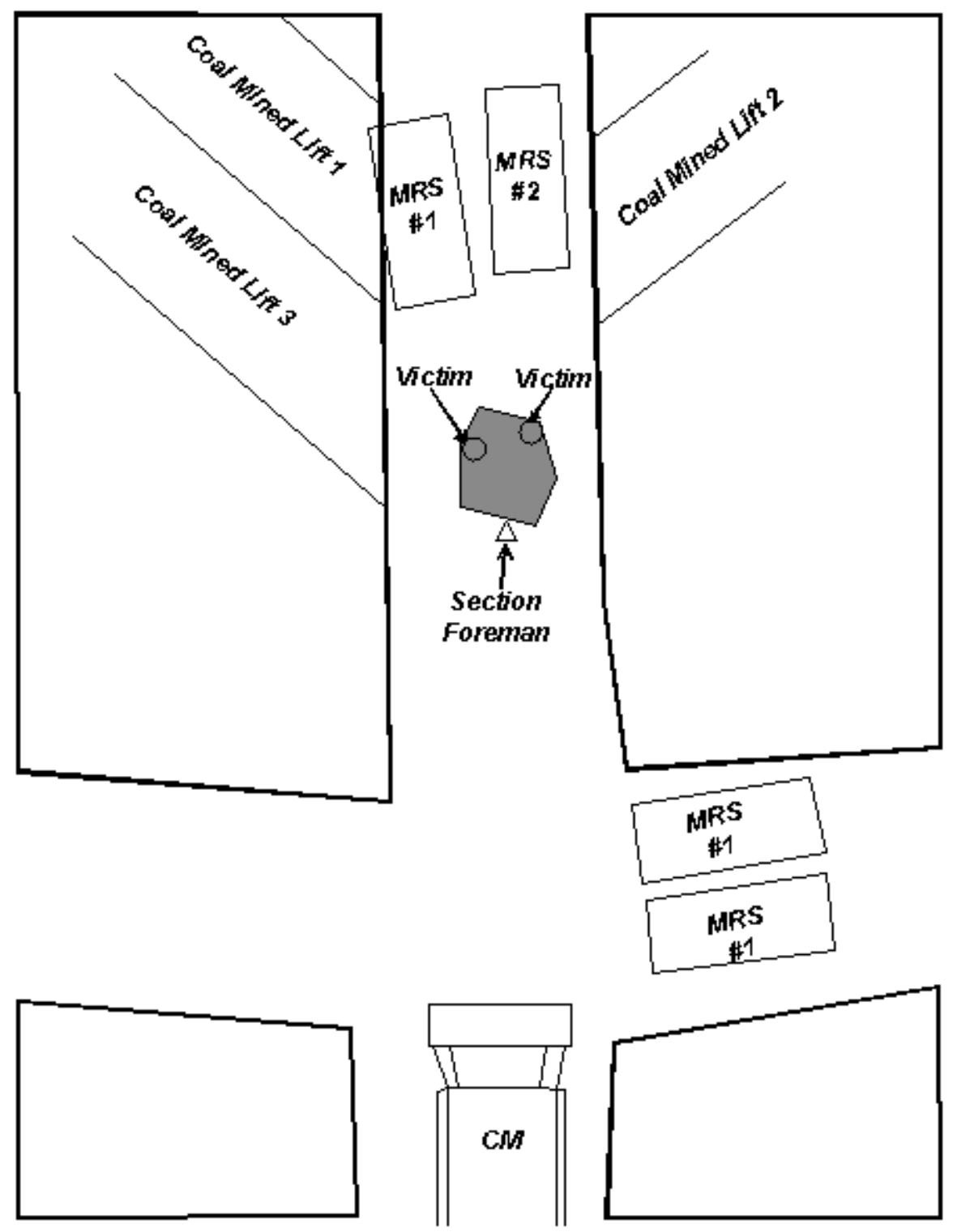

Figure 2.25. Roof Fall Fatality Drawing (MSHA, 2009) 


\section{Fatality Case Study No.5}

In the previous case study, operational concerns regarding the proper utilization of MRS units have been identified. Three of these concerns are very well represented in this incident; as a result, it has been selected for inclusion in this thesis. In this incident, there are three major MRS-related factors that may have significantly contributed to the fatalities: 1) mobile roof supports should be operated from a safe position at least 20 feet away, 2) mobile roof supports should never be repositioned more than one half of the unit length at a time, 3) manual controls on mobile roof supports are for maintenance purposes, only, and should never be used otherwise (MSHA, 2005).

While repositioning MRS units, one section foreman and one scoop operator, both functioning as MRS operators, were fatally injured by a roof fall. Both victims were located in close proximity to the MRS units, the manual controls were being used to operate the MRS unit immediately prior to the fall, and improper spacing of the MRS units during the repositioning may have contributed to the instability that resulted in the roof fall. The position of the victims and MRS units are shown in Figure 2.26.

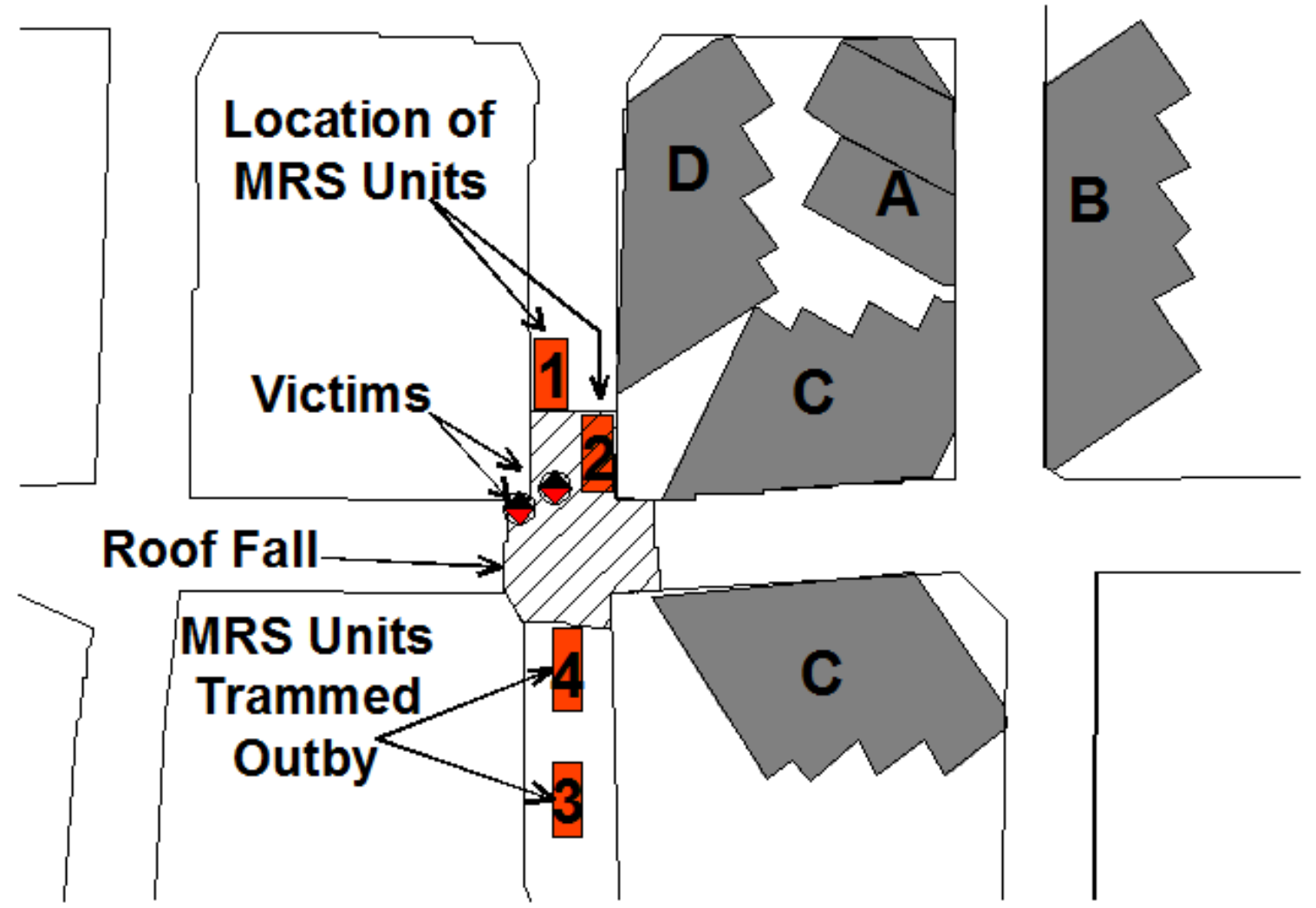

Figure 2.26. Roof Fall Fatality Drawing (MSHA, 2009) 


\section{Summary}

During the course of this literature review, every pillaring fatality (related to the fall of roof or rib) that occurred between 1995 and 2007 was carefully studied. In nearly every case, the previously discussed geotechnical risk factors played a significant role in the incidents. That was to be expected; however, the reason for reviewing the fatality reports was to view them from a miner-performance perspective, and ask the questions:

- How did the performance of the miners affect the outcome of the incident?

- What changes in performance could be made to possibly prevent the tragic outcomes of similar incidents if encountered in the future?

With these questions in mind, the following list of performance-based risk factors was developed based on the review of the fatality reports.

- Unnecessary exposure to geological hazards,

- Unnecessary personnel in hazardous areas,

- Improper positioning of equipment operator, or helper, and

- Failure to perform in accordance with MSHA guidelines.

In nearly every fatality report, if one of these performance-based risk factors had been reduced, the outcome may have been less severe, regardless of an inability to control the geotechnical risk factors.

\section{$\underline{\text { Risk Management }}$}

Risk management is a systematic and analytical process by which an organization identifies, reduces, and controls its potential risks and losses. Risk management not only evaluates assets, threats, and vulnerabilities but also incorporates a continuous analysis feature, which enables organizations to tailor their management of risk to the current situation, as well as to assess future risks (National Infrastructure Protection Center, 2002).

It is important to note that while the general methodology of risk management and safety management are very similar, the two terms are not interchangeable. Risk management is a broader term that refers to the management of multiple types of nonspeculative risk. The text by Bird and Germain (1990) describes risks to people, property, or process. These types of risk are non-speculative due to the fact that the only possible outcome of an incident is a loss. There is no possible gain to be made by accepting these risks, therefore, reducing them can only prevent loss and benefit efficiency.

While the majority of risk-management texts are in agreement with regard to the major concepts of risk management, the terminology varies widely. The following table depicts risk management structure described by various texts and authors. 
Table 2.5. Examples of Risk Management Structure

\begin{tabular}{|c|c|c|}
\hline Brauer (2005) & Petts (1998) & FEMA (1993) \\
\hline 1. Risk identification & 1. Hazard identification & 1. Establish a planning \\
\hline 2. Risk analysis & 2. Hazard analysis & team \\
\hline 3. Financing risk & 3. Risk estimation & 2. Analyze capabilities \\
\hline 4. Administering the & 4. Risk evaluation & and hazards \\
\hline risk management & 5. Implementation & 3. Develop the plan \\
\hline process & $\begin{array}{l}\text { 6. Monitoring and } \\
\text { auditing }\end{array}$ & 4. Implement the plan \\
\hline
\end{tabular}

As shown in Table 2.5, risk management structure lacks consistent terminology. This terminology difference is seen between various authors as well as between different specialized usages of risk management. However, when risk management processes from various authors, industries, and agencies are reviewed, most share the following characteristics (although they differ in terminology): 1) hazard identification, 2) risk analysis, 3) development of risk controls, 4) implementation of controls and, 5) monitoring the effectiveness of controls. It is this basic structure, upon which industries and agencies customize risk management strategies to meet their individual needs. Therefore, these same five steps will serve as the basis of the risk management strategy that will be developed to address the unique risks associated with pillar extraction.

\section{Hazard Identification}

Covello and Merkhofer (1993) define hazard identification simply as the process of identifying new sources of risk. Bise, et al. (1993) identifies three types of hazards:

1. Worker performance-related hazards,

2. Direct hazards,

3. Indirect hazards.

Each hazard type is then subdivided into categories, and then individual hazards are identified within each category. For example, hazards could be described as safety hazards, health hazards, environmental hazards, public welfare hazards or financial hazards, but they would still fall under one of the three previous categories.

For this thesis, direct and indirect hazards will be identified but, due to extensive attention paid to these hazards in previous research, the goal of this thesis is to identify worker-performance-related hazards and their interactions with direct and indirect hazards. 


\section{$\underline{\text { Risk Analysis }}$}

When conducting a risk analysis, it is important to emphasize the relationship between a hazard, and a risk. In this thesis, a hazard is considered to be the source of risk and remains constant; the risk associated with a hazard varies due to consequence and probability. Factors that affect the probability of a risk will be referred to as risk factors.

According to Bilal (2005), there are two categories of risk analysis: quantitative, and qualitative. Quantitative analysis relies on probabilistic and statistical methods, and databases that identify probability values and consequence values for risk analysis. This approach is objective and examines the system in greater detail to assess risks. Quantitative analysis generally provides a more uniform understanding among different individuals, but requires quality data for accurate results, (Bilal, 2005). Qualitative analysis is a subjective method that relies on the judgment and opinions of experts to assign risk and probability. Qualitative methods incorporate analyses without detailed information, but the intuitive and subjective processes may result in differences in outcomes based on the experience and expertise of those that use them (Bilal, 2005). Due to the significance of expertise on the accuracy of qualitative analysis, it is important that personnel charged with performing risk analysis are knowledgeable in the areas being analyzed.

As stated in the previous paragraph, analysis methods are generally classified as quantitative or qualitative but, in practice, the dividing line between the two classifications is blurred. Most practical methods of analyzing risk utilize as much data as is available to help influence judgment, thereby combining qualitative and quantitative risk analysis. In the mining industry, accurate statistical information may not always be available for every situation, typically necessitating the integration of qualitative and quantitative analysis methods to accurately assess risk. This is the case in pillar extraction. The resulting risk analysis strategy will be developed to maximize quantitative information in order to provide for more accurate qualitative assessment and improved risk controls.

There are many types of risk-analysis methods utilized by government and industries, and each have their own individual strengths and weaknesses. Therefore, in order to maximize accuracy, most risk management strategies utilize multiple analysis methods that are applicable to the specific need of the industry or organization. The following sections summarize several forms of risk analysis that are well suited for mining applications.

Monitoring. Monitoring is most often used in cases of environmental risk, such as pollution or contamination. In these instances, the pollutant or contaminant build up (hazard consequence) usually occurs over an extended period of time and can be termed "slow release" when compared to something like an industrial explosion. It is this gradual change in the system that makes monitoring a very useful tool. By monitoring the system, as well as similar systems that lack the hazard exposure, a baseline for comparison can be made and, as a result of the baseline comparison and rate of progression, the risk can be estimated. As previously stated, monitoring works 
well when the hazard release is slow, but will prove to be of little use when the hazard release is rapid (Covello and Merkhofer, 1993). In the case of monitoring, there is usually a set level at which hazard release/exposure becomes unacceptable, so while exposure remains below an acceptable limit, there is no loss, making monitoring a preloss analysis.

Statistical Analysis. The underlying assumption in statistical methods is that a statistical model can be used to describe the outcomes of situations that occur repeatedly in the real world. Such models assume that events occur randomly, but that something is known about the mathematical character of the processes that produce the events (Covello and Merkhofes, 1993).

When the exposure-consequence relationship is simple, such as when nearly every exposure results in death, then the risk of a fatality is easily assigned based upon the probability of exposure. This is why statistical analysis is particularly good for analyzing hazards that occur frequently and have well documented consequence data. Car wrecks are a good example. This type of analysis is often referred to as a historical risk analysis and, since it only deals with the data related to hazard consequence, it rarely sheds any light on the risk factors that contribute to the hazard. So while statistical analysis is an excellent tool for directly assessing risk, it should not be used exclusively; rather, it should be integrated with other analysis methods.

Risk Modeling. For discrete risks such as accidents, risk-source modeling is a very effective method to assign probability and identify characteristics of an accident without the accident ever having to take place. However, in cases where an event has occurred, the risk source may be modeled according to the particular event. There are several types of risk modeling that are commonly practiced. The two that will be discussed are Failure-Mode and Effects Analysis (FMEA), and Fault/Event Tree Modeling.

Failure Mode and Effects Analysis. Failure-Mode and Effects Analysis (FMEA) consists of reliability information, functional diagrams, and coding that identify system components. FMEA models look at the components of the hazard, the circumstances surrounding the hazard, and the sequence of events that must occur before an accident or failure can take place. By identifying the components and their relationships to one another, as well as the component reliability, failure scenarios can be developed (Covello and Merkhofes, 1993).

Once the FMEA has been completed, a fault hazard analysis (FHA) can be performed. The FHA determines the impact of failure scenarios on other system components in order to gain a better understanding of total system effects and any possible additional hazards caused by the failure scenarios, (Covello and Merkhofes, 1993).

Once the FMEA and FHA have been completed, a criticality analysis (CA) can be performed. The CA studies the system and determines the relative magnitude of each failure scenario based on probability and effect on the system. It is in this step that risk is assigned based on the probability and consequence of the failure modes, as defined by the previous models (Covello and Merkhofes, 1993). 
Fault/Event-Tree Modeling. According to Covello and Merkhofes (1993), this method is the most frequently used for creating a model of a discrete risk source. In the previously described modeling technique, hypothetical post-loss scenarios were analyzed to determine the impact on a system related to a particular hazard/event. In a fault- or event-tree analysis, the thinking is the opposite. A fault- or event-tree analysis is a pre-loss model that begins with an event or accident and looks backwards to determine what scenario of events had to take place for the accident to occur. By isolating precursors, it is possible to identify an increased risk when it is encountered in the future. In order to isolate precursors, a fault or event tree is usually set up as a diagram of questions with yes/no or pass/fail answers that are intended to highlight the event scenario. This type of modeling is termed "Root Cause Analysis" by MSHA and is utilized during accident investigations. Covello and Merkhofes (1993) states that this type of risk modeling is complementary to other types of modeling, in that the consequence scenarios developed in a model, such as a FMEA, are combined with precursor scenarios developed with the event-tree model, the risk managers are provided pre-loss and post-loss information relating to a hazard and are better equipped to assign risk to that hazard.

Performance Observations. While differing in some terminology, well known safety management-based texts such as Brauer (2005) and Della-Giustina (2000), stress the need for performance observation to accurately assess a job/task. Brauer (2005) states that, an unsafe condition or an unsafe act is rarely the sole cause of an incident but, that causation usually involves the interaction of both. Previously discussed analysis methods are well equipped to identify unsafe conditions, but do not thoroughly document unsafe acts or poor performance. In the case of event tree or failure mode and effects analysis, it is possible to speculate on what unsafe acts may occur, but there is no actual observation of performance that may have lead up to the speculated unsafe act. The information gathered by performance observations accounts for the other side of the analysis equation, that other methods are not equipped to cover. In the text by Bird and Germain (1990), planned observation is described as an organized and systematic tool to assess how well people are performing specific jobs or tasks. Through planned observation, substandard practices are likely to be identifed and corrected before potential losses become actual loss. Planned observation is a way to assess employee knowledge of proper job performance (Bird and Germain, 1990). In this thesis, the objective of performance observation is to obtain quantitative information that will provide managers and trainers with the necessary information to control risk through training or other types of intervention. This feedback yields valuable information regarding the effectiveness of training programs and job instruction, and the adequacy of existing job/task procedures (Bird and Germain, 1990)

By combining applicable analysis methods to identify unsafe conditions with performance observations that identify unsafe acts or improper job/task performance, it is possible to provide management and trainers with a more complete picture of the overall risk, making them better equipped to develop effective methods to control risk. 


\section{$\underline{\text { Risk Control }}$}

Upon completion of a risk analysis, the planning team should have an in-depth understanding of the risks associated with the evaluated hazards. Based on this understanding, the planning team is then charged with the task of prioritizing hazards for risk control, first addressing high risk hazards and progressing to hazards of lower risk.

The following is a list of controls as described by the Air Land Sea Application Center (2001):

- Engineering Controls- These controls use engineering methods to reduce risks, such as developing new technologies or design features, selecting better materials, identifying suitable materials or equipment, or adapting new technologies to existing systems.

- Administrative Controls- These controls involve administrative actions such as establishing written policies, programs, instructions, and standard operating procedures, or limiting the exposure to a threat either by reducing the number of personnel/assets or length of time they are exposed.

- Educational Controls- These controls are based on the knowledge and skills of units and individuals. Effective control is implemented through individual and collective training that ensures performance to standard.

- Physical Controls- These controls may take the form of barriers and guards or signs to warn individuals and units that a threat exists. Use of personal protective equipment is a good example.

- Operational Controls- These controls involve operational actions such as controlling areas of operation, boundaries, scenario rehearsals, workforce management and supply-chain management.

Even though there are several methods of controlling risk, the focus of this thesis on pillaring safety is geared towards the use of administrative, educational, and operational controls, due to the continued impact of residual risk resulting from the shortcomings of engineering and physical controls. This residual risk is evident by continued injuries and fatalities on pillaring sections, necessitating control by other means, such as education and other types of preventative intervention.

\section{Policy Implementation}

After risk controls have been developed, management is tasked with selecting and implementing controls. Each control has a cost and residual amount of risk associated with it. Management must decide on an acceptable level of residual risk and 
then assign the necessary resources needed to implement the control (Brauer, 2005). No matter how potentially effective risk controls may be, they will not benefit an organization unless they are properly implemented. In many cases, risk controls result in changes in the way that a job may be performed. These changes may be met with resistance by a workforce that is accustomed to performing tasks in a particular manner. In these cases, simply suggesting control criteria is not sufficient. It is important that those charged with managing risk have the authority or backing from upper management to implement and enforce risk controls. In addition to having proper authority, it is critical to have the understanding and cooperation of personnel supervisors in order to effectively implement controls on every operational level.

\section{Monitoring}

Monitoring is a part of risk management that is too frequently overlooked, because most industries tend to be reactive rather than proactive. The text by Brauer (2005) states that:

Administering a process necessitates monitoring and evaluating if reductions were achieved, if frequency and severity actually resulted as projected, and if expenditures achieve the benefits that were anticipated.

In the case of operational, educational and administrative controls, it is particularly important to monitor performance following the implementation of a policy in order to ensure effectiveness of the policies. By monitoring the effectiveness of risk controls, through physical observation, record keeping of accident trends, or soliciting employee input, management can judge the effectiveness of control policies. If the policies prove to be effective, risk managers should continue to monitor the hazard but, should also re-focus attention to the hazard the poses the next highest risk. If risk controls are failing, then they must be re-evaluated before moving on to the next hazard. Monitoring may be accomplished by incorporating some of the analysis methods used in the initial risk assessment. Utilizing statistical analysis to follow incident trends, combined with periodic performance observation is an effective method to evaluate the impact of risk controls and control implementation. 


\section{Chapter Summary}

As long as coal has been mined in Central Appalachia, pillaring has been practiced. Since the days of hand-loading, pillaring techniques have undergone a drastic evolution. The implementation of remote-controlled continuous miners, as well as MRS units, represents the modern stage of this evolution. There are many geotechnical risk factors related to pillaring such as:

- Cut sequence,

- Final stump,

- MRS vs. timbers,

- Roof bolting,

- Geology,
- Pillar age and uniformity, and

- Continuous haulage, as it affects entry and pillar design

Engineers and mine managers attempt to control these risk factors through design, advanced machinery and modern roof control strategies. Even though these controls typically represent the best engineering knowledge, modern materials, and machinery, they fail to control all of the risks related to pillaring. The presence of residual risk is evident by the 34 pillaring-related fatalities that occurred between 1995 and 2007. Residual risk is certainly present in Central Appalachia, since $76 \%$ of the fatalities occurred here. With the high level of modernization that is present on pillaring sections, the following question needs to be addressed: Why are fatalities still occurring?

Initial assumptions might be that the most modern equipment is not being utilized, or that modern equipment or methods were not being utilized properly. Since no instances of any mine extracting pillars without the use of a remote-controlled continuous miner was encountered, it was possible to focus attention to the MRS, since it is not utilized at every operation due to cost and operational height constraints.

Upon focusing attention on MRS-related fatalities, it was determined that $62 \%$ of the 34 pillaring-related fatalities that occurred between 1995 and 2007 occurred on sections that did not utilized MRS units. The remaining fatalities occurred where MRS units are utilized on the section. From this evaluation, it became apparent that the MRS likely does improve safety, but the continued occurrence of fatalities indicates the possibility of improper job performance on the section and/or limitations of the MRS to completely control roof falls.

In order to better understand the performance-related factors that contributed to pillaring fatalities, it was important to not only compile statistical information, but also to study the fatality reports, as well. Although every fatality between 1995 and 2007 was studied, only five were presented in this thesis. These five best represented the accident trends that were seen throughout the fatality review. The review of fatality reports confirmed assumptions made during the statistical review that MRS units do reduce, but do not eliminate the risk of roof falls and that mineworker performance plays a major role in accidents. Although instances of falls in the gob, propagating into the active mining zone were reduced by the usage of MRS units, falls still occurred, both propagating from the gob, and initiating in the active mining zone, when MRS units were used. In most cases, the accidents and the severity of the outcome of the accidents could have been drastically altered if mineworker performance had been in accordance 
with recommended standards and procedures. While each accident was unique, there were re-occurring performance issues that significantly impacted the severity of the accidents, such as;

- unnecessary exposure to geological hazards,

- unnecessary personnel in hazardous areas,

- improper positioning of equipment operator, or helper, and

- failure to perform in accordance with MSHA guidelines.

Based on the review of geotechnical-based pillaring research, as well as the reviews of fatality reports, it can be concluded that the safety and success of a pillaring operations is heavily influenced by both geotechnical and mineworker-performance factors.

Since the goal of this research was to develop a risk-management-based strategy to improve pillaring-related safety, it was important to review numerous techniques for managing risk. The risk-management techniques most applicable to pillar extraction were presented in this chapter. In Chapter 4, these techniques will be modified to meet the unique risks posed by the work environment on a pillaring section. The flowchart shown in Figure 2.27 represents the general risk-management strategy based upon the information presented in this chapter. 


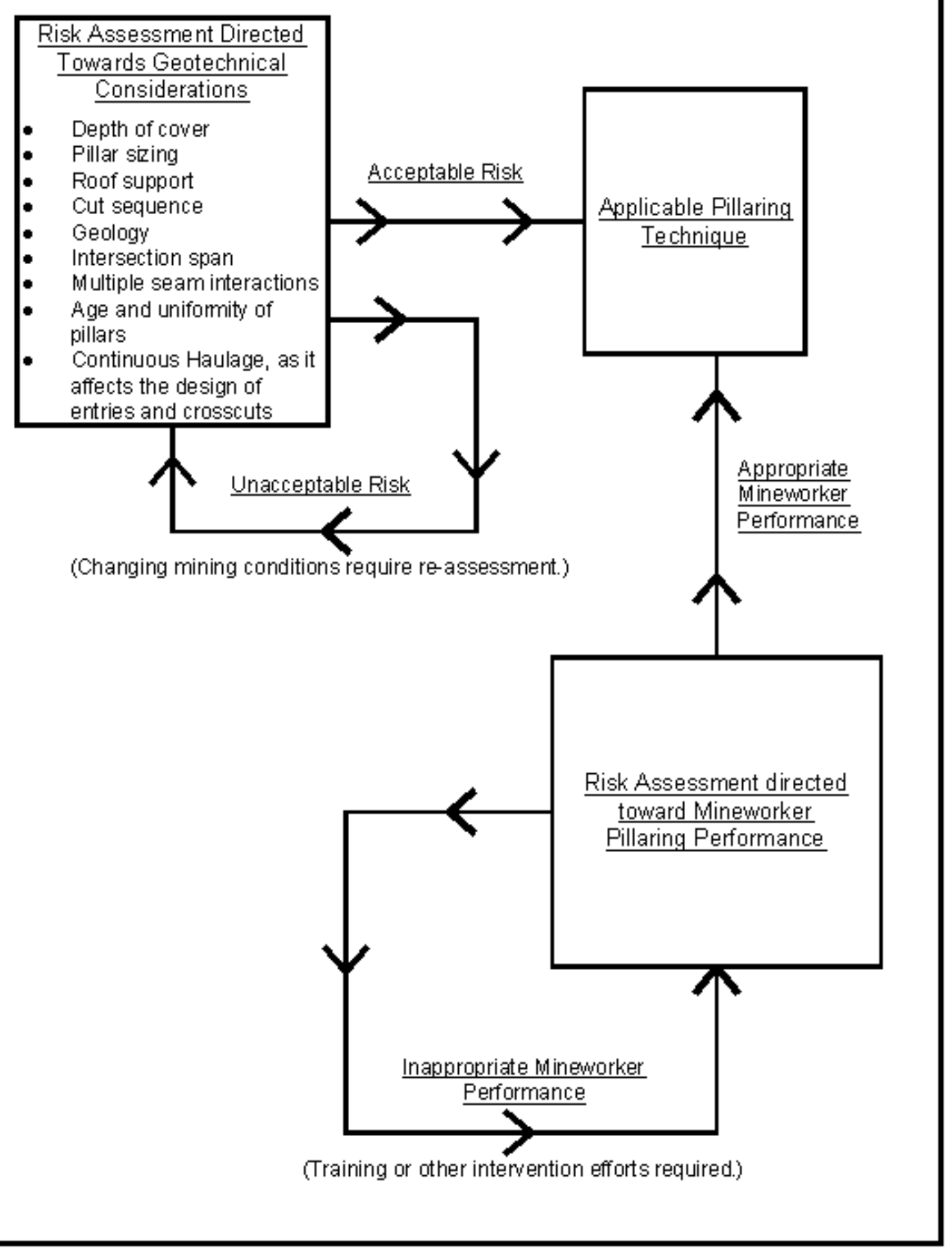

Figure 2.27. Risk Management Flowchart for Pillaring (Bise, 2007)

The strategy outlined in Figure 2.27 is a two-pronged attack on risk, with the ultimate goal being an applicable pillaring technique. The attack is two pronged because it accounts for geotechnical-based risk and performance-based risk, both of which must be satisfactorily addressed. 
Geotechnical-based risk factors must be controlled through engineering and physical methods. By evaluating engineering design and physical controls, it is possible to achieve an acceptable level of risk. If design criteria or physical controls do not adequately control geotechnical risks, the control development process must continue to cycle until satisfactory results are achieved.

Control of performance-based risk requires a different strategy. In the case of performance-based risk, it is widely accepted that training and preventative intervention are the most effective means of minimizing poor performance. However, developing effective training is more complex than it is often perceived. In order to develop an effective training strategy, it is necessary to evaluate the current educational needs of the workforce. In order to gauge the effectiveness of a training program and employee compliance with training, it is necessary to know what the employees are doing in the work environment. While compiling accident statistics and reviewing accidents reports can and should be utilized in the development of safety training, it is important to recognize that this is a reactive approach to safety. If a more proactive approach to training is taken there will likely be less accident information to review. In order to learn from the past, and take a proactive stance to safety training, it is necessary to integrate historical information with predicted safety concerns. This proactive approach can be accomplished by utilizing structured performance observations to assess training needs and evaluate effectiveness of training strategies.

By developing a comprehensive risk management strategy that employs engineering controls to combat geotechnical risk factors and emphasizes training and performance evaluation in order to combat performance based risk, it should be possible to significantly improve the safety and applicability of pillaring. 


\section{CHAPTER 3. FIELD OBSERVATIONS}

\section{Introduction}

As previously discussed, modern pillar extraction utilizes two main pieces of equipment: 1) the continuous miner and 2) mobile roof supports. While there are several variations of continuous miners available for thin-seam extraction, current mobile roof support technology is limited to a seam thickness above 42 inches (Burgess, 2009). Although no recent studies pertaining to the percentage of reserves recoverable by means of pillar extraction are available, a study performed by the United States Geological Survey approximately 50 years ago categorized coal reserves in Central Appalachia by seam thickness. Based on that study, and the working height of current mobile roof supports, it was estimated that approximately 50 billion tons of reserves in that previous study were in excess of 42 inches and recoverable by pillar extraction. Due to the age of this reserve estimation, it should be assumed that many of those reserves have been mined. However, this study is useful because it demonstrated that, at the time, approximately $50 \%$ of the estimated reserves were located in seams in excess of 42 inches and thereby recoverable with modern mining techniques utilizing MRS. After contacting representatives from the West Virginia Geological and Economic Survey (Blake, 2010), Kentucky Geological Survey (Weisenfluh, 2010), and the Virginia Department of Mines, Minerals and Energy (Lassettier ,2010), it has become apparent that current estimations of Central Appalachian reserves in excess of 42 inches do not currently exist. So while there is not current information available with regard to the seam height of current Central Appalachian reserves, there are current reserve estimations for Central Appalachia that encompass all seam thicknesses. For the purpose of this thesis, it will be assumed that the same percentage of MRS recoverable reserves - - - 50\%- - - is applicable to today's estimation of reserves, even though there have been numerous technological advancements over the past few decades that may raise the percentage of recoverable reserves. So based on this $50 \%$ estimation and the United States Energy Information Administration's 33 billion ton reserve estimation for West Virginia, Virginia and Kentucky, it can be conservatively estimated that Central Appalachia has well over 15 billion tons of reserves in which pillar extraction is applicable (USEIA, 2007). These numbers assure pillar extraction's future consideration in the Central Appalachian coal industry, and makes it a crucial for extracting as much of the reserve base as possible.

\section{Mine Site Descriptions}

Through the literature review conducted in this thesis, it has been possible to determine the general operational aspects affecting successful pillar extraction. The first aspect affecting the ability to extract pillars is strata control, while the second is employee safety and training, and the third is economics. While, in many cases, it may be physically possible to extract pillars, it may not be safely accomplished without a properly trained and properly motivated workforce. 
During the extensive review of pillar-related fatalities presented in Chapter 2, it became evident that even though many of the accidents had contributing factors related to strata instabilities, many, if not most, fatalities also involved mineworker performance factors that could have equally affected the outcome of the incident. It can be assumed that mineworker performance plays a similar role in non-fatal accidents, as well. With this in mind, the mine visits discussed in this chapter were scheduled to observe workplace conditions and miner performance of job activities and to determine if current performance still bears similarities to the performance noted in fatality investigations. By visiting a mine site and observing the worksite performance of miners on an active section, it was possible to get a better understanding of how performance and safety training impact successful extraction of pillars. In addition, it was possible to identify actions that may have a negative impact on safety, as well as new risk factors that may have previously been overlooked. All mines visited were located in West Virginia; however, names and exact locations will not be included for confidentiality purposes.

\section{$\underline{\text { Mine A }}$}

Mine A was located in southern West Virginia. It was extracting pillars with a mining height ranging from nine to eleven feet. The immediate roof consists of approximately 8 feet of massive sandstone, while the floor was predominately fireclay. The section was under approximately 400 feet of cover. Overmining and undermining of the active seam have both occurred with 80 and 90 feet of innerburden, respectively. The fireclay floor proved to be problematic because it did not facilitate the drainage of groundwater through the mine. Water and thick mud accumulation in the floor was often as deep as 10 inches, and required the construction of sumps, as well as constant pumping. Entry spans were designed at 18.5 feet and pillars were sized at 80 by 52 feet. Crosscut angles were driven at 90 degrees. The active section was three months old and pillars showed little sign of degradation. The roof bolting consisted of 6 - $\mathrm{ft}$ torque-tensioned bolts on a 4-bolt pattern across the entry with approximately 4 feet between rows. In places where irregularities in the roof were encountered, steel straps were used in conjunction with the bolts. 


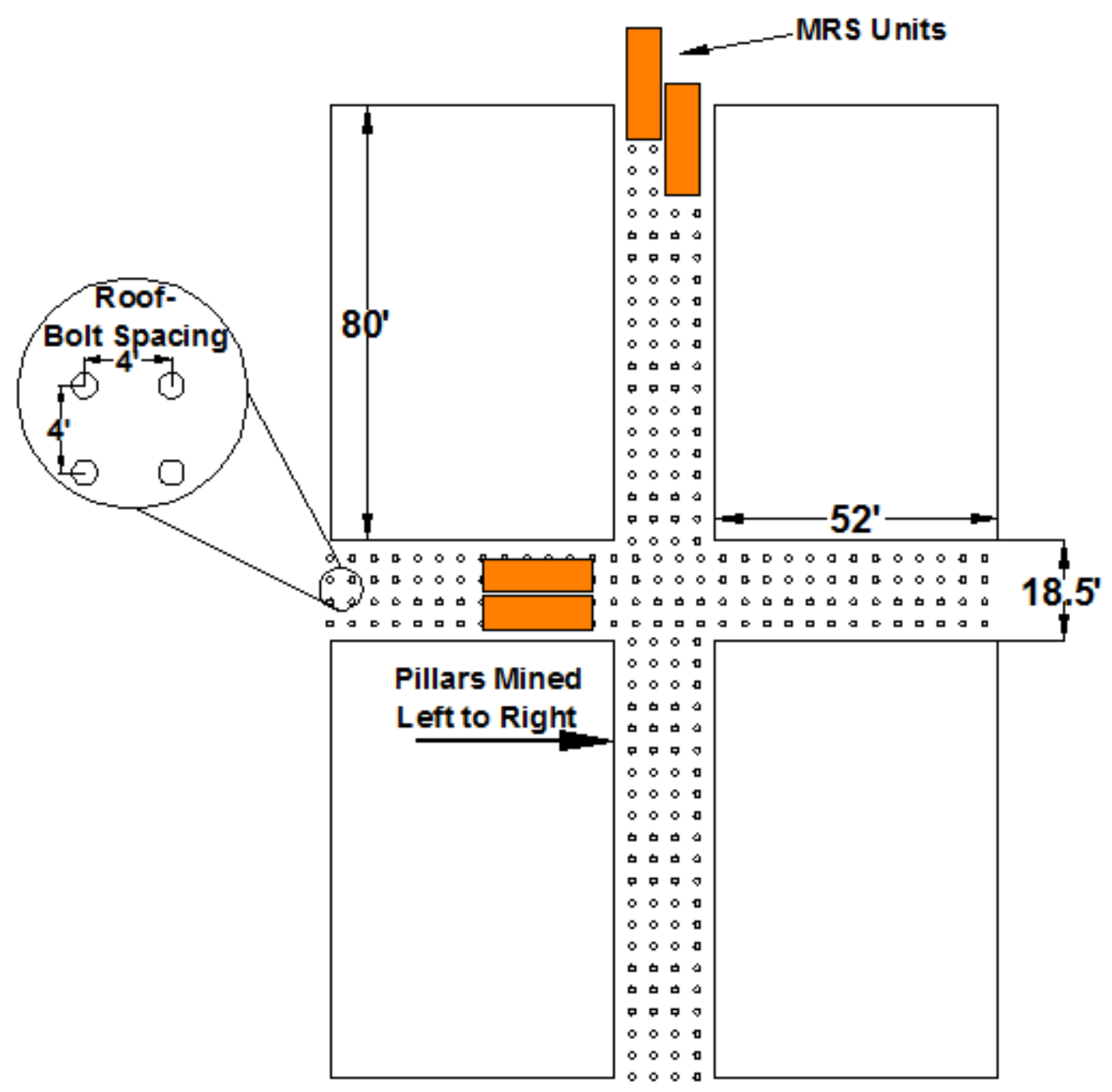

Figure 3.1. Mine A Observed Dimensions and Roof-Bolting Pattern

Pillars were extracted in the section from left to right, utilizing the Christmas-tree cut sequence with an approximate cut depth of 26 feet. Two sets of Fletcher 800-ton mobile roof supports were utilized in conjunction with the setting of breaker posts in the next crosscut outby prior to a lateral move to the next pillar. The coal was extracted using a remotely operated Joy $12 \mathrm{CM} 27$ continuous miner. Coal was hauled using two cable-reel shuttle cars and one battery-powered car. The mine operated two daily productions shifts and one daily maintenance shift for six days per week. A ten-man crew operated on the section; the crew consisted of two mobile roof support operators, an electrician, a scoop operator, two cable-reel shuttle car operators, a battery-powered coal hauler operator, a continuous miner operator, a continuous miner helper, and a foreman.

General Observations. The working area was kept clean of debris. At no point was a miner observed moving through a pinch point, while equipment was being operated or trammed. One area of concern to be noted was that the section foreman did not appear to be focused on the activity at the working face. For the majority of the cut sequence, 
the foreman sat on a bucket in the last open crosscut with his back to the face, talking about unrelated issues with the MRS operators. At one point the foreman was engaged in horseplay, which resulted in the participants coming in close proximity to a shuttle car as it passed. The violation of the MSHA roof control plan by the use of pine timbers as opposed to hardwood timbers (hardwood required at this mine) as well as improper handling of the high-voltage miner cable were noted. The last area of concern involved the MRS operators and their position during downtime. Both MRS operators positioned themselves against the two unpressurized MRS units, located in the first outby crosscut, while they were not performing tasks. While there were areas of concern, the crew did communicate well. The shuttle car operators, as well as the battery-powered coal hauler operator, did a good job of sounding an audible alarm before proceeding through check curtains.

MRS Observations. The retreating section employed two miners with the job titles of Mobile Roof Support Operators. Neither operator was able to provide an exact answer as to how long they had been in that position but indicated that it had been longer than three years for both men. They described their MRS training as task training taught by the section foreman. While not operating the mobile roof supports, the MRS operators sat in the first crosscut outby the pillars being mined, directly in front of the two mobile roof supports positioned to the left of the first outby crosscut. It was observed that neither of the two MRS units positioned in the crosscut was pressurized against the roof. When questioned about the lack of pressurization, the response from the operators was that due to the local conditions it was not necessary to pressurize the units at that time. Operators were informed about the yielding pressure at which operations are to cease and personnel are to be moved out (4350 psi according to the roof control plan), but when asked who is the person responsible for monitoring the MRS gauges during the cut, there was some debate before it was agreed that the responsibility fell to the Continuous Miner Helper. The setting pressure of the supports was to be between 1500-2000 psi, and was displayed on 6-in. dial gauges.

According to MSHA's Program Information Bulletin PO5-19 for the use of mobile roof supports, there were several areas of concern that were identified during the course of this observation. These areas of concern are listed and described as follows.

- $\quad$ Onboard, manually-operated controls should be "locked out" or under a "bolted down" cover plate to restrict their usage to maintenance and troubleshooting purposes only (MSHA 2005).

When the foreman was questioned as to whether or not the manual controls were secured in the aforementioned required manner, he stated that some were and some were not, but that they were only used for maintenance purposes.

- $\quad$ When setting and lowering the MRS, the operator should be positioned in a safe location, at least 20 feet away from the units (MSHA 2005). During repositioning of MRS units, operators were observed standing as close as $15 f e e t$ to the units they were operating. 
- $\quad$ When moving the mobile roof supports from pillar lift to pillar lift, each MRS should be advanced sequentially such that one unit will never be offset more than one half the length of its companion unit. The MRS units should be advanced immediately after each lift, and should be kept as close as practical to the continuous mining machine during each lift. Upon completion of mining in a given pillar, the MRS units should be moved sequentially until they are between solid coal pillars. During this process, at least one unit will be pressurized against the roof at all times (MSHA 2005).

MRS units, inby the miner, were observed being moved in excess of one unit length during repositioning between cuts. In one instance, both inby units were sequentially depressurized without the re-pressurization of the first unit, and for nearly two minutes, neither unit was in contact with the roof.

- Install the MRS cables with break-away cable hangers so that the cable can be pulled down remotely (MSHA 2005). During repositioning of the inby MRS units, over tightening of the break-away hanger wires resulted in personnel going within 10 feet of unpressurized MRS units to take down the hanging cable.

Summary. The miners on this particular section, were either not familiar with the MSHA recommendations for the use of mobile roof supports, or were disregarding these recommendations. Based on the observations, it is perceived that the use of the MRS units on the section made miners more comfortable and possibly complacent, causing them to take unnecessary risks that they might otherwise avoid. It is these lapses of judgment that are recurrent in pillaring-related fatality reports.

\section{$\underline{\text { Mine B }}$}

Mine B was located in southern West Virginia and extracted coal from a seam with a mining height which varied between 40 and 60 inches. The maximum depth of cover was $1000 \mathrm{ft}$ and, although no undermining had occurred, four seams had been overmined, the closest located two hundred feet above the seam containing Mine B. The immediate roof consisted of 10 to 18 inches of shale while the main roof consisted of sandstone approximately $20 \mathrm{ft}$ thick. The floor was a fireclay-and-shale mixture. Entry and crosscut widths were $20 \mathrm{ft}$. Average pillar dimension was $50 \mathrm{ft}$ by $70 \mathrm{ft}$, with the 70 $\mathrm{ft}$ side being parallel to the entry. Pillars were mined using a Joy $14 \mathrm{CM}-15$ continuous miner. Two Voest-Alpine MRS units, combined with timbers, were utilized for roof control on the retreating section. Coal was transported from the section using two Joy 21 SC shuttle cars. An S\&S scoop delivers materials to the section, and was used for performing miscellaneous housekeeping tasks. Mine B was no longer being developed and the production was solely from pillar extraction. During the year of the on-site observation, Mine B employed 55 people and produced 270,485 tons of coal. 60 -inch fully grouted roof bolts spaced on 4 -ft centers were the primary method of roof support. Supplemental supports, such as steel straps, cable bolts, and wood cribs, were also utilized. Two visits were made to Mine B to observe the extraction of pillars; the 
Christmas-tree (Figure 3.2) and Outside Lift (Figure 3.3) cut sequences were both utilized.

Full Pillar Recovery

Using Posts and Mobile Roof Support

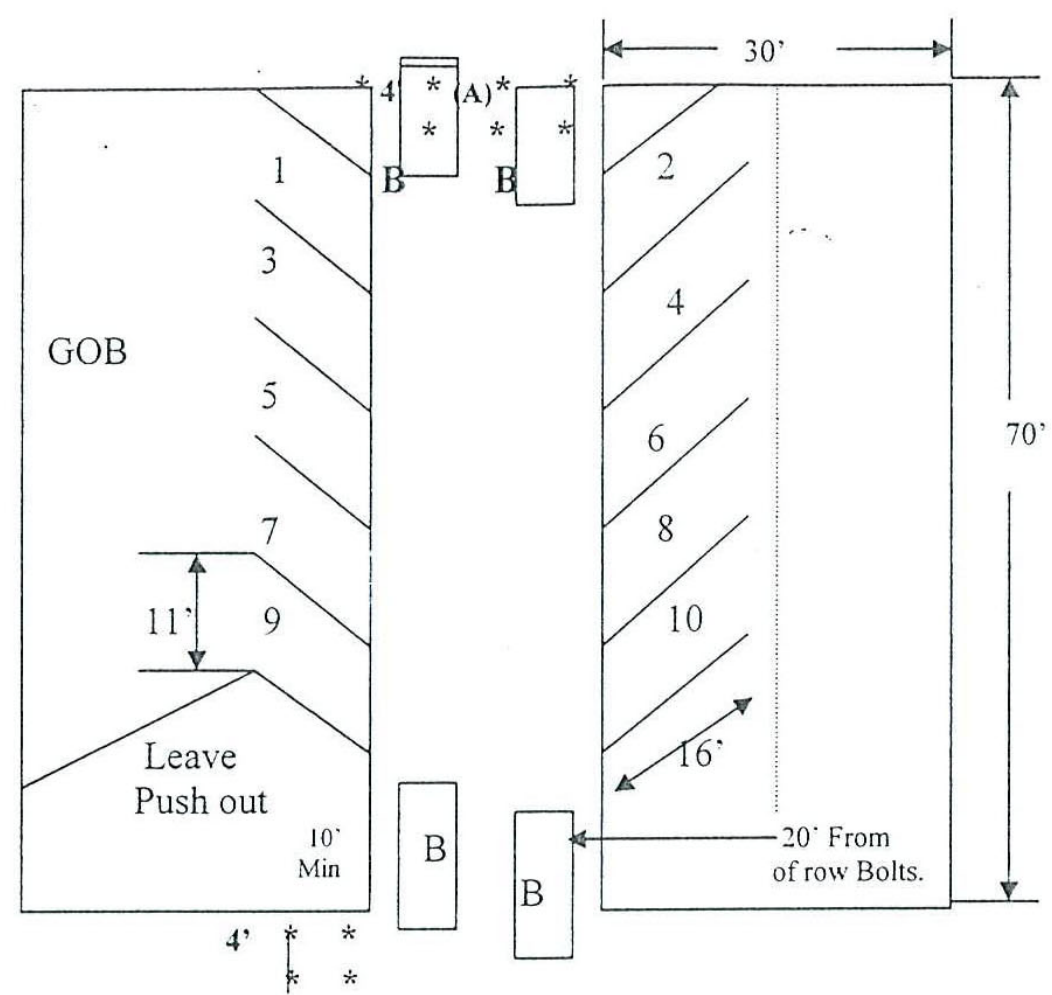

(A)

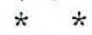

$\div \quad \%$

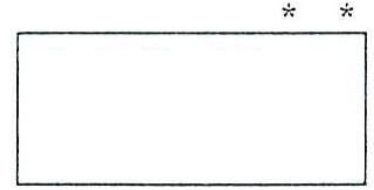

$$
\begin{aligned}
& *=\text { Breaker Posts } \\
& @=\text { Tri Set Posts }
\end{aligned}
$$

1. A minimum of eight (8) breaker posts shall be installed at locations (A) promptly after mining is complete inby.

2. MRS's shall be installed at location BB prior to mining lift No.1. and shall advanced in like manner for each subsequent lift.

3. The continuous miner shall operate in remote mode only.

4. All posts are to be installed on 4' $\mathrm{ft}$ maximum spacing.

5. 30 ' $\mathrm{ft}$ by 70 ' $\mathrm{ft}$ minimum block dimension. Blocks of larger dimensions can be mined using this sequence.

6. No persons shall be permitted inby the miner operator while the continuous miner is mining coal.

7. The pushout will not be mined.

8. This plan will not be used on 60 -degree crosscuts.

9. When tree top mining, no mining will be done in any entry if more than half of the block has been removed from the adjacent entry.

Figure 3.2. MSHA Approved Christmas-Tree Cut Sequence Utilized at Mine B 


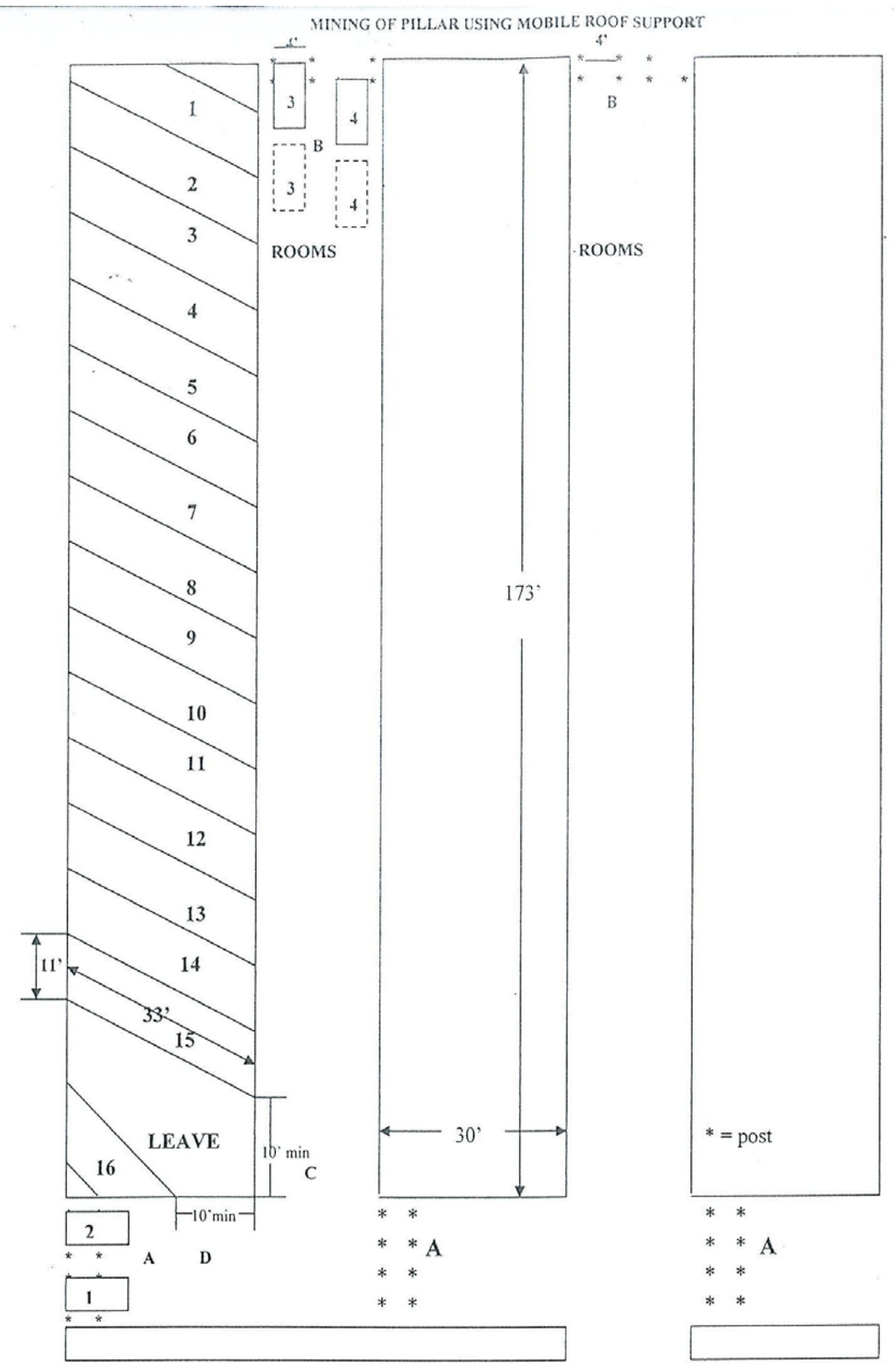

Figure 3.3. MSHA Approved Outside-Lift Cut Sequence Utilized at Mine B 
General Observations for Visit \#1. The working area around the face was well maintained and free of clutter and debris. The floor was in good condition despite a significant flow of groundwater into the mine. The cut sequence observed incorporated the Christmas tree method, as shown in Figure 3.2. The two MRS units were utilized in place of conventional turn posts, while conventional breaker posts were used in lieu of two more MRS units.

MRS Observations for Visit \#1. The MRS units were positioned inby the continuous miner and were advanced as the cut sequence progressed. The units were pressurized to approximately 2000 psi when set against the roof. During repositioning of the MRS units between cuts, units were retreated no more than one half unit length beyond the adjacent unit, in accordance with MSHA guidelines. It was not noted as to whether or not manual control on the machines had been locked out, but there was no observation of manual controls being used. During the repositioning of MRS units between cuts, non-essential personal were not present in the active mining zone and, during cutting, the MRS operators stayed one crosscut outby the pillar being mined. While there were many good observations made during this visit, there were two specific areas of concern noted

- $\quad$ When setting and lowering the MRS, the operator should be positioned in a safe location, at least 20 feet away from the units (MSHA, 2005). During repositioning of MRS units, operators were observed standing as close as 3 to 5 feet from the units they were operating,and

- While pillaring, MRS pressure gages or colored area lights should be used to monitor roof loading (MSHA, 2005). The MRS units on the section were equipped with dial gauges to monitor roof loading, however the size of the gauges and obscured visibility caused by dust and water sprays during the cutting sequence made the gauges nearly impossible to read during the cutting sequence.

Summary. Personnel on this section operated very efficiently and did not linger in the active mining zone when their presence in the area was not necessary. As seen in other mine site observations, it was not always clear who was responsible for monitoring the load on the MRS units. MRS operators and others on the section seemed to have a false sense of security due to the use of MRS units on the section. It is likely that this false sense of security is what leads miners to venture too close to the units during repositioning and to congregate in the active mining zone for unnecessary reasons.

General Observations for Visit \#2. During the second visit, the mine was extracting pillars using the cut sequence referred to as the outside-lift method. This method provides the continuous miner operator with an intact coal pillar to his back at all times during the pillar removal process, providing additional stability to the active mining zone. This method is only suitable when the pillars being mined are no wider than the allowable depth of. In this case, the miners were extracting the finger pillars that had been left from room cuts into the barrier pillar. The pillar dimensions were $30 \mathrm{ft}$ by $173 \mathrm{ft}$. 
The MSHA approved outside lift cut sequence along with location of MRS units is shown in Figure 3.3.

MRS Observations for Visit \#2. The MRS units were positioned inby the cut to be taken and were advanced no more than one-half a unit's length beyond the adjacent unit. The units were pressurized to approximately 2000 psi. There was no observation of manual controls being used. However there were two areas of concern noted during this visit.

- $\quad$ When setting and lowering the MRS, the operator should be positioned in a safe location, at least 20 feet away from the units (MSHA, 2005). During this visit, the operator was again noted standing as close as 3 to 5 feet from the MRS units while repositioning them for the next cut. This unsafe behavior is completely unnecessary and completely removes the benefit of having remotely operated MRS units. It is important to point out that the behavior was noted at the same mine during a previous visit, but the MRS operator observed during the second visit was not the same operator as previously observed.

- $\quad$ Another behavior that was of great concern was the location of the MRS operator during the cutting sequence. While coal was being cut, the MRS operator sat inby the continuous miner, using the plow of the MRS unit as a prop to lean against. This behavior was observed during the majority of the visit.

Summary. When miners were asked how they feel about using MRS units on pillaring sections, most express the large amount of trust that they have in the units. Some miners, that have had experience pillaring without MRS units, are very enthusiastic about how much easier and safer it is to pillar using MRS units. While it is nearly undisputable that MRS units significantly aid production and safety on a pillaring section, the lack of understanding surrounding their capabilities and safe operation may be a major factor in many of the pillaring fatalities and injuries associated with MRS units, due to the possible influence of overconfidence exhibited by worker performance

\section{$\underline{\text { Mine C }}$}

Mine $C$ is located in northern West Virginia. At the time of the observations, the mine was extracting coal with a mining height of approximately 45 inches. The immediate mine roof was black shale overlain by siltstone. Entries at the mine were developed to a width of approximately $16 \mathrm{ft}$. Pillar dimension was approximately $55 \mathrm{ft}$ by $55 \mathrm{ft}$. Primary roof support consisted of 4 - $\mathrm{ft}$ fully grouted roof bolts, on 4 -ft centers (Figure 3.4). 


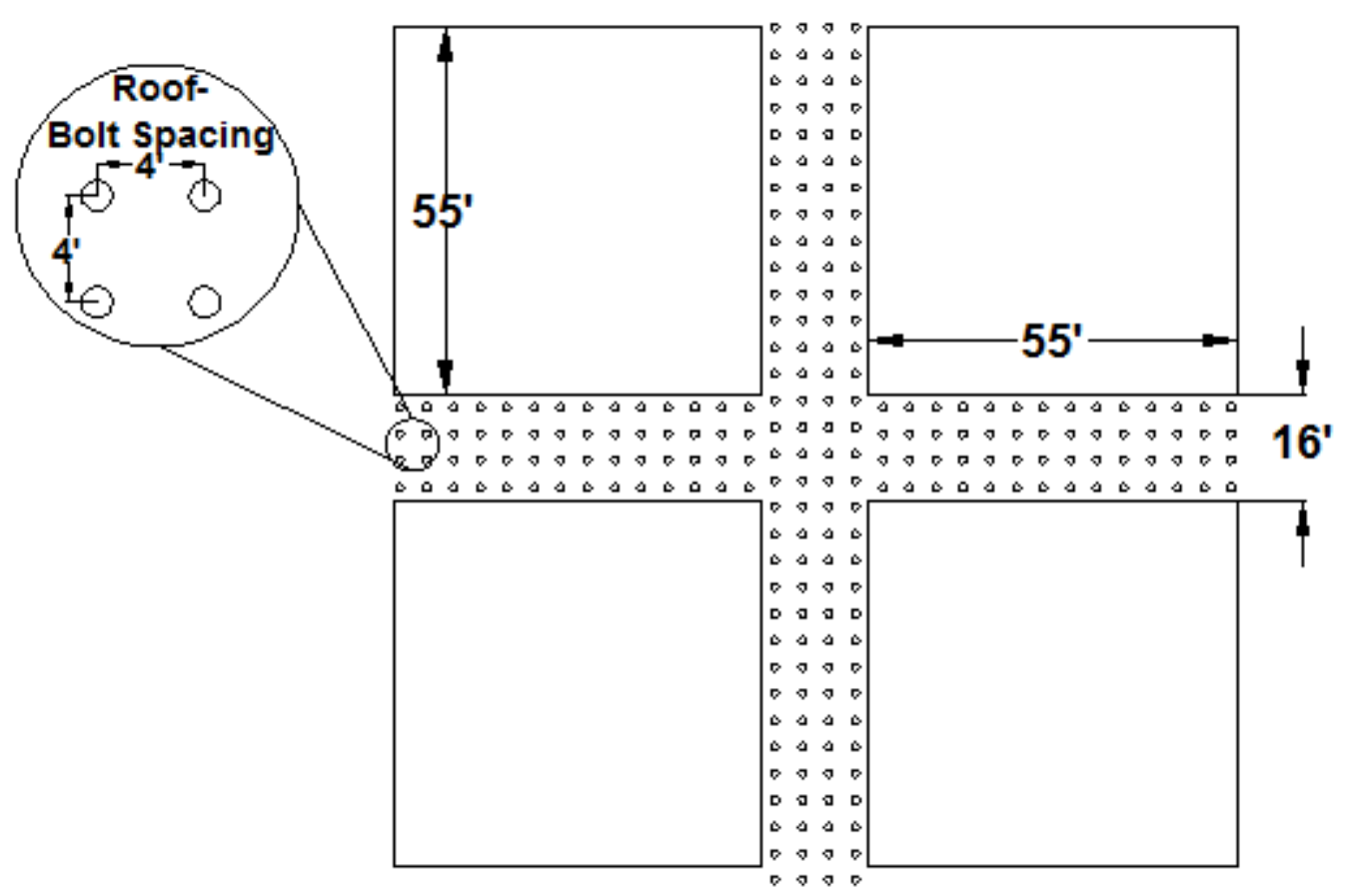

Figure 3.4. Mine C Observed Dimensions and Roof-Bolting Pattern

Supplemental support was provided by 8-ft cable bolts. Maximum overburden at this mine was approximately $500 \mathrm{ft}$. Undermining had occurred within $100 \mathrm{ft}$ of the seam being mined. This particular mine had a significant inflow of groundwater, causing the floor conditions to be very muddy and made walking somewhat difficult in places. Working conditions in this mine can be described as difficult due to the low-seam height, combined with the challenges posed by marginal roof and floor conditions. The operation employed 80 miners and produced slightly more than one million tons of coal, annually.

Mine C Observations. What distinguishes this mine from the previous site observations was the lack of MRS units on the retreating section. Instead of MRS units, the crews installed breaker and turn posts to support the mine roof and prevent falls in the gob from propagating into the active mining zone. During the positioning of turn posts or breaker posts, nearly every member of the crew was involved in the task, and the work was performed as quickly as possible. The sense of urgency to set the timbers was very obvious, and the miners tried not to spend any unnecessary time in the active mining zone than was required. While the continuous miner was cutting, timbers were being cut and supplies were being delivered for the next post setting cycle. The crew operated very smoothly.

The bowing and popping of the timbers provided the crew with both visual and audible signals that a fall was imminent, and crew members were very diligent in monitoring these signals. Due to the number of miners required in the active mining zone to set posts (as many as 5 were observed), a roof fall in the active mining zone 
poses a much higher risk of inflicting multiple severe injuries, a situation about which the miners were aware. The attitude of the miners on this section was very careful and cautious. The perception was that they were constantly exposed to hazards, resulting in high levels of attention. When visiting other mine sites that utilize MRS units, this perceived level of risk and urgency was not noticeable. Although miners seemed more alert and cautious of their surroundings, they tended to congregate as close as possible to the active intersection in between tasks, in order to observe cutting and loading as well as to monitor the bowing of timbers in the gob. While monitoring these things is important, it should be done in a manner that does not place unnecessary personnel at risk.

Summary. The marginal condition of the roof and floor, combined with the low working height, made working conditions in this mine difficult. The lack of MRS units resulted in higher amounts of exposure of workers to high-risk zones adjacent to the gob and in the active intersection. However, the miners seemed very aware of the increased risk and appeared to be very attentive to it. It was this heightened sense of awareness, presumably due to the increased perceived risk, that was absent at other pillaring sections that utilized MRS units.

\section{Job Description Observations}

On a pillaring section, it is difficult to assign a job title to every person on the section; while a miner may have a specific assignment, it does not mean that the miner might not be called upon to participate in other tasks. With that in mind, there are typically seven distinct job assignments on a pillaring section: 1) Continuous Miner Operator, 2) Continuous Miner Helper, 3) Mobile Roof Support Operator, 4) Shuttle Car Operator, 5) Foreman, 6) Scoop Operator, and 7) Electrician. It can be argued that the assignment of a timberman should be included in the previous list but, based on the mine site observations conducted for this thesis; it became evident that the task of setting timbers is assigned to anyone that is capable of assisting at the moment. As a result, since this is a high-risk activity, it is usually not an activity that any one person is designated to perform.

As shown in Figure 2.20 of Chapter 2, the continuous-miner operator was the highest risk job classification on the pillaring section, while the next highest risk was the MRS operator. While there are statistically more fatalities related to the continuous miner operator, it is important to consider the time of exposure to the hazards of working in the active mining zone. The continuous miner operator spends almost all of his time in the active mining zone, which is the reason for the higher fatality rate. The reason for the higher fatality rate among MRS operators is not as readily apparent. MRS operators should only be in the active mining zone during the necessary movement of the mobile roof supports following a cut by the continuous miner. Based on mine site observations, it can be safely concluded that an MRS operator should spend less than half of the time exposed to the active mining zone, compared to that of the continuous miner operator. When exposure times are compared, it can be argued that the repositioning of mobile roof supports may be the most high-risk activity on the pillaring section (assuming that the section operates mobile roof supports as opposed to simply a timbering roof-control 
plan). It is indisputable that the use of mobile roof supports is beneficial to strata control issues related to pillaring, and reduces the need for the high-risk task of timber setting, but fatalities are still occurring. This supports the assumptions that training, for MRS operators as well as other personnel, may be insufficient or that guidelines that are already in place are not being followed. In order to better evaluate mineworker performance, it is important to have an understanding of the workers' responsibilities. This information can benefit training in two ways. First, by simply understanding what each mineworker is asked to do can help guide training. Second, understanding each mineworker's job description can help managers tailor their observation strategies to optimize results and further benefit training.

In mining, it is common for certain tasks to be performed by whoever is available, regardless of job title. In this thesis, the following sections will describe the tasks most commonly associated with the daily routines of these particular job titles. These job summaries have been assembled based on general knowledge combined with the observations at the mines visited during the course of this research.

\section{Continuous Miner Operator}

When the continuous miner operator arrives on the section, his first task includes gathering information from the foreman or operator from the previous shift in order to address any operational or safety issues that may have been encountered on the previous shift. The operator will then inspect the workplace, check roof and floor conditions, ensure that the face area is properly ventilated, and locate and inspect all cables in the area of the continuous miner. The operator's attention is then directed toward the maintenance of the miner, ensuring that all worn bits are replaced, all lights and emergency shut offs are functioning, all water sprays are unimpeded, and all hydraulic features are properly functioning. After the miner has been inspected and approved for operation, the operator will energize the machine and check the function of the remote controls. After completing all of this, the operator will communicate his intentions to the rest of the crew in the active mining zone and begin the cut. During the course of cutting, the operator is charged with paying close attention to the roof as well as being required by law to check methane levels every twenty minutes. In between cuts, the miner operator may be assigned with miscellaneous chores, such as clearing debris from the miner or repositioning trailing cables. After the pillar is mined, the operator will then tram the miner to the next pillaring location and repeat the process.

\section{Continuous Miner Helper}

The continuous miner helper's primary function is to assist the miner operator in activities associated with the continuous miner. This may include, but is not limited to, moving and hanging electrical cable and ventilation curtains or tubing, changing bits, and servicing the continuous miner. The miner helper also plays a vital role in monitoring the roof and rib conditions surrounding the active mining zone. The continuous miner operator helper positions himself in close proximity to the operator for the majority of the cutting cycle and, therefore, the amount of exposure time to hazardous conditions is nearly identical to that of the continuous-miner operator. 


\section{Section Foreman}

The section foreman typically serves in a supervisory role, although it is not uncommon in non-union mines for the section foreman to step in and operate machinery or perform manual labor when necessary. His job is to ensure that the section operates as safely and efficiently as possible. The section foreman will hold safety meetings, train miners on proper equipment operation, observe roof and floor conditions, and make sure that mining is in compliance with the MSHA-approved roofcontrol plan as well as all other applicable state and federal regulations. During pillar extraction, the section foreman's time of exposure in the active mining zone is usually as an observer. His presence on the section is important to ensure that things are done safely and efficiently, but he does not necessarily have to be in as close proximity to the active mining zone as other members of the section crew; therefore, his exposure to hazards in the active mining zone and the resulting fatality rates for foreman on pillaring sections may be relatively low.

\section{Mobile Roof Support Operator}

The MRS operator assures that the MRS units are in good operating conditioning at the beginning of each shift. During the shift the MRS operator is responsible for repositioning the units between cuts and the relocation of the units to the next pillar, after a pillar has been completely extracted. During the repositioning of the units between cuts, the operator is exposed to the active mining zone for the duration of the task. After repositioning is complete, the operator's presence in the active mining zone is no longer necessary. Downtime between tasks should be spent at a safe location outside of the active mining zone. During the move to a new pillar, the MRS operator is initially exposed to the increased hazards of the active mining zone but, as the move progresses away from the previous active mining zone to the next pillar, the major hazard becomes the pinch points created between the rib and the MRS unit, itself. It is not uncommon for the operator to be standing in close proximity to the MRS unit while moving it to the next pillar. During the course of this thesis, a review of pillaring-related fatalities on pillaring sections was conducted. MRS operators had the second highest fatality rate of all of the job descriptions. This fatality rate is alarming when one considers that the amount of time spent in the active mining zone should be relatively low when compared to other job descriptions. The time spent in the active mining zone by the MRS operator is intermittent and, therefore, leads to the conclusion that the brief task of repositioning MRS units is a very high-risk task.

\section{Scoop Operator}

The scoop operator's activities in the active mining zone of a retreating section are intermittent, and activities outside of the active mining zone are not a concern of this thesis and, therefore, will not be covered. The scoop operator's function on a retreating 
section is primarily housekeeping and supply delivery. At the beginning or end of a shift, or at any time necessary, the scoop operator may be called upon to clean up the floor or deliver bits or other types of necessary tools or supplies. During the mine-site observations conducted during the course of this thesis, it was noted that the only time the scoop operator was present in the active mining zone with any regularity was during equipment moves that required the setting of breaker posts. During this activity, the scoop operator would deliver posts, and then assist in measuring, cutting and setting the posts. After this was completed, the scoop operator would leave the active mining zone to perform maintenance-related tasks elsewhere in the section. So while the scoop operator has a low amount of exposure to the active mining zone, his time spent there is during what may be the most high-risk time period.

\section{Shuttle-Car Operator}

The shuttle-car operator's primary duty is to transport coal from the active mining zone. Like all other equipment operators, the shuttle-car operator is responsible for inspecting the machine prior to and during his shifts, but this does not typically take place in the active mining zone of a pillaring section. Therefore, this activity is not of significance to this thesis. The shuttle-car operator, ideally, is only present in the active mining zone of a pillaring section on an intermittent basis, while accepting a load of coal. The time spent in the active mining zone per shift is related to the travel time associated with the haul route as well as the time necessary to load the shuttle car. Although shuttle-car operators have been killed in roof falls on pillaring sections, the actual shuttle car, itself, can be considered a significant hazard to other personnel, both on pillaring and advancing sections.

\section{Electrician}

During the fatality review conducted during this thesis, there were no documented cases of electricians being fatally injured on a pillaring section. Though there were no electrician fatalities, an electrician working on a pillaring section is still exposed to more hazardous conditions than would typically be encountered in a typical advancing section.

The electrician's responsibilities on the section include the positioning and maintenance of the power supply network, as well as maintenance and repair of equipment, splicing severed power cable etc. Typically, most planned maintenance is performed outby the active mining zone. This makes the electrician's presence in the active mining zone intermittent and lowers the hazard exposure rate which in turn lowers the risk associated with the job title. It is during the unplanned repairs that cannot be moved outby the active mining zone that an electrician on a pillaring section will have the highest exposure to hazards. On a section, utilizing just a continuous miner at the face and timber roof supports, these types of spot repairs would typically be limited to the repair of a damaged cable that prevented the miner from being moved outby or the repair of possibly more severe electrical problems that could render the unit immobile and necessitate spot repairs. However, as pillar extraction becomes more mechanized by the implementation of equipment such as MRS's, the amount of time an electrician 
spends performing unplanned repairs in the active mining zone has increased. For example, by implementing four MRS units on a pillaring section, the amount of equipment that continually remains in the active mining zone has been multiplied by a factor of five, and based upon this, it can be assumed that the rate of breakdowns and necessary spot repairs is sure to increase, as will the amount of hazard exposure time for the electrician.

\section{Summary}

The purpose of the mine-site observations, presented in this chapter, was to gain a better, first-hand understanding of the tasks and working conditions encountered on typical pillaring sections in Central Appalachia, as well as, to determine if unsafe performance trends, identified during the fatality reviews, were present at these operations.

Through the course of these observations, it became evident that unsafe performances that have previously contributed to fatalities are commonplace, even at mines considered to be safe and productive. So while many miners at these mines feel confident that they are working safely, it can be inferred that the only difference in these mines that are operating effectively and the ones that have experienced severe accidents is the likely reduction of geotechnical risk factors that have made accidents less frequent because, from the standpoint of performance-based risk, these mines are still operating at significant levels of risk. Miners are still taking chances beyond the recommendations and regulations meant to promote safety. Performance was, in many cases, very similar to what was observed in past fatal accidents, indicating that, in many operations, possibility still exists for a failure of engineering controls to result in a serious accident due to the failure to control residual risk. Some of the unsafe performances which necessitate attention include:

- unnecessary congregation in or near the active mining zone,

- inattention to MRS dial gauges to monitor roof loading conditions,

- unsafe positioning during downtime,

- failure to operate MRS units and maneuver trailing cables in accordance with MSHA standards,

- horseplay, and

- failure to perform in accordance with MSHA approved roof control plan.

As previously stated, these performance issues have all contributed to injury and accidents in the past. Their presence indicates either inadequate training or a lack of compliance, both of which can be addressed through structured performance observation. Therefore, in order to effectively identify and address these performancebased risks, the structured performance observation technique that was developed will be introduced in Chapter 4. 


\section{CHAPTER 4. THE DEVELOPMENT OF A WORKPLACE SAMPLING STRATEGY FOR PILLARING SAFETY}

\section{Introduction}

The purpose of this chapter is to develop a sampling strategy for safety-training specialists to gather observable performance information of the mineworkers on a pillaring section in a systematic manner. The results of this sampling strategy can be used to assess mineworker exposure to pillaring hazards, as well as to identify the potential consequences resulting from these workplace risks. This strategy will help mine management identify conditions or behaviors that may lead to accidents or injuries, thereby making training more effective by targeting problem areas.

\section{Description of the Strategy}

Comprehensive hazard identification is crucial for the success of any risk management program. Without it, the existence of risks would be difficult to accurately assign and control. As discussed in previous chapters, there are many types of risk, and various types of risk factors. This chapter will describe pillaring-related risk as a form of engineering risk or performance-based risk. Engineering risks are described as the physical factors related to mining that would typically be addressed by technical personnel. Performance-based risks are those undesired situations that would be addressed by managers who direct or educate the workforce, such as a foreman, safety manager, or trainer. While these two types of risk are separate, the overall goal is to control both types of risk to the point where, if there is a performance - or engineeringbased failure, one could compensate for the other. For example, engineers design roof control plans to prevent roof failures but, due to the variable nature of mine roofs, it is not uncommon to have failure. This is why it is important to emphasize safe mineworker performance in order to reduce the residual risk caused by the inherent variability of roof and strata conditions.

\section{Engineering Risk Factors}

Engineering risk factors are physical factors that should be addressed prior to mining and continuously monitored throughout the course of mining, such as depth of cover, methane liberation rates or roof conditions. By identifying these pre-existing conditions, the severity or risk of negative interactions that may be caused can be determined by the technical staff. By analyzing the inherent risks, appropriate engineering controls can be put in place to minimize the overall risk.

\section{Performance-Based Risk Factors}

While some workplace hazards related to retreat mining are due to materials handling, machinery movement, etc., most are related to the physical conditions that 
are primarily addressed by engineering controls but, as stated earlier, retreat mining has numerous hazards and, although best efforts may be directed toward the controls of risk through engineering, residual risks still exist. This residual risk is beyond the scope of engineering control, but can be either minimized by the effectiveness of personnel managers and, ultimately, the performance of the miners, themselves. That is the reason why this form of risk is referred to as performance-based risk in this chapter. As a result of the extensive review of pillaring fatalities in Chapter 2, it was observed that most sources of pillaring fatalities were related to a failure that was intended to be controlled through engineering, such as a roof fall but, if equipment had been operated in accordance with MSHA regulations or if the miners had been positioned in safer locations, the consequence of the engineering-control failure could have been significantly reduced. Therefore, while engineering control is the initial method of minimizing risk on a pillaring section, the control of any residual risks by the miners, themselves, is just as important in preventing fatalities and serious injuries.

\section{Development of the Sampling Forms}

As previously noted, in order to successfully manage risk, hazards must be identified. In the area of pillaring, these hazards include physical factors as well as workplace safety and performance factors. To advance the concept of risk management, four observation sampling forms and one pre-mining risk matrix form have been developed in order to enable safety managers to gain a qualitative and quantitative understanding of the performance of their employees on pillaring sections. The risk matrix and observation forms are listed below in the order that they will be addressed in this thesis:

- Pre-mining risk matrix,

- Unsafe worker performance observation,

- CM-operator observation,

- MRS-operator observation and,

- Mineworker-location observation.

The forms presented and discussed in this chapter are condensed; full-sized forms to be copied and used in the field appear in Appendix B. The information gathered with these sampling forms will enable engineering and safety personnel to identify unsafe performance, whether it is an isolated incident or a pattern of unsafe behaviors. Based on the identification of these incidents or trends, managers will be more effective in addressing these issues through engineering controls or training.

The four observation sampling forms were developed based upon the study of fatality statistics and reports, as well as on-site worker observation. There are two forms that are more encompassing and document the performance or location of every person entering a pillaring section. The remaining two observation forms are more specific. They have been developed to track the positioning of the miners that perform job descriptions that have proven to be particularly high risk, based upon the tasks previously cited throughout the course of this thesis. These specific job-description observation forms were developed to provide a quantitative analysis, so that information 
can easily be graphed or charted for presentation or comparison. Quantitative results also remove some of the potential inaccuracy that is inherent with purely qualitative assessment.

A pre-mining risk matrix was also developed to enable engineers and safety managers to qualitatively assess risk(Figure 4.1). The physical-risk matrix was developed with engineering-related risk factors in mind, and provides a template for engineers and management to develop their own physical-risk matrices at their individual operations. Copies the forms developed for the sampling strategy are provided in Appendix B.

\section{Pre-mining Risk Matrix}

Figure 4.1 represents the pre-mining risk matrix form developed for the initial identification and assessment of pre-mining risk factors. 


\begin{tabular}{|c|c|c|c|c|c|c|c|c|}
\hline & & Present & Absent & $\begin{array}{l}\text { : } \\
\text { : }\end{array}$ & 窎 & 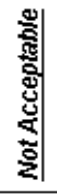 & $\begin{array}{l}\frac{\text { Probability of }}{\text { Neqative }} \\
\frac{\text { Consecquences }}{(N A, V L, S L, P, U)}\end{array}$ & Motes \\
\hline 1 & Roof Conditions & & & & & & & \\
\hline \begin{tabular}{l|l}
$\underline{2}$ \\
\end{tabular} & Floor Conditions & & & & & & & \\
\hline 3 & Rib Conditions & & & & & & & \\
\hline 4 & Overmining & & & & & & & \\
\hline$\underline{\underline{5}}$ & Undermining & & & & & & & \\
\hline$\underline{6}$ & \begin{tabular}{|l} 
Abandoned \\
Adjacent $M$ me
\end{tabular} & & & & & & & \\
\hline \begin{tabular}{l|}
$\underline{7}$ \\
\end{tabular} & \begin{tabular}{|l|} 
Consistencv of \\
Pillar Dimensions
\end{tabular} & & & & & & & \\
\hline$\underline{\underline{8}}$ & Iimbering Plan & & & & & & & \\
\hline$\underline{\underline{g}}$ & \begin{tabular}{|l|} 
Mobile Roof \\
Support Plan \\
\end{tabular} & & & & & & & \\
\hline 10 & Depth of Cover & & & & & & & \\
\hline$\underline{11}$ & \begin{tabular}{|l} 
Distance from \\
Outcrop
\end{tabular} & & & & & & & \\
\hline 12 & $\begin{array}{l}\text { ARMPS Pillar } \\
\text { Stability Factor }\end{array}$ & & & & & & & \\
\hline$\underline{13}$ & Ventilation & & & & & & & \\
\hline$\underline{14}$ & \begin{tabular}{|l|} 
Methane \\
Liberation \\
\end{tabular} & & & & & & & \\
\hline 15 & $\begin{array}{l}\text { Expected } \\
\text { Cavabisity }\end{array}$ & & & & & & & \\
\hline$\underline{16}$ & & & & & & & & \\
\hline$\underline{17}$ & & & & & & & & \\
\hline$\underline{18}$ & & & & & & & & \\
\hline
\end{tabular}

Figure 4.1. Pre-Mining Risk Matrix

Successful risk management requires that efforts should be directed toward controlling the risk factors that need to be immediately addressed and, after the most urgent risk factors are controlled, refocusing on factors of lesser risk. The pre-mining risk matrix form enables engineers and mine planners to identify hazards and qualitatively assess the risk associated with their individual operations, so that these risk factors may be controlled in the most effective manner possible. While care was taken to include as many pre-mining physical risk factors as possible, this risk matrix is merely a template that can be used by itself or modified by the addition of other hazards in order to address issues at individual operations.

The risk matrix consists of risk sources and risk factors. The presence of a risk source, alone, represents some level of risk, while risk factors are physical properties that contribute to the level of risk associated with a risk source. In underground mining, there are risks, such as roof falls or rib rolls, that are present at 
every operation and, due to the complex nature of such a risk, it is more feasible to note the magnitude, presence or absence of risk factors that affect these hazards. This methodology can be seen in the risk matrix presented in Figure 4.1. As shown, there are five ways to categorize risk sources or risk factors in this risk matrix: acceptable, marginal, not acceptable, present, or absent. There is also a column noting the likelihood of negative consequences related to the hazard or risk source, based on the qualitative assessment of risk managers. This is why it is crucial for members of a riskmanagement team to be experienced in their designated areas, to enable them to accurately assess risk based upon conditions rather than merely on statistical data.

As previously noted, the pre-mining risk matrix developed in this thesis provides engineers and management with a blueprint for qualitatively assessing risk. The matrix presented was developed with pre-mining considerations, but this type of risk assessment is very flexible and can be modified to assess many different types of risks. The key to accurately assessing risk using this qualitative style of risk matrix is to ensure that every hazard or risk source on the sheet can be adequately judged by a member of the assessment team.

The risk matrix is designed to yield information regarding the presence and likelihood of consequences related to risk sources and risk factors. Since there are risk factors that are always present, such as roof conditions, and risk factors that are not always present, such as overmining, there are two different ways to identify the presence of a risk. In a risk that is always present, only the magnitude of the risk source and the likelihood of negative consequences need to be addressed. For other sources of risk, such as the use of MRS units or timbers as the main method of roof support on the section, the necessity to rate the acceptability is not necessary. It should only be indicated as present or absent and then the consequences of using one method over the other should be reviewed and indicated in the last column, since the methods have unique benefits and risks associated with each.

In conventional risk matrices, it is common to see a numerical ranking of hazards based on the severity of the consequence and probability. In this risk matrix, assigning a risk number based on consequence is less effective, since these risk sources are based on only a few complex hazards that must be assessed according to the many factors affecting them (risk factors). These hazards all have proven the ability to cause severe injury and death, so the severity of the consequences is clearly established and remains constant. Therefore, it is more effective to identify the risk factors that are most likely to impact the hazard. That is the purpose of estimating the likelihood of negative consequences. The term likelihood is used instead of probability, due to the fact that, in most cases, it is very difficult to obtain accurate statistical data on these risk factors. The assessment must be based entirely on the expertise of the members of the assessment team.

By performing this type of assessment prior to pillar extraction, the mining plan can be designed to account for the anticipated issues and, in some cases, the decision to pillar may not be chosen. By doing this prior to mining, the risk of encountering these risk factors unexpectedly or in an unprepared manner is reduced.

\section{Performance Observation}


In order for safety managers and training personnel to effectively manage performance-based risk, it is crucial that they observe and document mineworker performance, in order to accurately address undesirable performances during training sessions. In risk management terminology, these observations would serve as the hazard-identification and risk-assessment process, and the training that results from these observations can be considered the risk controls. A safety manager that can accurately identify performance-based risk should be much better equipped to confront and control these risks. During the course of this thesis, four separate observation strategies have been developed. One is a qualitative assessment of the overall performance of the crew on the section, highlighting general unsafe behavior or unsafe conditions. The remaining three were developed as a more quantitative assessment of risk based on hazard exposure.

Unsafe Worker Performance Identification. Figure 4.2 represents the unsafe worker performance identification form, to be utilized as the initial step of the mine worker performance observation strategy. 


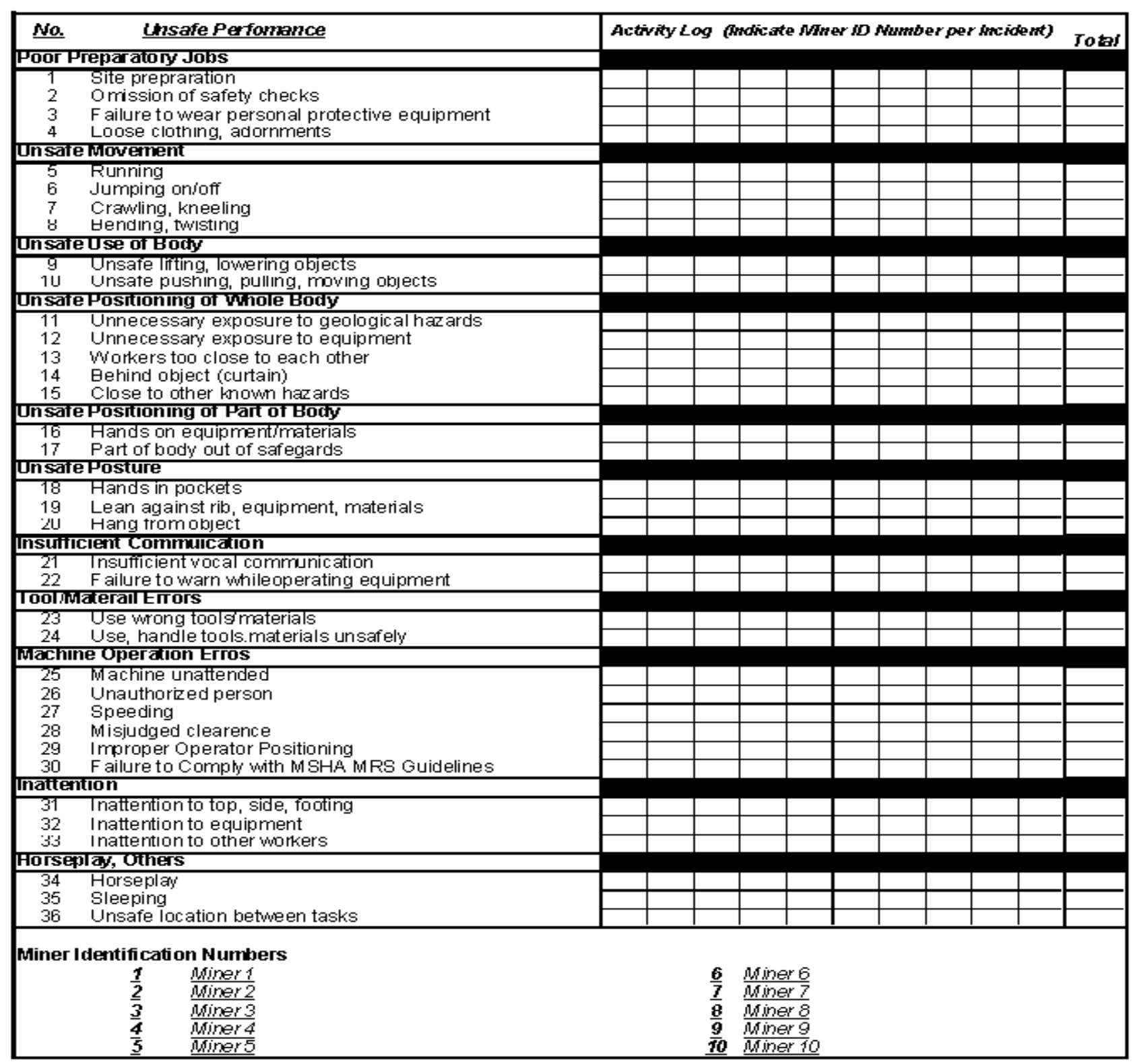

Figure 4.2. Mine Worker Activity Form

This form is a worker performance observation form adapted from Bise, et. al. (1993); it has been slightly modified to cover pillaring-specific tasks. The design of the sampling form facilitates its use, and can provide a very effective record for assessing a section. The form provides information that can be used to focus further observation, making it very useful where time and resources are limited. In this form each miner is assigned a number. If unsafe performance is observed, the number of the miner observed should be logged in the designated cell for each incident of unsafe performance. After the form is completed, it will be possible to identify the frequency of specific observed unsafe behaviors, as well as identify miners that perform in unsafe manners. This will be especially helpful for targeting certain tasks or certain persons for training purposes. 
Continuous-Miner Observation. As mentioned in Chapter Two of this thesis, there are more fatalities of continuous-miner operators and continuous-miner helpers on pillaring sections than any other job description. This is why a specific observation form to accurately identify and record unsafe behavior and work patterns in a manner that lends itself to quantitative results, rather purely general qualitative observations, was developed (Figure 4.3).
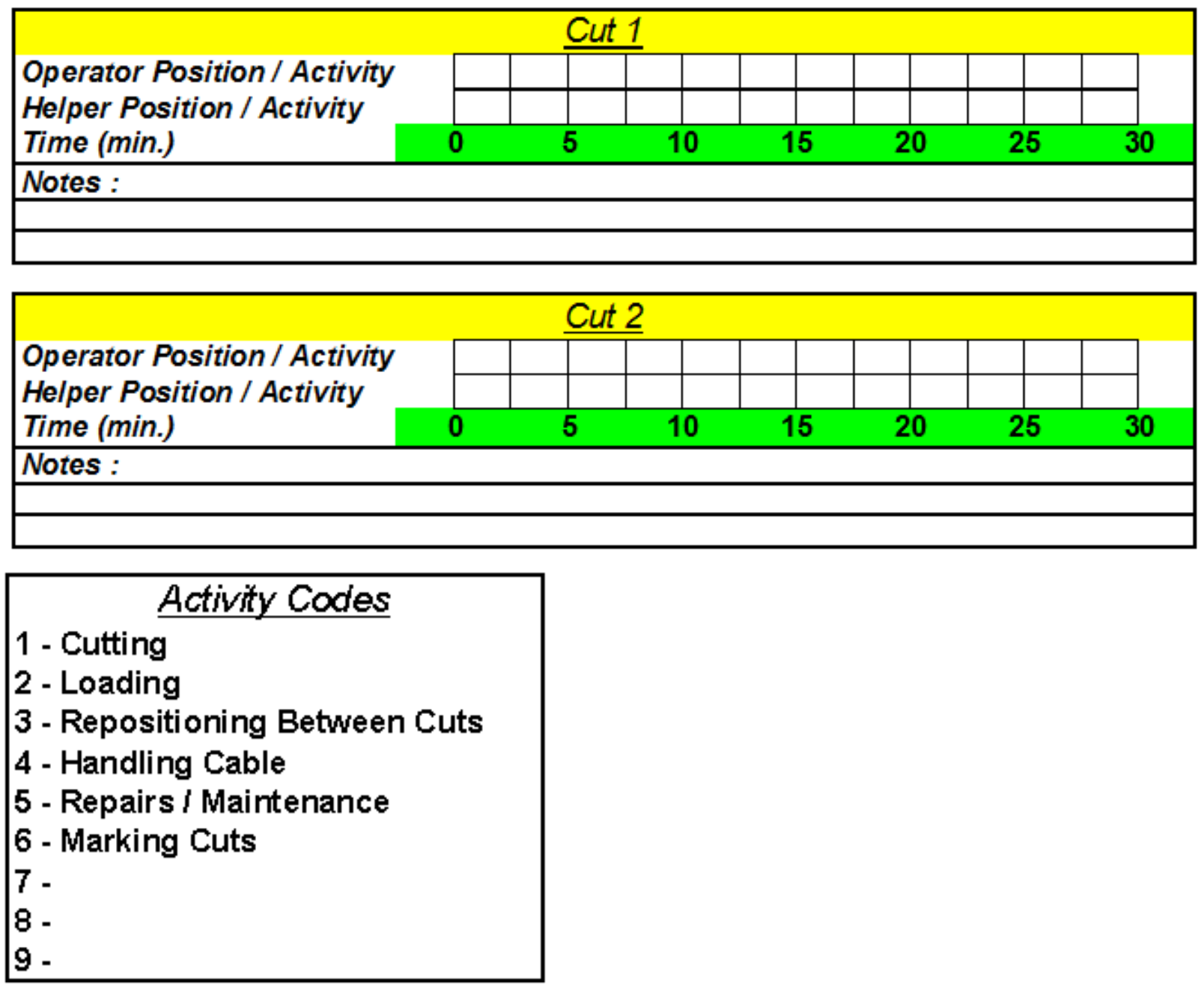

Figure 4.3. Example of the Continuous-Miner Observation Form

These quantitative results can provide management with not only with documented evidence of behavior trends or undesirable performance but, also can provide an accurate description of desirable or undesirable work patterns and times of exposure. This particular observation form divides the work area of the miner operator and miner helper into three zones across the entry and labeled alphabetically from the gob side to the active intersection (Figure 4.4). 


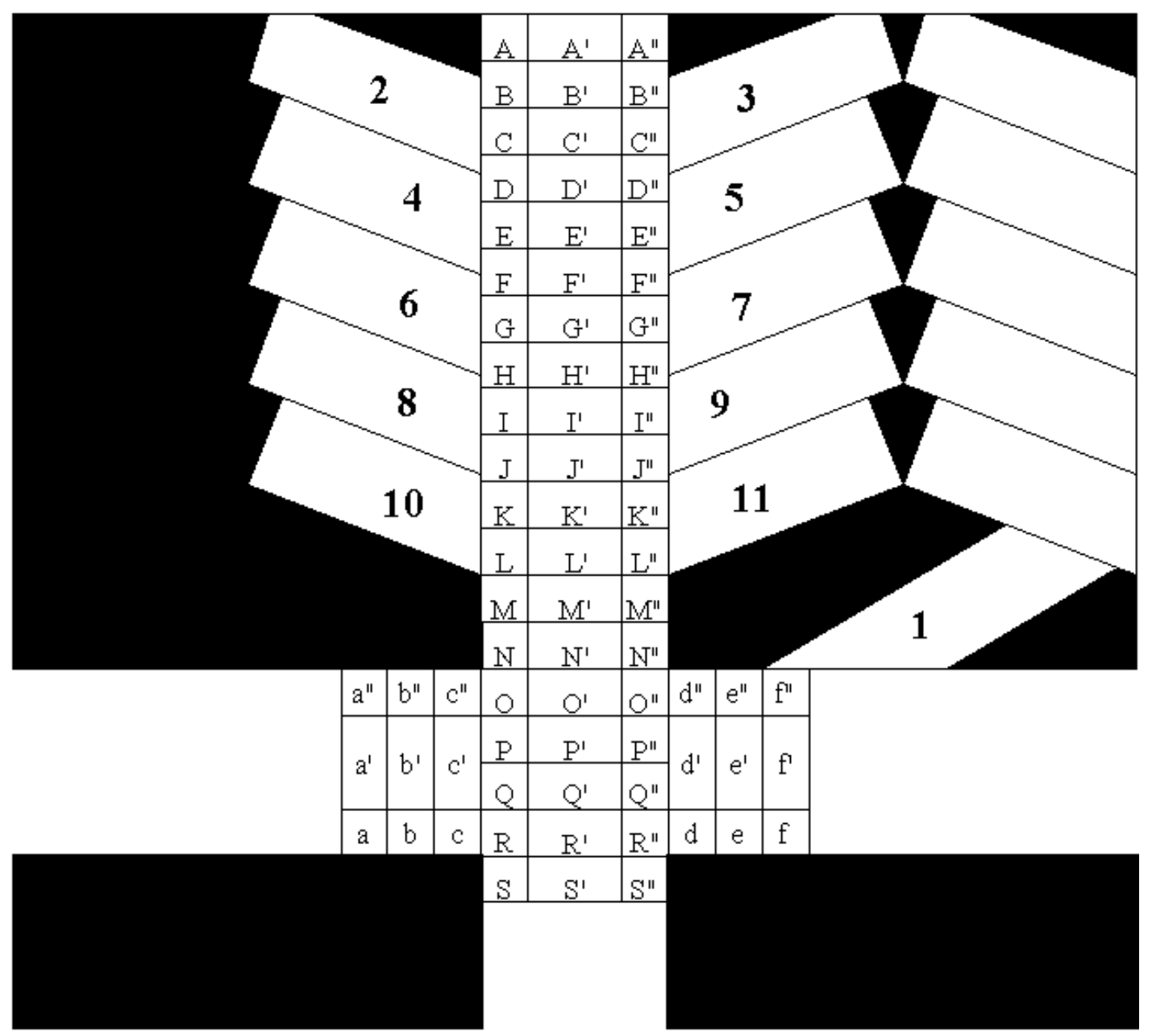

Figure 4.4. Example of the Zone Division for Continuous-Miner Observation

The three zones, labeled X, X', and X", are designed to identify the location of the operator or helper at anytime during the mining of the pillars. Zones X and X" are located directly adjacent to the ribs and extend five feet or more out into the entry, and are five feet long parallel to the entry. The remaining middle section of the entry is also five feet long parallel to the entry and is labeled as Zone X'. The capital letters indicate zones that are designated for entry positions while lowercase lettering is reserved for positioning within a crosscut. Three zones were selected instead of two, because it is important to know if the operator or helper is positioned towards the middle or the side of the entry during curtain tasks, rather than just noting whether they were on the right or left side. This is important because this worksite is very dynamic with frequent continuous miner directional changes, as well as varying loading angles to accommodate the position of the continuous miner. As a result, the operator and helper must continuously change vantage points and operational positions. This observation strategy enables the documentation of position, time, and tasks based on the logging of activity and location code onto the timeline designated for each cut. Identifying poor positioning and excessive hazard-exposure times enables safety managers to more accurately identify and address problem areas. The form (Figure 4.3) is arranged 
according to cut numbers and can easily be tailored for any cut sequence. Each cut has a timeline assigned to it. As time progresses, the observer should log the location and activity of the miner operator and the miner operator helper versus the time. At the conclusion of the observation, information can easily be extracted from the form and placed in any spreadsheet program for analysis, as shown in Chapter 5.

As shown in Figure 4.4, the capital letters run parallel with the entry while the lowercase letters are in the crosscut. Any letter with the single prime designation is in the center of the entry. Capital letters without a prime, or double prime designation are located on the left side of the entry facing inby. The capital letters designated by a double prime are located on the right side of the entry facing inby. In the crosscut, the lowercase letters with the double prime designation are adjacent to the pillar or pillars being mined. The prime designated lowercase letters are in the center of the crosscut, and lowercase letters without any prime or double prime designation are located adjacent to the first pillars outby the pillar or pillars being mined.

After a map of the pillaring section has been divided into zones, as previously described, and cuts labeled, the observer must then select a safe vantage point and begin observing and recording data on the timeline. The cut timeline will vary from mine to mine but, for this example, it has been set at thirty minutes, indicating that, as long as all activities related to the particular cut are completed in thirty minutes or less, it should be recordable on this timeline. For cut time in excess of 30 minutes, additional forms can be added. The method of recording time and activities is as follows; 1 ) as the subject moves into a zone, place a line from top to bottom of the timeline, 2) following the line place the code representing the zone and the activity being performed, 3 ) if the activity or zone changes draw another vertical line that corresponds to the time the change occurred, and repeat the process for the remainder of the cut.

MRS-Operator Observation Form. According to the research performed in Chapter 2, the mobile roof-support operator (MRS operator) has the second highest fatality rate of the job descriptions on the pillaring sections (behind the combined continuous-miner operator and the continuous-miner helper fatality rate). This fact, alone, warrants a more in-depth analysis of this job description, but the job may even be a higher risk than the fatality statistics indicate. Based on the observations conducted during mine site visits, the MRS operator spends far less time in the active mining zone than does the continuous-miner operator, but the fatality figures for these two job descriptions are very close. This indicates that while the MRS operator is in the active mining area, the tasks being performed must be very high in risk and, therefore, warrant further investigation through a specialized observation strategy. Based on information gathered during the literature review, as well as on-site visits, it is very clear that proper positioning and machine operation during MRS moves play a major role in the risks to which the MRS operator is exposed. Another factor is the operator's location and behavior during the downtime between MRS repositioning. The observation strategy developed for the MRS operator will yield quantitative information regarding the time spent in different areas of the active mining zone, as well as a measure of the operator's performance while in these areas.

The MRS-operator observation form (Figure 4.5) is similar to the CM-operator form with only slight variations. 

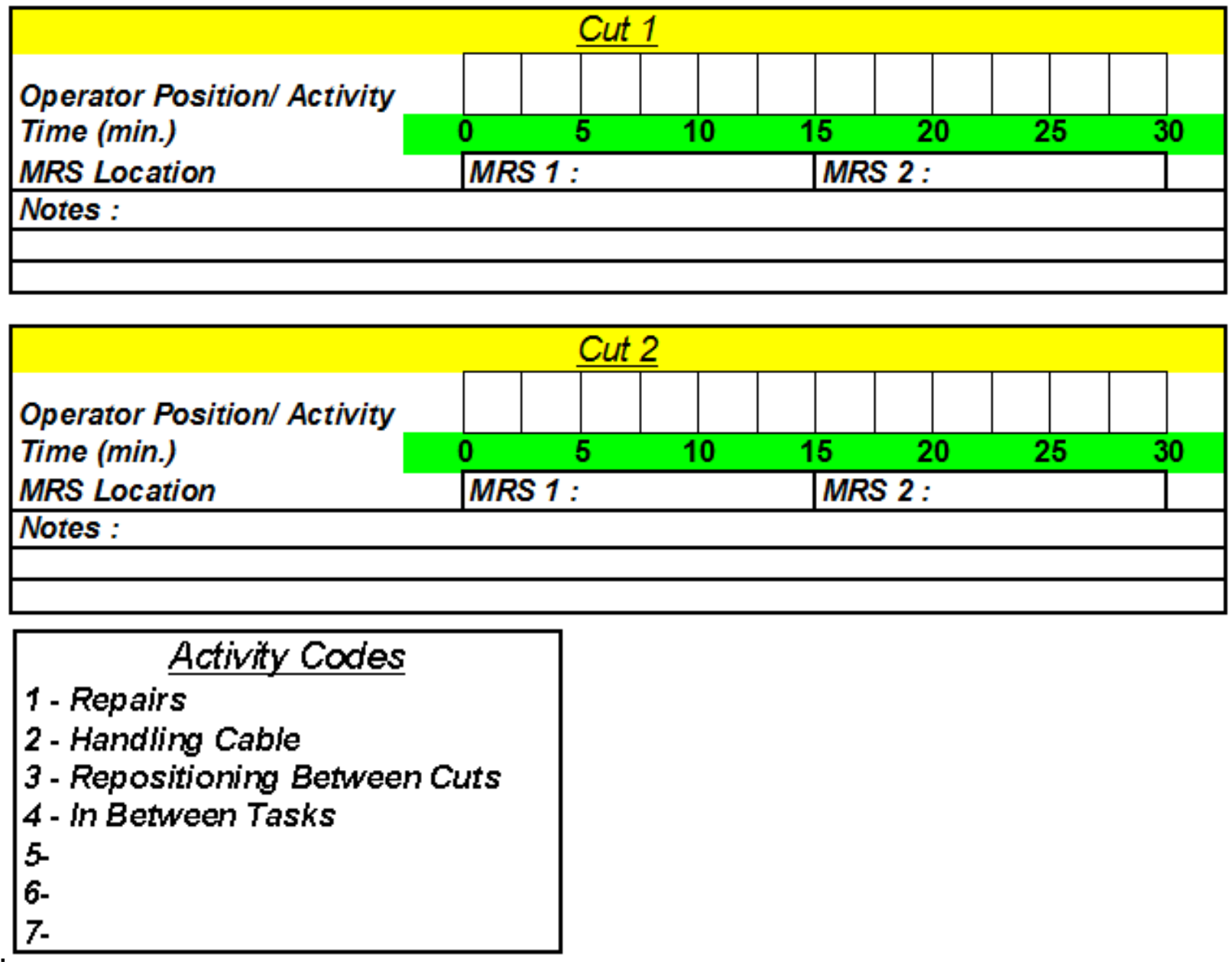

Figure 4.5. Example of the MRS Observation Form

The MRS-operator form is designed to locate the MRS operator during MRS operation, as well as when no tasks are being performed. The form also enables the documentation of the location of the inby MRS units in relation to every cut. This provides data regarding the placement of the units for every cut, the proximity of the operator to the units, and the operator's overall position in the area. Thus, unsafe locations of the operator, improper movement and placement of units, and unnecessary loitering of the MRS operator in the area following the repositioning of the units, can be identified. The MRS observation form is designed to account for the time, location, and activity of an MRS operator when moving in the any of the zones designated on the map shown in Figure 4.6. 


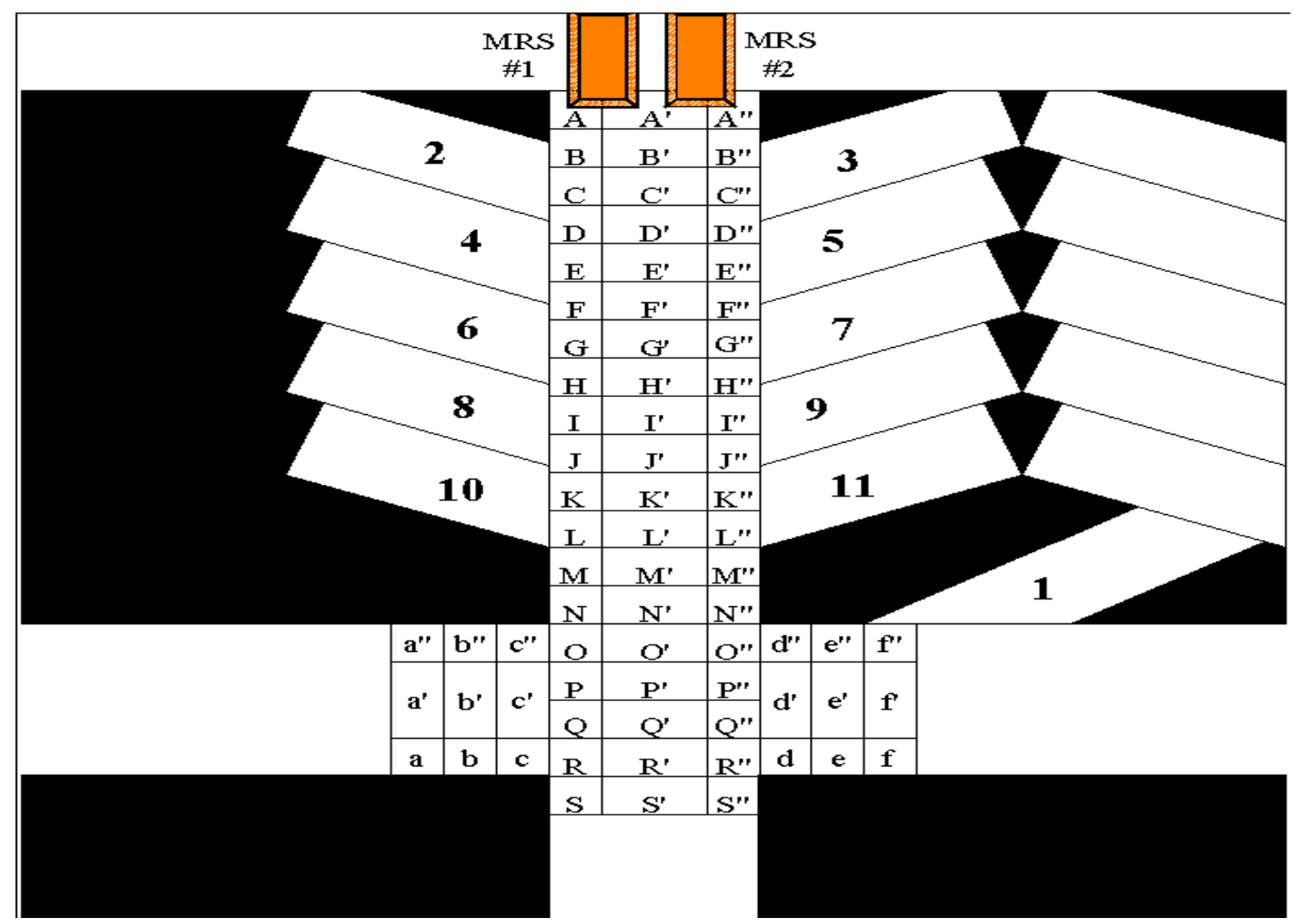

Figure 4.6. Example of the Zone Division for MRS Observation

The form also accounts for the position of the individual MRS units in relationship to a specific cut. The MRS location is important because it enables managers to identify whether the MRS units were properly positioned on a cut -by-cut basis. The MRS unit location also provides a reference point that can be used to approximate the distance between the MRS operator and the units. Figure 4.6 represents an example of how a section would be divided into segments to be logged onto the observation form. As a cut is started, the position of the MRS units should be indicated by selecting the zone that the outby facing end of the units are occupying. As the cut sequence progresses, the position of the MRS units, along with the position of the operator, should be logged. Any time spent in the immediate face area between cutting cycles should be documented in the same manner, using the code corresponding to the task that was performed. The activity should be placed in the in the area designated for the last cut completed.

Mineworker-Location Observation Form. On a pillaring section, as in all types of mining, there are areas of higher risk. These risks may be attributable to sources such as strata instability, equipment operations or many other risk sources. During the fatality study conducted for this thesis, it became clear that it is necessary to monitor the movement and activities of mineworkers throughout the pillaring section. 


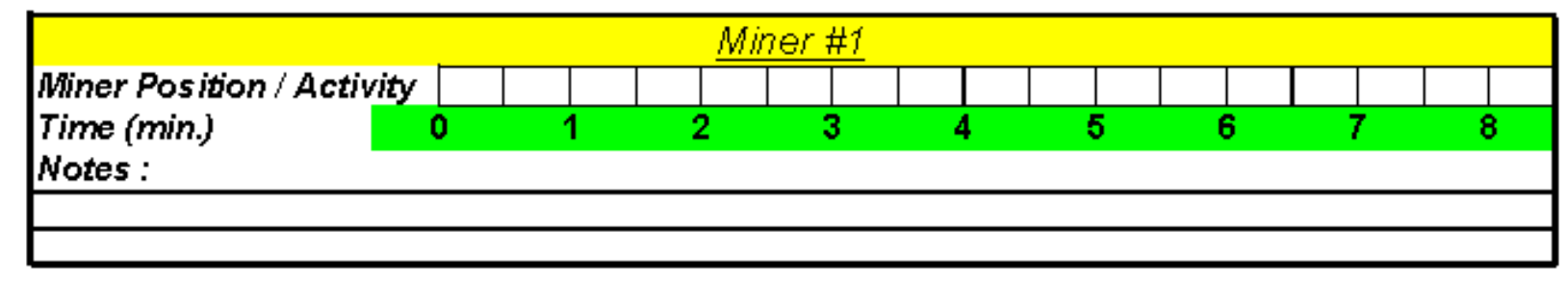

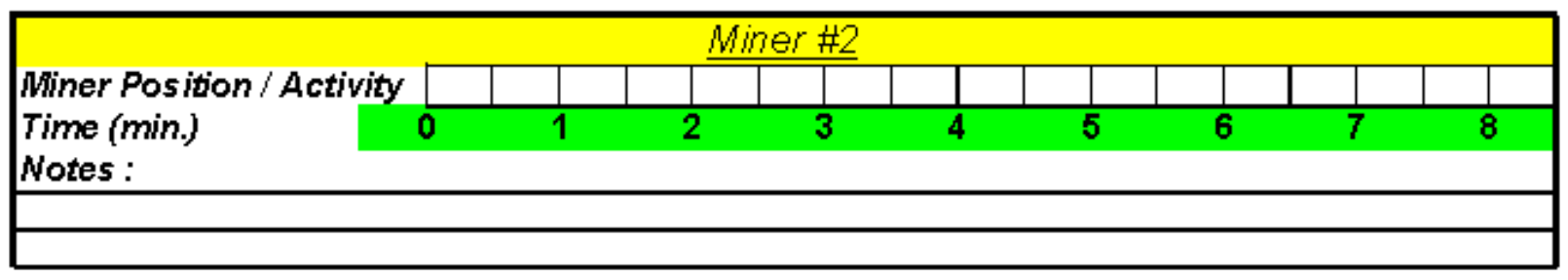

\begin{tabular}{|ll|}
\hline \multicolumn{2}{|c|}{ Activity Codes } \\
A. Operating CN & J. Between Tasks \\
B. Operating MRS & K. Operating Scoop \\
C. Observing & L. Marking Cuts \\
D. Handling Cable & M. \\
E. Hanging Curtain & N. \\
F. Checking Air Quality / Flowrate & O. \\
G. Operating Shuttle Car & P. \\
H. Setting Posts & Q. \\
I. Service / Repair & R. \\
\hline
\end{tabular}

Figure 4.7. Example of the Observation Form for Miner Observation

Improvements in mechanization, strata control and communication have greatly contributed to reducing the risk of many hazards on a pillaring section. However, these risks cannot be eliminated, and they still have the ability to cause severe consequences. One simple and highly effective method of controlling risk is to minimize exposure of personnel to sources of risk. In the case of pillar extraction, the sources of risk are concentrated in and around the active mining zone. This observation strategy is similar to the previous strategies in that it accounts for the location and behavior of personnel but, unlike the previous strategies that target small areas and specific tasks or job titles, this strategy accounts for all personnel that pass through the active mining zone and the area adjacent to the active mining zone in the direction of mining. As shown in Figure 4.8, the active mining zone and surrounding areas have been divided into eight zones and are labeled on the section map. 


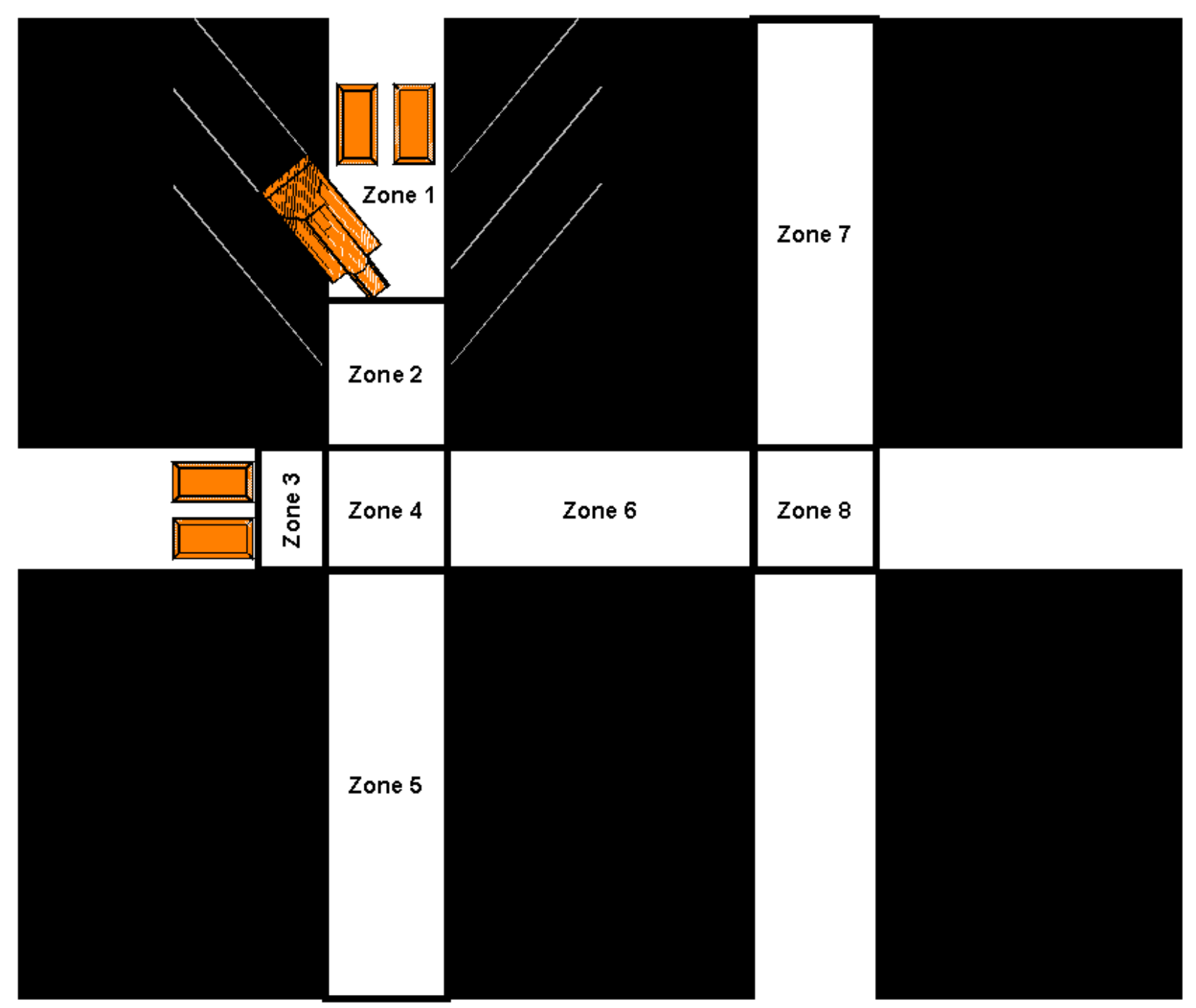

Figure 4.8. Division of Pillaring Section for Observation

These zones have been targeted as high-risk zones based on the proximity to the active intersection, proximity to active gob, likelihood of machinery traffic or other known hazards. Zone 1 is designated as the area inby the continuous miner, and Zone 2 is the area outby the continuous miner to the first outby intersection. Zones 1 and 2 will shift further outby as sequential cuts are taken; however, the remaining six zones will not change until a move is made to the next pillar in the mining sequence.

Completing this form will require at least two separate vantage points in order to cover all areas. This can be accomplished by sending two observers to collect information or by performing the observation twice from different locations by one person. The strategy for observing is different than that used for previous forms. Instead of focusing on one or two specific miners, this observation form can be used to track everyone on the section as they enter and exit the designated zones. As a miner enters a zone, the observer should move to the timeline for that specific miner, record on the timeline when the zone is entered, and what task is being performed there.

The information gathered by this observation strategy will enable safety managers to identify unsafe performances such as: 1) congregating in the active mining 
zone, 2) non-essential personnel on a section, 3) poor positioning of personnel during downtime between tasks, and 4) poor positioning of equipment operators. Engineers and safety managers must recognize that accidents do occur and the potential of a fatality is always present. This is why information regarding positioning, time of exposure, and performance is so critical to minimizing the residual risk that cannot be controlled through engineering methods. Upon completion of this observation strategy, a safety manager will have a quantitative analysis of the time of exposure of every person that moves through the designated areas. Their tasks and behavior while in the area will be clearly documented, and the information will enable safety managers to specifically target certain tasks or personnel to reduce unnecessary exposure and increase the safety and productivity of the section.

\section{Revised Sampling Forms}

In order to develop a performance sampling strategy that would provide real benefit to safety and training, it is necessary to make the strategy very practical and straightforward to use. After further review and discussion with safety professionals, the strategy presented earlier in this chapter was deemed too complex and lengthy to have practical application. Therefore, the strategy was modified to improve its applicability. The results of the modification yielded a performance sampling strategy that is less complex, yet still provides a high level of detailed information when performed correctly. The final strategy reduced the number of recording forms from five to three and reduced the complexity of dividing the active mining zone. Also, the Pre-Mining Risk Matrix and Unsafe Performance Observation Form were slightly modified to better suit the needs of safety trainers. The following list represents the final performance sampling strategy.

- Pre-Mining Risk Matrix,

- Unsafe Performance Observation,

- Activity and Location Monitoring.

\section{Pre-mining Risk Matrix}

The pre-mining risk matrix shown in Figure 4.9 is very similar to the previous premining risk matrix, but has been modified slightly to facilitate its use as well as improve its flexibility, since the previous matrix's layout did not provide sufficient capability to document pre-mining conditions. 


\begin{tabular}{|c|c|c|c|c|}
\hline & & 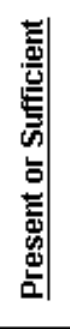 & 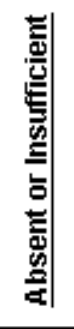 & $\begin{array}{l}\text { Notes (including Probability of Neyative Consequences) } \\
\text { (NA, VL, SL, P, U) }\end{array}$ \\
\hline 1 & Roof Conditions & & & \\
\hline 2 & Floor Conditions & & & \\
\hline \begin{tabular}{|l|l|}
$\underline{3}$ \\
\end{tabular} & Rib Conditions & & & \\
\hline 4 & Overmining & & & \\
\hline$\underline{5}$ & Undermining & & & \\
\hline$\underline{6}$ & $\begin{array}{l}\text { Abandoned } \\
\text { Adjacent Mine }\end{array}$ & & & \\
\hline & \begin{tabular}{|l|} 
Consistency of \\
Pillar Dimensions
\end{tabular} & & & \\
\hline$\underline{8}$ & Timbering Plan & & & \\
\hline & $\begin{array}{l}\text { Mobile Roof } \\
\text { Support Plan }\end{array}$ & & & \\
\hline 10 & Depth of Cover & & & \\
\hline 11 & Distance from & & & \\
\hline$\underline{12}$ & $\begin{array}{l}\text { ARMPS Pillar } \\
\text { Stability Factor }\end{array}$ & & & \\
\hline$\underline{13}$ & Ventilation & & & \\
\hline 14 & \begin{tabular}{|l|l} 
Methane \\
iberation \\
\end{tabular} & & & \\
\hline$\underline{15}$ & & & & \\
\hline 16 & & & & \\
\hline 17 & & & & \\
\hline 18 & & & & \\
\hline
\end{tabular}

Figure 4.9. Pre-Mining Risk Matrix

This final pre-mining matrix is developed in a manner which should enable a knowledgeable observer to record a well-documented perspective of the obstacles that are encountered on the pillaring section. To effectively use this form, it is necessary that the user understand pillaring risk factors and their effects on the pillaring section, 
and should be able to identify any mine-specific risk factors and incorporate them into the risk matrix. To use this form, one needs to address each risk factor, identify its presence or absence, or whether it is sufficient or insufficient. The second part of the form requires the use of qualitative judgment related to the likelihood of negative consequences. This qualitative assessment of risk level requires expertise and should be done only by knowledgeable individuals. This form is designed to identify and qualitatively assess risk so that risk factors can be addressed in the order of importance related to probable consequences. It is not meant to measure or assign quantitative risk but simply to identify risk factors and provide managers and mine planners with a method to focus their attention. This form could be completed by the foreman and crew at a pre-shift meeting and would aid in focusing attention on the risk factors that are likely to be encountered during the shift. It is also applicable to mine planning prior to pillar extraction.

Taking action to control risk prior to a loss or an incident is always preferable to reactive risk controls; therefore, this type of pre-mining risk matrix can be beneficial to both safety and production, and is flexible enough to be widely applicable, not only during pillar extraction but also in any other form of mining activity.

\section{Unsafe Performance Observation Form}

The unsafe performance observation form is designed to record the frequency of unsafe performances. The observation form changed very little from its original design. The final version (Figure 4.10) has blank sections built into the performance categories to permit expansion of categories. 


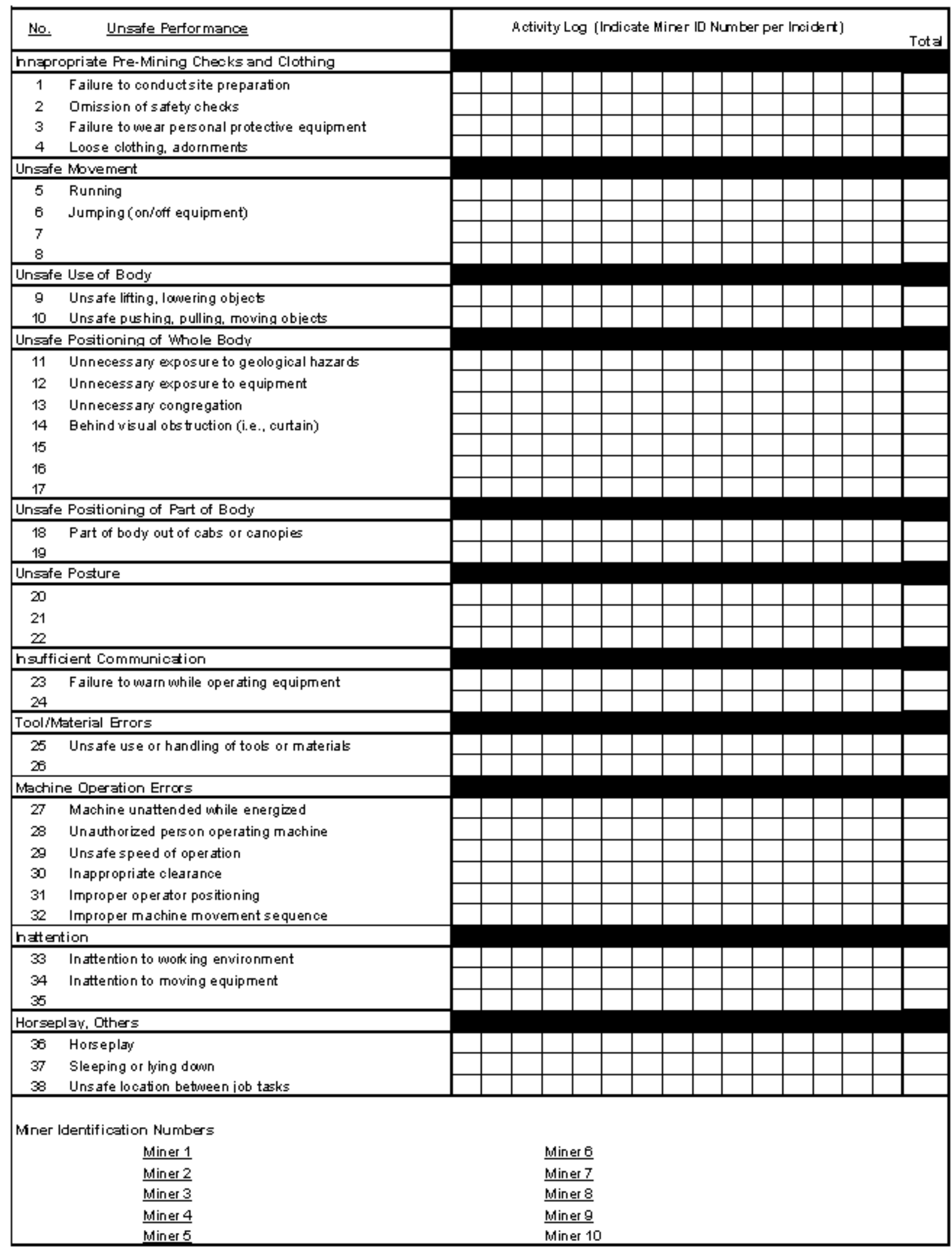

Figure 4.10. Unsafe Performance Observation Form 
Thus, the addition of the blank sections enables the observer to make on-the-spot additions to the form while underground. This is important during field application since it is impossible to predict all unsafe performances that will be encountered from mine-tomine or even day-to-day on the section. The form only requires familiarization with the form organization and the unsafe performances described. Once the observer knows what to look for, the technique only requires that the observer should be positioned to maximize visibility and minimize impact on the normal function of the crew.

Once in place, the observer must assign a number for each member of the crew. When an unsafe performance is to be recorded, the observer notes the number of the corresponding miner to the right of the observed unsafe performance. The results of this observation provides quantitative information with regard to the unsafe performance of the miners who were present in the active mining zone.

Due to the influence of boredom and fatigue on worker performance, it is important to observe for at least the duration of the mineworkers shift in order to gather accurate information pertaining to unsafe performance. If an observation must be completed incrementally, it is important that these increments are conducted at various times throughout the typical working shift and do not target only one specific part of the shift.

\section{Activity and Location Monitoring Form}

The initial strategy utilized three forms and three separate area division systems to record the position, timing and activities of miners on the section. However, attempting to accurately utilize three different systems is difficult in an ideal setting; performing these observations would likely prove to be very difficult when placed in the environment of an active pillaring section,. Therefore, in order to improve the applicability of the strategy, a condensed Activity and Location Monitoring form was developed. The final strategy utilizes the best aspects of the previous three forms, but facilitates its usage by confining the observation to just the active mining zone, and allows for the observation of all personnel within the active mining zone concurrently. The strategy is designed to record miner position, miner activity, and duration of positioning and activity. The form is set up to be used on a cut-by-cut basis and is shown in Figure 4.11. 


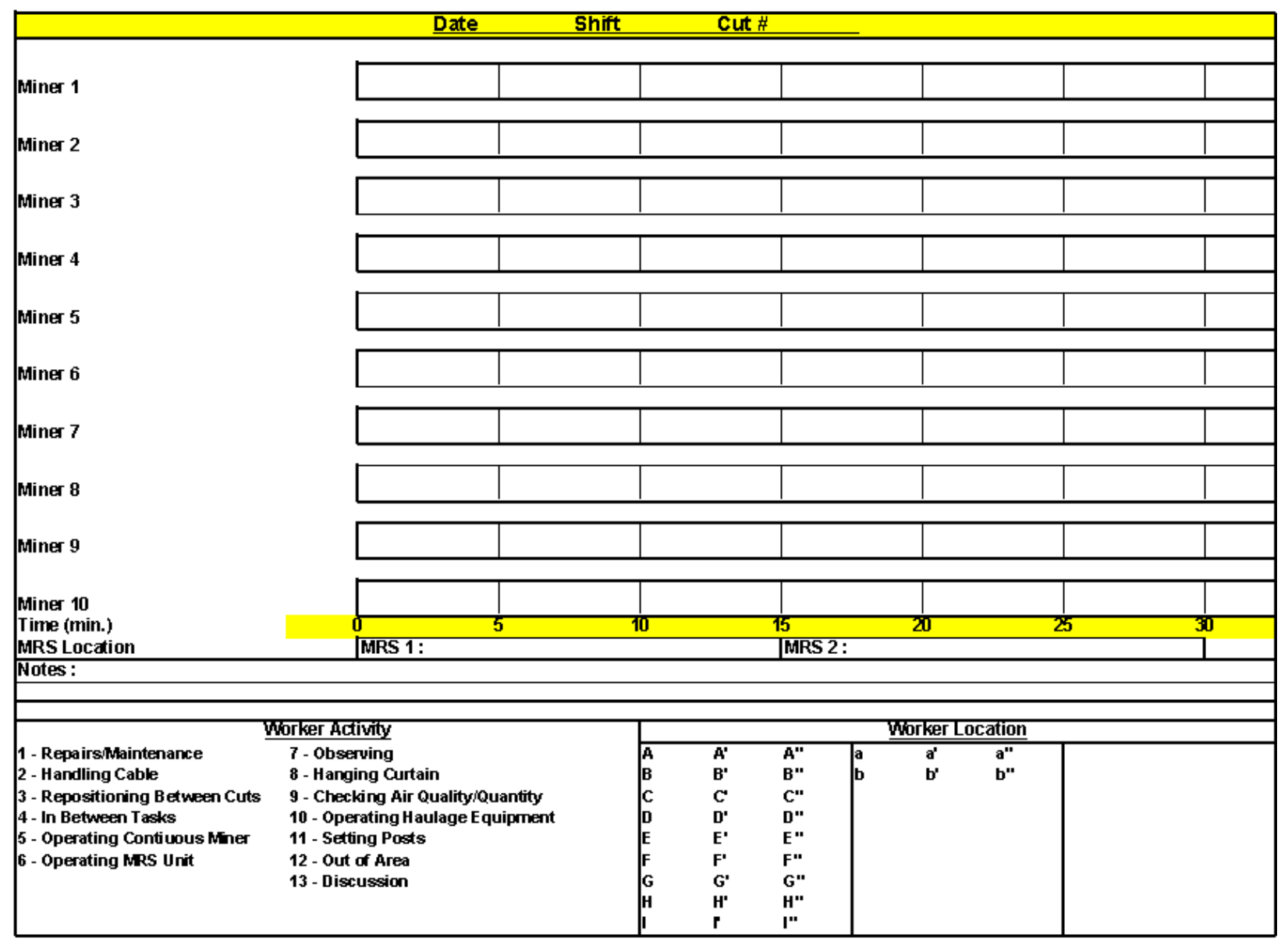

Figure 4.11. Activity and Location Monitoring Form

Prior to the observation, every miner on the section is assigned a row on the observation form. As previously stated, this form is designed to be utilized cut-by-cut; as each new cut is started, a new form should be used. The zero mark on the timeline represents the beginning of a new cut. The initial location and activity of every person in the active mining zone is recorded to the right of the zero indicator mark as cutting begins. The location is designated using the letter for the correct zone of occupation and the activity is logged using the appropriate numerical code. The activity and position code should be written one above the other within the timeline bar and that procedure should remain consistent throughout the observation. If the miner's position or activity changes, a line is to be drawn vertically through the timeline bar, indicating a stopping point for the previous location and activity and the beginning of a new location and activity. By delineating the timeline bar and logging position and activity in between, the observer can accurately record very detailed information using this sampling strategy. An example of the recording method is shown in Figure 4.12. 


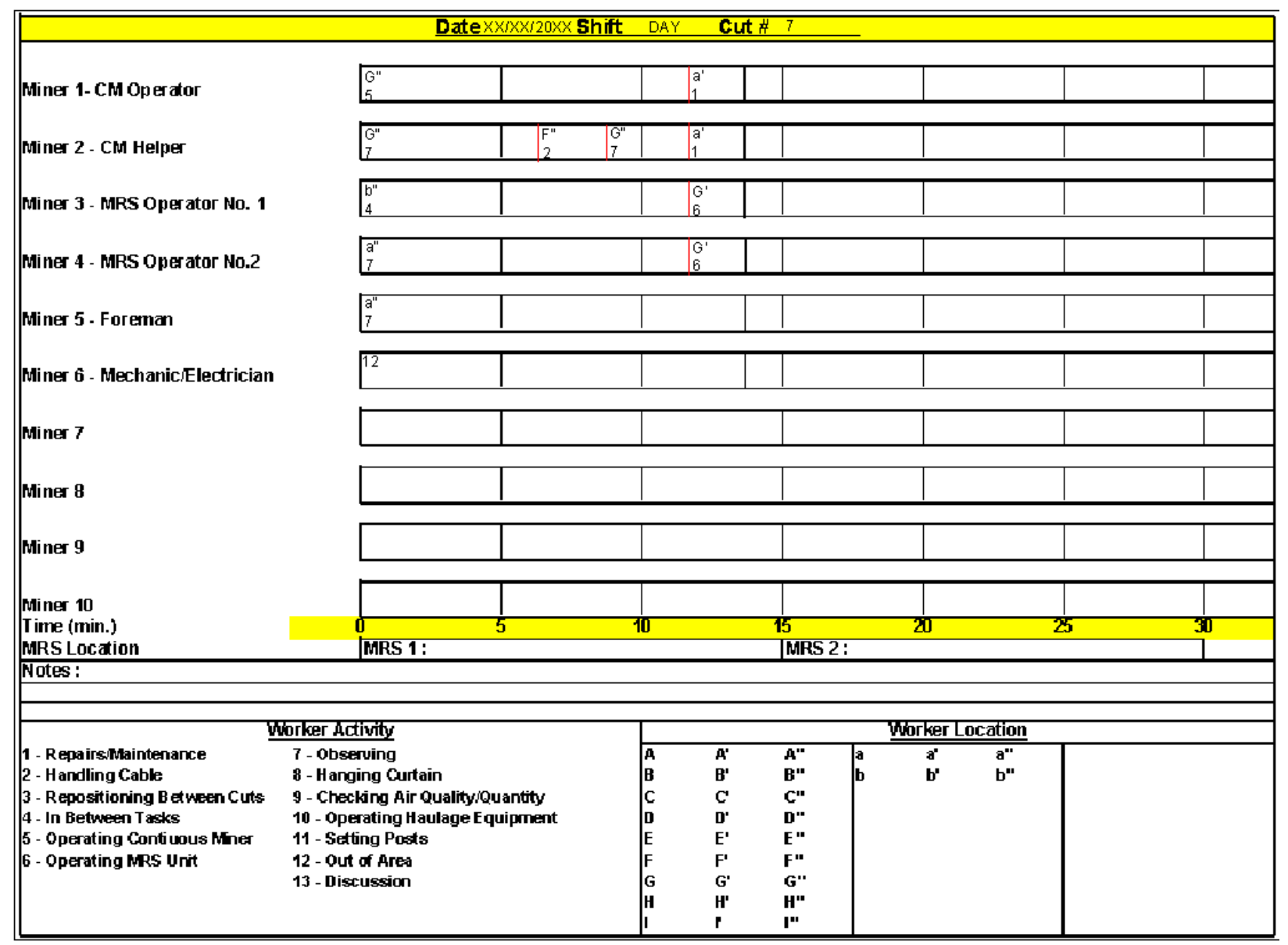

Figure 4.12. Example of Activity and Location Monitoring Form

In order to record miner location, it is necessary to divide the active mining zone into smaller areas that can be identified on the observation form. While it is impossible to design a sampling form that is suited to every cut sequence and mining conditions, some basic examples have been developed that can be applied to most situations and are demonstrated in Figures 4.13 and 4.14. 


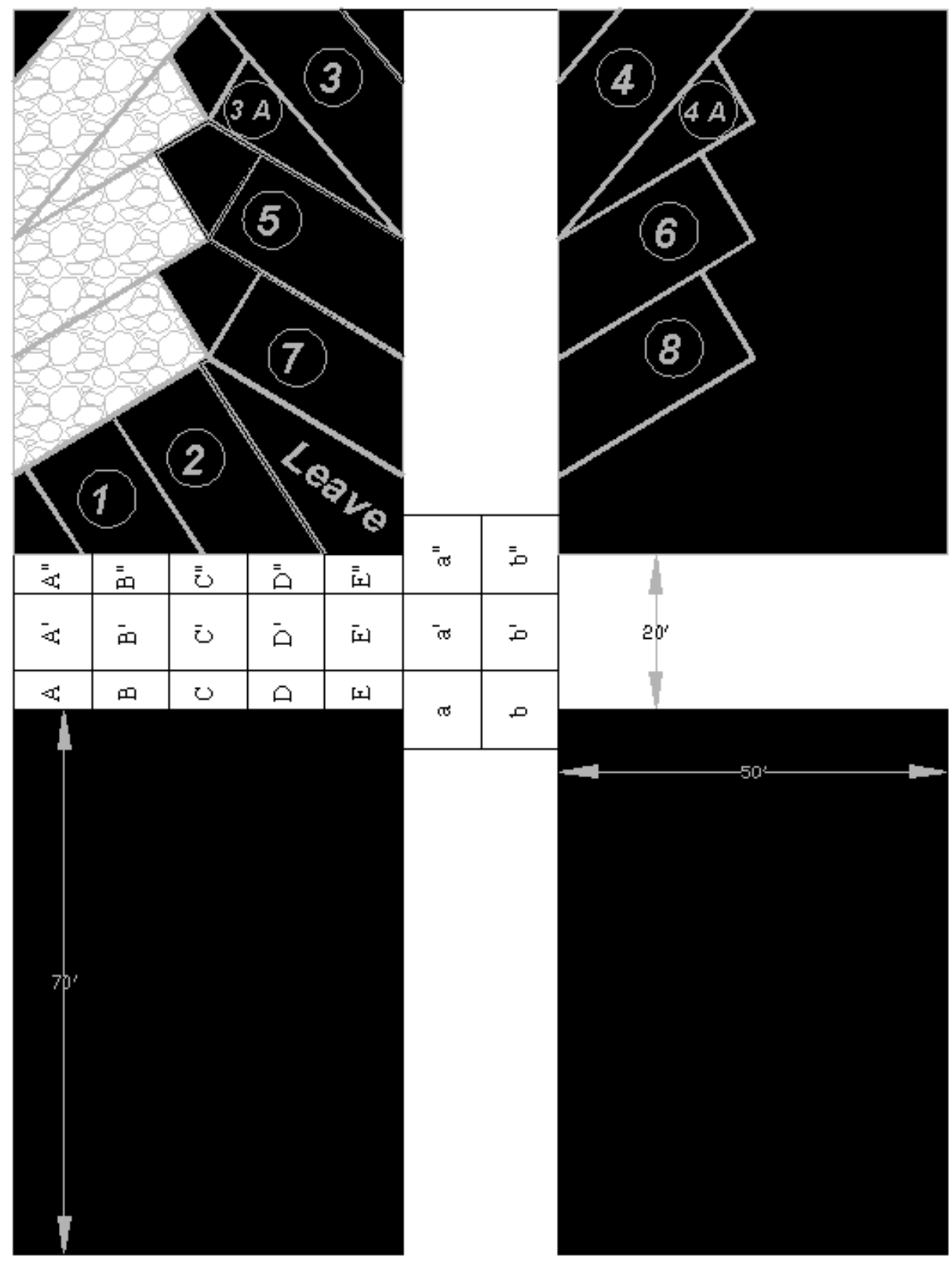

Figure 4.13. Area Division Example No. 1 


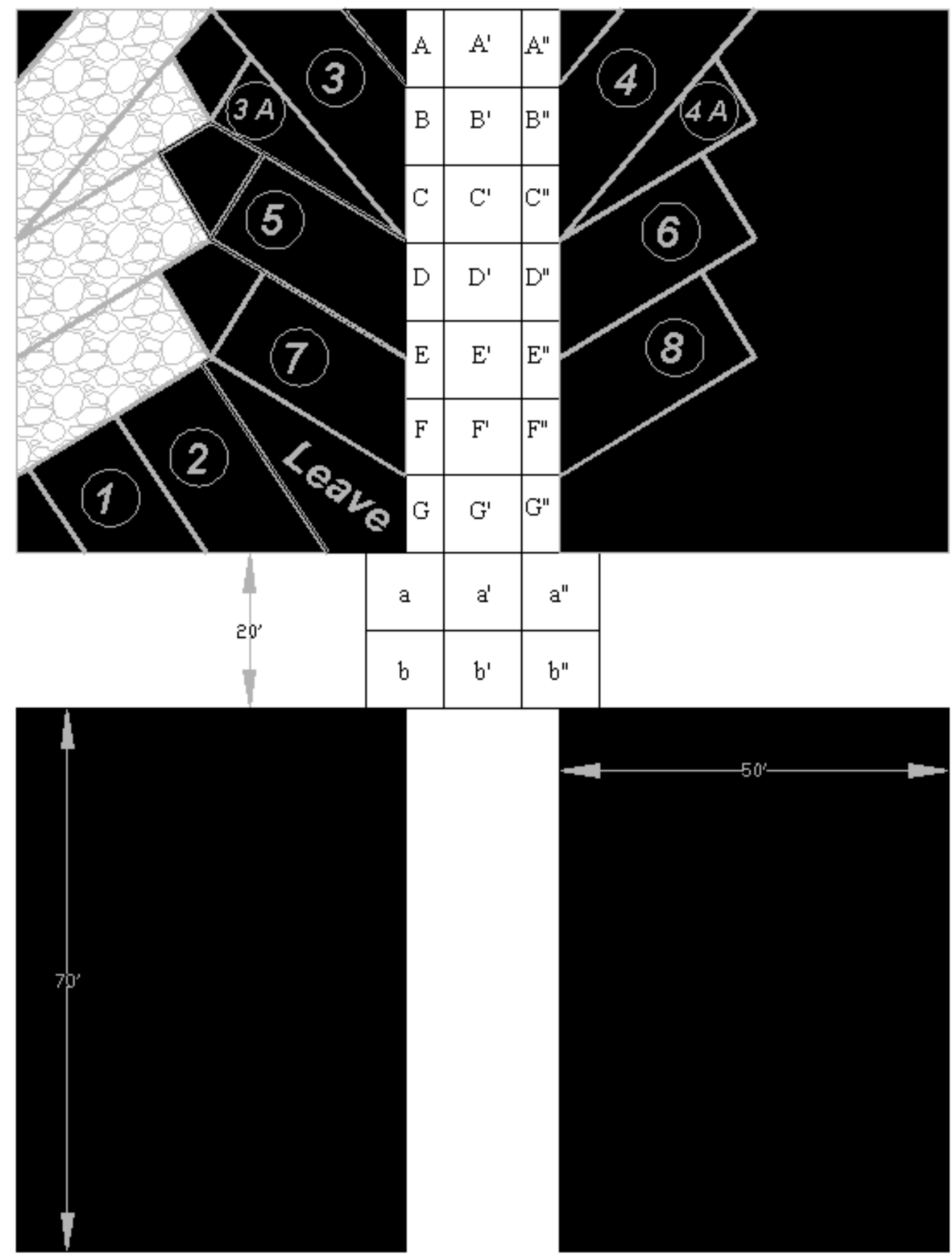

Figure 4.14. Area Division Example No. 2

Figure 4.13 represents an example of an area division for taking initial cuts when extracting pillars utilizing the Christmas-tree cut sequence. Notice that cuts 1 and 2 are both on the same side of the crosscut. These first two cuts are taken by the same technique that would be used in an Outside-lift cut sequence. Therefore, this example illustrates the zone division for an Outside-lift extraction, or initial same-side cuts on a Christmas-tree extraction. Figure 4.14 represents typical main cuts made during a 
Christmas-tree pillar extraction. The zone division is the same as shown in Figure 4.13, except that the zones are oriented down the entry as opposed to the crosscut.

The zones are recorded alphabetically and prime and double prime designations are utilized as before. The zone nearest the gob should be labeled by an uppercase letter " $A$ " and each subsequent zone progressing toward the intersection will be labeled with the following letter of the alphabet. Once the intersection is reached, the letters restart at the beginning of the alphabet but are designated by lowercase. The prime and double prime are for designation from left to right. A letter without a prime or double prime designation should always be on the left side of an entry or crosscut, or the left portion of the intersection while facing towards the active face. A single prime letter always represents the middle of an entry, crosscut or intersection. A double primed letter indicates the right side of an entry crosscut or intersection. By maintaining this style of nomenclature, the observer can identify and record zones while minimizing the need to reference the zone division drawing. After some practice, an observer should be able to visually identify the distance from a reference point, whether it is the cut being taken or the corner of the pillar, and accurately identify the occupied zone and record the proper information.

As shown in the zone division examples, the zones in the entries and crosscuts are not uniform in size from left to right. This decision was made because haulage vehicles typically operated in the center of the entry or crosscuts, and if all zones were equal in width, the haulage would be occupying potentially two or more at a time and it would be difficult to determine if mine personnel were impeding or unnecessarily exposed to the hazard of the moving equipment. Based on this, it was decided that the middle zone would be twice the width of the left and right zones. For example, if an entry is $20 \mathrm{ft}$ wide, the middle zone will be $10 \mathrm{ft}$ wide while the zones closest to the coal ribs are $5 \mathrm{ft}$ wide. This enables the observer to accurately indicate if a miner were to be standing tight up against the rib to avoid machinery or if the miner happened to be out towards the middle of the entry. The length of all zones in the crosscuts or entries was set at $10 \mathrm{ft}$. Anything smaller than $10 \mathrm{ft}$ makes the estimation of the occupied zone much more difficult. The intersection is divided into six zones; all are identical in length and width and actually protrude slightly into the crosscut or entry, depending on where mining is occurring. The zones are all ten feet long and each zone's width is one half the entry width. By extending the zones slightly outside the intersection, it becomes possible to record the presence of any observers that occasionally place themselves just barely out of the active intersection. It is correct that they are technically not in the intersection, but their extremely close proximity does not really provide them any protection from the hazards of the active intersection, so by extending the zones to occupy these favored vantage points, an accurate measure of hazard-exposure can be recorded. Using the cut sequences and pillar dimensions at any given mine, an area division map such as in Figures 4.13 and 4.14, can be designed and used in conjunction with the Task and Location Monitoring form.

The major strength of this method of zone or area division is its simplicity and flexibility; it is applicable to every pillaring section and can be effectively modified as needed. If an observer notices an area that is experiencing high occupation exposure time or was located particularly close to some known hazard, any number of new observation zones can be added to the form. By designing a sampling strategy that is 
rigid enough to standardize the type of information gathered, yet flexible enough to adapt to changing scenarios underground, an effective performance sampling can be conducted and the benefit to mine safety and training should be appreciable.

\section{Summary}

Performing these observations, admittedly, is time consuming, possibly taking one individual a week or more to complete them all. The time spent processing the data will likely take just as long, if not longer. However, when everything is completed, safety managers will have a powerful tool to combat risk. In fact, a safety manager should be able to identify trends in the data that suggest problems. Some problems may require immediate intervention. Other problems may be addressed during routine training sessions. Regardless of how the issues are addressed, the key is to identify problems through analysis of the gathered information and provide proper intervention before unsafe performances result in accidents. This should provide the most efficient and cost-effective method to control the residual risks remaining from the shortcomings of engineering risk controls. 


\section{CHAPTER 5. APPLICATION OF THE WORKPLACE SAMPLING STRATEGY FOR PILLARING SAFETY}

\section{Introduction}

In order for any strategy or process to have an influence on safety and training, it must be applicable to the environment for which it was designed to influence. To prove the applicability of the performance sampling strategy, it was necessary to test the strategy on pillaring sections in Central Appalachia. The goal of the field applications testing was to utilize the sampling strategy to gather information and assess the ability of an observer to perform the sampling strategy on a pillaring section. Although, the value of this type of observation is obvious, it is necessary to also process and evaluate the information gathered in order to provide examples.

Two field applications were conducted. Both mines were located in Central Appalachia. Field testing was conducted over a period of six days. Detailed results have been provided for the mine described as Mine No.1. The field application at Mine No. 2 demonstrated a very similar level of success as Mine No.1. Due to the similarities, a description of the application will be provided, but lengthy detailed results have been omitted. Original forms utilized during the field applications are provided in Appendix C.

\section{Mine No. 1 Site Description}

Mine No.1 is located in Central Appalachia. At the time of the field application, mining height varied between five and six feet. It has been overmined in six separate seams, with the closest being $60 \mathrm{ft}$ above the active seam, and has been undermined by one seam 100 feet below the active seam. The immediate roof consists of approximately 60 inches of shale or sandstone (depending on location within the mine) and often contains coal streaks that weaken the structural integrity of the roof and proved to be problematic during the time of the observation. The bolting pattern utilizes a 4-ft-by-4-ft pattern employing 48-inch roof bolts. The section contains a Joy Model $14 \mathrm{CM}$ continuous miner, a scoop, three battery-powered coal haulers, and four 800-ton Fletcher mobile roof supports. The mine operates three shifts per day (two production, one maintenance), five days per week. Each operating section is staffed by a crew of 12-13 miners; however only four to five were consistently in the active mining zone. Minimum pillar dimensions permitted by the roof control plan are 50 feet wide and 70 feet long. Each entry and crosscut is 20 feet wide and annual production is estimated to be approximately one million clean tons. During the observation, pillars were being extracted from under approximately 1000 feet of cover. The age of the pillars being mined during the observation was approximately 9 to 10 years. Due to the age of the pillars and associated stress caused by a combination of deep cover, and interactions from over and undermining, pillars had experienced significant degradation and, in most places, timber posts had to be set alongside the coal ribs to maintain appropriate entry dimensions. Supplemental roof and rib bolting were also utilized to facilitate roof support and prevent rib spalling. The field application of the performance sampling strategy took place over three days for the entire dayshift each day. The first day was used to 
familiarize the observer with the personnel and the mine as well as to complete the premining risk matrix. The worker performance observation was conducted on the second day and the task and location monitoring observation was conducted on the third day.

\section{Pre-Mining Risk Matrix}

Based upon discussions with safety personnel and the section foreman, as well as a review of the roof control plan, the pre-mining risk matrix shown previously in Figure 4.9 was completed and proved to be a good starting point for drawing awareness to the mining conditions associated with this operation. In particular, it helped to highlight the lack of uniformity of understanding of mining conditions among various personnel. It became necessary to consult multiple individuals to gather sufficient information to complete the form, indicating a lack of awareness. It is this lack of awareness among management personnel that the pre-mining risk matrix is aimed at eliminating. If managers are required to meet as a group and complete this pre-mining risk matrix, all members that participate should be equally aware of the hazards and potential risks with regard to their mining operation. This could aid in reducing management misunderstanding and improve safety.

\section{Unsafe Performance Identification}

After completion of the physical risk matrix, a general knowledge of the mine's characteristic hazards and how those hazards may affect the miners is obtained; attention is then directed toward the performance and behavior of the miners on the section. The first step in sampling miner performance is to observe the operation of the crew while utilizing the unsafe performance identification form. The information recorded in the unsafe performance identification form was collected over a period of one day, during dayshift. As shown in Figure 5.1, numerical designations were assigned to specific individuals or job titles and, as each unsafe performance was observed, the number of the offender was recorded to the right of the corresponding unsafe performance. The form proved to be effective for recording the information. However, one difficulty can develop when the observer in an attempts to keep the form and its contents secret. Taking notes of this nature can make miners feel uncomfortable and can lead to uncooperative responses from them. However, once miners understood that the observation form was safety oriented and was in no way intended to be a tool to justify any punitive measures, most were very cooperative. However, the completed forms were kept in the observer's possession at all times, and the results were never revealed to anyone on the section. The results of the observation have been condensed into one form shown in Figure 5.1. 


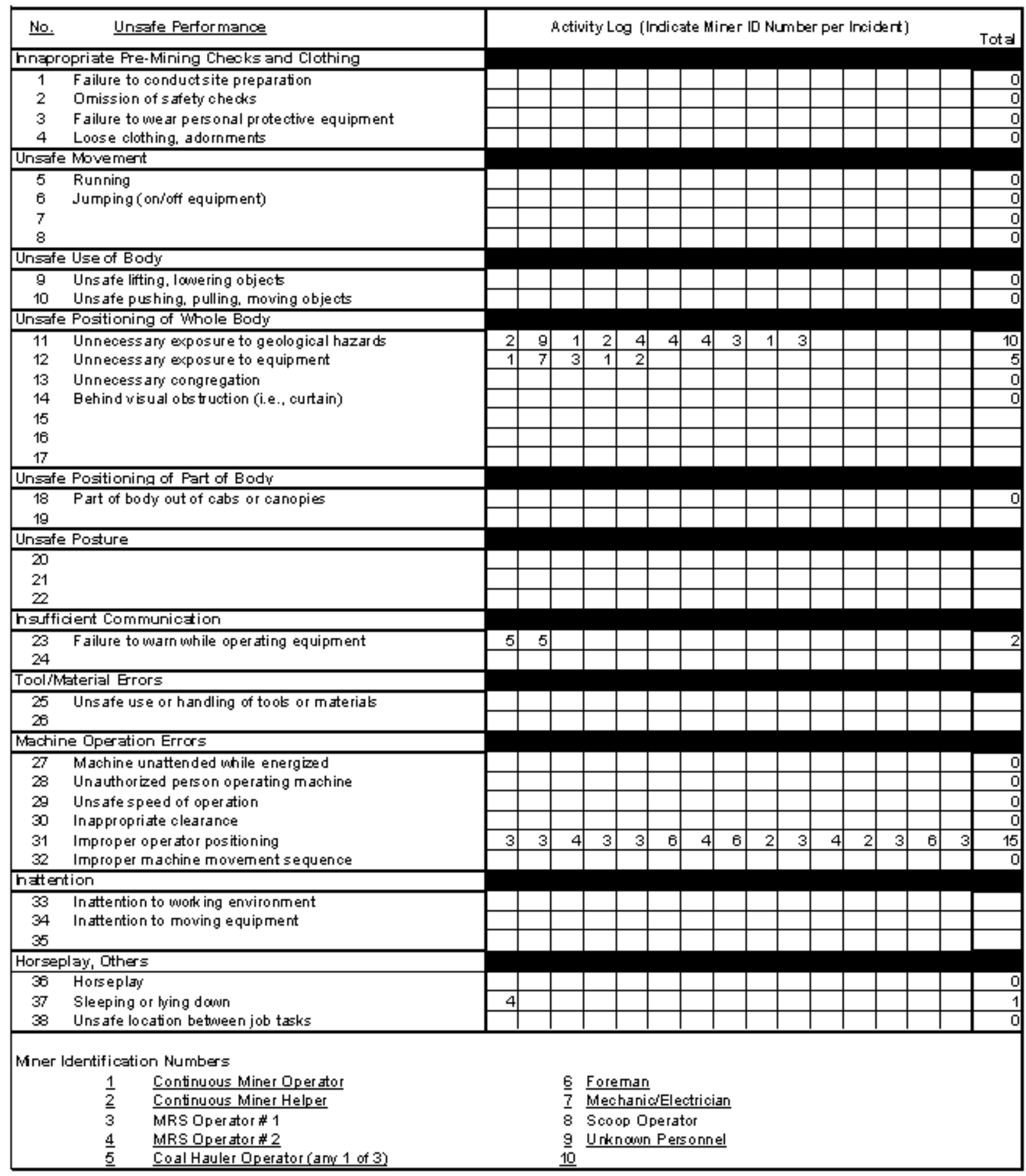

Figure 5.1. Completed Unsafe Performance Observation Form for Mine No. 1 


\section{Results from Sampling at Mine No. 1}

Understanding the results of a workplace sampling is critical to effectively making decisions based on the observations. One effective method of conveying the results to others is through graphical representation. The graphs can be useful for identifying problems and can also be used for establishing concrete justifications for safetyperformance topics during safety training. The results of the unsafe performance observation form are shown in Figures 5.2 to 5.6.

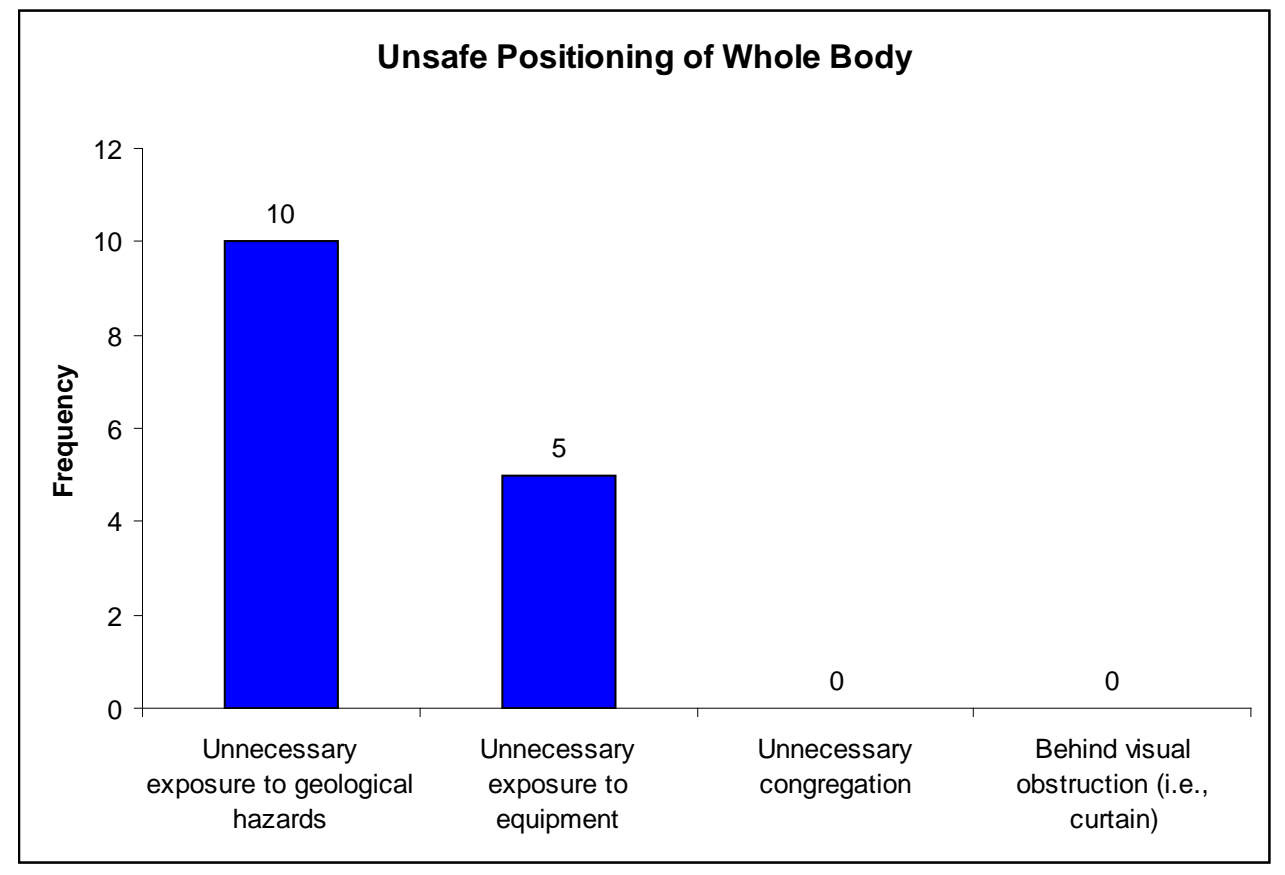

Figure 5.2. Unsafe Positioning of Whole Body

Figure 5.2 shows that there were fifteen observed occurrences of unsafe positioning of the whole body, falling into two categories, "Unnecessary exposure to geological hazards" and "Unnecessary exposure to equipment". In this observation, unnecessary exposure to geological hazards typically consisted of moving inby the miner. Unnecessary exposure to equipment consisted of miners moving through known machinery pinch points while the machine was energized, or placing themselves in a position where they obstructed haulage and were at higher risk for being struck by moving equipment.

Figure 5.3 demonstrates that, during this observation, two coal-hauler operators failed to warn while operating their machines. In this situation, both performances consisted of operators failing to give audible signals before proceeding through ventilation curtain. 


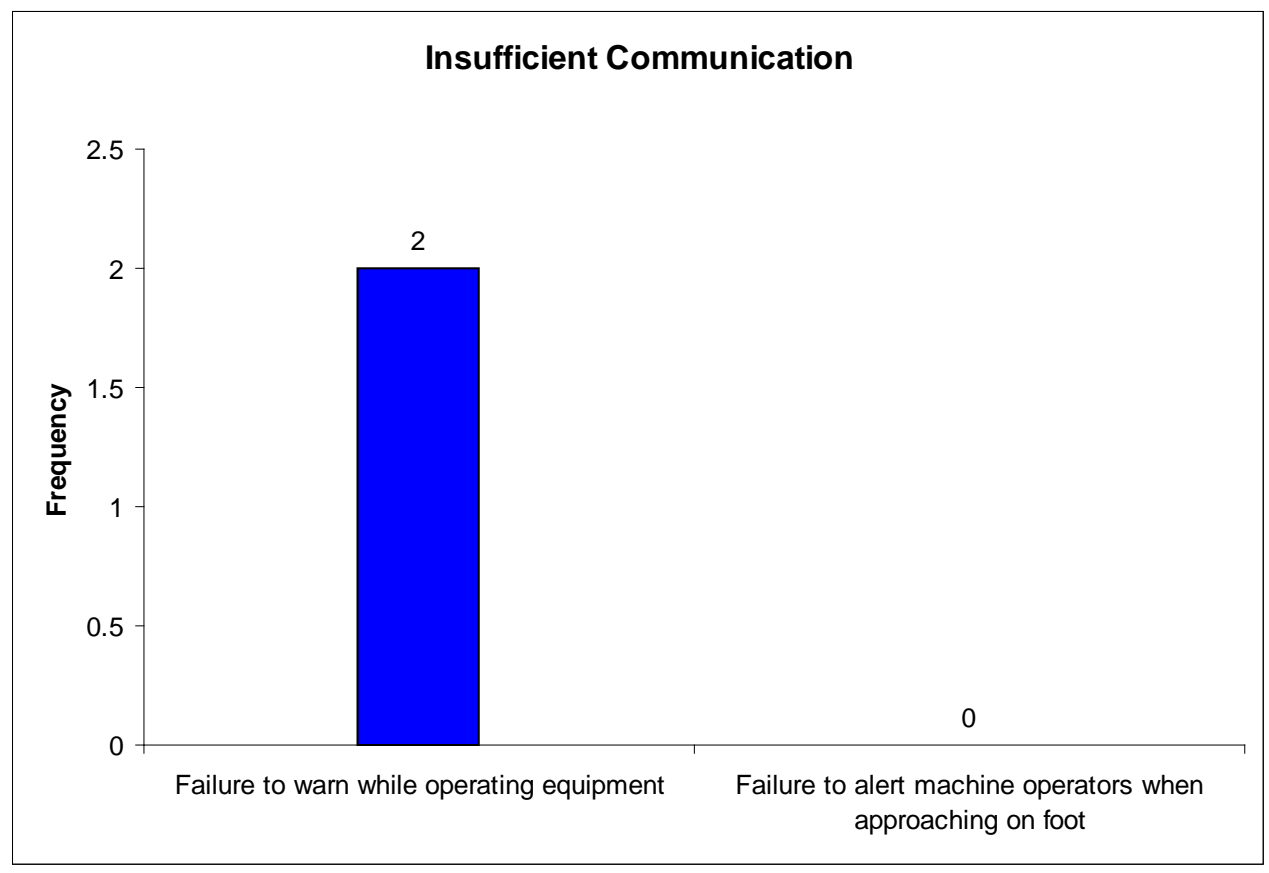

Figure 5.3. Insufficient Communication

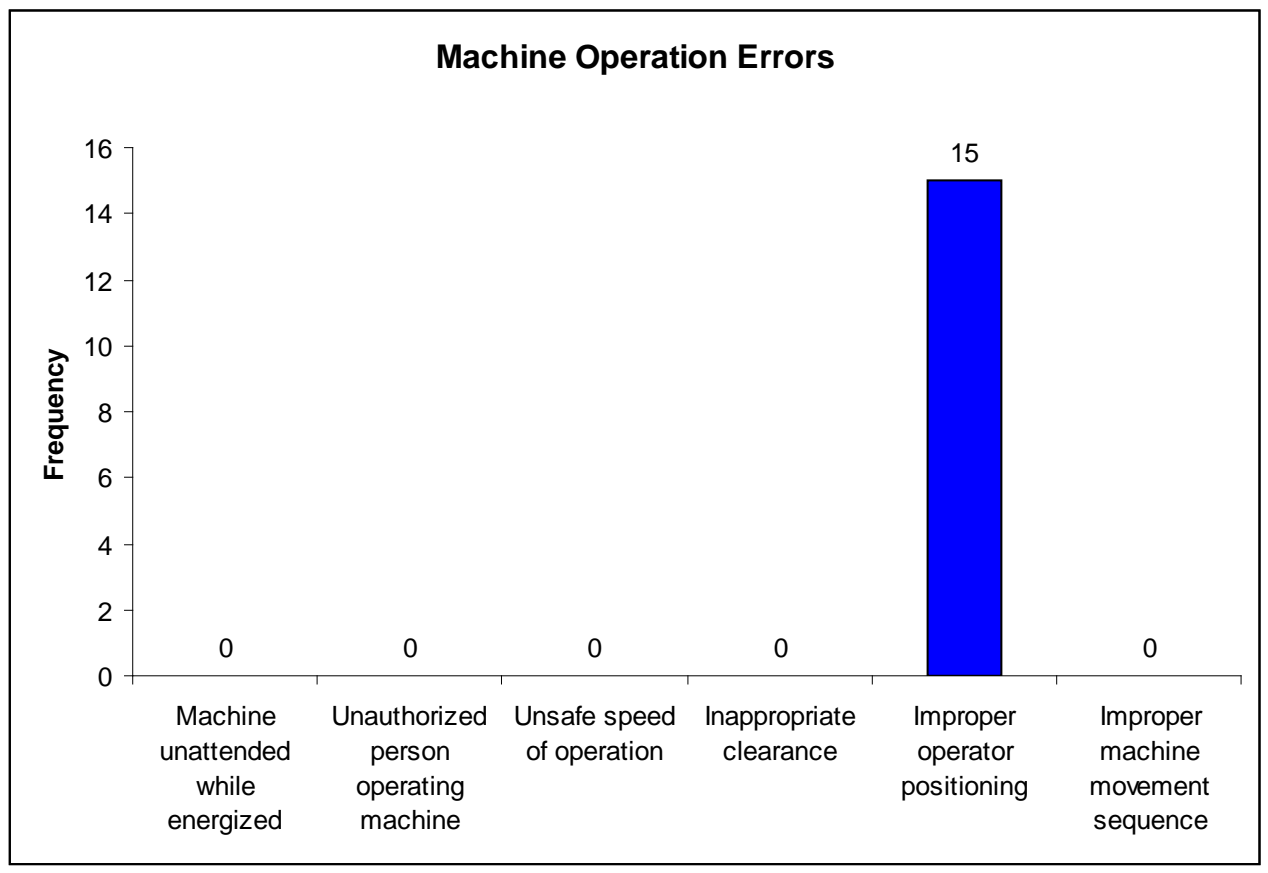

Figure 5.4. Machine Operation Errors

Figure 5.4 represents the frequency of machine operation errors. There were fifteen observed occurrences of poor operator positioning. By referring back to Figure 5.1, it is shown that MRS operators and miners operating outside their typical duties were most prone to improper positioning while operating machinery. 


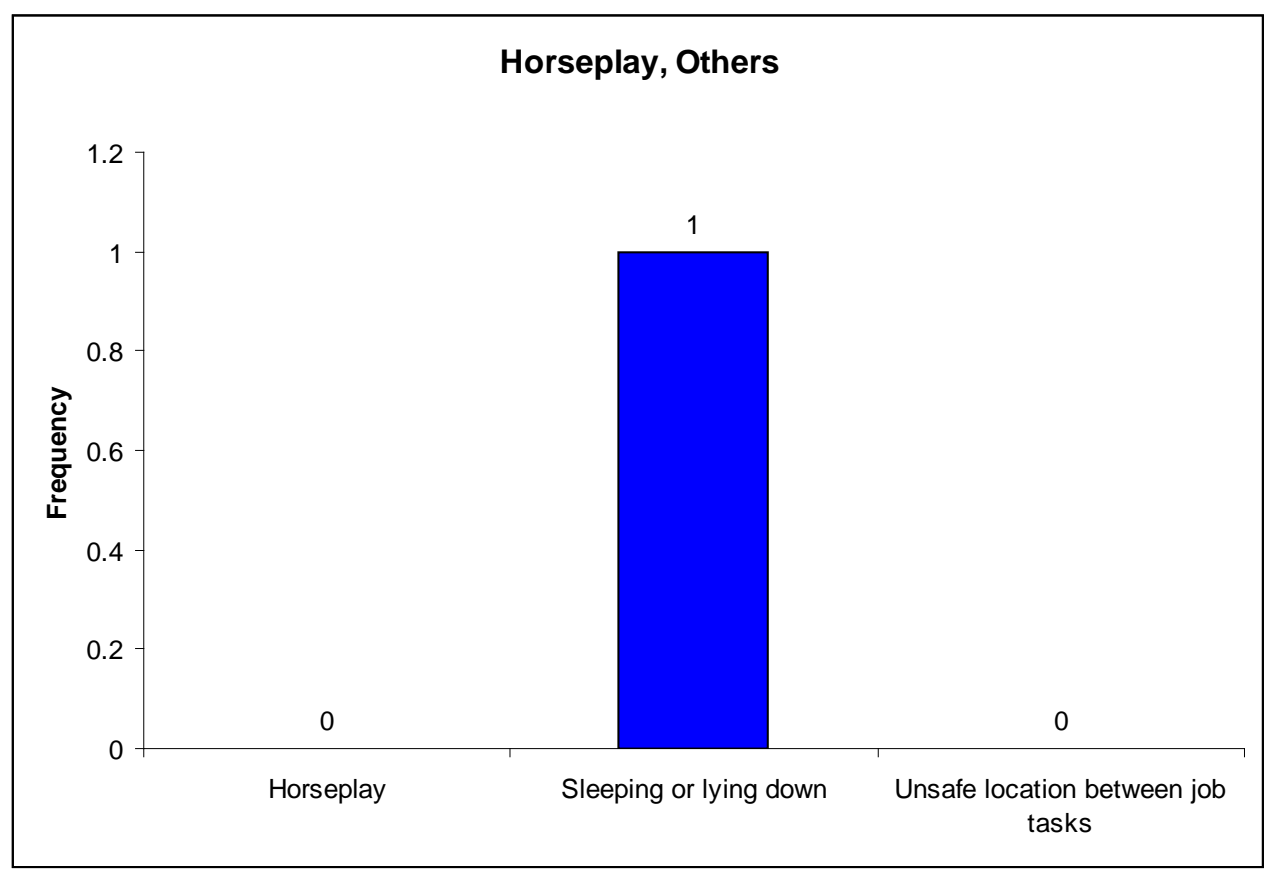

Figure 5.5. Horseplay, Others

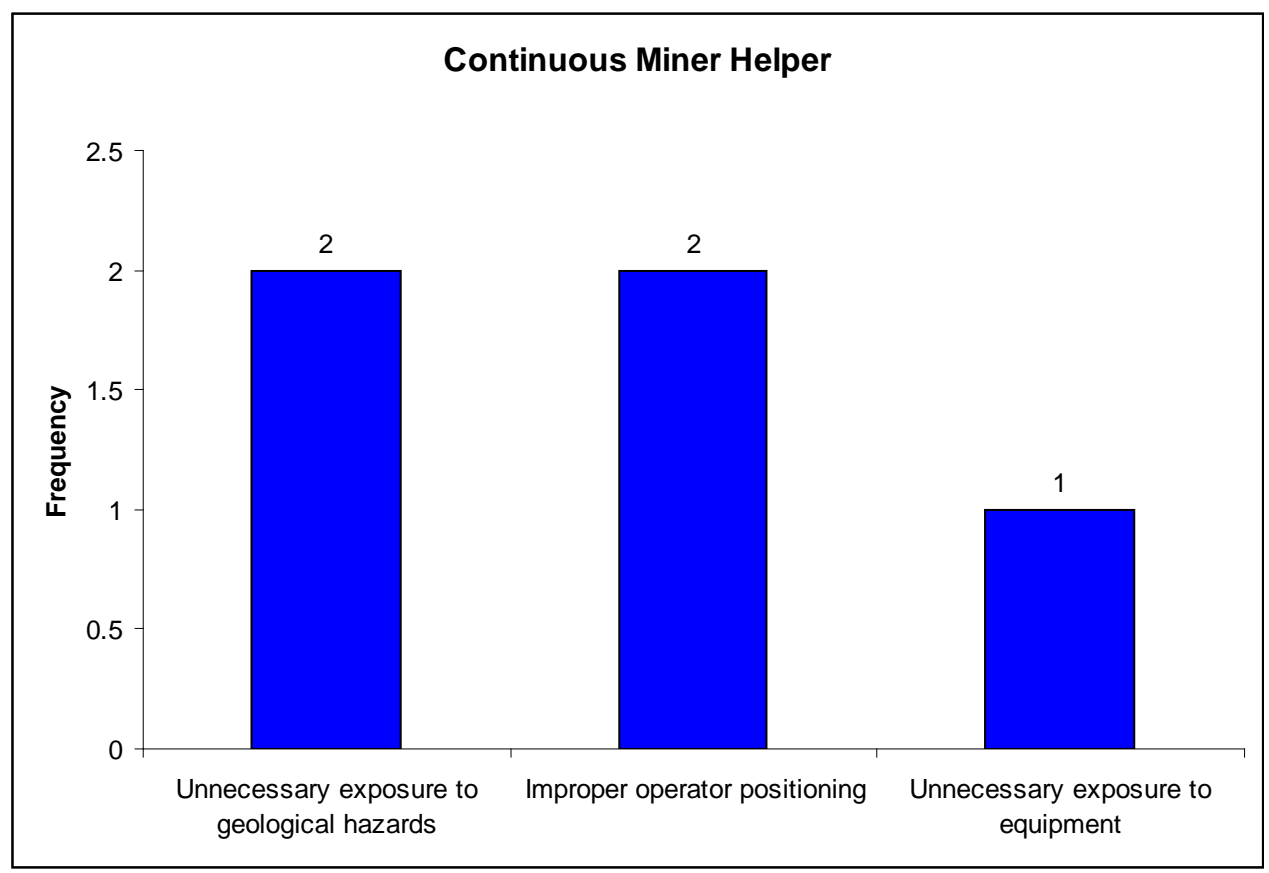

Figure 5.6. Continuous-Miner-Helper Evaluation

Figure 5.6 demonstrates the utilization of the unsafe performance observation results to focus attention on an individual, in this case the continuous miner helper. The ability to equally assess a group as well as an individual with one observation is a significant strength of this observation strategy. By performing the Unsafe Performance 
Observation, safety managers can use the results directly to influence safety training as well as direct further observations, such as the Task and Location Monitoring Observation. The components of the strategy presented in this research were designed to be sequential and complementary to each other. Initially, the Pre-Mining Risk Matrix identifies general hazards and risk factors. Then, the Unsafe Performance Observation looks for specific miner performances that affect safety. Finally, the Activity and Location Monitoring Observation records highly specific information regarding the timing and location of activities and the identities of the miners performing the activities. The complementary nature of this strategy is clearly observed in the following section, by the identification of personnel positioning that corresponds directly to the unsafe performances observed using the relevant form.

It is important to also address the need to conduct the Unsafe Performance Observation, as well as the Activity and Location Monitoring Observation for an extended period of time, preferably the entire shift. During pillaring, several members of the crew, particularly MRS operators, find themselves with a significant amount of downtime between tasks. Boredom has been observed to be a factor in the positioning and activities during downtime. As the day progresses and boredom grows, the tendency of the miners is to migrate closer to the continuous miner and the face for a better vantage point. Due to the observed differences in performance from the beginning to the end of the shift, it is important observe, for at least, an entire shift in order to record quality information.

\section{Activity and Location Monitoring}

The field application of the Activity and Location Monitoring Observation form required an eight hour shift to complete. Observation was conducted on a cut-by-cut basis. Due to the large amount of information gathered, only the results from two cut observations are presented in this thesis. The cuts presented were taken from two adjacent pillars and are numbered according the sequence that the cut was mined at the beginning of the shift. These two cuts were selected for presentation due to the diversity of information that was gathered during their extraction.

\section{Cut No. 2}

Figure 5.7 shows the location of the cut being analyzed, as well as prior and future cuts and the location of the MRS units. The evening shift had previously mined the first four cuts and the day shift was charged with finishing the pillar extraction. After consultation with the section foreman, the drawing used by the observer was immediately modified to reflect the actual conditions and the updated cut numbers. After the drawing was modified, what previously would have been the sixth pillar cut was changed to the first, and so forth, for the last four cuts. Figure 5.7 reflects these changes and shows the previous extraction of the first cut of the day and the location of the second cut that is presented here. The vantage point chosen for this observation was the right rear portion of the zone designated b". This observation point was selected because it provided good visibility, was located as far from the gob area as 
possible and was not located in a haulage route. If the crosscut had been part of a haulage route, the alternate vantage point would have been in zone $b$. While zone $b$ is closer to the gob, it does offer a physical barrier (MRS units) and is out of the haulage route. Information was gathered from the time cutting began and continued until the MRS units had been repositioned for the next cut. The entire cut extraction, including MRS repositioning, took approximately forty-one minutes. Physical reference points such as the location of the pillar corners and roof bolt spacing were utilized to estimate distance and, therefore, identify the zones that were occupied during the cutting process. A tool, such as a laser distance measuring device, would yield more precise results, however this type of technology is currently not permissible inby the last open crosscut.

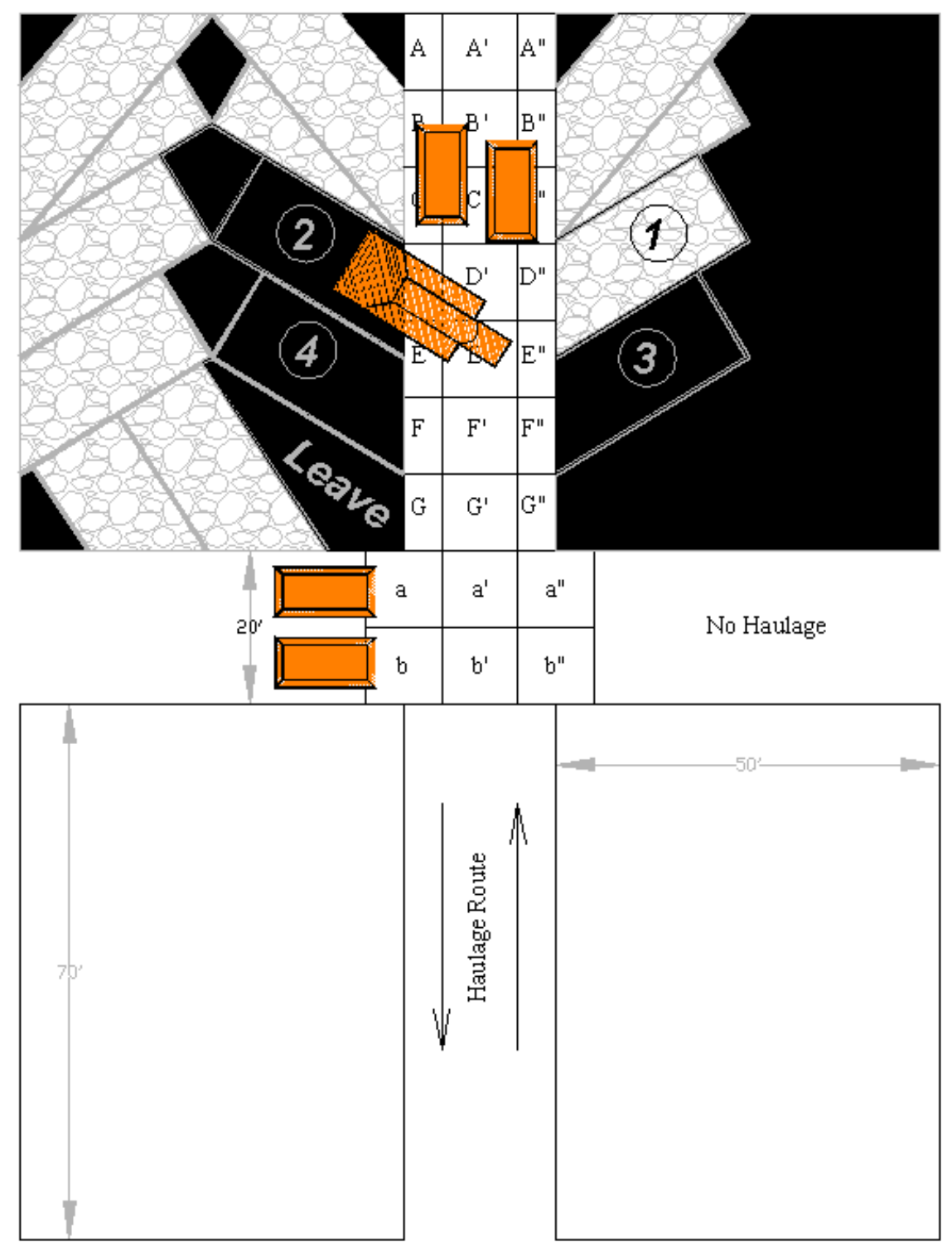

Figure 5.7. Area Division and Cut Numbering for Cut No. 2 
After the changes were made to the map, the Activity and Location Monitoring form, previously shown in Figure 4.11, was used to record time, position and activity of everyone that was present in any of the zones shown in Figure 5.7. The observation was conducted with minimal difficulties and proved to work as it was intended. The zone division, as well as the activity coding, were appropriate and accurate for the conditions encountered. After the observation was completed, the information was extracted from the form and represented in a graphical format. The original information for Cut No. 2 is provided in Appendix C.

Exposure to the Active Mining Zone. Pillaring hazards can be highly unpredictable due to the variable nature of the pillaring process and environment. While there are many engineering methods to control pillaring hazards, there is still residual risk that is beyond the current scope of engineering control. One means of reducing the residual risk is the reduction of hazard exposure. By reducing the amount of time personnel are exposed to a hazard or risk source, the risk posed by the hazard is decreased. In order to reduce hazard exposure, it is necessary to assess the level of exposure each miner encounters while performing their designated activities. By better understanding activity locations and times, managers can assess what hazard exposure is necessary to perform an activity and what exposure is unnecessary and can be reduced through training and intervention.

Figures 5.8 to 5.13 represents the time spent in designated zones by each member of the section crew. By comparing these zone occupation times with the Area Division Map shown in Figure 5.7, the appropriateness of each miner's location during the activity can be assessed. Using the Continuous Miner Helper as an example, the helper spent five minutes in zone D" during Cut No. 2. Figure 5.7 clearly shows that this is a dangerous location inby the miner and adjacent to the previous Cut No. 1. The Miner Helper should not have been performing any task in that area; therefore the helper's risk could be reduced by not venturing into such areas. The MRS operator designated as MRS Operator No.1, and the Mechanic/Electrician were out of the active mining zone during Cut No. 2. Blank graphs, labeled for MRS Operator No. 1 and the Mechanic / Electrician have been included to demonstrate that, typically, there would be results for both mineworkers if they had been present. 


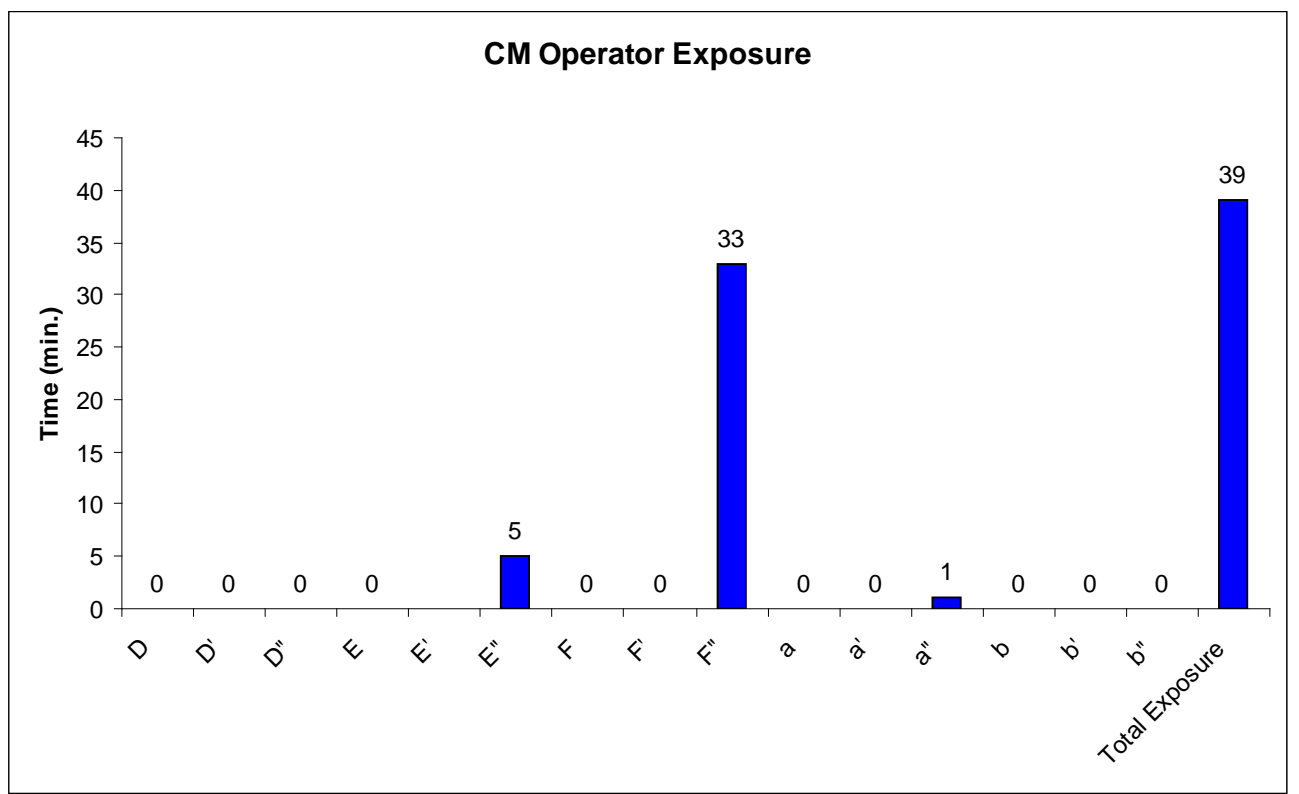

Figure 5.8. Continuous-Miner Operator Exposure

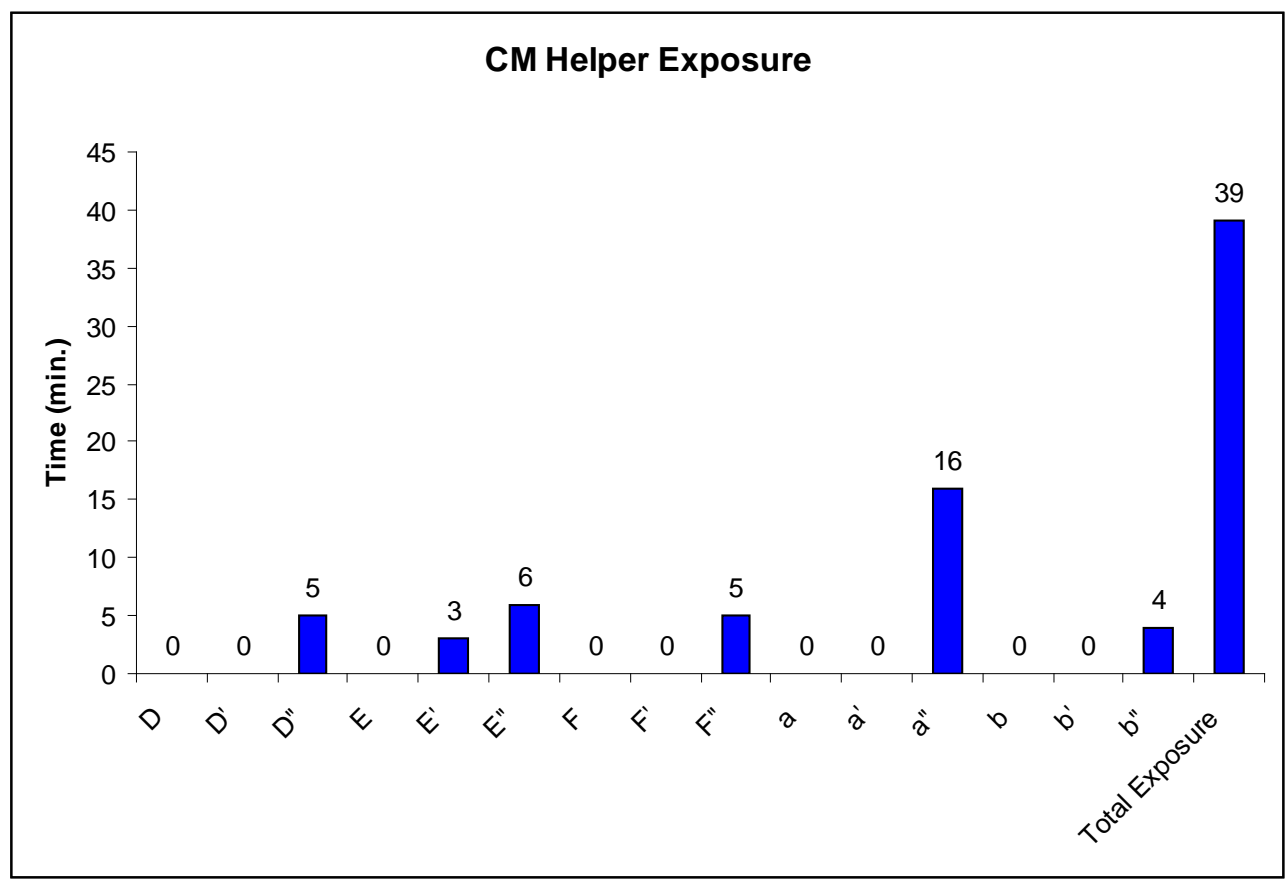

Figure 5.9. Continuous-Miner-Operator Helper Exposure 


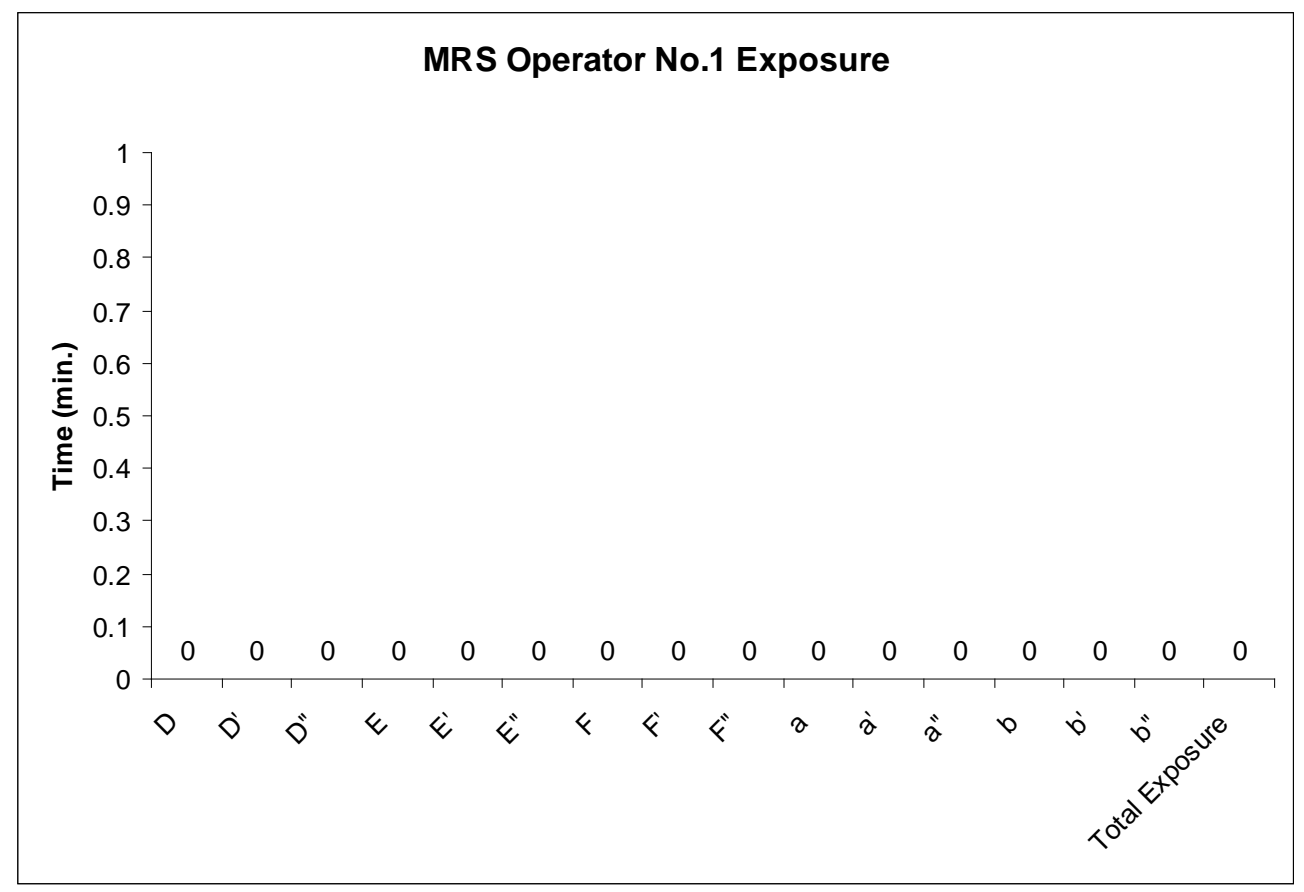

Figure 5.10. MRS Operator No.1 Exposure

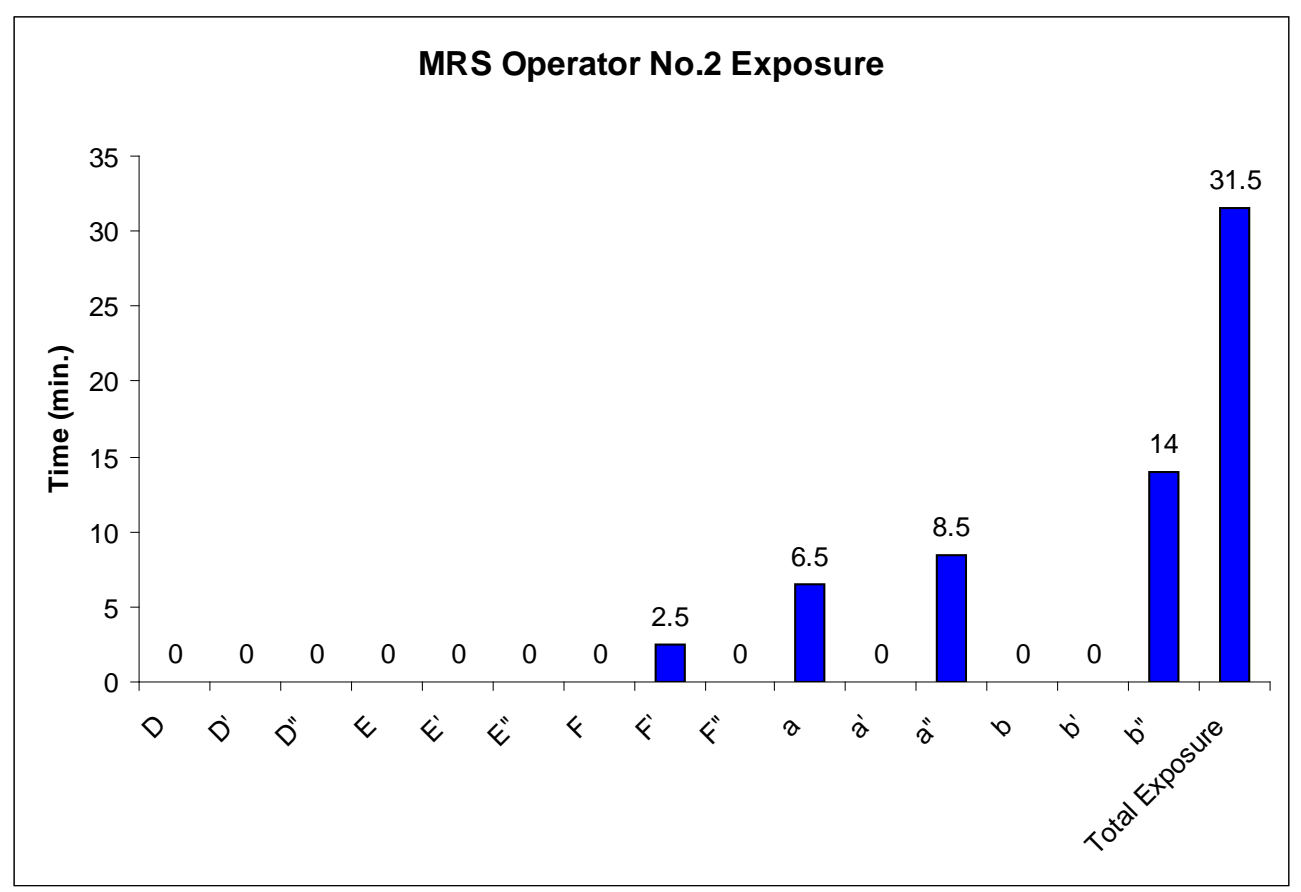

Figure 5.11. MRS Operator No. 2 Exposure 


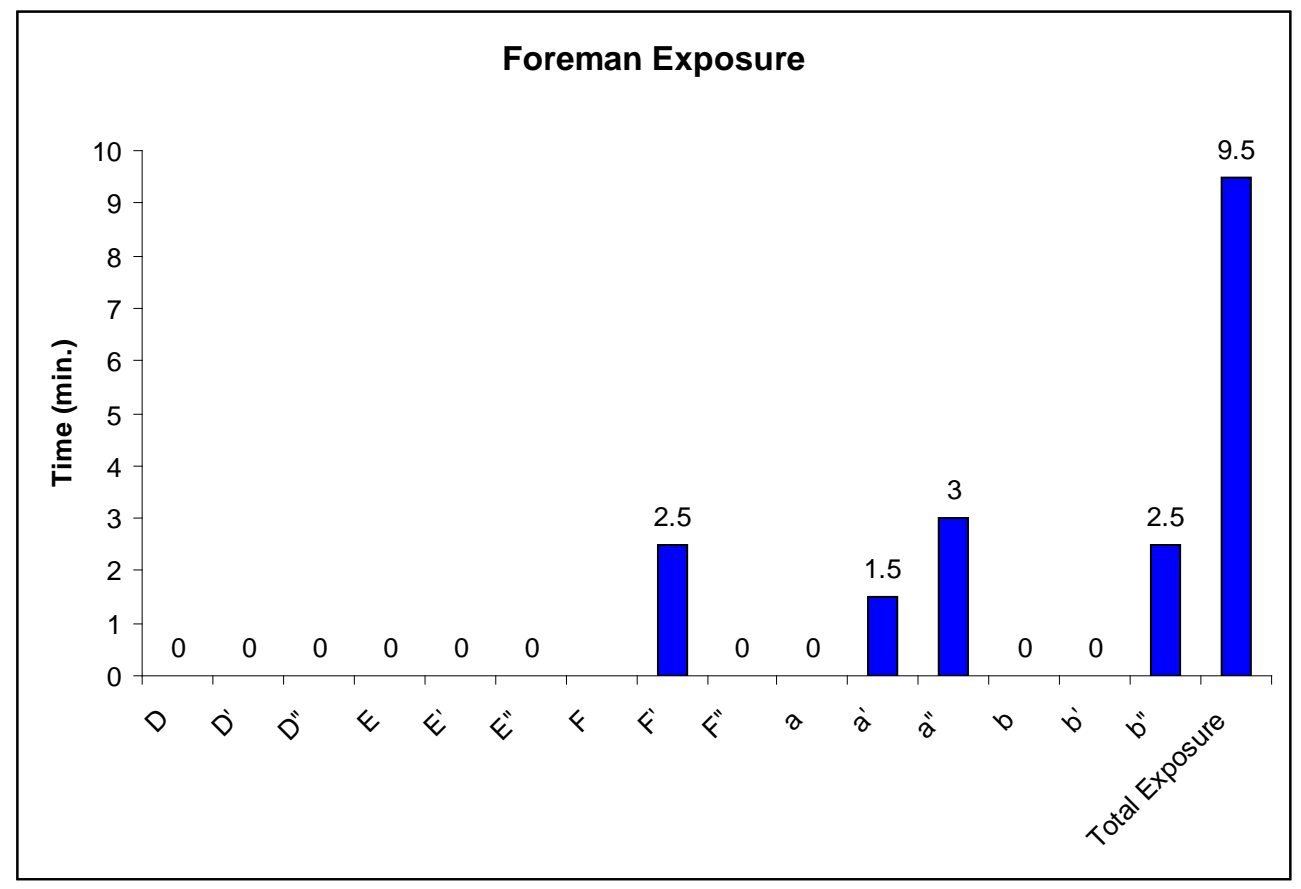

Figure 5.12. Foreman Exposure

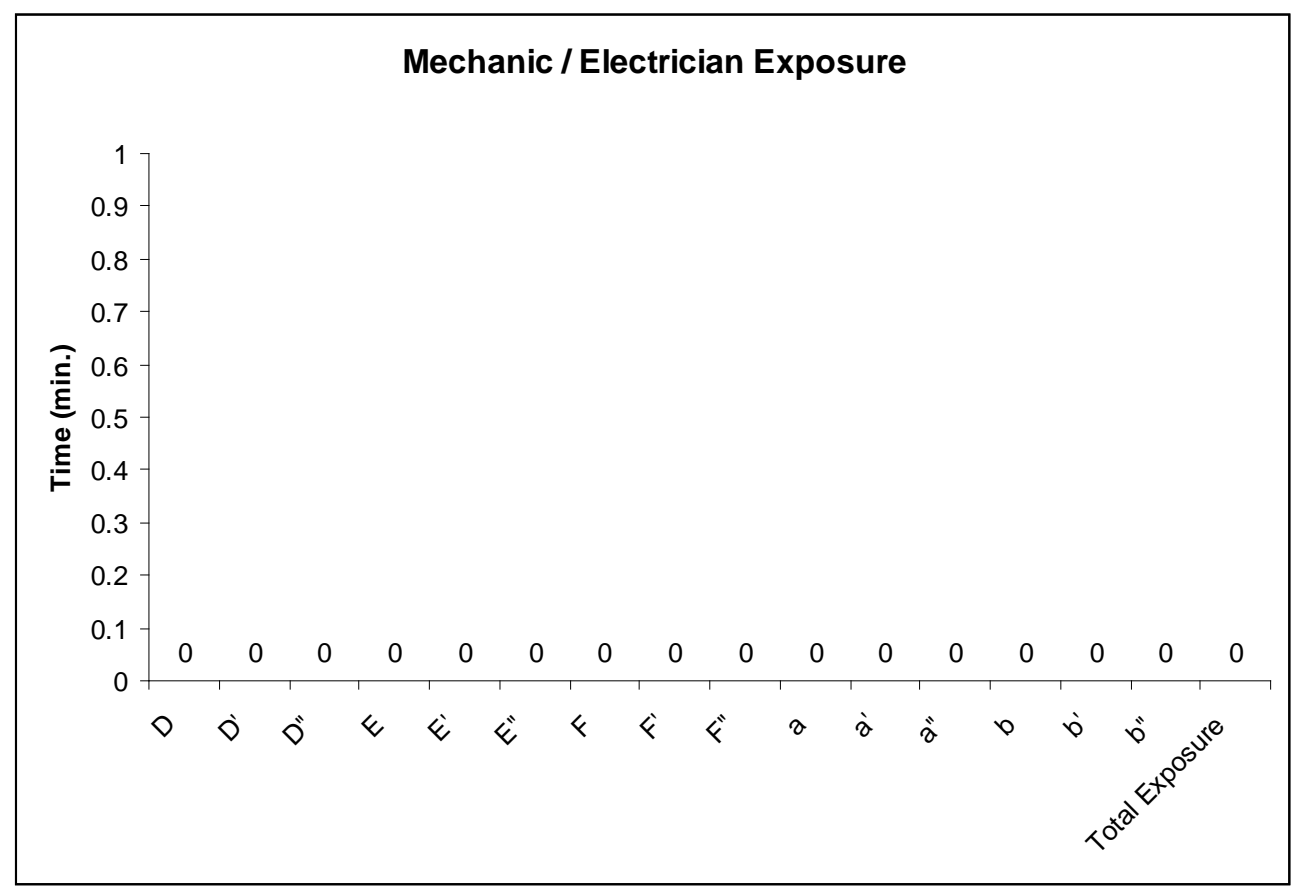

Figure 5.13. Mechanic / Electrician Exposure 
Figure 5.14 represents the exposure time in the active mining zone for members on the section crew (Operators of haulage equipment have been excluded due to the cyclic times in and out of the active mining zone, which made recording very challenging). After a job description's exposure rate is identified, it can be further analyzed to assess training needs. For example, the MRS Operator No.2 was exposed for 31.5 minutes in the active mining zone yet, according to Figure 5.21, only five minutes were spent operating the MRS units during this cut. This highlights a need to further investigate the activity of the MRS operator. This same type of investigation can be conducted for any member of the crew.

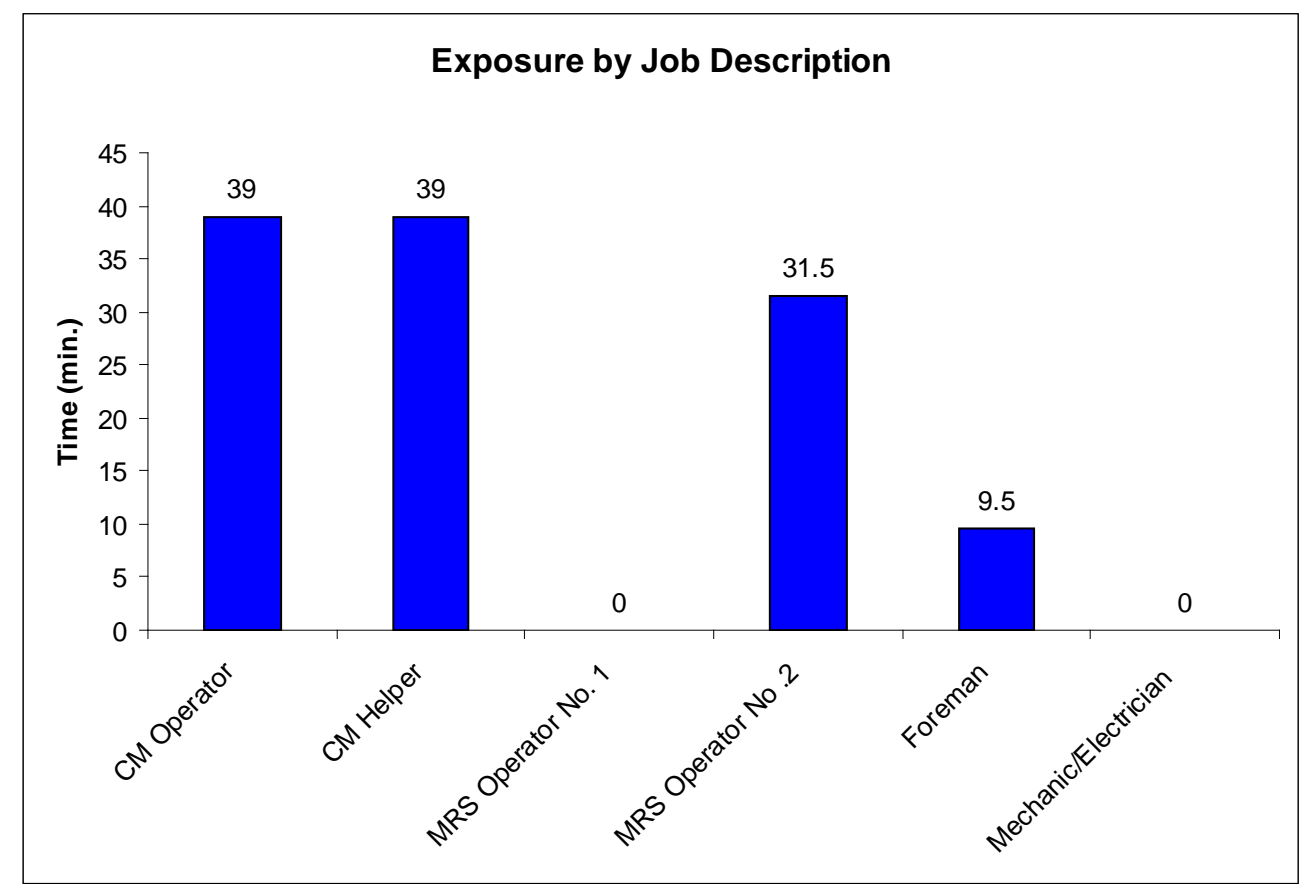

Figure 5.14. Exposure to the Active Mining Zone by Job Description

Activity Timing. As previously stated, once the amount of exposure to the active mining zones has been measured, it is useful to determine what miners are doing during their time in the active mining zones. Figures 15.15 to 15.20 represent the time spent performing various activities by each of the miners that were present in the active mining zone. While most activity descriptions are clear, the difference between the activities "In Between Tasks" and "Observing", requires some clarification. The activity labeled "In Between Tasks" indicates that the miner was not participating in any miningrelated task. The activity label "observing" indicates that the miner was monitoring various aspects of the active mining zone. Information regarding activity timing is useful for determining if hazard exposure times correspond to necessary work being performed. Again, using the MRS Operator as an example, 23.5 minutes were recorded in the active mining zone while the MRS Operator was in-between tasks, indication that he was not performing his specific job or serving any other type of function in place of another crew member. This is a strong indicator, for safety trainers, 
that action can be taken to reduce the MRS Operator's hazard exposure by finding a more suitable location while in-between tasks.

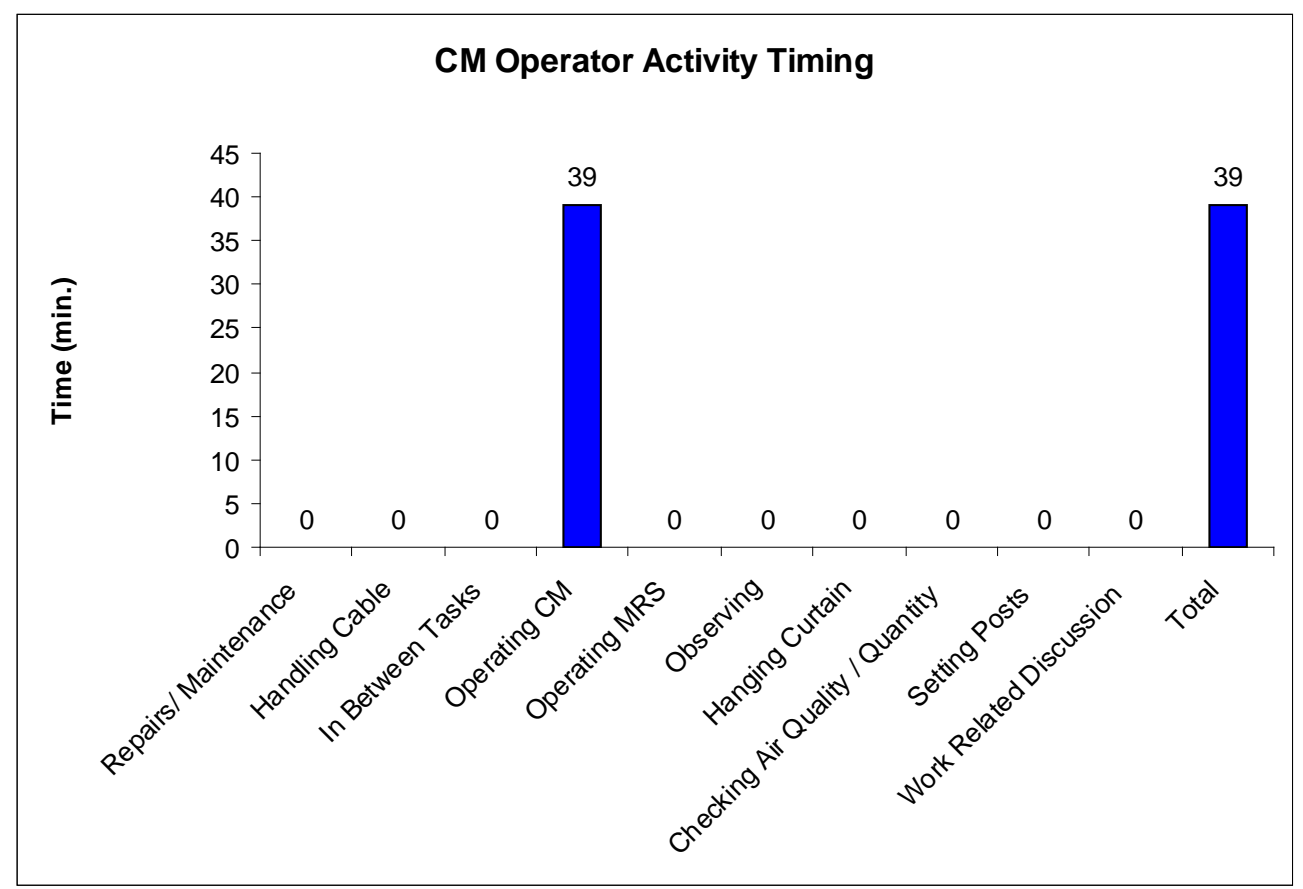

Figure 5.15. Activity Timing for the Continuous-Miner Operator

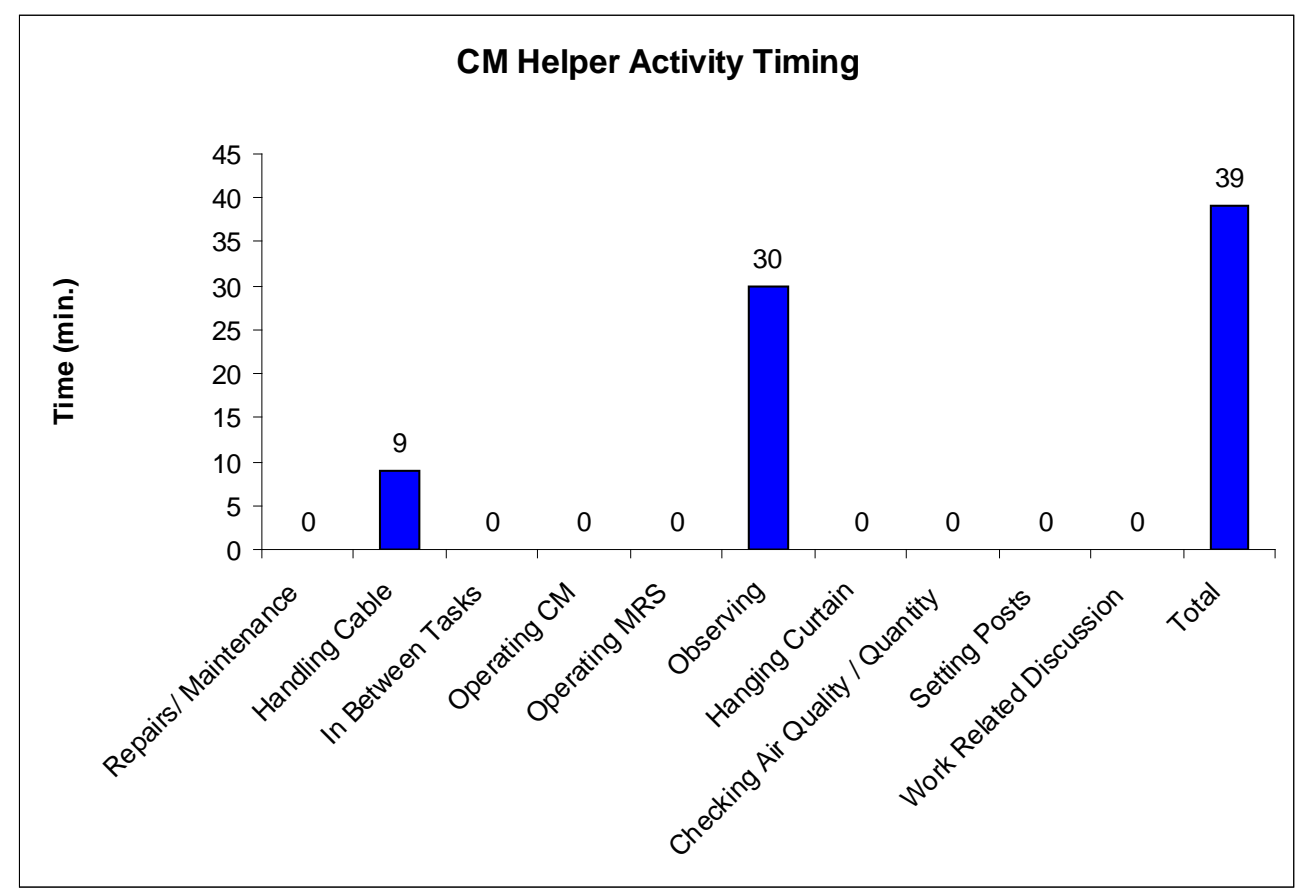

Figure 5.16. Activity Timing for the Continuous-Miner-Operator Helper 


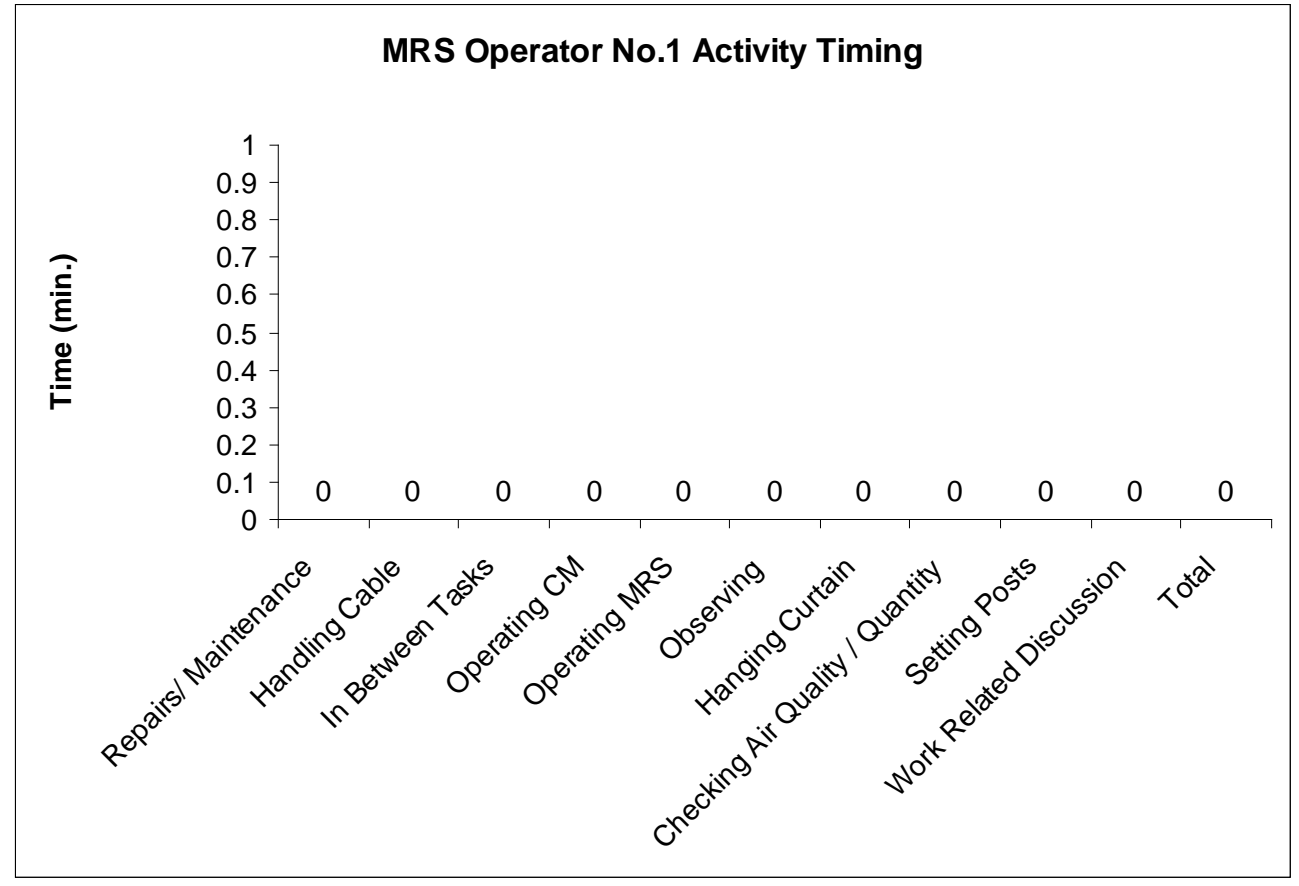

Figure 5.17. Activity Timing for the MRS Operator No. 1

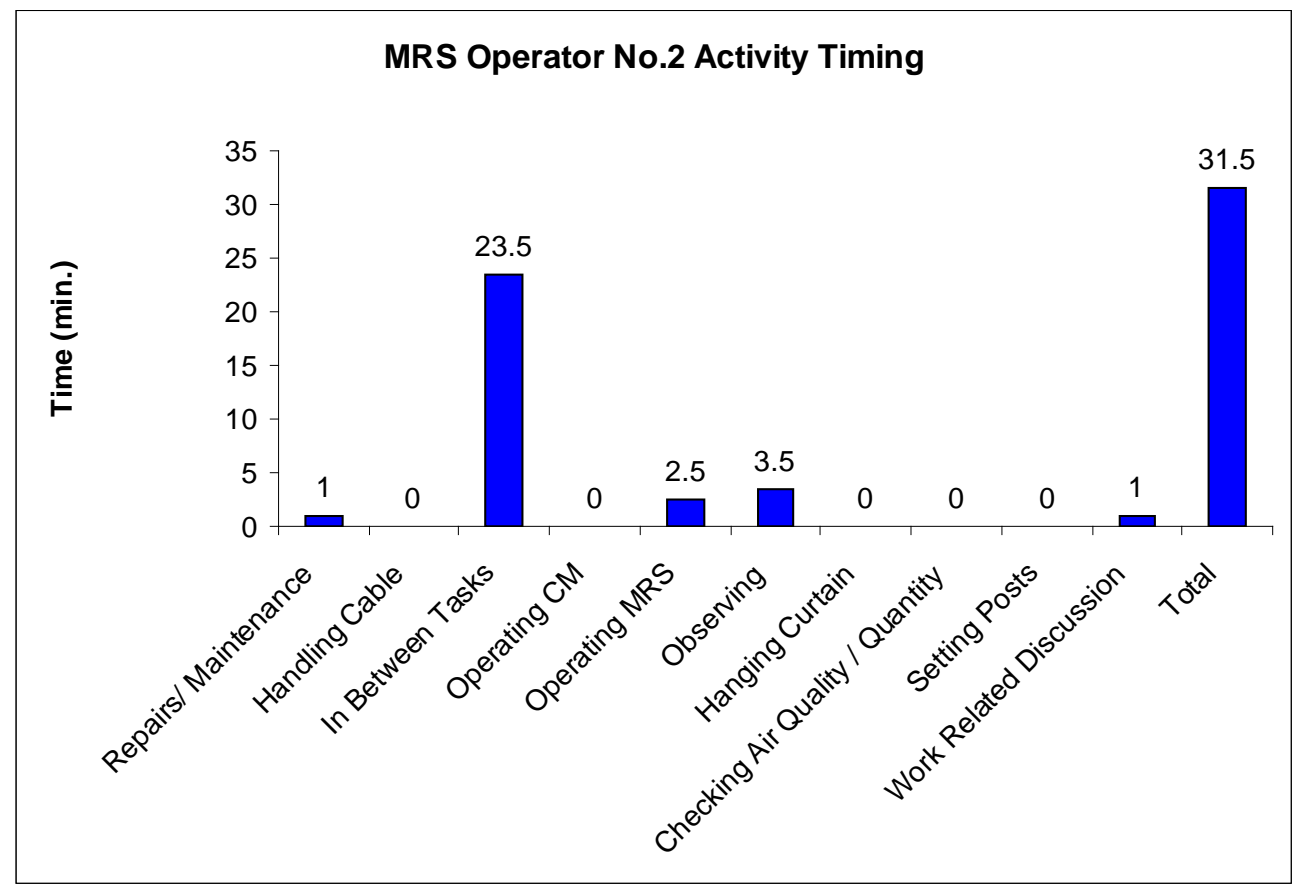

Figure 5.18. Activity Timing for the MRS Operator No. 2 


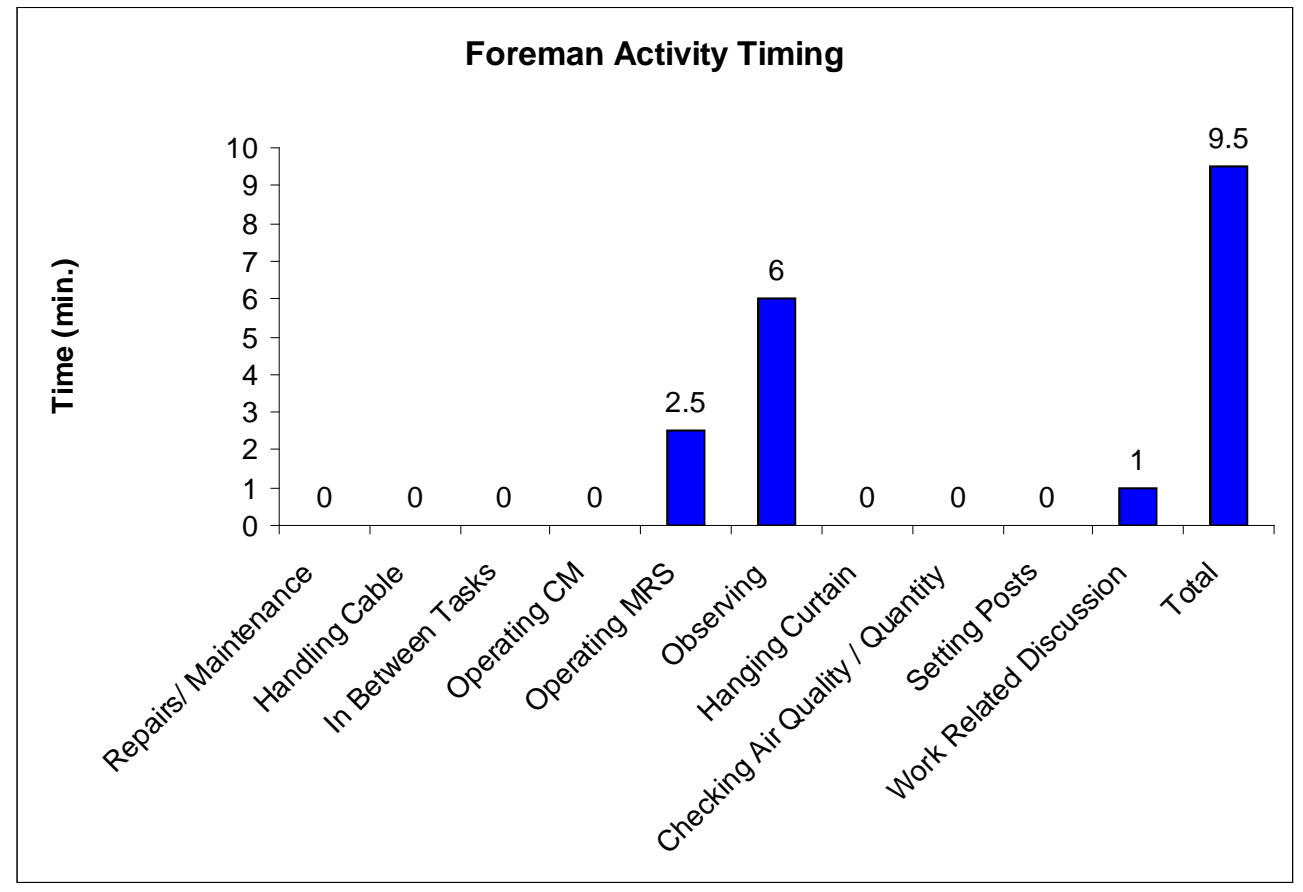

Figure 5.19. Activity Timing for the Foreman

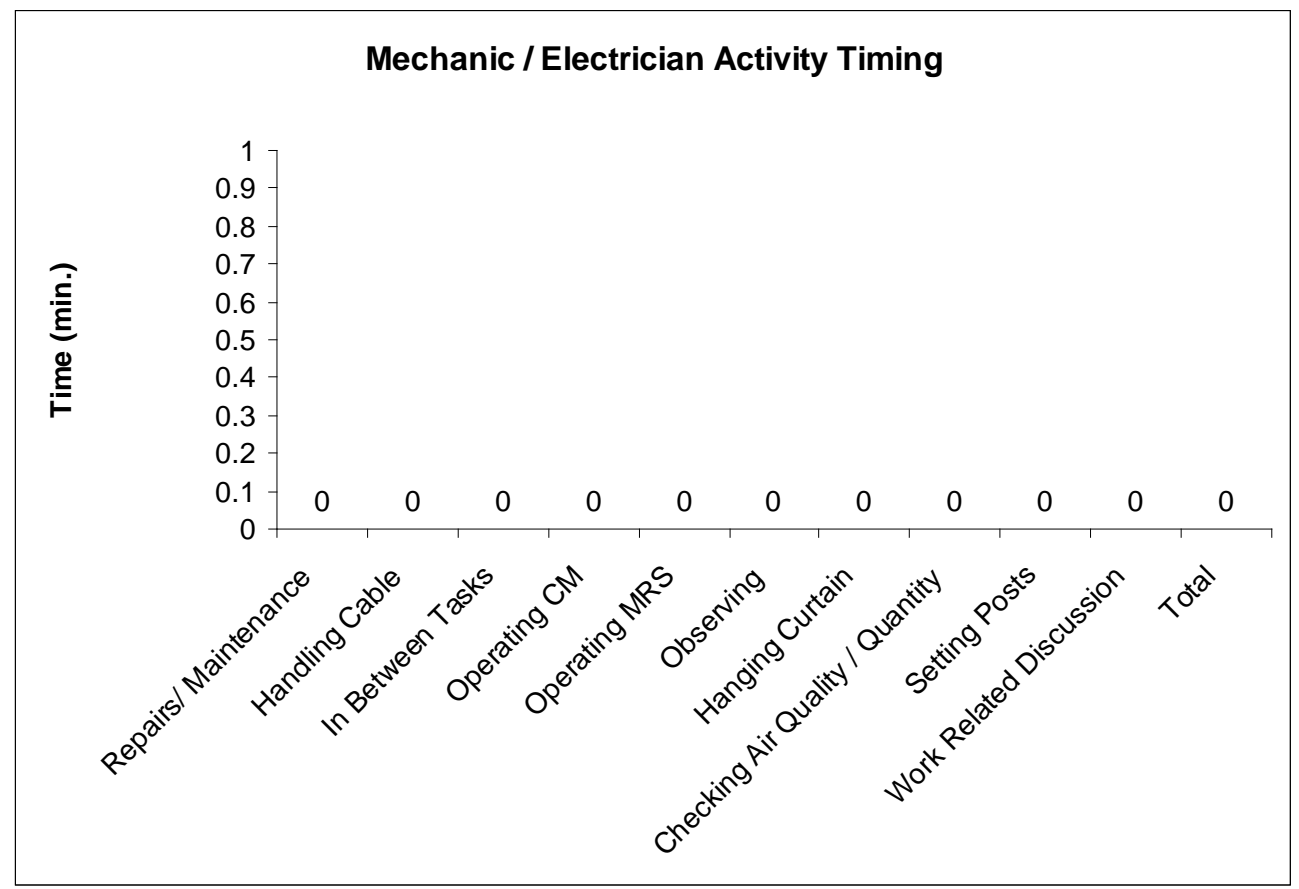

Figure 5.20. Activity Timing for the Mechanic / Electrician

This type of representation of the information gathered during the Activity and Location Monitoring Observation can serve dual roles, both highlighting needs for safety training as well as providing information that can lead to process optimization. 


\section{Cut No. 3}

Figure 5.21 shows the location of the cut being analyzed, as well as prior and future cuts and the location of the MRS units. The selected observation point again was located in zone b". The total time to extract the cut and reposition the MRS units was approximately thirty minutes. During this time, the location and activity of everyone entering the active mining zone, with the exception of haulage operators, was recorded on the Activity and Location Monitoring form, similar to previous cuts.

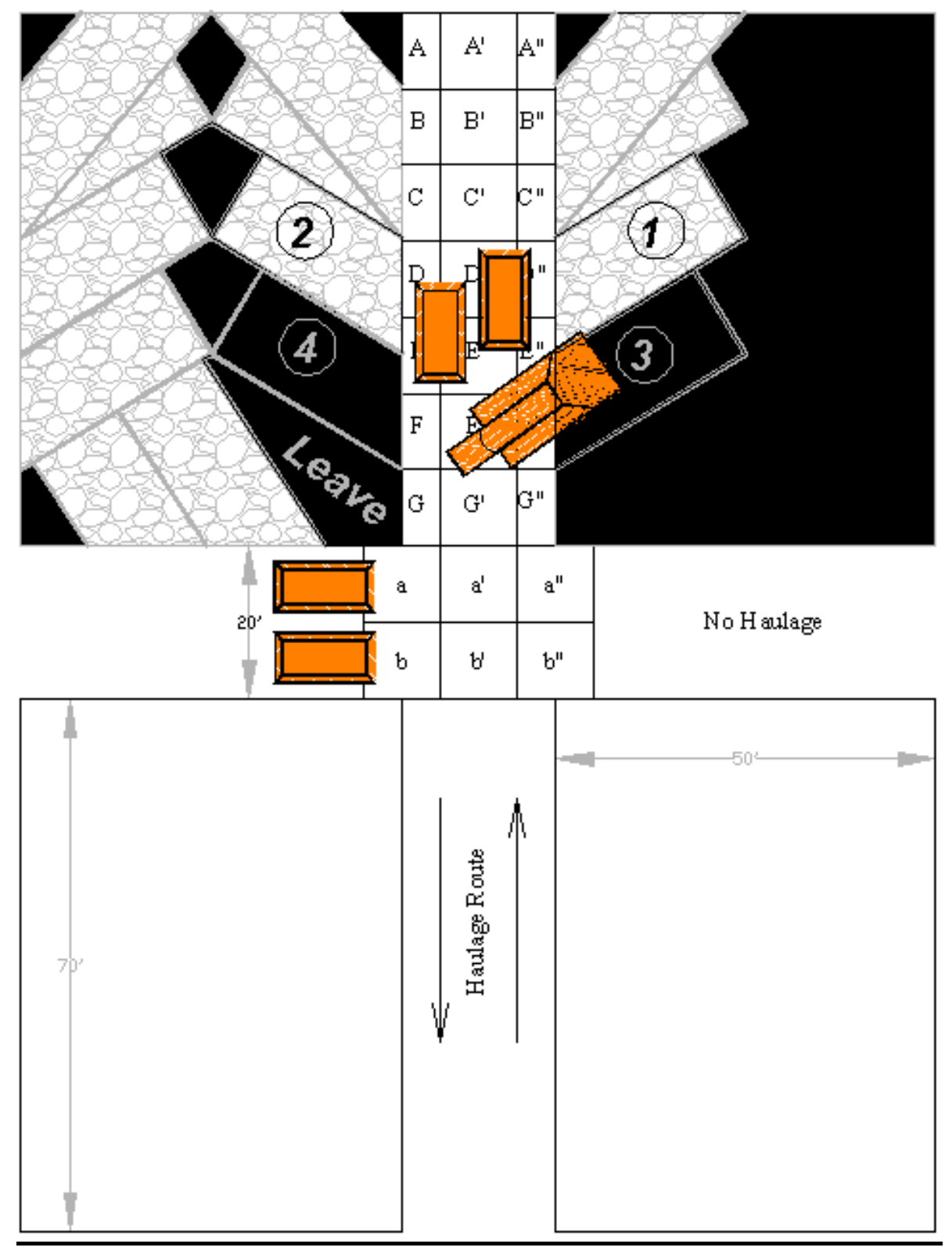

Figure 5.21. Area Division and Cut Numbering for Cut No. 3 
Exposure to the Active Mining Zone. As demonstrated previously in the Cut No. 2 analysis, graphs representing exposure times by zone have been created (Figure 5.22 to 5.28). For this cut, there were still significant levels of exposure by the continuous miner operator and helper as would be expected due to their job descriptions. There was also a level of exposure time by MRS operators that does not correspond to their typical job activities, as also witnessed in the previous cut. However, for this cut, the foreman remained in the active mining zone for the entire cutting process, as well as the MRS relocation, and as a result, the Foreman had the highest exposure time for this cut.

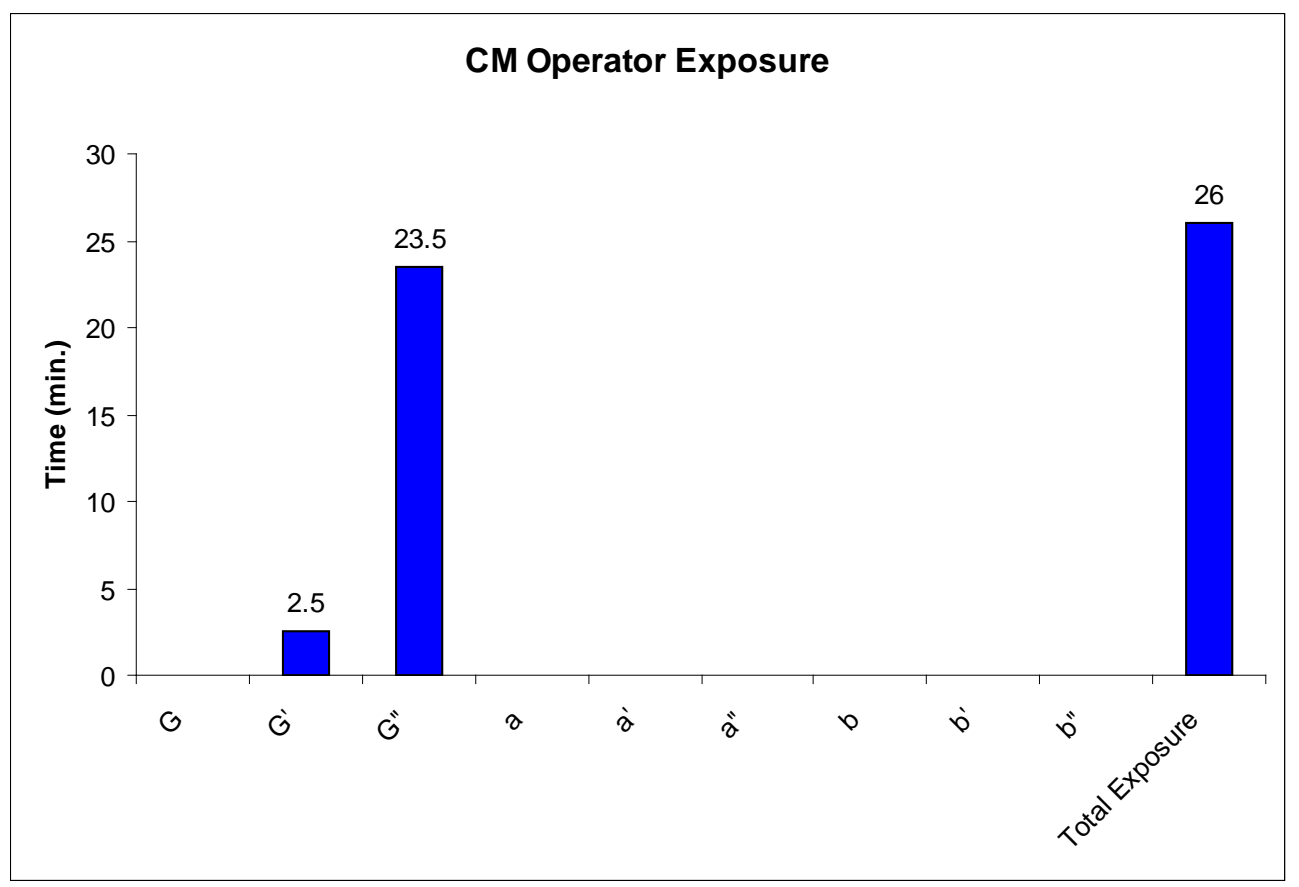

Figure 5.22. Continuous-Miner Operator Exposure 


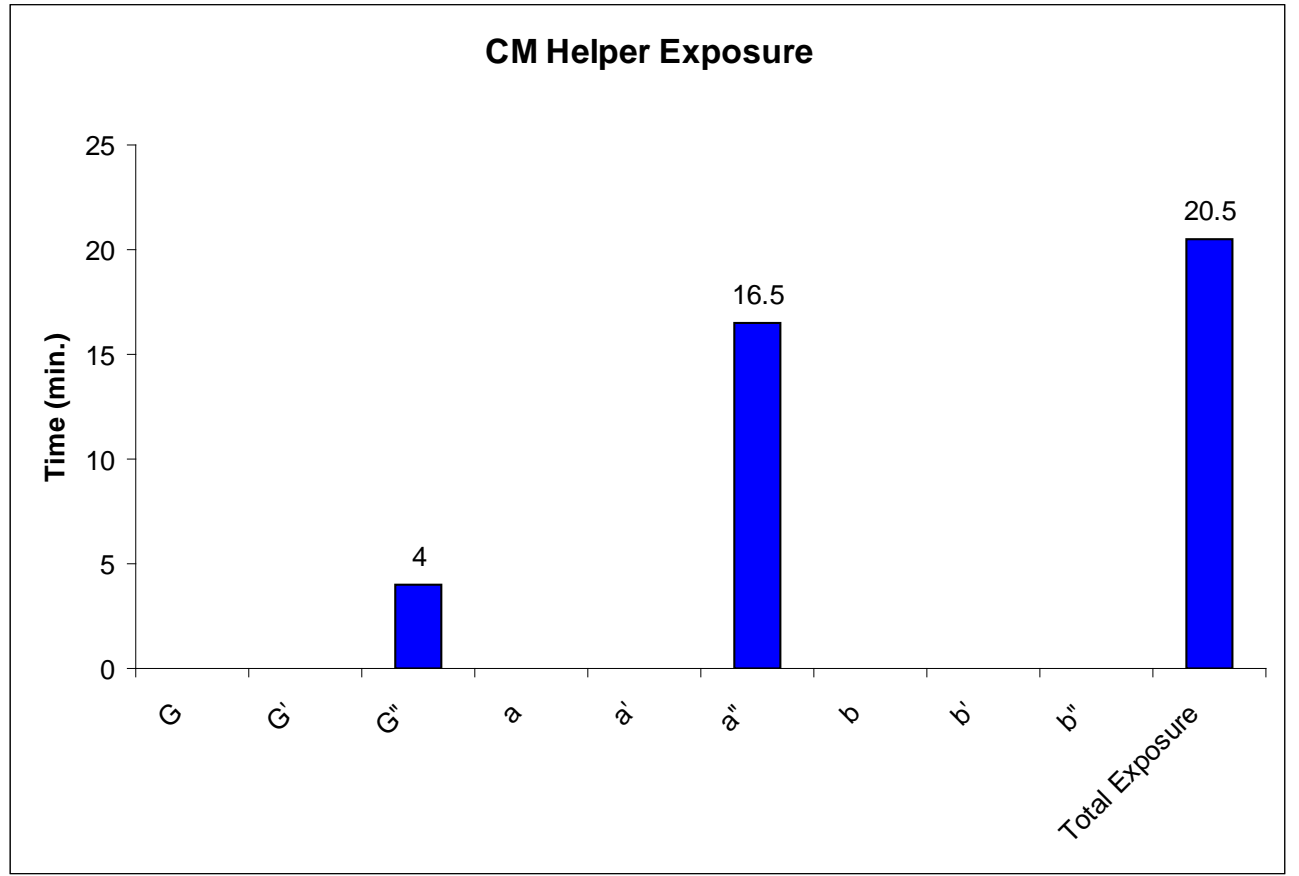

Figure 5.23. Continuous-Miner-Operator Helper Exposure

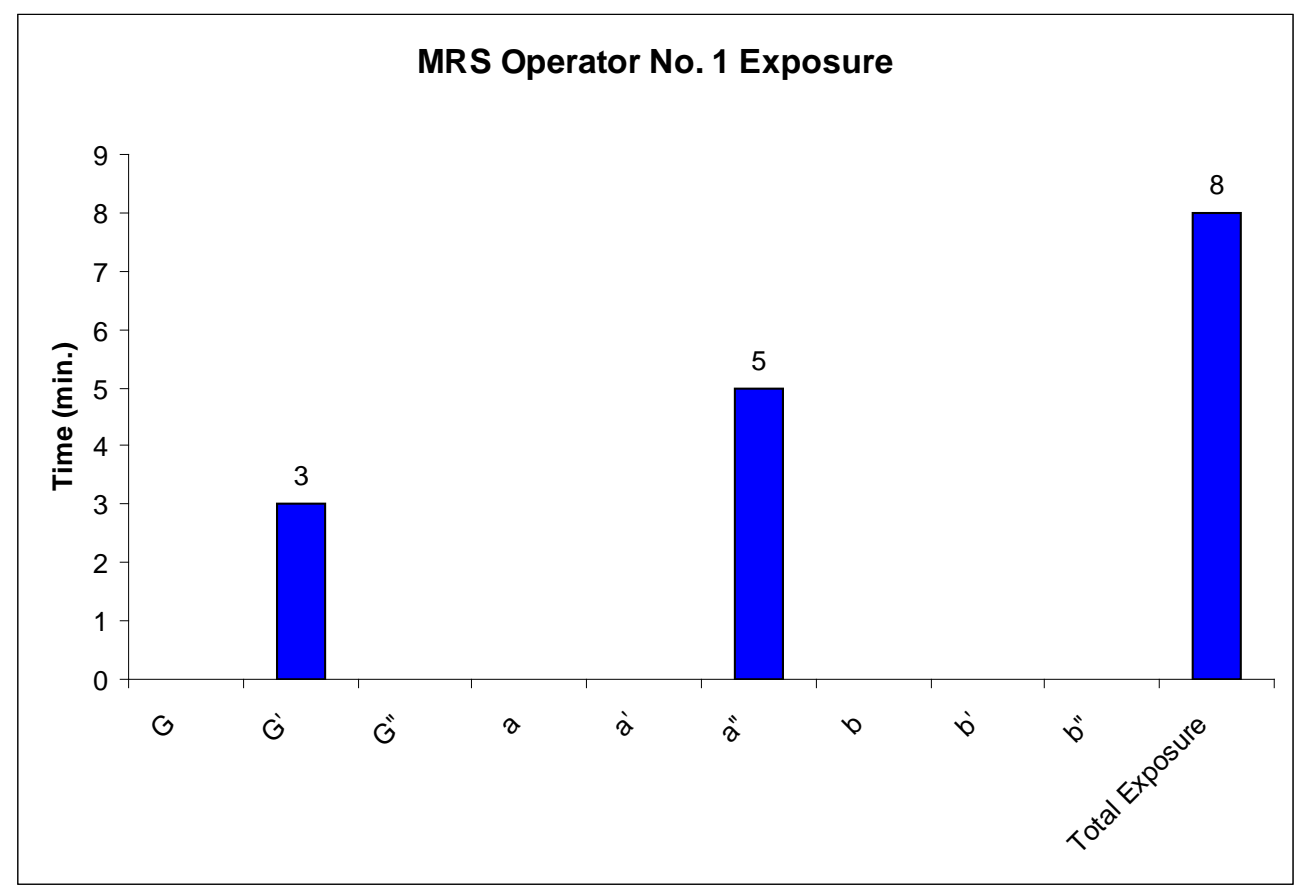

Figure 5.24. MRS Operator No. 1 Exposure 


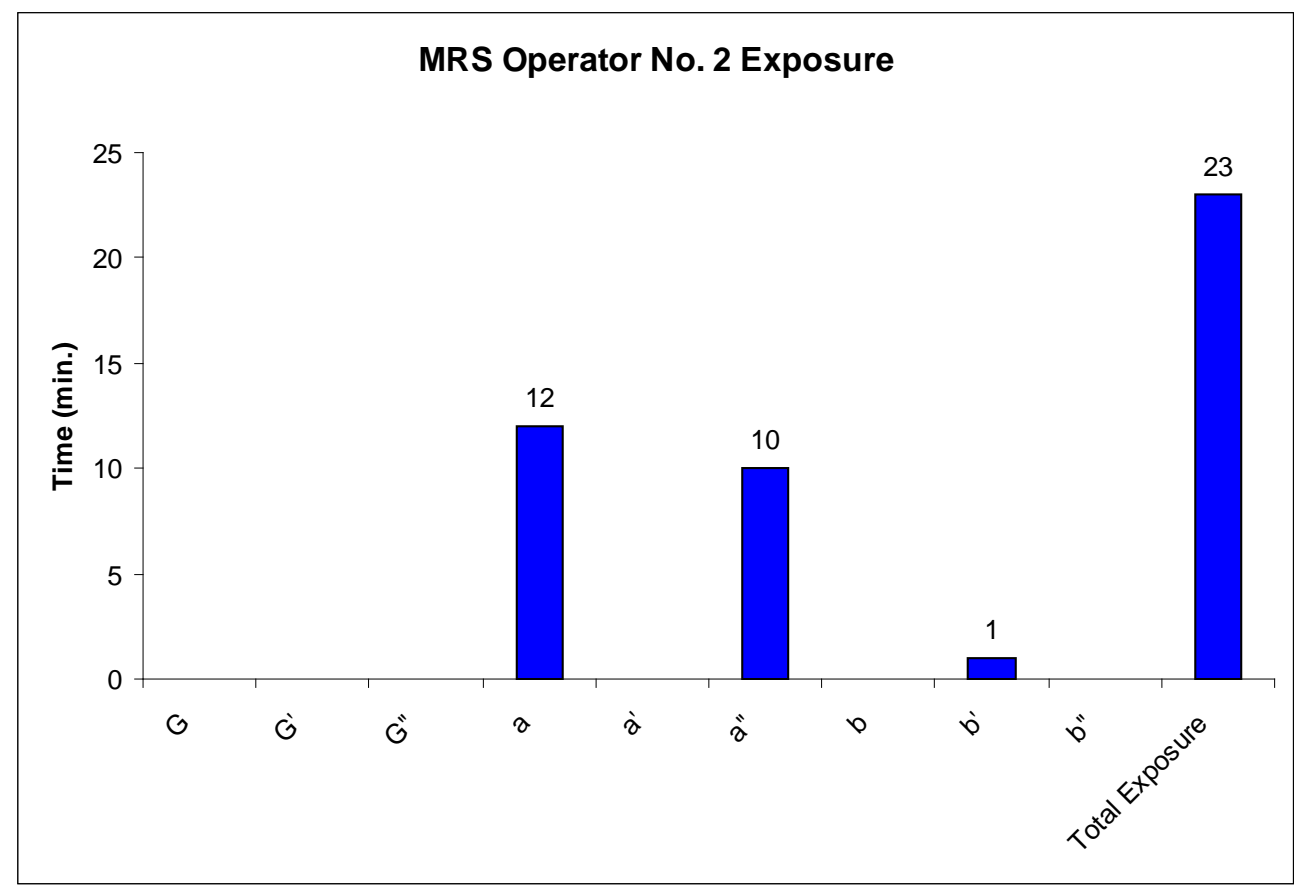

Figure 5.25. MRS Operator No. 2 Exposure

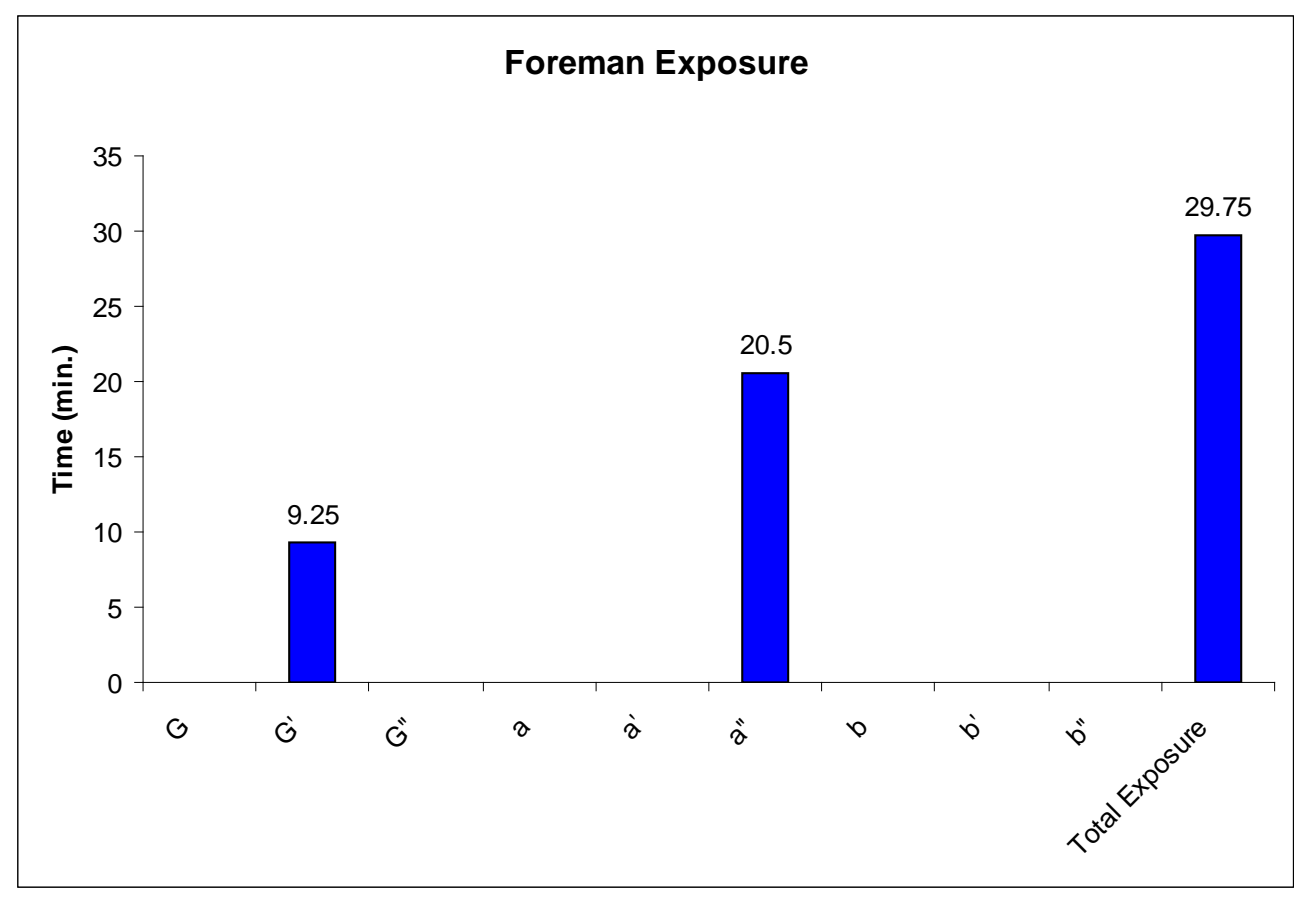

Figure 5.26. Foreman Exposure 


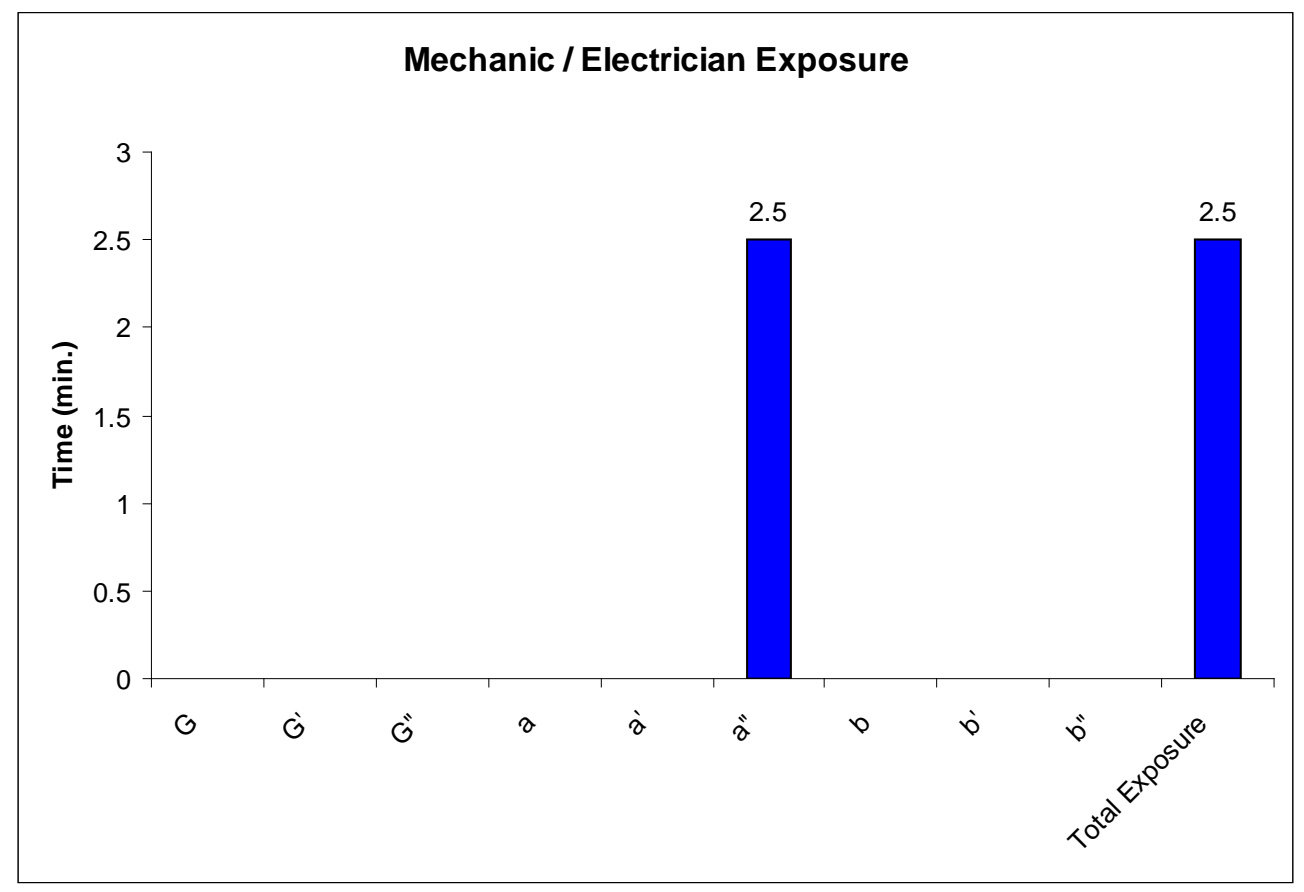

Figure 5.27. Mechanic / Electrician Exposure

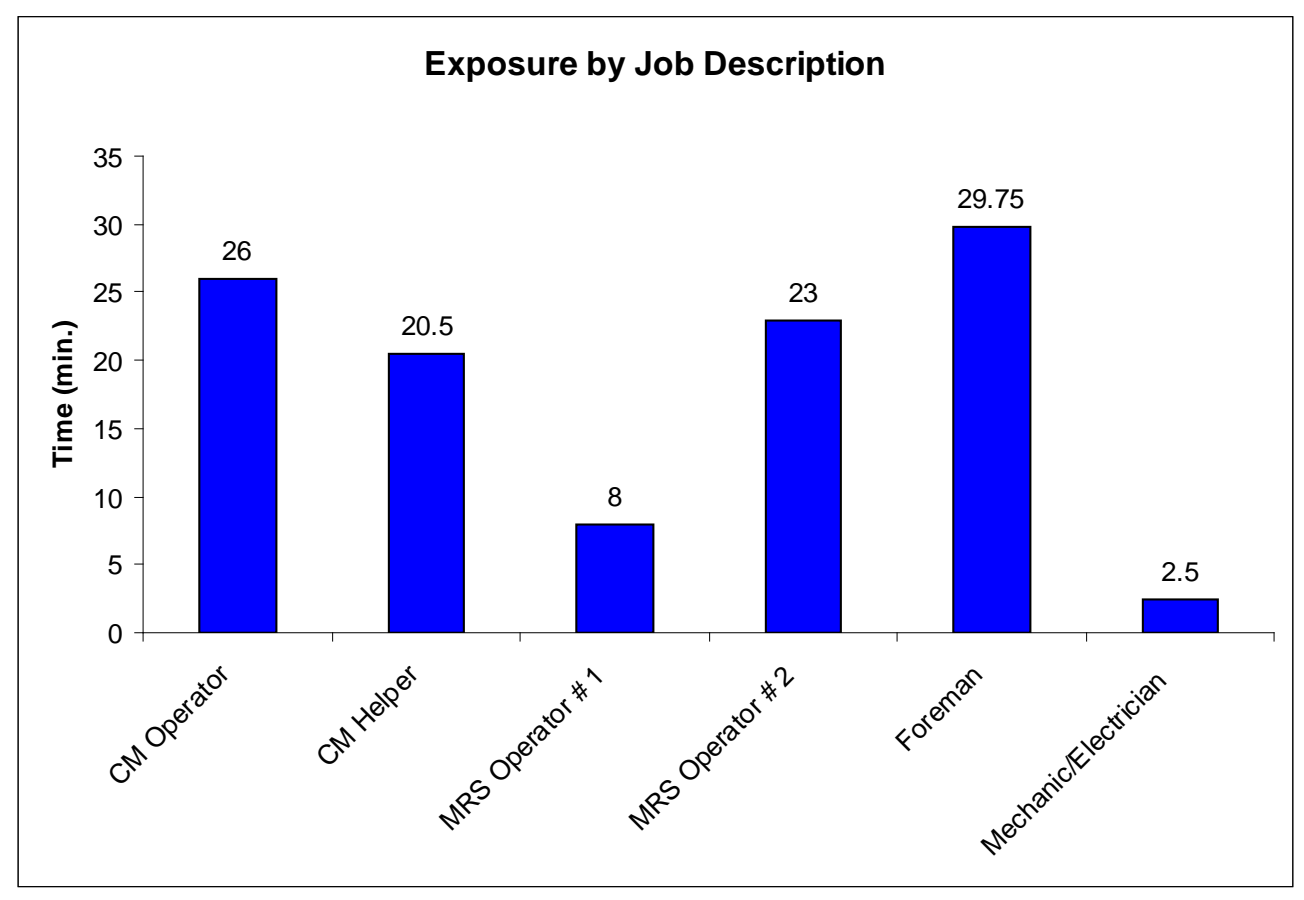

Figure 5.28. Exposure to Active Mining Zone by Job Description 
Activity Timing. Figures 5.29 to 5.34 present the amount of time each miner spent performing an activity. Consistent with Cut No. 2, the MRS Operator No. 2 had the most time "In Between Tasks" in the active mining zone for Cut No. 3. Even though the MRS Operator No. 1 was present in the active mining zone, the operator was actively engaged in a work activity during that time period.

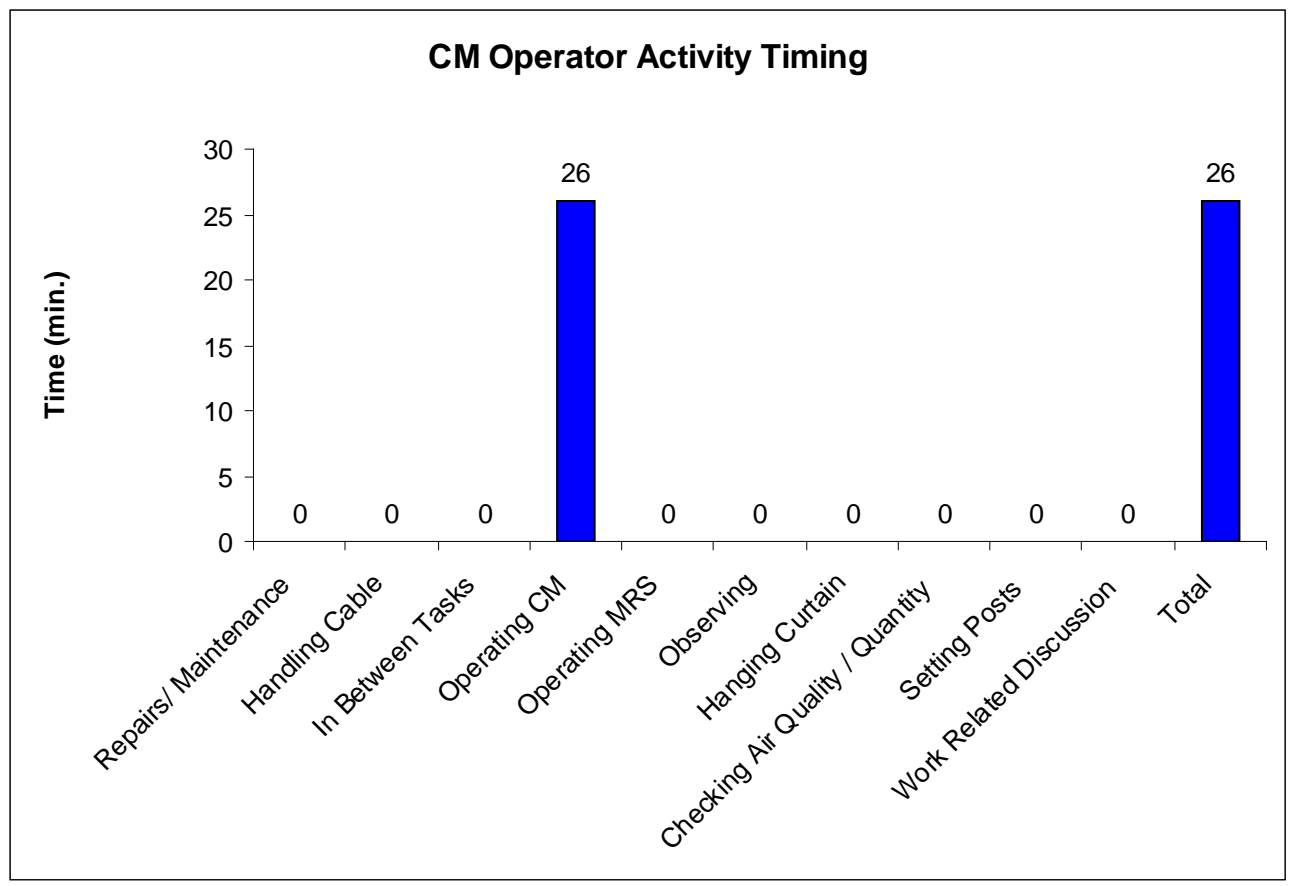

Figure 5.29. Activity Timing for the Continuous-Miner Operator

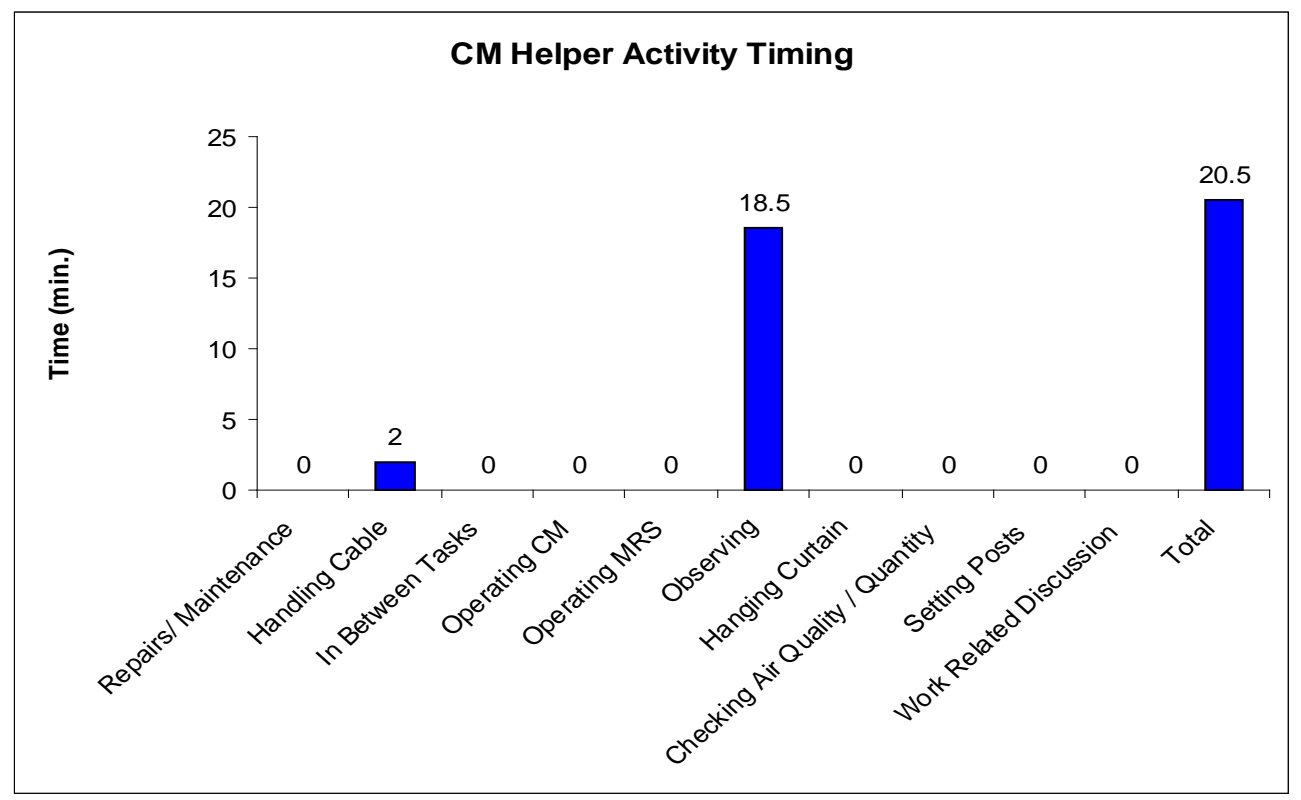

Figure 5.30. Activity Timing for the Continuous-Miner-Operator Helper 


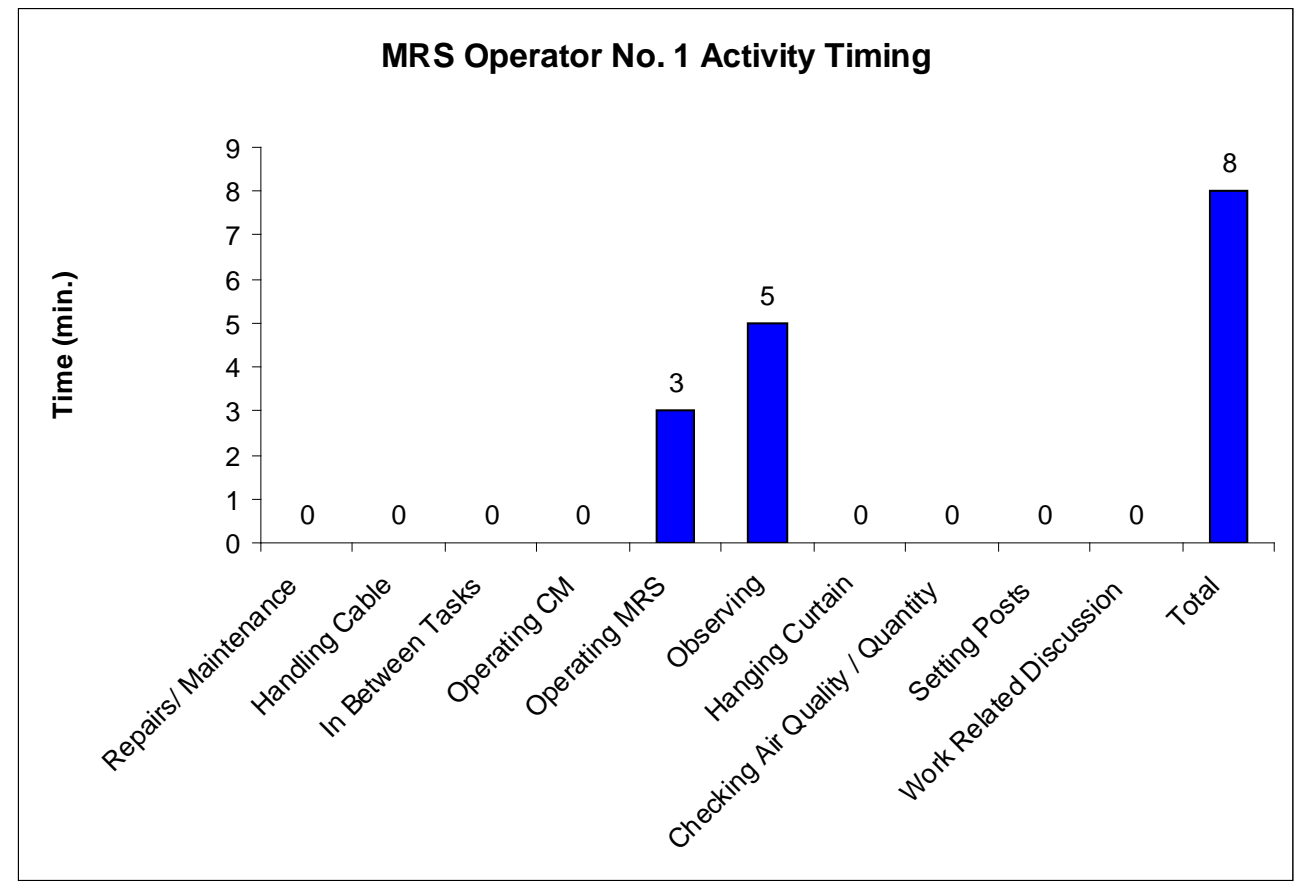

Figure 5.31. Activity Timing for the MRS Operator No. 1

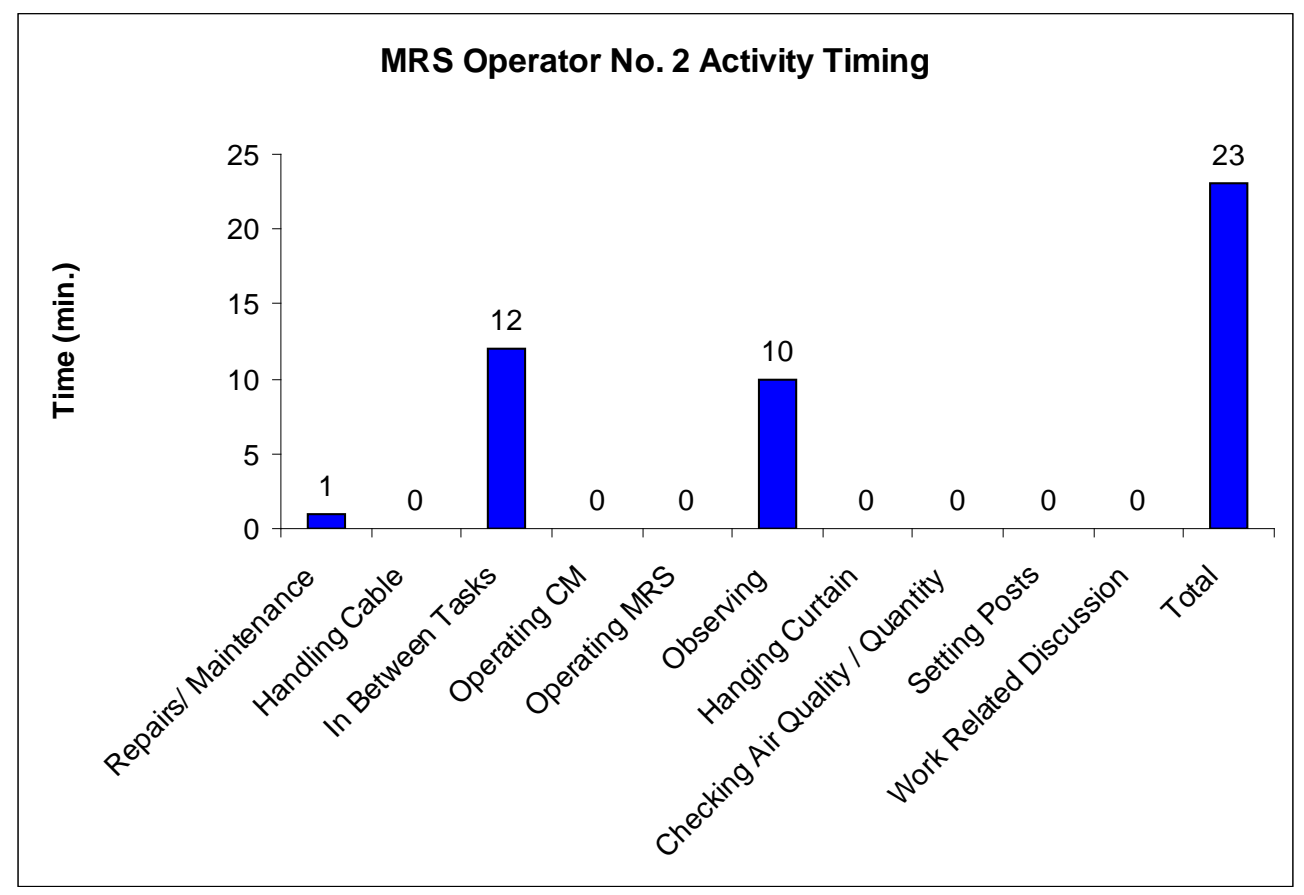

Figure 5.32. Activity Timing for the MRS Operator No. 2 


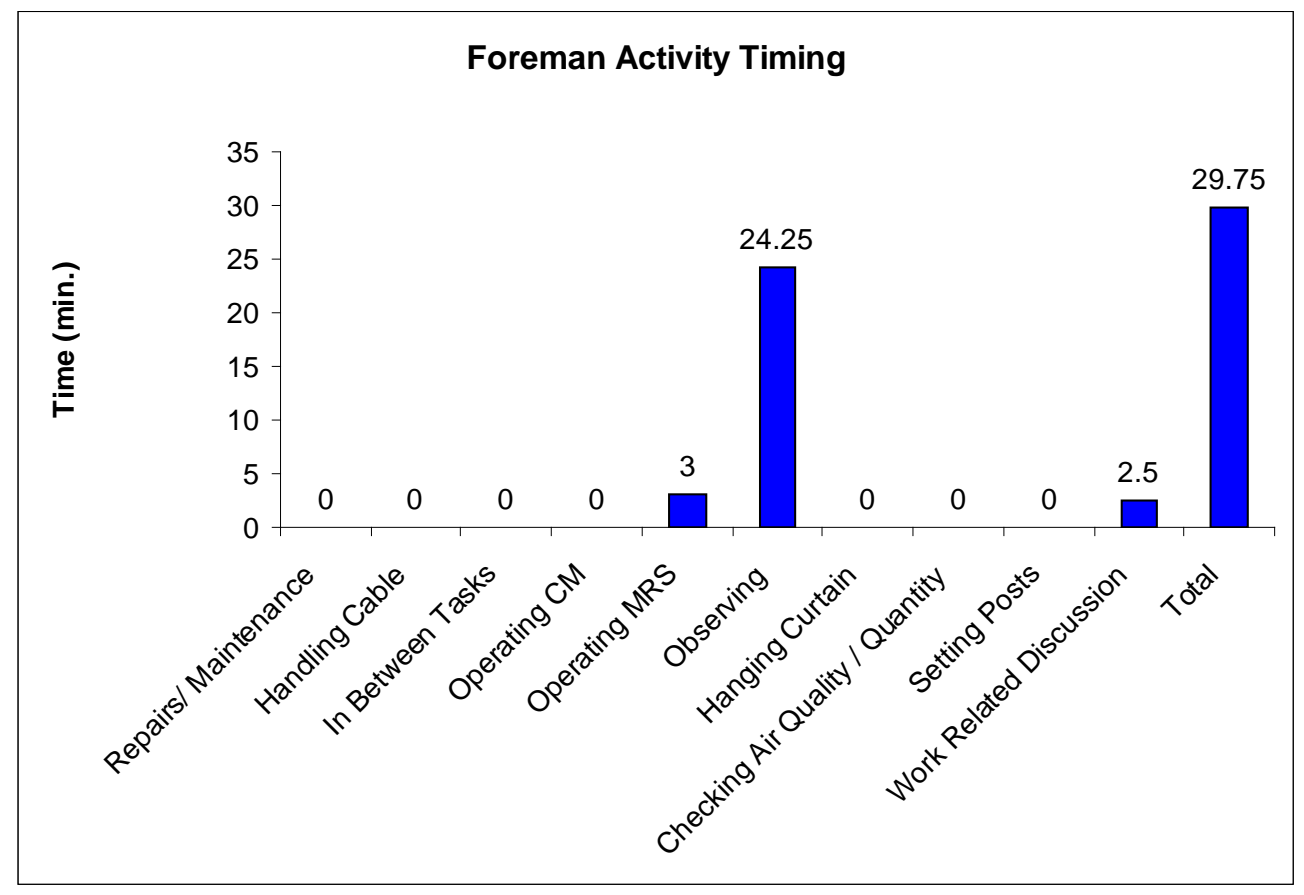

Figure 5.33. Activity Timing for the Foreman

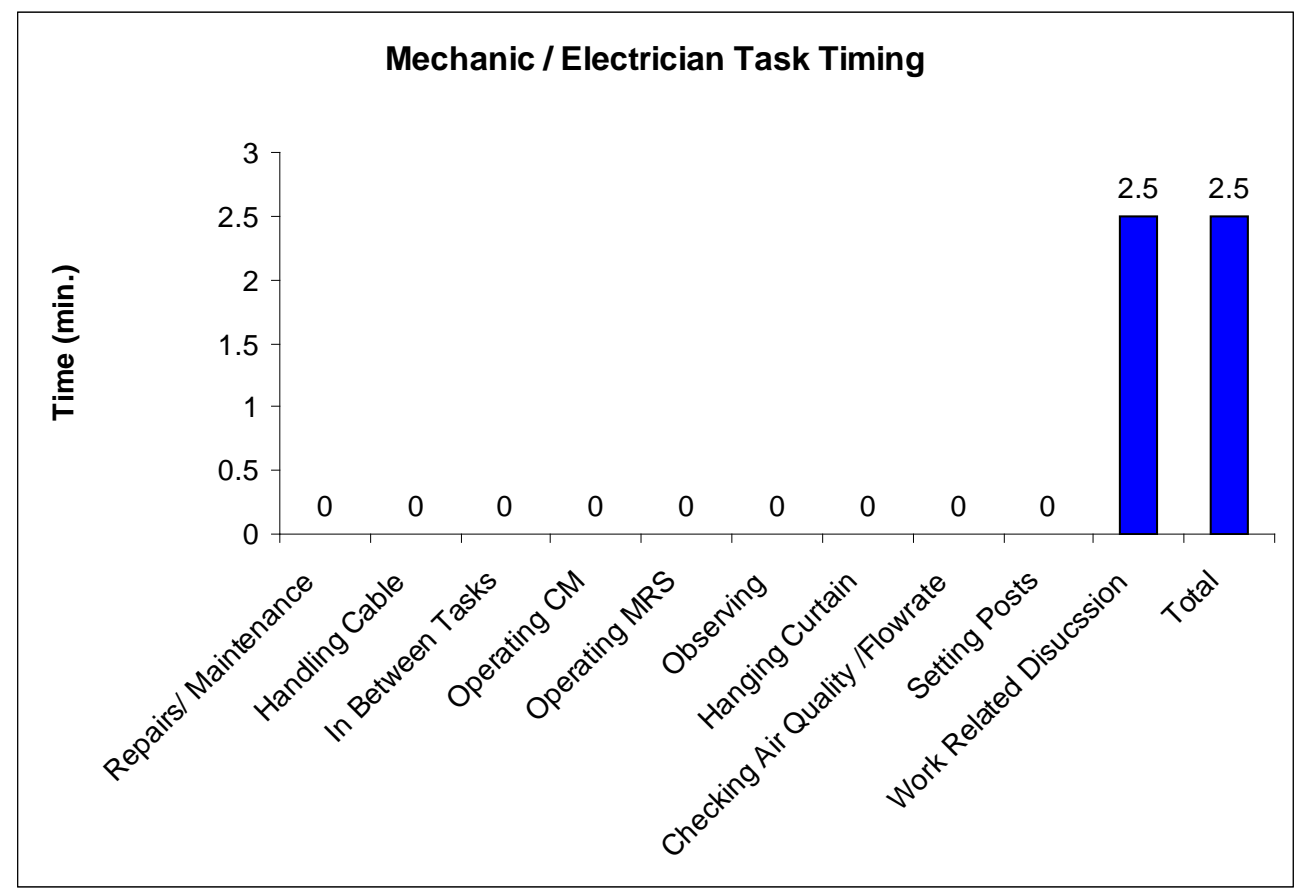

Figure 5.34. Activity Timing for the Mechanic / Electrician

\section{Summary of the Results}

The results of this sampling strategy make it possible to quickly identify training needs. The results of the Unsafe Performance Observation show the need to focus 
training efforts on safe positioning of the body to minimize exposure to machine and geological hazards, as well as proper operator positioning for anyone on the section that might be called upon to operate equipment at the face. These results are supported by the Activity and Location Monitoring results, which show improper body placement during the mining process by both equipment operators and other personnel. The amount of hazard exposure to personnel that were not essential to the cutting and loading process, as well as the high levels of time "In Between Tasks" observed in the active mining zone, are both strong indicators of unnecessary lingering in the active mining zone. With this information, mine-safety trainers can be equipped to more effectively target problem areas such as these. Doing so could dramatically improve the overall safety and performance of the pillaring operation. The strategy proved to be very applicable, with no major difficulties in its utilization encountered during this field application.

\section{Mine No. 2 Site Description and Summary}

Mine No.2 is located in Central Appalachia. At the time of the field application, the mining height varied between 4.5 and 6 feet. The immediate roof consists of 0 to 2 feet of shale, (depending on location within the mine), below an approximately $10 \mathrm{ft}$ thick layer of sandstone. The bolting pattern utilizes a 4-ft-by-4-ft pattern primarily employing 48-inch roof bolts. The section contains a Joy Model 14CM continuous miner, a Stamler continuous-haulage system, a Long- Airdox scoop, and two 800-ton Fletcher mobile roof supports The mine operates three shifts per day (two production, one maintenance), seven days per week. Each operating section is staffed by a crew of 12-13 miners; however, only 7 to 8 miners consistently work in the active mining zone. Pillar dimensions encountered during the field application were 60 feet wide and 60 feet long. Each entry and crosscut is 20 feet wide (Figure 5.35).

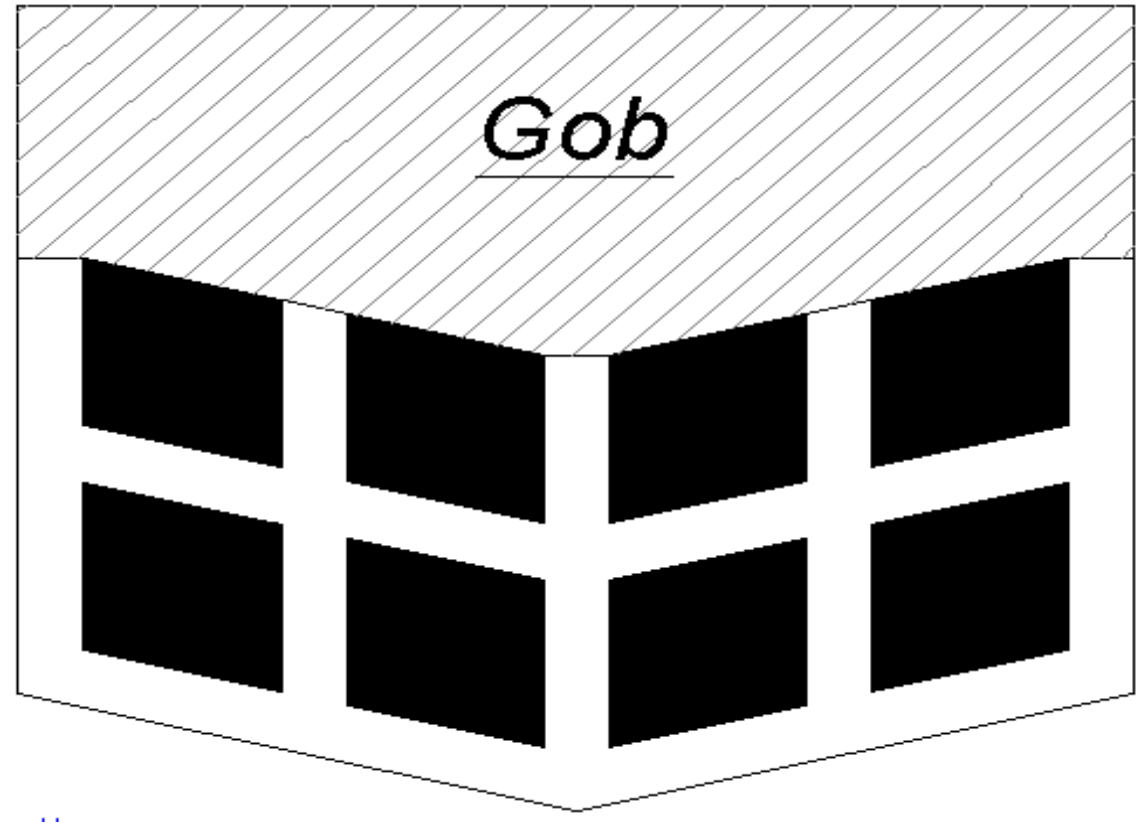

Figure 5.35. Mine No.2 Section Layout 
Annual production is estimated to be approximately one million clean tons. During the observation, pillars were being extracted from under approximately 1650 feet of cover. The age of the pillars being mined during the observation was approximately six years. The coal ribs showed little sign of spalling and were in very good condition. The field application of the performance sampling strategy took place over three days, the initial day was spent getting acquainted with the mine and the mining methods utilized. The following two days were spent underground with the dayshift crew for ten hours per day.

During the course of this field application, the sampling strategy, again, proved to be applicable and effective. Management was familiar with the hazards and risk factors at their mine, and the Pre-Mining Risk Matrix was successfully completed. Unsafe performances, such as horseplay, failure to utilize personal protective equipment, as well as unnecessary exposure to machinery and geological hazards, were frequently identified using the Unsafe Performance Observation Form. The Activity and Location Monitoring Form was successfully utilized to monitor mineworkers in the active mining zone. However, there were conditions encountered during this field study that posed some difficulties for the sampling strategy. The first difficulty arose from the presence of the continuous-haulage equipment. The equipment utilized on a continuous-haulage section is very large and reduces the mobility of the observer. The combination of continuous haulage equipment and the layout of the angled pillars restrict the observer's capability by both obstructing vision, as well as reducing the number of safe vantage points due to an increased number of machinery pinch points. However, as mining progresses on a continuous haulage section, safe vantage points for observation do become available. Knowledge of the location of the continuous-haulage machinery as it moves from pillar to pillar is required to accurately identify these safe vantage points. The routing of the continuous-haulage equipment varies from mine to mine, making it impossible to designate safe vantage points that will work in every mine. Therefore, in order to execute the sampling strategy on a continuous haulage section, it is important for an observer to carefully plan for locations on the section, as well as times when safe vantage points are available. By remaining in the active mining zone without a safe vantage point, the observer is then contributing to the risk to be minimized. The sampling strategy can prove equally effective on continuous-haulage sections but, in order to be successful, more careful planning and timing is required.

The second difficulty encountered involved the attitude of the section personnel in regard to their involvement with the sampling strategy. Based on experience gained during the first field application, it was anticipated that both upper-level and line management would welcome observers that are conducting safety and performancerelated observation. This proved to also be true during the second field application. The difficulty was encountered with the mineworkers. In the previous application, mineworkers were initially hesitant about being monitored but, after some brief explanation and reassurance that no punitive measures could be taken based on the results of the observation information, most were very informative and cooperative. The same level of cooperation from the mineworkers was not encountered during the second visit. The lack of trust in the observer and disregard for the observation that was being performed made the field application difficult. It became evident that, in order for an observation strategy such as the one designed in this research to be effectively 
utilized, the observer would need to have the full support of upper-level management, in the form of authority to direct the mineworkers or the accompaniment of a manager with such authority. Despite the challenges encountered, the sampling strategy proved applicable. In future field applications of this strategy, the difficulties encountered during this observation could easily be overcome by better planning of the observation times, as well as utilizing an observer that can exert influence on the mineworker, required.

\section{Estimation of Time Requirements}

The time required for one observer to complete the sampling strategy, and process the information is approximately one week, assuming eight to ten work hours per day. During the field applications, the observer dedicated the first day to becoming familiar with the mine and mineworkers, as well as completing the Pre-Mining Risk Matrix. The second day of the field application was dedicated to the Unsafe Performance Observation. The third day was dedicated to the completion of the Activity and Location Monitoring Form. Upon completion of the sampling strategy in the previously described sequence, approximately two days are required to process the information gathered during the sampling strategy, and present it in a format suitable for training purposes.

\section{Chapter Summary}

Throughout the course of this research, multiple risk factors that contribute to pillaring risk have been identified. These factors were categorized as geotechnical or personnel performance related. Focus was then given to the personnel-performance related risk factors having a significant impact on pillaring risk that were observed during mine visits, or noted while reviewing fatality reports. During the development of the performance-based sampling strategy, the goal was to develop a tool for use by safety managers that could identify these high-priority risk factors, such as unnecessary congregation or lingering in the active mining zone, improper location while performing mining-related tasks, unnecessary exposure to machinery and geological hazards, improper operation of equipment and horseplay, as well as many others.

During the field applications, the sampling strategy proved to be very successful in exposing these basic causes of accidents and injuries. Using the Unsafe Performance Observation Form, the observer is able to document the occurrence and frequency of many high-priority risk factors such as improper operator positioning, horseplay, unnecessary exposure to both geological and machinery hazards and lingering in the active mining zone. The completed Activity and Location Monitoring Forms and their corresponding graphical representation provided clear quantitative results regarding unnecessary lingering or unsafe positioning, thus enabling safety managers to pinpoint the location and duration of unsafe positioning and identify those mineworkers that are most at risk.

Although not all at-risk performances were observed, the successful observation of the performances discussed in this section indicates that similar success can be 
achieved in documenting all other unsafe performances that the strategy was designed to target.

During the course of both field applications, the sampling strategy proved to be applicable to the varying mining conditions. The forms were appropriately designed and required only minor modification from mine to mine, and the flexibility of the forms made these modifications possible. The components of the sampling strategy complemented each other and provided clear and well-documented evidence of performance issues that span from management to mineworkers. The results of this sampling strategy can be used to direct the focus of safety training as well as highlight deficiencies on the part of management. As a tool to reduce risk on a pillaring section, this strategy is well suited and can be very effective when used to its potential. 


\section{CHAPTER 6. SUMMARY, CONCLUSIONS, AND RECOMMENDATIONS FOR FUTURE RESEARCH}

\section{Summary}

The objective of this research was to analyze pillaring practices in Central Appalachia and, based on the analysis, develop a risk-management-based strategy for improving pillaring safety through training and intervention. From the inception of this research, it was known that, in order to reduce pillaring risk, it would be necessary to recognize that risk comes in multiple forms. The two most significant forms of risk on a pillaring section are geotechnical-based risk and personnel-performance-based risk, as shown in Figure 6.1.

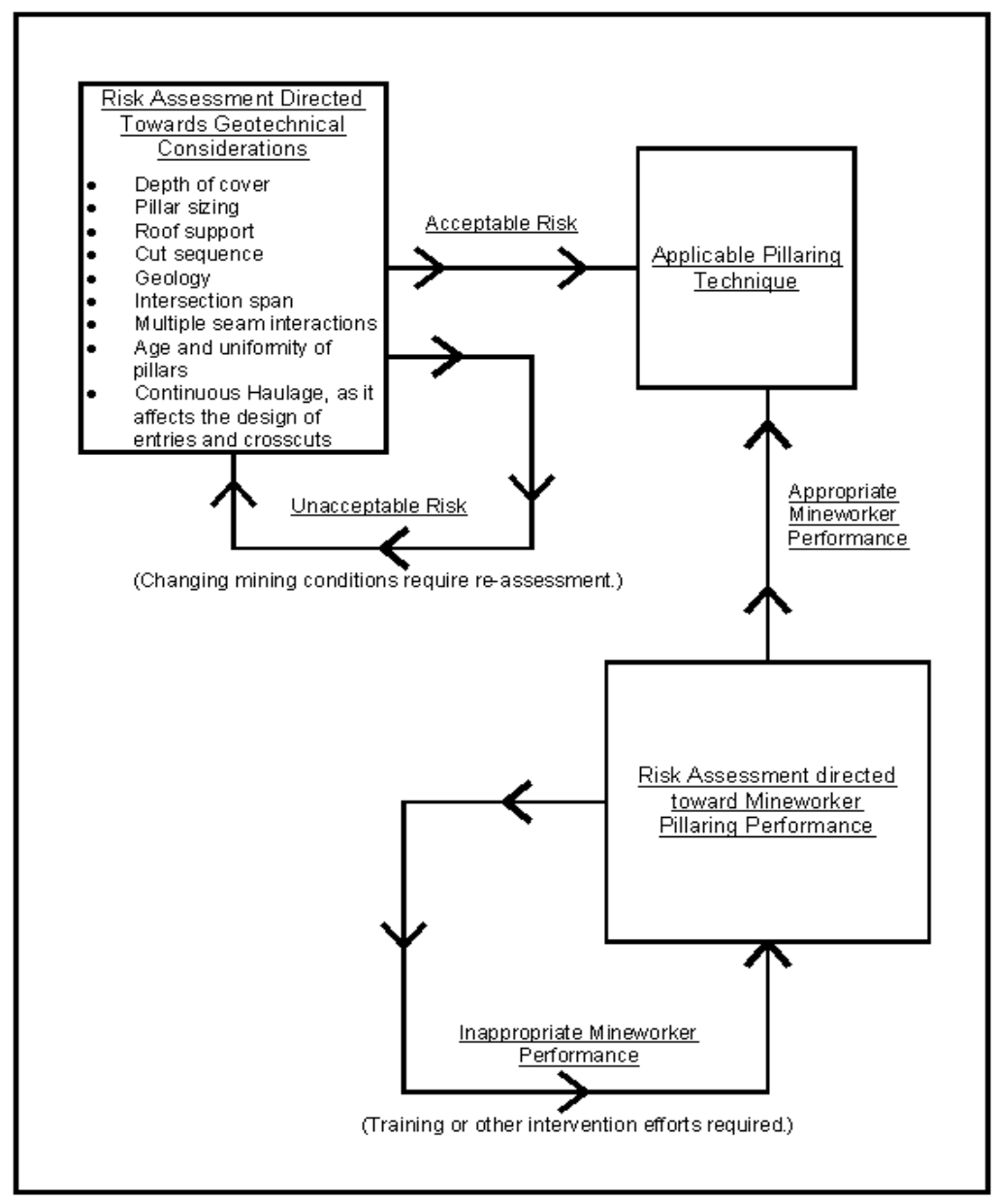

Figure 6.1 Risk Management Flowchart

Therefore, in order to improve safety, engineers and safety managers must attack risk from two sides. Prior to this thesis, the majority of the research emphasis has been directed towards engineering-related risk factors and, while it is necessary to have successful engineering-relatedrisk controls, there has been a lack of consideration for 
the residual risk that cannot be controlled through engineering methods. Regardless of the measures and risk controls, residual risk will always be present and it is left to the mineworkers to adequately cope with this residual risk. The question that had to be answered was, "How do we assess this residual risk?" The answer is through the hazard/risk-factor identification and quantitative analysis.

The first obstacle that was addressed was the identification of hazards and risk factors. This was accomplished through a combination of mine-site observations and pillaring fatality reviews. Mining is hazardous, and pillaring magnifies the particular hazard of roof and rib falls. Therefore, the roof and rib fall hazard was given precedence during this research. The risk factors that are related to the roof and rib fall hazard are both personnel-performance-based and geotechnical in nature. However, for the purpose of this thesis, emphasis was given to personnel-performance-based risk factors. During the course of the observations and reviews, correlations were made between the observed unsafe performances and unsafe performances identified in fatality reports. These recurring unsafe performances became the focus, around which the risk analysis would be structured. They are:

- unnecessary exposure to geological hazards,

- unnecessary personnel in hazardous areas,

- improper positioning of equipment operator, or helper,

- unnecessary congregation in the active mining zone, and

- unsafe positioning during downtime.

While these were the primary focus, other serious unsafe performances, not related to the roof and rib fall hazard, were also targeted due to their impact on pillaring safety. These performances include but are not limited to the following:

- horseplay,

- failure to sample air quality and quantity,

- unsafe movement or positioning with regard to machinery,

- failure to properly operate machinery, and

- failure to utilize personal protective equipment.

After the performance-based risk factors were identified, the second challenge was to measure the risk associated with unsafe performances. The solution is the Workplace Sampling Strategy developed and refined in Chapter 4 of this thesis. While the strategy does acknowledge the need for engineering or geotechnical-based risk analysis through the qualitative Pre-Mining Risk Matrix, at its core it is a quantitative analysis strategy that combines the strengths of straightforward frequency monitoring, with a sophisticated yet usable time-study approach to observation. The results yielded by the observation strategy can be utilized to improve new miner training, annual refresher training, or any other training system that is aimed at improving safety. If applied to the mining industry, it has the ability to increase safety, productivity, and improve the overall applicability of pillaring in Central Appalachia. 


\section{Conclusions}

Prior to this thesis, pillaring-risk management had primarily focused on engineering and geotechnical-based risk and, while there is an undeniable requirement for such types of risk management, there was a glaring need for a more comprehensive approach to pillaring-risk management. By shifting focus to personnel-performancebased risk, this thesis has successfully contributed to the broadening of pillaring-risk management. The success of this research and the performance-based sampling strategy that has been developed is evident by the results gathered during the field applications of the sampling strategy. By executing the sampling strategy, the observer was able to identify all but one of the risk factors listed in the previous section. These risk factors were not only identified, but also quantified. Some risk factors, such as "Horseplay" and "Failure to Warn," are only brief in nature and lend themselves only to frequency monitoring; other risk factors such as "Lingering in the Active Mining Zone" or "Improper Operator Positioning", lend themselves to both position and time monitoring. The flexible and comprehensive structure of the sampling strategy facilitated the accurate recording of both types of risk factors.

The results of the field applications successfully facilitated the identification of patterns of performance both safe and unsafe. If the sampling strategy developed in this thesis is properly executed, safety managers will be provided with a powerful tool to enhance training. By increasing the effectiveness of safety training, as well as identifying the need for immediate intervention, this strategy has the ability to improve safety and contribute to the coal mining industry's continued growth and development through safer and more productive pillaring.

\section{Future Research}

In order to continue improving safety on pillaring sections, it is imperative that efforts continue to be directed toward risk reduction. This thesis was focused on personnel-performance-based risk management. However, there are other areas where continued research effort should be directed, such as:

- improvement of remote systems to incorporate digital displays onto the remote control unit,

- recognition of pillaring-related accidents by MSHA

\section{Improved Remote Control Systems}

Current remote-control systems enable operators to run their machines from virtually anywhere within the line of sight. This ability to move the operator farther outby can have significant benefit in terms of minimizing hazard exposure. However, operating a machine does not only entail the movement and engagement of systems, it also 
requires monitoring the machine's condition, as well as the environment surrounding the machine. This is where the benefit of remote systems is lost. In many cases, water sprays, and dust from cutting obscure visibility and makes the monitoring of critical gauges, such as load gauges on the MRS units and methane displays on the continuous miner, nearly impossible The same conditions can also obscure the continuous miner operator's vision of the cutter-drum height. When the operator's vision is limited, the tendency is to move closer to the machine and, in turn, closer to more high-risk areas. The possible solution to this problem may be the integration of machine monitoring systems directly on the operator's remote. For example, if the MRS operator was able to monitor the loading on the MRS units from a safe distance outside the active mining zone, it would not only give the operator something to do during the time between repositioning, but it would also take the responsibility of monitoring the MRS loading off the continuous-miner operator and helper and not require them to venture close to the units to read gauges. In order to move the continuous-miner operator further outby, remote video could be transmitted wirelessly to a small screen integrated in the operator's control box that would provide an even better vantage point than if the operator was standing inby the discharge boom.

Robust, visual systems, necessary for mining applications, are commonly utilized in the military and defense sector; transitioning this technology for mining applications could prove to be very valuable.

\section{Recognition of Pillaring-Related Accidents by MSHA}

During the course of this thesis, an attempt was made to statistically analyze pillaring-related accident trends. However, after reviewing MSHA records, studying the MSHA accident recording template (MSHA Form 7000-1), and speaking with MSHA inspectors, it became evident that, in the event of an non-fatal accident investigation, there is no designated place on the accident recording form (7000-1) to indicate if the section was developing or retreating. Since non-fatal accidents are far more common than fatalities, having accurate and more descriptive pillaring accident data could prove valuable for future pillaring research. 


\section{REFERENCES}

Air Land Sea Application Center. 2001, "Risk Management: Multiservice Tactics, Techniques and Procedures for Risk Management"

Anonymous, 2002, "Risk Management Planning, Guidelines for Failure Mode and Effects Analysis for Automotive, Aerospace and General Manufacturing Industries," Dyadem Press, Richmond Hill, Ontario, Canada

Bilal M. Ayyub, 2005,"The Engineering Handbook Second Edition," CRC Press LLC

Bird, F. and G. Germain, 1990," Practical Loss Control Leadership", Institute Publishing, Georgia

Bise, C., 2007, West Virginia University Department of Mining Engineering, Personal Communication

Bise, C., A. Masutomi and S. Chatterjee, 1993, "Coal Mine Injury Analysis: A Model for Reduction through Training," United States Bureau of Mines, in cooperation with the Pennsylvania State University

Blake, M., 2010, West Virginia Geological and Economic Survey, Personal Communication

Brauer, R., 2005, "Safety and Health for Engineers, Second Edition," John Wiley \& Sons, Inc., Hoboken, New Jersey

Brnich Jr., M., and L. Mallett, 2003, "Focus on Prevention: Conducting a Hazard Risk Analysis," National Institute for Occupational Safety and Health, NIOSH Publication No. 2003-139

Chase, F, C. Mark, and K. Heasley, 2002, "Deep Cover Pillar Extraction in the U.S. Coalfields," National Institute for Occupational Safety and Health, West Virginia University Department of Mining Engineering

Covello,V., and M. Merkhofer , 1993, " Risk Analysis Methods-Approaches for Assessing Health and Environmental Risks," Plenum Press, New York

Cox, S., and R. Tait, 1998," Safety, Reliability and Risk Management - An Integrated Approah (2nd Edition)," Butterworth-Heinemann, Oxford, United Kingdom.

FEMA, 1993," Emergency Management Guide for Business and Industry"

Hester, R. and R. Harrison, 1998," Issues in Environmental Science and TechnologyRisk Analysis and Risk Management," The Royal Society of Chemistry Turpin Distribution Services Limited Blackhorse Road Letchworth Herts SG6 1HN, UK 
Howe, L., 2008, J. H. Fletcher \& Company, Personal Communication

Iannacchione, A. and M. DeMarco, 1992," Optimum Mine Designs to Minimize Coal Bumps: A Review of Past and Present U.S. Practices," U.S Bureau of Mines, NIOSHTIC-2 No. 10011291

Kauffman, P., S. Hawkins and R. Thompson, 1981," Room and Pillar Retreat Mining. A Manual for the Coal Industry," U.S Bureau of Mines, IC 8849, NIOSHTIC-2 No. 10002101

Kolluru, R., S. Bartell, R. Pitblado, and S. Stricoff, 1996,"Risk Analysis and Management Handbook," McGraw-Hill, Inc, New York

Lassetier, W., 2010, Virginia Department of Mines, Minerals and Energy, Personal Communication

Lawall, C., A. Ivan and H. Kennedy, 1929, "Mining Methods in West Virginia," Morgantown, WV. West Virginia University

Mark, C., F. Chase and D. Pappas, 2003, "Reducing the Risk of Ground Falls During Pillar Recovery," National Institute for Occupational Safety and Health, NIOSHTIC-2 No. 20026085

Mark, C., F. Chase and D. Pappas, 2007, "Analysis of Multiple Seam Stability," Proceedings of the $26^{\text {th }}$ International Conference on Ground Control in Mining, Morgantown, WV, pp 5-18

Mark, C., F. Chase. and R. Zipf Jr, 1997, "Preventing Massive Pillar Collapses in Coal Mines", National Institute for Occupational Safety and Health, Information Circular 9446

Mark, C., G. Karabin , J. Zelanko, M. Hoch and F. Chase, 2002, "Evaluation of Pillar Recovery in Southern West Virginia," National Institute for Occupational Safety and Health, NIOSHTIC-2 No. 20023134

Mark, C. and J. Zelanko, 2005, "Reducing Roof Fall Accidents on Retreat Mining Sections," National Institute for Occupational Safety and Health, NIOSHTIC-2 No. 20029183

Mark, C. and J. Zelanko, 2001, "Sizing of Final Stumps for Safer Pillar Extraction," Proceedings of the 20th International Conference on Ground Control in Mining. Morgantown, WV: West Virginia University, 2001 Aug; 59-66

Mines Rescue Board, 1999,"Emergency Preparedness and Mines Rescue," Mines Rescue Service, New South Wales, Australia 
Molinda, G., C. Mark, E. Bauer, D. Babich and D. Papas, 1998, "Factors Influencing Intersection Stability in U.S. Coal Mines," Proceedings of the $17^{\text {th }}$ International Conference on Ground Control in Mining, Morgantown, WV, pp 267-275

MSHA, 2005," Slam Risks" Mine Safety and Health Administration, Instruction Guide Series IG 87

MSHA , 2005," Use of Mobile Roof Support (MRS) Units for Retreat Mining" Mine Safety and Health Administration, Program Information Bulletin No. P05-19

National Academy of Engineering, 2004," Accident Precursor Analysis and Management," The National Academy Press, Washington, D.C.

Petts, J., 1998," Risk Management and Communication: Decision-making and Risk," Elsevier, Charm, Loughborough University, UK

Rowe, W., 1977, "An Anatomy of Risk." Wiley, New York

Schroder, J. Jr., 1981," Elements of Practical Coal Mining," New York City: American Institute of Mining, Metallurgical, and Petroleum Engineers, Inc. http://www.onemine.org (accessed January 15, 2009)

Stefanko, R., 1983,"Coal Mining Technology: Theory and Practice," New York City: American Institute of Mining, Metallurgical, and Petroleum Engineers, Inc.

United States National Infrastructure Protection Center, 2002,"Risk Management: An Essential Guide to Protecting Critical Assets"

Weisenfluh, J., 2010, Kentucky Geological Survey, Personal Communication 


\section{APPENDIX A}

MSHA Fatality Reports 


\title{
Fatality Case Study No. 1
}

\begin{abstract}
On Tuesday, July 15, 1997, at about 8:45 a.m., a multiple fatal roof fall accident occurred in the 4 Mains section (MMU 002-0) of Sea "B" Mining Company's Big Creek Seaboard No. 2 Mine. The accident resulted in the death of Chris Jeffers, Section Foreman, and William Cox, Continuous Mining Machine Helper.
\end{abstract}

The accident occurred during second mining as a right-side cut of coal was being mined in the pillar block between Nos. 2 and 3 entries, utilizing a remote control continuous mining machine. Near the completion of this cut, the mine roof in the crosscut immediately outby and to the right of the No. 2 entry, began working. Aware that a roof fall was imminent, Jeffers ordered everyone in the immediate area to retreat and to tram the shuttle car and continuous mining machine outby the intersection. While Jeffers and Cox were retreating, the mine roof in the intersect collapsed crushing them under the fallen roof material. The roof fall measured approximately 20 feet in width, 140 feet in length, and six to nine feet in thickness. The roof fall extended from approximately the left ribline in No. 2 entry to the left ribline in No. 4 entry. This area of the mine was developed in August 1985.

The presence of a large roof anomaly (slip) was a contributing factor to the massive roof fall. Although the slip had been detected by crew members, it was not determined that it extended through the connecting crosscuts of the Nos. 1 , 2 , and 3 entries thus presenting a much greater danger than other slips existing throughout the mine. Even though the section foreman had taken steps to install additional supplemental roof support, the large amount of roof affected by the slip and the hazard it presented, was underestimated. The roof fell suddenly and, to a large degree, in a solid mass overriding the supplemental support.

\section{GENERAL INFORMATION}

\section{General Information}

The mine is located in Tazewell County, Virginia. The mine was opened December 1973, with nine drift openings into the Lower Seaboard Coal Seam. Mining height averages 50 to 72 inches. The mine floor elevations ranged from 2170 to 2590 feet. The underground mine extends over an area of approximately 733 acres. 
Employment is provided for 61 persons working underground and seven working on the surface. The mine operates one continuous mining section (MMU-002), producing coal two shifts a day, seven days a week. The production shifts overlap, working ten hours each on afternoon and midnight. The day shift works eight hours performing maintenance and dead work. The mine produces an average of 980 tons of clean coal daily.

\section{Mining Methods}

A room-and-pillar system of mining is employed using a Joy 1410 remote control continuous mining machine, Joy 21 shuttle cars, and a Fletcher DDO-15 roof bolting machine. Coal is transported to the surface by a belt conveyor system. A trolley powered track haulage system is used to transport employees and supplies in and out of the mine. The operator is retreating the 4-Mains area of the mine utilizing a 3-cut pillar plan. The entries and rooms are numbered from left to right for identification purposes.

\section{Federal Mine Inspections}

A MSHA safety and health inspection (AAA) was completed on June 16, 1997. Another MSHA safety and health inspection was ongoing at the time of the accident but no MSHA inspection personnel were present at the mine.

\section{Roof Support}

The roof-control plan in effect was approved by the MSHA District Manager on September 7, 1993. A supplement to the plan which permitted mining 35-foot deep cuts during pillar recovery of blocks developed on 70' by 70' or larger centers was approved on June 23, 1997, and implemented at the mine on July 14,1997 . The roof-control plan requires 48 -inch minimum length resin-grouted bolts to be installed on a 4-foot maximum lengthwise and crosswise spacing. Maximum entry and crosscut widths are limited to twenty feet, with the exception of the combination belt-track entry that is permitted to be mined a maximum width of 22 feet. The maximum cut depth is 30 feet during development and 35 feet during pillar recovery. Entry and crosscut centers are specified as ranging from 45 to 100 feet.

The coal pillars are recovered utilizing a three-cut sequence with two lifts driven opposite, left and right from the entries with the third cut driven in the bottomend of the block from the crosscut. When mining 35-foot cuts a 10-foot minimum fender is required at the outby corners of the pillar block and a 6-foot fender when pillar lifts are driven 30 feet or less. All posts are required to be set on 4-foot centers. The roof-control plan stipulates that when adverse roof conditions are encountered, continuous mining machine cut depth shall be limited to 20 feet or less to provide for effective roof control.

The Lower Seaboard coal seam ranges in height from 4 to 6 feet. The immediate roof typically consists of a firm shale or sandy shale with a main roof comprised of sandy shale. The maximum overburden is 1100 feet. 
Violations of the roof control plan observed during the investigation were determined to have not contributed to the accident and the associated citations were issued under a separate inspection event.

\section{Mapping}

The mine map available at the mine on the day of the accident was up-to-date with temporary notations. Observation of the map relative to the mining that had been completed indicated that all advance and retreat mining had been marked on the map.

\section{Training Program}

The training and retraining plan to meet the requirements of 30 CFR Part 48 was approved by the MSHA District Manager on June 20, 1988. The program for training and retraining of certified and qualified persons and for training and retraining selected supervisors was approved on June 19, 1997. A review of the training records and interviews with miners indicated that all required training had been conducted.

\section{Emergency Medical Assistance}

Arrangements had been made with Quality Ambulance Service for emergency medical assistance. Following the accident, Quality Ambulance Service responded to the call. The servicing unit is located approximately 5 miles from the mine.

\section{THE ACCIDENT, RECOVERY, AND INVESTIGATION}

\section{Description of Accident}

On Tuesday, July 15, 1997, the third shift (midnight) production crew, consisting of 10 underground miners entered the mine at 12:01 a.m., under the supervision of Chris Jeffers, Section Foreman. Upon arriving on the section at approximately 12:20 a.m., Jeffers met the section foreman from the second shift, at the section coal belt conveyor feeder and discussed the pillar mining conditions on the section. The crew proceeded to their job sites and began their assigned tasks. William Cox, Continuous Mining Machine Helper and Mike Leslie, Continuous Mining Machine Operator, traveled to the No. 4 entry where the remote control continuous mining machine was parked at the left pillar cut. The cut of coal had not been completed by the previous shift. Leslie finished mining this cut and then proceeded to mine a cut in the adjacent right pillar off the No. 4 entry. Brad Joelton, Roof Bolting Machine Operator/Timberman, and Mitch Kemper, Roof Bolting Machine Operator/Timberman, were instructed by Jeffers to install the required breaker and turn posts as mining advanced across the pillar line on the section from the No. 4 entry to the No. 2 entry. 
During this time frame, Jeffers made an examination of the pillar line and detected a crack in the mine roof in the outby right side edge of the intersection in the No. 3 entry and a crack in the inby edge in the intersection in the No. 2 entry. Jeffers notified Cox and Leslie of the cracks and told them to be careful. As mining progressed, the bottom-end cut, between Nos. 4 and 5 entries, was completed and the mining machine was moved to the No. 3 entry. Mining progressed in the No. 3 entry without incident.

The left, right, and bottom-end (between No. 3 and 4 entries) cuts were mined and upon completion, the mining machine was trammed to the No. 2 entry. Joelton and Kemper had installed and precut posts for installation as mining had advanced to the No. 2 entry. Two additional posts were installed near the inby edge of the intersection in the No. 2 entry where the crack was detected. Cox began mining the right cut in the pillar block between Nos. 2 and 3 entries. During mining, the breaker posts that had been installed in the crosscut along the left rib-line at the No. 3 entry intersection, began taking weight and were beginning to crack. At this time Jeffers, returning from the belt conveyor feeder, traveled to the area where the breaker posts were taking weight. After monitoring the area, Jeffers returned to the No. 2 entry and instructed Rick Bailles, Scoop Operator, to obtain additional posts, from the section supply station. As mining continued, Jeffers, Leslie, and Joelton were located in the crosscut to the right of the No. 2 entry near the two additional posts and were monitoring the mine roof. Cox, operating the continuous mining machine by remote control, was standing on the right side of the No. 2 entry inby the intersection, and was waiting for another shuttle car to arrive. As Walter Jenkins, Shuttle Car Operator, returned for a load of coal, and was positioning his shuttle car under the boom of the continuous mining machine, Jeffers realized that the mine roof in the crosscut was beginning to fall. He shouted for everybody to get out of the area and for Cox to tram the continuous mining machine out of the cut. Kemper, who was standing between the breaker posts in the left crosscut of the No. 2 entry, immediately traveled outby the intersection to safety with Leslie, Jenkins and Joelton. The mine roof in the intersection and the crosscut to the right collapsed crushing Cox and Jeffers under the fallen roof material.

Recovery efforts were begun immediately by the midnight crew members and the day shift crew members, who had just arrived on the section. Mine officials on the surface were notified of the accident and were requested to provide additional aid. Jeffers was recovered at 9:45 a.m. and Cox recovered at 10:40 a.m. The Mine Safety and Health Administration and Virginia Department of Mines, Minerals, and Energy were notified of the accident and assisted in the recovery effort.

\section{Activities of MSHA Personnel}

Wayne Hart, Supervisory Coal Mine Safety and Health Inspector, MSHA

Richlands Field Office, was notified by telephone of the accident at 9:00 a.m. on 
July 15, 1997 by Mike Wright, Superintendent. MSHA inspectors were immediately dispatched to the mine and assisted in recovery operations. A $103(\mathrm{k})$ order was issued upon arrival of MSHA personnel to ensure the safety of any person in the coal mine until an examination and/or investigation was made to determine that the mine was safe. MSHA personnel assisted in the recovery of William Cox and provided hydraulic jacks specially maintained at the Richlands Field Office to facilitate rescue and recovery of miners trapped during roof falls.

\section{Accident Investigation}

The accident investigation began on Tuesday, July 15, 1997. Robert L. Crumrine, Assistant District Manager, District 3, was appointed as the Chief Investigator. MSHA personnel participating in the investigation were Nelson T. Blake, Chief, Roof Control Section, District 3, Joseph R. Yudasz, Coal Mine Safety and Health Inspector, District 3, Joseph A. Cybulski, Supervisory Mining Engineer, Technical Support, and Jarvis Smith, Mine Safety and Health Specialist CMS\&H.

The investigation team met at the MSHA office in Richlands, Virginia on July 15, 1997, and was briefed by District 5 personnel on the accident and the recovery of the victims. A joint meeting was held on July 16, 1997, with the Virginia Department of Mines, Minerals, and Energy discussing the procedures for the investigation. The team then proceeded to the mine where a meeting was conducted with the mine operator's management personnel and the representative of the miners' concerning the accident investigation. At the conclusion of the meeting, all personnel involved in the investigation prepared to go underground to the accident site.

After arriving on the 4 Mains section, an examination of the section and the area surrounding the roof fall was conducted. Photographs and sketches were taken of areas relevant to the accident site. The roof fall occurred in the crosscuts from the No. 2 entry to the No. 4 entry, located approximately 130 feet inby survey station 5862. The fall measured 140 feet long, 20 feet wide, and six to nine feet high.

The underground investigation was conducted in all accessible locations of the roof fall area and the 4 Mains (MMU 002-0) Section. The accident site and section were mapped and the location of the victims, equipment used, and roof support materials were plotted.

The investigation team conducted 18 voluntary interviews with employees of the mine on July 17, 1997. The interviews were conducted at the Richlands, Virginia MSHA office. All of the interviews were recorded and transcribed by a court reporting service with the exception of three mine management employees who requested that their interviews not be recorded. Copies of the 
transcripts were made available to each interested party. On July 23, 1997, follow-up interviews with six mine employees were conducted at the mine site.

\section{FINDINGS OF FACT}

\section{Physical Factors Involved}

The investigation revealed the following factors relevant to the occurrence of the accident:

1. The roof fall accident occurred July 15, 1997 at approximately 8:45 a.m. on the 4Mains (002) Section in the crosscut from No. 2 to No. 4 located approximately 130 feet inby survey station 5862 . The fall measured approximately 140 feet long, 20 feet wide, and 6 to 9 feet high. The fallen material was composed of gray laminated shale which predominately remained intact. The inby side of the fall, adjacent to the inby rib-line of the crosscut 2 to 3 , revealed the presence of a slip oriented at about a 45-degree angle into the roof. The outby side of the fall, adjacent to the outby rib-line of the crosscut 2 to 3, appeared rough and jagged as though it had broken from the weight of the roof rock cantilevering from the inby slip.

2. The area of the mine where the roof fall occurred was developed in August of 1985. The roof was supported with 48-inch resin grouted roof bolts installed on 4foot centers. The mining height at the accident site was 6 to $6-1 / 2$ feet. The overburden at the accident site was approximately 415 feet. The active working section had previously encountered many slips in the mine roof and according to the unified testimony of the miners interviewed these slips were successfully controlled by the addition of supplemental support or by avoidance based on their experience and judgment with the prevailing mine conditions.

3. Pillar blocks were being mined from right to left across the section using a threecut pillar sequence. The first cut of the sequence was mined to the left of the entry with the second cut mined opposite from and to the right of the first. The third cut would be mined from the crosscut at the bottom-end of the pillar block and intersect the number two cut. The sequence was repeated until the entire row of blocks had been second mined. The row of blocks at the accident site, was the first area of the mine where the 35-foot cut depth was used during retreat mining. However, it was the consensus of the investigating team that the depth of cut had no bearing on the accident. At the area of the accident, three pillar blocks between the number one and the number two entries were left intact to protect a seal.

4. The third shift production crew began pillar recovery mining operations in the No. 4 entry (No. 3 coal pillar). Three cuts were completed from the No. 4 entry (No.3 coal pillar-left cut; No. 4 coal pillar-right cut and bottom-end cut). 
5. According to the interviews, Chris Jeffers, Section Foreman, had examined the pillar line and detected cracks in the mine roof in the crosscut between the Nos. 2 and 3 entries while mining was in progress in the No.4 entry. The crack identified in the No.3 entry was located approximately 130 feet inby survey station no. 5861 . The crack identified in the No.2 entry was approximately 150 feet inby survey station no. 5862.

6. After mining was completed in the No. 4 entry, three pillar cuts were mined in the No. 3 entry (No. 2 coal pillar-left cut; No. 3 coal pillar-right cut and bottom-end cut). Jarvis Leslie, Continuous Mining Machine Operator, stated that he mined the left cut in the No. 2 pillar block to a depth of about 35 feet. According to Leslie and Kenneth Kemper, Roof Bolting Machine Operator, the crack observed in the No. 3 entry was located parallel to the crosscut on the outby side of the intersection. Kemper stated he observed a wedge in this crack near the right ribline of the No. 3 entry and that Jeffers had checked the wedge for roof movement during mining in this area. Kemper also stated that Cox, Continuous Mining Machine Helper, was afraid of this area while mining was in progress and left to take his lunch break. Leslie mined the bottom-end cut (No. 3 coal pillar) and positioned himself outby the crack. Leslie and Kemper stated that additional timbers had been set prior to mining in this area.

7. Upon completion of mining in the No. 3 entry, the continuous mining machine was moved to the No. 2 entry. Information from mine personnel revealed that the following posts were installed prior to mining the right cut in the No. 2 coal pillar:

A. Two rows of breaker posts and one row of turn posts were installed across the No. 2 entry inby the location of the pillar-cut (No. 2 coal pillar-right cut).

B. Twelve posts were set in the intersection, across the mouth of the crosscut 2 to 1.

C. Two rows of breaker posts were set across the crosscut 2 to 3 , adjacent the left rib-line of the No. 3 entry.

D. A row of turn posts had been set in the crosscut 2 to 3 in preparation for mining the bottom-end cut in the No. 2 pillar.

E. Two posts were set at the inby rib-line of the crosscut 2 to 3 , just outby the pillar-cut (No. 2 coal pillar-right cut) which was the approximate location from where the continuous mining machine was being operated (Cox's location prior to accident).

Leslie stated that one of these posts was real big and that he told Cox that if it breaks this--you better get out. 
8. The crack in the mine roof observed in the No. 2 entry was located on the inby side of the intersection adjacent to the left rib-line of the crosscut 2 to 3 (immediately outby the No.2 coal pillar-right cut). Leslie was performing the duties of miner helper at this time with Cox operating the continuous mining machine. Leslie stated that he placed a wedge in the crack and that the crack appeared to be an old break and not fresh.

9. Information from mine personnel indicated that mining had been in progress in the No. 2 coal pillar-right cut for approximately 25 minutes prior to the roof fall.

10. Just prior to the accident there were six persons in the area where the roof fall occurred located as follows:

A. Cox was located in the No. 2 entry near the outby left corner of the No. 2 coal pillar between the continuous miner boom and the coal rib,

B. Jenkins, Shuttle Car Operator, had positioned his empty shuttle car under the continuous mining machine's conveyor boom in position to load coal,

C. Jeffers, Leslie, and Joelton were standing near the location of the two posts installed on the inby side of the intersection and just outby the pillar cut being mined, and

D. Kemper was located between the twelve posts installed across the crosscut 2 to 1.

11. At the time of the investigation, the continuous mining machine was visible inby the roof fall. The fallen material was located approximately 14 feet outby the machine. The depth of cut was estimated to be approximately 20 feet (the right pillar-cut had holed-through into the left cut).

12. At the accident site, the crosscut No. 2 to 1 and the No. 1 entry had been filled with gob to within a couple feet of the roof.

\section{Enforcement Actions}

There were no violations of $30 \mathrm{CFR}$ observed during the investigation which contributed to the cause of the accident.

A $103(k)$ Order No. 3531716 was issued on July 15, 1997, to ensure the safety of the miners until an investigation into the cause of the accident could be concluded. A revision of the roof control plan was submitted to the District Manager and approved on July 25,1997 , prior to the termination of the $103(\mathrm{k})$ order. The revision required that nine 6-foot resin bolts would be installed in all intersections prior to second mining and that a crib would be set in conjunction with the breaker posts in the crosscuts. 


\section{CONCLUSIONS}

\section{Conclusions}

The mine roof adjacent to a large geological anomaly (slip) fell causing fatal injuries to the continuous mining machine helper and section foreman. Although the slip had been detected the connecting crosscuts by crew members, it was not determined that it extended through of the Nos. 1, 2, and 3 entries thus presenting a much greater danger than other slips existing throughout the mine. Even though the section foreman had taken steps to install additional supplemental roof supports, the sheer size of the slip and the degree of hazard it presented was underestimated. The roof fell suddenly and, to a large degree, in a solid mass overriding the supplemental support. Continual observations of the roof and breaker posts by the section foreman did enable him to sound a warning which allowed four of the six miners in the area to escape almost certain injury. 


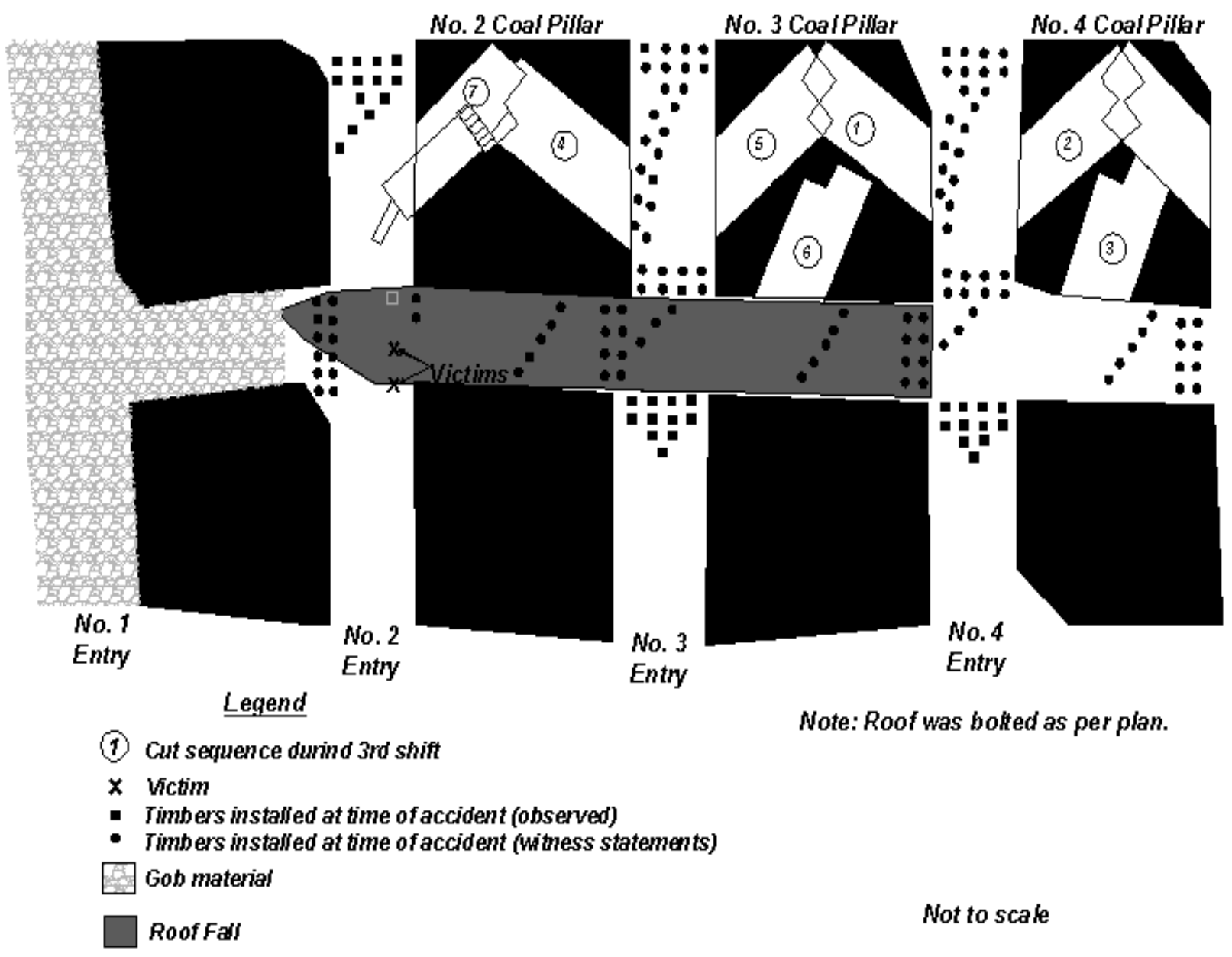

MSHA 2009 


\section{Fatality Case Study No.2}

\section{OVERVIEW}

On December 27, 2002, about 6:20 p.m., an unplanned roof-fall accident occurred on the 003-0 MMU, North Section. The accident resulted in the death of Greg Stevens, a 52 year old mobile bridge operator with 20 years of mining experience. The victim was in the intersection of the No. 5 entry as mobile roof supports (also referred to as MRS) were being repositioned. Ben Jacobs, mobile roof support operator, received serious non-fatal injuries.

The fall (estimated to be 27 feet long by 8 feet-to-18 feet wide by 1 foot-to- 6 feet thick), covered the No. 1 mobile roof support, struck the two miners and the rear of the No. 3 mobile roof support. Jacobs was removed from the mine about 7:30 p.m., and transported by the Boone County Ambulance Authority to Health Net and then to the Charleston Area Medical Center, General Division located in Charleston, Kanawha County, West Virginia. Stevens was removed from the mine about 8:30 p.m., and transported by the Boone County Ambulance Authority to the West Virginia Medical Examiners Office located in Charleston, Kanawha County, West Virginia where he was pronounced dead by the medical staff.

\section{GENERAL INFORMATION}

The mine is located near Wharton, Boone County, West Virginia. On August 5, 1994, the company began mining in the lower Winifrede coal bed. The Winifrede coal-bed, averages 60 inches in thickness, and is penetrated by 6 drift openings at the first portal and 4 drift openings at the second portal. The mine is ventilated with a 7 foot Jeffrey fan, producing 210,000 cubic feet of air (cfm) at the Candice Portal and a 5 Foot Joy fan, producing 120,000 Cubic feet of air (cfm) at the Winifrede Portal.

The mine is developed using the room-and-pillar mining system. Coal is extracted from two retreat mining sections and one advancing mining section using Joy 14-15, remote control, continuous mining machines. Coal is transported from the retreat pillar line and advancing faces by mobile bridge units, then onto the belt conveyor system which carries the coal to the surface. The mine produces approximately 5,000 tons of raw coal daily. The mine employs 114 persons, working three 8-hour shifts per day, six days per week.

Coal is produced on the day and evening shifts and maintenance is performed on the midnight shift. The underground crews enter the mine through drift openings and are transported to the working sections via track-mounted, selfpropelled, personnel carriers and self-propelled, rubber-tired, personnel 
carriers.

The immediate mine roof consists of weathered shale and sandstone with thin coal streaks The approved roof control plan specifies installation of roof bolts at a minimum of 4 -feet lengthwise and 4-feet to 5-feet crosswise spacing. This mine liberates zero cubic feet (cfm) of methane in a 24-hour period. A Mine Safety and Health Administration (MSHA) inspection (AAA) was in progress at the time of the accident.

The Non-Fatal Days Lost (NFDL) incidence rate during 2002 was 9.04 for underground coal mines nationwide and 0.00 for this mine. *

The Non-Fatal Days Lost (NFDL) incidence rate during 2001 was 7.23 for underground coal mines nationwide and 2.84 for this mine. *

* Investigation of records subsequent to the accident revealed that the operator failed to report 13 lost time accidents or illnesses for the years 2000, 2001 and 2002.

\section{DESCRIPTION OF ACCIDENT}

On December 27, 2002, about 2:30 p.m., the second shift crew, under the direct supervision of Steve Johnson, section foreman, entered the mine via trackmounted, self-propelled, personnel carriers and transferred to self-propelled, rubber-tired, personnel carriers en route to the section. Coal production started in the left pillar block of the No. 7 entry at approximately 3:15 p.m. continued in the No. 6 entry, and moved into the pillar blocks of the No. 5 entry about 5:30 p.m..

Sam Randolph, continuous-mining-machine operator, completed mining of the pillar blocks in the No. 5 entry and at about 6:10 p.m., backed the continuousmining-machine out of the right pillar block to an outby location. The process of moving the mobile roof supports out of the entry began when Greg Bartlett, mobile roof-support operator, moved the No. 3 mobile roof support from its pushout position to a location near the active pillar line in the No. 5 entry. At the time of the accident, Ben Jacobs (injured), and Bartlett were operating the four mobile roof supports located in the No. 5 entry and right crosscut. David Morgan, Seth Lewis, Kent Richards, and Greg Stevens (victim) had been operating the mobile bridge units. Randolph, Mike Donaldson, continuousmining-machine helper, Mathew Jamison, scoop operator, and Shannon Rayson, electrician, were performing other work duties on the active section.

Witnesses stated that Stevens came to the No. 5 intersection shortly before the accident and positioned himself between the No. 1 and No. 3 mobile roof supports. They also stated that they did not know why he was in that area.

Jacobs had just depressurized the No. 1 MRS when he saw the roof begin to 
fall. Recognizing that Stevens was positioned under the falling material between the No. 1 and No. 3 supports, Jacobs stated that he attempted to repressurize the No. 1 unit to stop the fall. The roof fall covered the No. 1 MRS and struck the rear of the No. 3 MRS. The fallen material covered Stevens and partially covered Jacobs. Jacobs was uncovered and removed from the fall area immediately. There was no response to attempts to communicate with Stevens.

Bartlett was located on the right side of the No. 5 entry near the No. 2 and No. 4 mobile roof supports when the roof fell, and escaped the roof fall by running down the No. 5 entry where he met Randolph.

Efforts to rescue Stevens involved moving large fallen rock by hand. Crews worked from the crosscut between the No. 4 and No. 5 entries. At approximately 6:40 p.m., rescuers found Stevens (victim) in the intersection, between the No. 1 and No. 3 mobile roof supports. A primary assessment of Stevens by the company EMT showed no vital signs. Stevens was transported to the surface at approximately 8:30 p.m. and transported by the Boone County Ambulance Authority to the West Virginia Medical Examiners Office in Charleston, Kanawha County, West Virginia where he was pronounced dead by the medical staff.

\section{INVESTIGATION OF ACCIDENT}

MSHA was notified at 7:00 p.m., on December 27, 2002, that a serious accident had occurred. MSHA accident investigators were dispatched to the mine. A 103 (k) order was issued to insure the safety of the miners until the accident investigation could be completed. The investigation was conducted in cooperation with the West Virginia Office of Miners' Health, Safety and Training (WVOMHST), with the assistance of the operator and their employees. Persons who participated, were interviewed, and/or present during the investigation are listed in Appendix A of this ..

Representatives of MSHA, the WVOMHST, miners, and company officials traveled underground to conduct an investigation of the physical conditions at the accident site. Photographs and relevant measurements were taken. Sketches and a survey were also conducted at the site. The physical portion of the investigation was completed on December 28, 2002. Interviews with persons who had knowledge of the accident were conducted on December 30 and 31, 2002, January 2 and 3, 2003, and January 21, 2003 at the Mine Safety and Health Administration, Field Office located at Uneeda, West Virginia. The investigation also included a review of training records and records of required examinations.

\section{DISCUSSION}


Training

A review of training records indicates that training had been conducted in accordance with the 30 CFR, Part 48.

\section{Examinations}

A review of records and observation of the physical evidence indicates that the required examinations had been conducted in accordance with the $30 \mathrm{CFR}$.

Physical Factors

1. The Candice 2 mine operates in the Lower Winifrede coalbed. According to the approved roof-control plan, overburden at the mine site varies from 250 feet to 550 feet and the coal seam thickness is approximately 60 inches. Coalbeds mined above the present mining operation are Buffalo Creek, 260 feet and Lower Stockton, 525 feet. Coalbeds mined below the present mining operation are the Hernshaw, 161 feet; Campbells Creek, 332 feet; No. 2 Gas, 418 feet; and Powellton, 418 feet.

2. The accident occurred while retreat mining was being conducted on the 003$0 \mathrm{MMU}$, North Section. The section had been developed by 7 entries on 60 foot centers and crosscuts angled at 60 degrees on 90 foot centers.

3. The fatal roof fall was in the intersection of the No.5 entry inby survey station No. 11518. The fall was estimated to be 27 feet long, 8 to18-feet wide, and 1 to 6 feet thick. The fallen rock covered the No. 1 mobile roof support, rear of the No. 3 mobile roof support and part of the intersection.

4. During development of this area, 42-inch fully-grouted bolts with 6 inch by 6 inch bearing plates were installed as primary roof supports.

5. A preshift examination of the 003-0 MMU, North Section was made between 5:00 a.m., and 6:00 a.m., by the 3rd shift mine examiner. Another preshift examination was conducted between 1:30 p.m., and 2:00 p.m., by the day shift examiner on the day of the accident.

6. The crew had mined both left and right in the No. 5 entry pillar blocks and were in the process of moving the four mobile roof supports outby the active pillar line when the accident occurred. Four Fletcher, model 13 \&13A, mobile roof supports were being used.

7. The mine roof at the accident scene was composed of weathered slate and sandstone. Similar roof conditions were present throughout the 003-0 MMU.

8. Entry height was about 67 inches at the accident scene. Approximately 60 
inches of coal had been mined at this location. Overburden is estimated to be 250 feet to 550 feet at the accident scene. The width of the No. 5 entry was measured to be 19 feet to 20 feet.

9. The method being used to move the mobile roof supports at the time of the accident was not in compliance with the approved roof control plan. The approved roof control plan stipulates that only one of the MRS units is to be depressurized at a time, that the units are to be moved in pairs, and when moving, each unit is to be offset not more than one-half the length of the companion unit. Physical evidence showed that the No. 1 and No. 3 mobile roof supports were depressurized from the roof at the same time. The No. 1 and No. 3 mobile roof supports were approximately 8 to 10 feet apart, one positioned in front of the other. Additionally the approved roof-control plan requires all persons to be in the clear when the mobile roof supports are raised or lowered while in the active pillar line. Physical evidence indicates that all personnel were not in the clear when the mobile roof supports were lowered. The victim and MRS operator were positioned near and/or between the units at the time of the accident.

10. Two parallel cracks in the roof of the No. 5 entry reduced the stability of the roof where the accident occurred. This instability in conjunction with the method being used to move the mobile roof supports created the hazard which caused the death of one miner and seriously injured another.

\section{ROOT CAUSE ANALYSIS}

A root cause analysis was performed on the accident. The following root causes were identified:

1. Causal factor - Unsafe mining methods were being used.

Root cause - Mine management and miners did not adhere to provisions of 30 CFR and the approved roof control plan. Personnel demonstrated a disregard for the dangers posed by conditions created when unsafe mining methods were used.

2. Causal factor - Persons worked and/or traveled under unsupported roof.

Root cause - It had become acceptable to operate and/or perform maintenance on the mobile roof supports while under unsupported roof at this operation.

\section{CONCLUSION}

It is the consensus of the investigation team that the direct causes of the fatal accident included: provisions of the approved roof-control plan were not being followed and persons worked and/or traveled under unsupported roof in the No. 5 entry. 


\section{ENFORCEMENT ACTIONS}

A 103(k) Order No. 3569006 was issued to ensure the safety of all persons in the mine until an investigation was completed and all areas and equipment were deemed safe.

A 107(a) Order No.7205663 was issued for the unsafe practices of manually tramming of the mobile roof supports, lowering more than one mobile roof support at a time, not moving the mobile roof supports in pairs, persons not being in a clear position when raising or lowering the mobile roof supports, and persons traveling and/or working under unsupported roof.

A 104(a) Citation No. 7205664 was issued for a violation of 30 CFR Part 75.202(b) stating in part that persons were traveling and/or working in the unsupported area of the active pillar line while moving the mobile roof supports.

A 104(a) Citation No. 7205665 was issued for a violation of 30 CFR Part $75.220(a)(1)$ stating in part that the operator failed to comply with provisions of the approved roof-control plan. All persons were not in the clear when the mobile roof supports were lowered.

A 104(a) citation No. 7205674 was issued to for a violation of 30 CFR Part $75.220(a)(1)$ stating in part that the operator failed to comply with provisions of the approved roof-control plan. The depressurized No. 1 and No. 3 mobile roof supports were 8 to 10 feet apart inline with each other instead of being moved in tandem, not more than one-half the length of the companion unit.

A 104(a) citation No. 7205675 was issued for a violation of 30 CFR Part $75.220(a)(1)$ stating in part that the operator failed to comply with provisions of the approved roof control plan. More than one mobile roof support unit was being lowered and moved at a time while in the active pillaring area. 


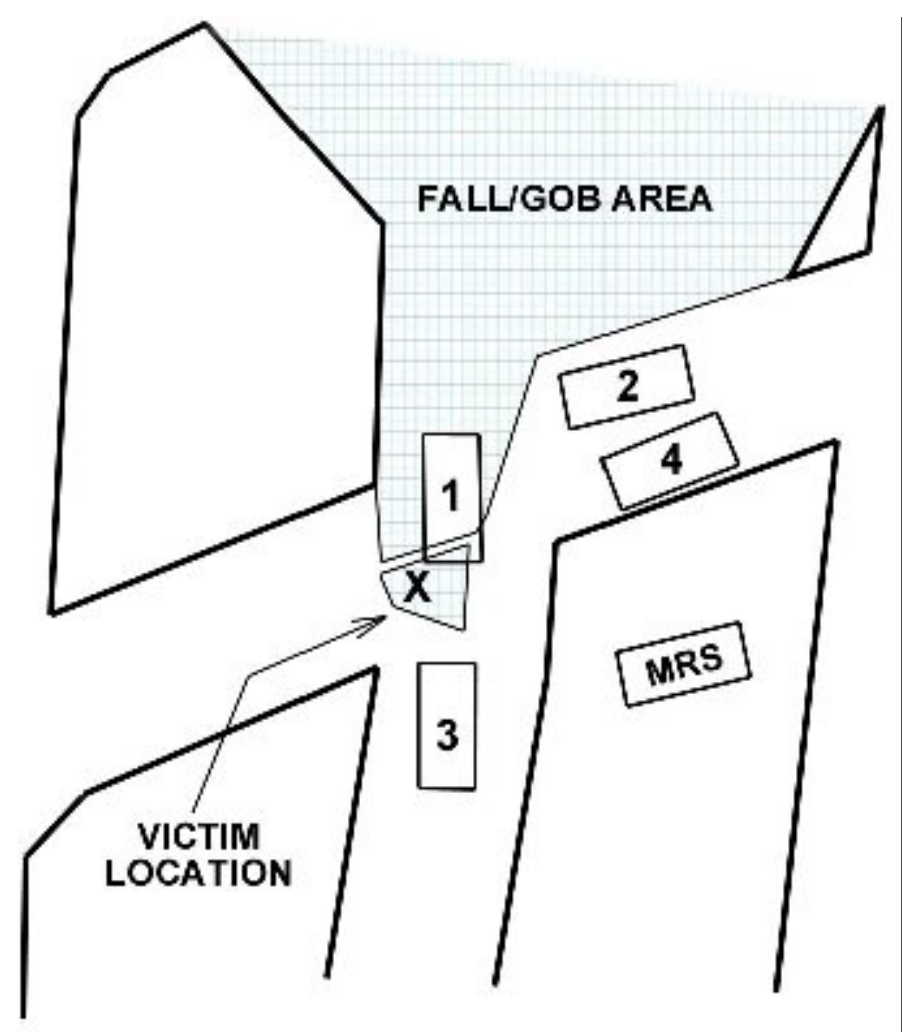

MSHA 2009 


\section{Fatality Case Study No.3}

\section{OVERVIEW}

At 3:50 p.m. on Monday August 2, 2004, Anthony Joelton, a 38-year old roof bolter operator with 14 years of mining experience was fatally injured. Joelton and the section crew had just finished setting timbers for retreat mining and were observing the roof during the final push out, when a fall of roof occurred in the No. 2 entry resulting in fatal injuries. Joelton was located in the intersection of the No. 2 entry, at crosscut No. 20, inby the turn posts. The fall ranged from 0-60 inches in thickness, 18 feet wide, and started at the center of the No. 2 entry intersection and extended inby for an undetermined distance.

The accident occurred as a result of hazardous roof conditions on the working section not being corrected. An elongated crack, parallel with the right rib, extended into the No. 2 entry intersection. A hillseam was present in the right crosscut running parallel with the No. 2 entry. The parallel joints, combined with the extraction of coal, allowed the roof fall to initiate inby the pillar line and to propagate outby to the No. 2 entry intersection at crosscut 20 . The contributing factors were: Failure to follow the approved roof control plan; the the victims position was prohibited by the provisions of the approved roof control plan. The day shift mine foreman failed to alert the oncoming shift of the hazardous condition by not recording hazardous conditions found.

\section{GENERAL INFORMATION}

The mine is located in Pinetop, Knott County, Kentucky. The mine normally operates two ten-hour production shifts per day (Monday through Thursday) with a weekend crew that works three 14-hour production shifts on Friday, Saturday and Sunday. Maintenance is performed on the third shift, Monday through Friday. Total employment is 64 persons.

Coal is extracted from the mine using a Joy continuous miner, transported to the feeder by a battery powered ramcar and placed onto a conveyor belt for transport to the surface. The coal is loaded into Caterpillar trucks and transported to the top of the mountain and dumped. The coal is next transported by truck to a preparation plant to be sized and cleaned. The finished product is then trucked or railed to the customer.

Prior to the accident, the Mine Safety and Health Administration (MSHA) completed the last regular safety and health inspection (AAA) on June 30, 2004. The mine was opened in April 2004 and had reported no injuries prior to this accident. 


\section{DESCRIPTION OF ACCIDENT}

On the day of the accident, Anthony Joelton, (the victim) reported for work at approximately 3:15 p.m. He departed the surface on a battery operated locomotive mantrip at approximately 3:30 p.m. and traveled to the end of the track. He then continued to the working section traveling in a battery powered ramcar. While Joelton was traveling to the section, the day shift crew continued pillar extraction on the 001 section. The day shift foreman, Evan Altwiller, was summoned to the No. 2 entry, by the continuous mining machine operator, for the purpose of examining a crack that ran parallel with the entry next to the right rib. The foreman observed the fracture line and checked three test holes in the immediate area. He then instructed the machine operator to back out and to resume mining from the end of the pillar block.

The second shift crew arrived on the section at approximately 3:45 p.m. Joelton proceeded to the No. 2 entry where the continuous mining machine was located. Mike Lucas, electrician, and Frank Raline, foreman, assisted Joelton in installing timbers in the No. 2 entry prior to mining the final push-out. Machine operator, Brett Daniels, checked the continuous mining machine and then trammed it into position for the final push-out. Lucas proceeded to the No. 3 entry to observe the mine roof. Within a few moments, Lucas was summoned to do some repair work. At this time Raline left Joelton and traveled to the No. 3 entry to replace Lucas and continued to observe the mine roof. Daniels loaded three cars of coal with the continuous mining machine, when, without warning, the mine roof fell. In interviews Daniels stated that he looked for Joelton but could not see him. Daniels proceeded around the tail of the machine and saw Joelton covered by rock and called immediately for Raline.

Raline immediately traveled to Joelton's location and summoned help to remove the rock. Joelton was removed to a safe location outby the fall, CPR was initiated, and Joelton was transported to the surface. Emergency Medical Personnel, on the surface, were unable to detect vital signs. Joelton was pronounced dead at the scene by the Knott County Coroner, Jeff Blair,at 6:05 p.m. August 2, 2004.

\section{INVESTIGATION OF THE ACCIDENT}

Tony Lewis, Mine Safety and Health Inspector, of District 7 Hindman field office, were notified at approximately 3:59 p.m. on the day of the accident through a telephone call from a company representative. An investigation was started the same day. An order was issued pursuant to Section 103(k) of the Mine Act to ensure the safety of miners and to secure the accident scene.

An accident investigation team was assembled and included members from District 7, and Office of Technical Support. The team immediately traveled to 
the mine, conducted a physical examination of the accident scene, interviewed persons, and reviewed training records, daily exam records, conditions, and procedures relative to the accident. MSHA conducted the investigation with the assistance of mine management and the Kentucky Office of Mine Safety and Licensing. None of the person's interviewed desired that their statements be kept confidential.

\section{DISCUSSION}

1. The accident occurred at approximately 3:50 p.m., while making the final push-out in the No. 2 entry, mining the No. 37 cut, in the right pillar block. The accident occurred at the beginning of the second shift, on the 001 working section, during retreat mining.

2. Joelton had 14 years total mining experience, 8 years and 32 weeks with the company and 14 weeks at this mine. Joelton's job title was roof bolter operator/timberman.

3. All required 30 CFR Part 48 training had been completed. The the victim was given annual refresher training on October 10, 2003, and Experienced Miner, Hazard, and Task Training for Roof Bolter Operator on April 21, 2004 for the new mine.

4. Joelton signed a weekly safety meeting attendance sheet on July $19 \& 26$, 2004, addressing among others, the topics of Pillaring and Timbering.

5. Joelton was found on the intersection side of a double row of breaker posts installed along the left rib line of No. 2 entry, in crosscut 20, inby the continuous mining machine.

6. In interviews, continuous mining operators stated that the corner of the outby pillar is typically clipped during retreat/pillar mining and supplemental support was not installed.

7. The following discrepancies between the approved plan dated April 7, 2004 and actual practices employed in the mine were identified:

A. Plan Requirement: "While coal is being cut, mined or loaded: while operating the continuous mining machine with remote control, the continuous miner operators, and other section personnel, will position themselves in a safe location outby the continuous miner, and; no personnel will advance inby the second installed row of permanent roof support outby the face." (Page 15 Number 1(a) of approved roof control plan dated April 7, 2004).

Practice: The the victim was found inby the turn posts and inby the continuous mining machine. 
8. A Joy continuous miner extracts the Hazard No. 4 seam, which averages 40 to 48 inches in height. At the time of the accident, the mine, in operation since April of 2004, was in the process of retreat mining the first developed panel (1st Left North Mains). Two additional planned panels are expected to deplete limited reserves within 6 months.

The accident occurred in the No. 2 entry at crosscut 20 intersection, near the left rib line. The the victim was positioned on the intersection side of a double row of breaker posts installed along the left rib line of No. 2 entry in crosscut 20.

9. Roof failure nearly spanned No. 2 entry, from rib to rib. The outby edge of the roof fall ended approximately halfway through the crosscut 20 intersection. The fall appeared to extend inby an estimated 50 to 70 feet, with eleven rows of bolts visible along the right rib line, protruding approximately 2 feet from the fallen material. Several bolts on the left side protruded 2 to 3 feet.

10. The fall material that struck the victim was originally located on the outby edge of the main failure zone. At the time of the investigation, it was lying on the floor in two sections, one overlapping the other, reportedly placed there by workers recovering the victim. The slab on top was roughly 2 feet by 4 feet by up to 8 inches thick. The underlying slab was 2 feet by 3-4 feet and up to ten inches thick where visible, with feathered edges. Each slab was estimated to weigh between 400 and $700 \mathrm{lbs}$.

11. The main failure occurred as two elongated blocks, separated by a steeply dipping discontinuity near the center of the entry. In crosscut 20, the fall was 4 $1 / 2$ feet thick near the center, tapering to a few inches near the right rib and roughly 3 feet near the left rib. Fall material thickness may have been greater further inby. Except for small slabs on the periphery of the fall, the main blocks, where visible, remained mostly intact after impact with the floor.

12. The discontinuity bisecting the main fall extended outby to the crosscut 19 intersection, angling slightly toward the right rib. It was dilated to a maximum of $1 / 8$ inch at crosscut 20 and became tight further outby. Roughly parallel curvilinear discontinuities were mapped in adjacent entries and were found to be persistent over several crosscuts.

13. In No. 3 entry, a discontinuity extended from the crosscut 20 intersection, curving to the left pillar line, terminating in crosscut 19 at the pillar between No. 2 and No. 3 entries. This discontinuity was heavily stained with iron oxides and had been strapped in several locations on development. The intermittent strapping indicates that the condition was recognized by mine personnel and understood to require additional roof support. Mine personnel indicated that these types of discontinuities were encountered throughout the panel. The general orientation of the discontinuities mapped underground matched those observed in surface outcrops in the vicinity of the mine portal. 
14. Exposure of discontinuities on development, some with oxide staining (indicating the presence of water), and orientations consistent with surface joints suggest that the discontinuity bisecting the fall existed prior to retreat mining and was part of a northwest oriented joint set.

15. Entry width measurements taken in No. 2 entry between crosscuts 19 and 20 were 20 feet 11 inches near the pillar notch and 20 feet 6 inches near the center of the pillar. Outby crosscut 19; No. 2 entry was 19 feet, 8 inches wide. Widths in adjacent entries were 19 feet 9 inches and 19 feet 4 inches.

16. The roof was supported with grade $60,5 / 8$ diameter, 48 -inch long fully grouted, headed rebar bolts installed on 4 foot by 4 foot centers.

17. The four turn posts required by the roof control plan to be installed diagonally across the intersection were not visible at the time of the investigation. Based on the observed position of these turn posts in nearby entries, the fall would have taken out the two turn posts furthest inby, but should not have reached the two outby posts. However, what appeared to be a post was visible under the leading edge of the fall blocks.

18. The original configuration of posts in the No. 2 entry at crosscut 20 intersection was unclear as several additional posts were set during recovery of the victim, and at least one of the breaker posts in crosscut 20 (left side) was moved to facilitate recovery efforts.

19. The immediate roof adjacent to the accident location consisted of a hard, gray, sandy micaceous shale with prominent black laminate surfaces. The fall material, observed remotely, appeared to be similar to outby observations. A localized area of immediate roof in entry one, exposed between the coal seam and the mined roof, consisted of dark gray mudstone.

20. Test samples of both rock types were subjected to immersion in fresh water for 48 hours. The integrity of the samples was unaffected.

21. Groundwater inflow into the mine is generally limited to small seeps, although development of crosscuts 15 through 20 in No. 1 entry was halted due to the presence of water.

22. Rib sloughing to a depth of a few inches occurred in No. 2 entry between crosscuts 19 and 20. Reportedly, the sloughing occurred in the interval between the time of roof failure and the investigation on the afternoon of August 3rd.

23. A double row of breaker posts had been installed in No. 2 entry on the outby side of the crosscut 20 intersection (subsequent to the fall in No. 2 entry). A few 
posts on the left side appeared to be taking weight, as evidenced by the crushing of the post wedges.

\section{ROOT CAUSE ANALYSIS}

An analysis was conducted to identify the most basic causes of the accident that were correctable through reasonable management controls. During the analysis, causal factors were identified that, if eliminated, would have either prevented the accident or mitigated its consequences. Listed below are causal factors identified during the analysis and their corresponding corrective actions implemented to prevent a recurrence of the accident:

1. Causal Factor: The victim was in an unsafe location in the No. 2 entry intersection inby the continuous mining machine.

Corrective Action: Mining practices and procedures should be reviewed to ensure that during retreat mining no one is allowed inby the continuous mining machine. The mine operator should reinstruct all affected personnel in the approved roof control plan and ensure compliance with its provisions.

2. Causal Factor: A review of examinations for hazardous roof conditions conducted during the day shift, prior to the accident, on the 001 mechanized mining unit (MMU) indicated that observed hazardous conditions were not recorded in the approved record book. In interviews the foreman acknowledged observing the crack radiating down the right rib but failed to record this condition. Identification of these conditions during the examination should have been recorded in the approved record book in order to alert the oncoming shift foreman to hazardous conditions. The record could have prompted corrective actions by mine management.

Corrective Action: The certified persons making the examinations should properly identify, make the appropriate corrections, and record all hazardous conditions. Mine management should develop and follow procedures to identify and correct any and all hazardous conditions and to notify all persons affected by the condition.

\section{CONCLUSION}

The accident occurred as a result of hazardous roof conditions on the working section not being corrected. An elongated crack, parallel with the right rib, extended into the No. 2 entry intersection. A hillseam was present in the right crosscut running parallel with the No. 2 entry. The parallel joints, combined with the extraction of coal, allowed the roof fall to initiate inby the pillar line and to propagate outby to the No. 2 entry intersection at crosscut 20 . The contributing factors were: Failure to follow the approved roof control plan; the victims 
position was prohibited by the provisions of the approved roof control plan. The day shift mine foreman failed to alert the oncoming shift of the hazardous condition by not recording hazardous conditions found.

\section{ENFORCEMENT ACTIONS}

Order No. 7547237 was issued on August 2, 2004, under the provisions of section 103(k) of the Mine Act.

A fatal accident occurred at this operation on August 2, 2004, when the miner was fatally injured by a pillar fall on the 001 active section. This order is issued to assure the safety of all persons at this operation. It prohibits all activity in the underground areas of the mine until MSHA has determined that it is safe to resume normal mining operations at this mine. The mine operator shall obtain prior approval from an authorized representative for all actions to recover and/or restore operations to the affected area.

Citation No. 7501539 104(a), S\&S, Moderate negligence was issued for a violation of $75.220(\mathrm{a})(1)$.

An investigation of the fatal roof fall accident that occurred on August 2, 2004, determined that the approved Roof Control Plan, dated April 7, 2004, was not being complied with in the No. 2 entry on the 001 Mechanized Mining Unit (MMU). The victim was found inby the continuous mining machine while the continuous mining machine was mining. The approved plan required that all personnel be located in a safe location outby the continuous mining machine when the continuous mining machine is mining.

Citation No. 7501540 104(a), S\&S, Moderate negligence was issued for a violation of $75.360(\mathrm{f})$.

An investigation of the fatal fall of roof accident that occurred on August 2, 2004, determined that the day shift foreman who conducted the pre-shift examination failed to properly record the results of his examination of the 001 Mechanized Mining Unit (MMU). The foreman stated that hazardous conditions were found and that a decision was made to back out the continuous mining machine and approach the pillar block from another position. The pre-shift record book did not contain any entries identifying the hazardous conditions. 


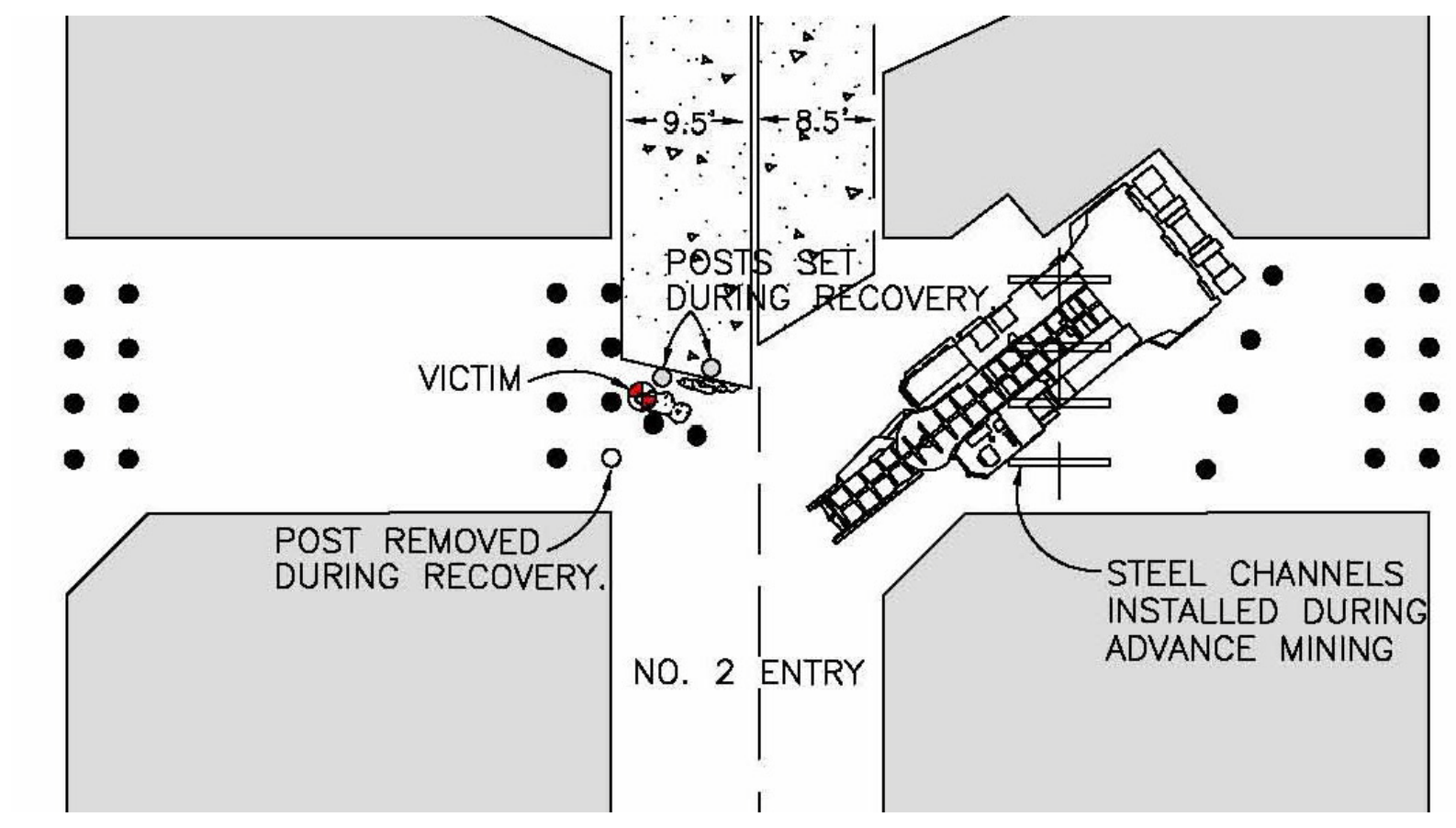

MSHA 2009 


\section{Fatality Case Study No.4}

\section{OVERVIEW}

At approximately 10:40 a.m. on Saturday, January 13, 2007, 48-year-old Thomas Jamison and 33-year-old Peter Reynolds, mobile roof support (MRS) machine operators, were fatally injured when a portion of the mine roof collapsed, pinning both individuals. Jamison and Reynolds had 12 years and 2.5 years of mining experience respectively. Jamison and Reynolds were repositioning MRS machines after the completion of the third lift in the \#3 entry while performing retreat mining on the North Section. The slicken-sided portion of the mine roof that collapsed measured approximately 8 feet in width by 9 feet in length and was up to 18 inches thick.

\section{GENERAL INFORMATION}

The mine, is located near Cucumber, in McDowell County, West Virginia. The mine began operation on February 27, 2006, and operates in the Pocahontas No. 3 bituminous coal seam which averages 90 inches in height. Miners enter the mine through slope portals via diesel-powered, rubber-tired, self-propelled personnel carriers.

The mine employs 138 persons and operates 2 continuous mining units, of which one is a developing section and one is a retreat section. Approximately 6,000 tons of raw coal is produced daily on two 9-hour production shifts per day, 7 days a week. There are 3 production crews, referred to as $A, B$, and $C$ crews. There are also two maintenance crews, referred to $D$ and $E$ crews. The mine liberates 919,243 cubic feet of methane in a 24-hour period.

The last regular MSHA inspection of this operation was completed on December 4, 2006. A new inspection was started on January 8, 2007, but the physical on-site portion of the inspection had not begun. The Mine's Non Fatal Days Lost (NFDL) incidence rate in 2006 was 9.74, compared to the national average of 5.01 for mines of the same type.

\section{DESCRIPTION OF THE ACCIDENT}

On Saturday, January 13,2007 , at approximately 6:30 a.m., the C-crew, under the direction of Bill Rickman, section foreman, traveled to the North Section (Mechanized Mining Units 001 and 002). The North Section was conducting retreat mining, or second mining, of the coal pillars which remained after initial development. After arriving on the section, Rickman and the crew met at the section power center where they had prayer and a short safety meeting. After 
the meeting, he conducted a 30 CFR 75.362 on-shift examination of the pillar line.

After the examination, Rickman returned to the \#4 entry, just outby the pillar line. He instructed the crew to mine 3-lifts in the $\# 4$ entry and then proceed to the \#3 entry. No problems were encountered during mining.

The MRS machines were moved and set-up to begin mining the coal pillars from the \#3 entry inby survey spad station 1037. Brad Crisen, continuous mining machine operator, and Patrick Evans, continuous mining machine helper, moved the continuous mining machine into the \#3 entry and extracted the first lift from the coal pillar located on the left side of the entry (pillar number 107). Next, Jamison and Reynolds moved MRS \#1 outby (away from the face) approximately 8 feet. During this time, Ryan Evans, General Mine Foreman, was on the North Section to take seam height measurements and determine if extensions were needed for the MRS machines.

Crisen and Patrick Evans moved the continuous mining machine and mined the second lift from the coal pillar located on the right side of the entry (pillar number 106). While the second lift was being mined, Ryan Evans, Rickman, Jamison, and Reynolds were located in the cross cut between the \#3 and \#2 entries just outby the blocks of coal being mined. Upon completion of the lift, Jamison and Reynolds moved MRS \#2 outby approximately 8 feet. Rickman traveled into the \#3 entry just behind Jamison and Reynolds to watch the mine roof while the MRS machines were being repositioned. Rickman normally did this while the MRS machines were being repositioned. The movement of MRS \#2 placed the outby end of the machine approximately 5 feet inby the outby corner of the second lift. The outby end of both of the mobile roof support machines were almost side-by-side.

Crisen and Patrick Evans moved the continuous mining machine and mined the third lift from the coal pillar located on the left side of the entry. The third lift was advanced approximately 7.5 feet deeper than the maximum distance allowed in the approved roof control plan. This condition did not contribute to the accident, and therefore a section 104(a) citation was issued under another inspection event.

Crisen and Patrick Evans moved the continuous mining machine outby the last open cross cut in the \#3 entry. Both MRS machines had green lights illuminated, indicating the canopies of the machines were pressurized against the mine roof. Crisen repositioned the continuous mining machine trailing cable while Patrick Evans left the area to retrieve his lunch. Ryan Evans walked from the crosscut between the \#2 and \#3 entries, and began to talk to Crisen.

Jamison and Reynolds traveled up the \#3 entry and repositioned MRS \#1. Rickman was located immediately behind Jamison and Reynolds, near the 
outby corner of lift 3. Jamison had the radio-remote control transmitter that was used to operate the MRS machines. This transmitter enabled the equipment operator to operate the MRS machines while standing in a safe location. The MRS machines trailing cables were suspended from the mine roof with single strand rubber coated \#14 copper wire attached to roof bolt plates. One of the tie wires had been twisted too tight and would not allow the MRS \#1 machine trailing cable to drop freely from a roof bolt plate.

Reynolds traveled inby to within 5 feet of MRS \#1 and untwisted the tie wire to free the machine's trailing cable. Reynolds then walked outby near Jamison and Rickman. The three miners were approximately 15 feet outby MRS \#1 and \#2. Using the remote control, Jamison released MRS \#1 from the mine roof, while the miners were positioned inby the outby corner of the previously mined third lift. This was in an effort to reposition the machine for the next lift to be mined.

At the same time, Kieth Hellems, \#2 shuttle car operator, stopped his shuttle car and walked up the \#3 entry. As Hellems walked up the \#3 entry, he saw the mine roof sagging and taking weight. At approximately 10:40 a.m. he started to signal and shout, as a portion of the mine roof fell. When the mine roof fell, Jamison and Reynolds were pinned and Rickman, whose hand was resting on the mine roof, was nearly struck. The falling mine roof caused Rickman's hand to drop away from the mine roof.

Ryan Evans traveled to the roof fall as Hellems ran outby past the continuous mining machine and \#2 shuttle car. Ryan Evans immediately instructed Crisen to reposition MRS \#3 and \#4 that were located in the last open crosscut between the \#3 and \#4 entries. Crisen moved MRS \#3 and \#4 from the \#4 side of the cross cut to the \#3 side of the cross cut and pressurized the machines against the mine roof. Ryan Evans then had Crisen to pressurize MRS \#1 against the mine roof, which was in a lowered position, 6 to 8 inches from the mine roof.

The remaining crew members traveled to the roof fall and set temporary roof supports (timbers and cribs) to ensure no additional roof falls would occur. The fallen mine roof rock was moved, the victims were recovered and transported by the McDowell County Emergency Authority and the Wideners Ambulance Service to Welch Hospital.

\section{INVESTIGATION OF THE ACCIDENT}

The Mine Safety and Health Administration (MSHA) was notified of the accident at 10:45 a.m. on Saturday, January 13, 2007 when Darrell Keene, day shift maintenance foreman called the MSHA Call Center. MSHA personnel from the Princeton, Mount Hope, and Summersville field offices were immediately dispatched to the mine site. A 103(k) order was issued to insure the safety of all persons during the investigation. The $103(\mathrm{k})$ order was later issued in writing. 
The investigation was conducted with the cooperation of the West Virginia Office of Miners' Health, Safety and Training (WVOMHST), MSHA Technical Support personnel the mine operator and the mine employees.

The accident scene was photographed, sketched, and surveyed. Interviews were conducted of persons considered to have knowledge of the facts concerning the accident. A list of the persons who participated in the investigation is contained in Appendix A. The on-site portion of the investigation was completed, and the $103(\mathrm{k})$ Order was modified on January 17,2007 , to permit normal mining operations to resume on the North Section and on the West Section.

\section{DISCUSSION}

\section{$\underline{\text { Roof Control Plan }}$}

The third cut extracted prior to the roof fall extended 7.5 feet beyond the center of the coal pillar. Page 12 of the approved roof control plan, under the subtitle, Full Pillar Recovery Plan (MRS) Deep Cut Mining, requires "The initial lifts taken from the pillar will not exceed $1 / 2$ the block width. Blocks of larger dimensions may be mined using this plan, provided cut depth does not exceed one half the width of the pillar blocks or 40 feet."

Page 12a, item 9 under the subtitle "Safety Precautions for Mobile Roof Supports" of the approved plan states, "A breakaway type hanger shall be used in the pillar line to keep persons from going inby to take the mobile roof support machine trailing cables down." Reynolds traveled to within 5 feet of MRS \#1 to take down a portion of the MRS machine trailing cable. Reynolds untwisted the tie wire which was too tight immediately before the roof fall. During interviews, it was indicated that the tie wire had been too tight on prior occasions. When overly tightened, persons would have to walk closer to the MRS machine and loosen the wire. This condition did not contribute to the cause of the accident, and therefore the condition was cited under a separate inspection event.

When the roof fall occurred, Jamison, Reynolds, and Rickman were near the center of the \#3 entry, approximately 15 feet outby MRS \#1. Page 12a, item 7 of the approved roof control plan under subtitle, Safety Precautions for Mobile Roof Supports, states, "Except when performing maintenance, all personnel shall stay in a safe area (minimum distance of 20 feet from the MRS machines) when the MRS machines are being trammed or the shields are being raised or lowered." The term "shield" refers to upper portion of the machine which contacts the mine roof. In addition, the miners were located inby the outby corner of the second lift mined from the left pillar (pillar number 107), while MRS \#1 was not in contact with the mine roof. From his position, Bill Rickman, Section Foreman, could observe the miners working in close proximity to the MRS machines and determine they were inby the previously mined area of the coal pillar. 


\section{Handling of MRS Trailing Cable}

Each MRS machine was equipped with a No. 6 AWG trailing cable which provided 480 volt electrical power to the machine. On-board each MRS machine was a cable reel which stored additional cable which facilitated the movement of the machine. Prior to the accident, the trailing cable of MRS \#1 was manually removed from twisted wire which suspended the cables from the mine roof. To manually handle the cable, the MRS operators had to walk closer to the MRS machine than the 20 -foot minimum distance specified in the approved roof control plan.

\section{Training}

An inspection of the training records indicated that Jamison, Reynolds, and all current mine employees had received newly experienced miners training in accordance with 30 CFR 48. All persons currently working on the North Mains section indicated the Full Pillar Recovery Plan (MRS) Deep Cut Mining part of the roof control plan had been reviewed. The mine operator could not provide any documentation of persons attending the review of the pillar plan.

The training was inadequate. During interviews, miners were not aware of the second sentence on page 12 of the approved roof control plan which states, while coal is being mined, all other persons shall be in a safe location outby the last open break. Miners indicated that MRS machine operators, foremen, electricians, continuous mining machine helpers, and anyone else observing mining operations stayed in the last open cross cut while mining was being performed.

This was the first occasion in which retreat mining was conducted at this mine. For that reason, required task training for retreat mining was crucial to the safety of persons exposed to the new mining method.

\section{ROOT CAUSE ANALYSIS}

A root cause analysis was conducted to identify the most basic causes of the accident that were correctable through reasonable management controls. Listed below are root causes identified during the analysis, and their corresponding corrective actions were implemented to prevent a recurrence of the accident.

Root Cause: Management allowed noncompliance with the roof control plan. Miners traveled inby a minimum safe distance of 20 feet outby the mobile roof support machines to approximately 15 feet. This was a location inby a previously mined portion of the adjacent coal pillar. While in this position, MRS \#1 was lowered so that it was no longer pressurized against the mine roof.

Corrective Action: Retreat mining provisions were removed from the approved 
roof control plan, causing the mining of coal pillars in the mine to cease. Miners and management were retrained in the provisions of the approved roof control plan to ensure they were knowledgeable in the requirements of the plan.

Root Cause: Miners exposed to retreat mining methods did not receive training in the health and safety aspects and safe operating procedures for work tasks, equipment, and machinery, and supervised practice during nonproduction. The required training and practice is essential to ensure that miners understand safe work procedures and are prepared to follow the safe work procedures outlined in the approved plan.

Corrective Action: Retreat mining provisions were removed from the approved roof control plan, and retreat mining has ceased, thereby removing miners from any exposure to hazards which may be presented during retreat mining.

\section{CONCLUSION}

James David Jamison and Peter Reynolds, mobile roof support machine operators, were fatally injured when a portion of the mine roof collapsed onto both miners. At the same time Bill Rickman, section foreman, narrowly escaped serious or fatal injuries. Jamison, Reynolds, and Rickman were allowed to travel closer than the minimum safe distance of 20 feet from the MRS machines specified in the approved roof control plan. This travel was also inby a previously mined portion of the adjacent coal pillar. While in this position, MRS \#1 was lowered so that it was no longer pressurized against the mine roof. The accident occurred because effective safe work procedures and practices specified in the approved roof control plan were not enforced by mine management. In addition, miners were not properly trained in safe work procedures for retreat mining.

\section{ENFORCEMENT ACTIONS}

1. A 103(k) Order, No. 7259197 was issued to ensure the safety of the miners until the investigation could be completed.

2. A 104(d)(1) Order, No. 7259200, was issued for a violation of 30 CFR 75.220(a)(1), stating the Mine Operator did not follow the approved roof control plan for mine ID 46-09066 on the North Mains Section (MMU-001/MMU-002). The approved roof control plan states under subtitle, "Safety Precautions for Mobile Roof Supports", page 12a, item 7, "Except when performing maintenance, all personnel shall stay in a safe area (minimum distance of 20 feet from the mobile roof support machines) when the mobile roof support machines are being trammed or the shields are being raised or lowered." Bill Rickman, Section Foreman, and two mobile roof support machine operators were not in a safe location and were located closer than the minimum safe distance of 20 feet from the MRS while the \#1 mobile roof support machine was 
being trammed with the shield lowered. A slicken-sided portion of the mine roof collapsed causing the multi-fatality accident.

3. A 104(g)(1) Order, No. 7259201, was issued for a violation of 30 CFR 48.7(a) stating, miners assigned to new work tasks as mobile roof support operators were attempting to perform those tasks without having been trained in all the safe operating procedures related to their assigned tasks.

The second sentence on page 12 of the approved roof control plan stated, "While coal is being mined, all other persons shall be in a safe location outby the last open break. Miners being interviewed stated that the mobile roof support machine operators, foreman, continuous mining machine helpers, and anyone else observing mining operations stayed in the last open cross cut while mining was being performed.

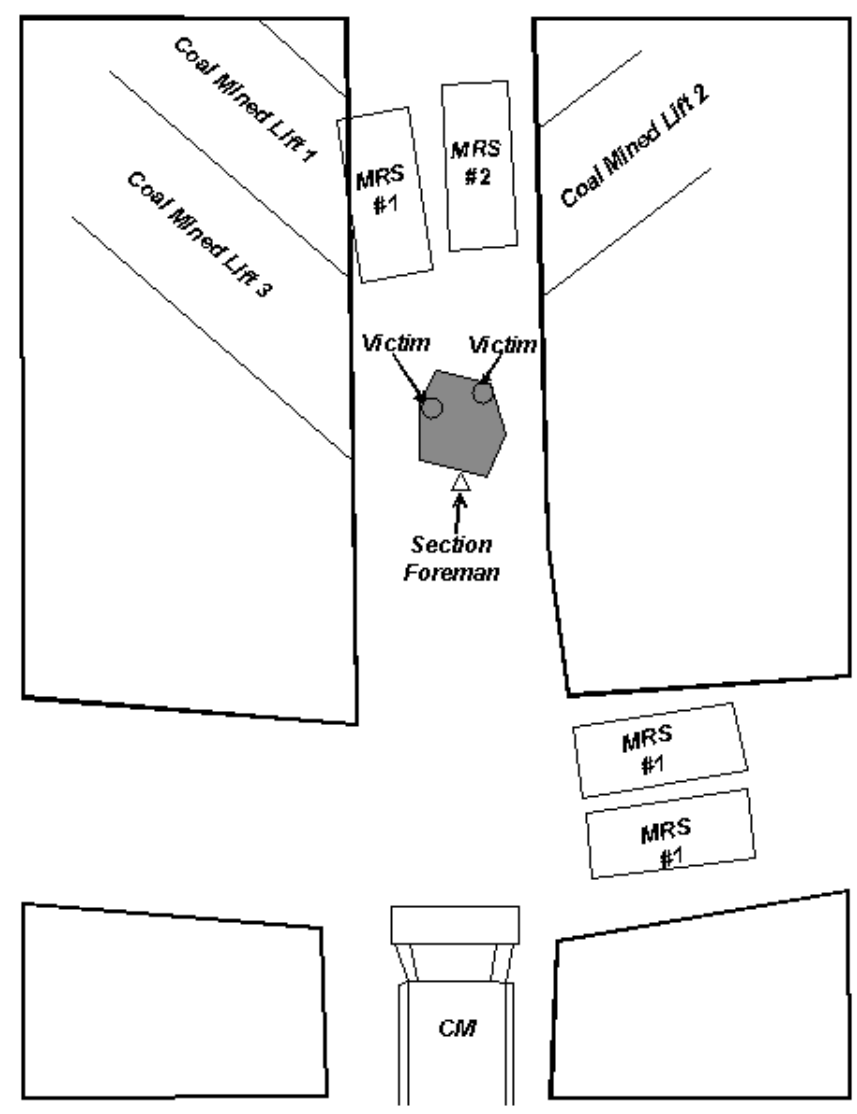

MSHA 2009 


\section{Fatality Case Study No.5}

\section{OVERVIEW}

At 9:30 p.m. on Wednesday, August 3, 2005, Clyde Russell, a 39-year old section foreman with 11 years of mining experience and William Bradford, a 23year old scoop operator with 36 weeks of mining experience were fatally injured

The second shift crew was conducting retreat mining on the 003 mechanized mining unit (MMU). After mining the final lifts of a pillar, the crew was moving the four mobile roof support units (MRS) to the next location to be mined. While they were moving the MRS units, a roof fall occurred in the intersection. Eyewitnesses reported that Russell and Bradford were last seen standing beside the No. 2 MRS inby the intersection. After the fall, workers called out to Russell and Bradford, but there was no response. Bradford's body was recovered on Thursday, August 4, 2005, and pronounced dead by Harlan County Deputy Coroner Gerald Scott at 5:30 a.m. Russell's body was recovered on Sunday, August 7, 2005, and pronounced dead by the Deputy Coroner at 7:18 a.m.

The accident occurred because of a confluence of factors, including failures to comply with the approved Roof Control Plan, training, faulty pillar recovery methods, and failure to correct hazardous conditions.

\section{GENERAL INFORMATION}

The mine is located near Cumberland, Harlan County, Kentucky. Coal is produced on the first and second shift with maintenance being conducted on the third shift. The mine produces approximately 5,000 tons of raw coal daily using the room-and-pillar method. The mine provides employment for 73 underground workers (including contractors) and three surface workers.

Coal is extracted with remote-controlled continuous mining machines equipped with flooded bed scrubbers utilizing a 40 -foot extended cut plan. Shuttle cars are used to transport coal to the section belt conveyor feeder. Coal is then transported to the surface by a series of belt conveyor flights. The mine is ventilated with a single mine fan, utilizing an exhausting system. The mine liberated 131,628 cubic feet of methane in a 24 hour period when it was last sampled.

The mine was opened into the 48 to 120 -inch thick Harlan seam by seven drift openings in 1999. As the mine developed, the parting became thicker in the coal seam, splitting the seam into the Upper and Lower Harlan seams. Mining was confined to the Upper Harlan seam until early in 2005, when three entries were ramped down to the Lower Harlan seam through an interburden of approximately 35 feet. At the time of the accident, coal was being mined on two 
sections, the 002 and 003 MMUs. The 002 MMU was advancing six entries in the Upper Harlan seam. The $003 \mathrm{MMU}$ was recovering pillars in the Lower Harlan seam, at the intersection of the 1 Left Panel and a set of submains (each of which were four entries wide).

The approved Roof Control Plan provided two sequences for recovering pillars: one for mining a row of pillars from left to right, and another for mining right to left. The maximum entry width permitted by the plan was 20 feet with a provision for 22 feet when excessive rib sloughing was encountered. Prior to this accident, the last reported roof fall occurred on June 6, 2000. Over the life of the mine, the operator recovered pillars in multiple panels in the Upper Harlan seam. However, this was the first area where pillars were recovered in the Lower Harlan seam at this mine Prior to the accident, the Mine Safety and Health Administration (MSHA) completed the last regular safety and health inspection on June 30, 2005. There was an on-going regular inspection being conducted at the time of the accident. The Non-Fatal Days Lost (NFDL) injury incidence rate for the mine in 2004 was 14.03 compared to a National NFDL rate of 5.56 for 2004 .

\section{DESCRIPTION OF ACCIDENT}

On August 2, 2005, at approximately 11:00 p.m., Shane McConnel, third shift mine foreman, instructed Brad Sheiffer, third shift 003 MMU section foreman, on the procedures for setting up the section for a projected 90-degree change in pillar recovery direction as $003 \mathrm{MMU}$ retreated out of the 1 Left Panel and into the Submains. Sheiffer understood that he was to set up the $003 \mathrm{MMU}$ so that pillars could be recovered from left to right across the submains, beginning with the last pillar on the right side of the 1 Left Panel. Later that night, Sheiffer's crew pulled the $003 \mathrm{MMU}$ power center and beltline back and started to set up the mining equipment for the change in direction. However, this task was not completed at the end of the shift.

At approximately 7:00 a.m., the first shift $003 \mathrm{MMU}$ crew entered the mine and traveled to the section. Corey Dotson, first shift 003 MMU section foreman, checked the working places. The crew positioned the MRS units and the continuous mining machine to begin pillar recovery of the last pillar on the right side the 1 Left Panel. Instead of recovering pillars across the Submains from this point, Dotson intended to recover the last row of pillars from the $1 \mathrm{Left}$ Panel, mining from right to left across the panel.

Grant Kimber, first shift continuous mining machine operator, began mining, alternating lifts to the left and right from the 1 Left Panel No. 4 entry (cuts $A$ and $B$, as shown on the Accident Site sketch on page ii). After the fourth lift (B) was mined to the right of the entry, the conveyor belt stopped. At this time they moved the continuous mining machine around the second outby block to reroute the cable and set breaker timbers in the last open crosscut between the Submains Nos. 1 and 2 entries. After the belt was started, they mined three 
more lifts (B) to the right of the No. 4 entry and two lifts straight off the inby end of the Submains No. 1 entry. Kimber mined the lifts to a maximum depth of approximately 30 feet in order to prevent draw rock from falling on the continuous mining machine. At the end of the shift, the continuous mining machine was parked in the second to last crosscut between the Submains Nos. 1 and 2 entries.

At approximately 3:00 p.m., the second shift $003 \mathrm{MMU}$ crew entered the mine and traveled to the section. Les Jarvis, second shift continuous mining machine operator, checked the continuous mining machine and then mined alternating left and right lifts (C) from the Submains No. 1 entry. The MRS units were moved as the lift sequence advanced. At about the time that the second lift was being mined, Niel Baker, shuttle car operator, noticed that the roof was working in the Submains No.1 entry, outby the intersection with the No. 3 entry of the 1 Left Panel. He informed Blake Johnson, MRS operator/roof bolting machine operator, of the condition.

Johnson began checking test holes in the intersection. He checked two 6-foot test holes by inserting a metal tape into the hole and no hazards were detected. He then checked a 12 foot test hole in the affected intersection and found a separation at 11 feet and 5 inches, where his tape would hang on a crack. Johnson told Russell about the crack. Russell checked the 12-foot test hole, but took no corrective action and made no further mention to Johnson on the matter. Johnson continued checking to see if the separation was widening after every two or three shuttle cars, by letting the end of the tape hang on the bottom of the separation and then pushing the tape up to the top of the crack. While mining was being conducted on each side of the Submains No. 1 entry, Johnson told Riley Grant, acting second shift mine foreman, about the crack and Grant indicated no concern regarding the condition. Mining continued, alternating between the left and right lifts from the Submains No. 1 entry. The MRS units were moved as each lift (C) was completed.

The continuous mining machine was then positioned in the No. 3 entry of the 1 Left Panel, where the crew mined lifts (D) from the remainder of the pillar to the right of the entry. During this time, the Nos. 1 and 2 MRS units were located in the 1 Left Panel No. 3 entry and were moved outby with each lift, while the Nos. 3 and 4 MRS units were stationary across the Submains No. 1 entry. As the continuous mining machine was backing out of the final cut (D), a piece of draw rock, approximately 18 inches thick by 7 feet long, fell off the machine onto the mine floor of the 1 Left panel No. 3 entry, in front of the No. 1 MRS (which was located on the left side of the entry, adjacent to the solid pillar).

Johnson and Greg Bantwell, shuttle car operator, began moving the Nos. 1 and 2 MRS units forward. They advanced the No.1 MRS to the fallen draw rock. The No. 2 MRS was then moved completely past the No. 1 MRS, to the edge of the intersection. When they then attempted to push the fallen draw rock with the 
No. 1 MRS, the draw rock wedged between the coal rib and the No. 2 MRS track, with the No. 1 MRS still located completely inby the No. 2 MRS.

They next began moving the Nos. 3 and 4 MRS units out of the Submains No. 1 entry. Johnson lowered the No. 3 MRS, moved it forward into the intersection, and spun the unit so that the cable was facing the pillared portion of the 1 Left Panel. At this point, the unit lost traction in loose material on the mine floor. Russell started the No. 3 MRS on manual and freed the unit. Bantwell then took remote control of the No. 3 MRS and moved it to the midpoint of the crosscut between the Submains Nos. 1 and 2 entries. Johnson then lowered the No. 4 MRS and moved it into the intersection. He spun the MRS and began moving it into the crosscut behind the No. 3 MRS. McCoy and Baker met Bantwell and Johnson in the crosscut to help them continue moving the Nos. 3 and 4 MRS units.

At this time, 9:30 p.m., Russell and Bradford were standing beside the No. 2 MRS, with Russell at the manual controls, when the mine roof fell. The fall extended from the front of the No. 1 MRS, through the intersection, and 13 feet into the crosscut to the back of the No. 4 MRS. Bantwell looked toward Russell and Bradford as the MRS lights disappeared beneath the falling rock.

Johnson, Bantwell, McCoy, and Baker ran out of the crosscut. Suspended dust from the roof fall limited their visibility. They yelled for Russell and Bradford, but heard no response. Noise from the still operating No. 2 MRS hydraulic motor hampered their ability to listen for Russell and Bradford. Bantwell went to the mine telephone and called for help. Johnson tried to start the No. 4 MRS to raise it against the mine roof, but the power for the No. 4 MRS was deenergized. He then tried to shut off the No. 2 MRS by remote control, but it would not stop. Jarvis went to the power center and de-energized the breaker, which shut off the No. 2 MRS. Johnson, McCoy, and Jarvis then went around the pillar to check for Russell and Bradford. However, the roof fall blocked all access to the intersection. There was still no sign of Russell and Bradford.

\section{RECOVERY ACTIVITIES}

Grant and Steve Irwill, third shift outby foreman, arrived on the $003 \mathrm{MMU}$ and spoke with the section crew to determine the last known locations of Russell and Bradford. They directed the men to set the No. 4 MRS at its current location in the Submains crosscut. They then directed the miners to move the No. 3 MRS to the Submains No. 1 entry, at the approach to the roof fall nearest to Russell and Bradford, and set it up, along with additional timbers. Miners deemed nonessential to the recovery activities were sent to the surface.

At 10:30 p.m., Daniel Johnson, Supervisor at MSHA's Harlan Field Office, was informed of the accident. Johnson immediately contacted John Pyles, Assistant District Manager (Enforcement), and Robert W. Rhea, Harlan Field Office Supervisor. Pyles immediately contacted Norman G. Page, District Manager, 
informing him of the accident. Johnson and Pyles then made other phone calls to have recovery teams, investigation teams and a central command setup in the District 7 Barbourville Office.

Johnson arrived at the mine site and was joined by the mine superintendent. Johnson verbally issued a Section 103(k) order and discussed, with the superintendent, the actions that needed to be taken immediately. They discussed notifying families, establishing a command center, setting up security, getting roof support material under ground, getting transportation for recovery efforts, utilizing all available man power in the recovery effort, getting non-essential personnel outside, and establishing a check system for recovery personnel entering and exiting mine. When Darlas Day, Coal Mine Inspector, arrived at the mine, he and Johnson entered the mine with Office of Mine Safety and Licensing (OMSL) inspectors George Johnson and Daven Hoskins.

A recovery plan was developed by the mine operator, as required by Section 103(k) of the Mine Act, and approved by MSHA and OMSL. The recovery began by setting additional supports in the Submains No. 1 entry and utilizing a scoop to remove the rock. At 3:25 a.m. on August 4, 2005, Bradford was located, recovered, and transported to the surface. He was pronounced dead by the Deputy Coroner at 5:30 a.m., as the recovery workers continued searching for Russell. At 6:15 a.m., a second roof fall occurred, extending approximately 25 feet into the recovery area, injuring two recovery personnel, Daven Hoskins, OMSL Inspector, and Shane McConnel, third shift mine foreman. McConnel was operating the scoop at the time of the fall.

A revised recovery plan was developed by the mine operator and approved by MSHA and OMSL. This plan provided for recovery through the crosscut between the Submains Nos. 1 and 2 entries. The recovery activities resumed, and continued non-stop, until 5:00 a.m. on August 7, 2005, when Russell was located, recovered, and transported to the surface. Russell was pronounced dead by the Deputy Coroner at 7:18 a.m.

\section{INVESTIGATION OF THE ACCIDENT}

The investigation was conducted in cooperation with OMSL. A list of those persons who participated in the investigation is contained in Appendix $A$ of this report. Investigation team members traveled to the mine on August 4, 2005, reviewed the roof control plans and maps, and gathered information about the accident. The underground portion of the investigation commenced on August 7,2005 , upon completion of recovery activities. The team traveled underground where measurements, photographs, and mapping were conducted of the 003 MMU. Other documents and relevant information were gathered by the team. A spot inspection was conducted concurrently with the investigation to address enforcement issues that did not contribute to the accident. On August 9, 2005, Combs began a review of the training at the mine. Persons having knowledge 
of the facts regarding the accident were interviewed by MSHA and OMSL on August 9 and 11, 2005.

\section{DISCUSSION}

\section{Geologic Conditions}

The immediate roof on the $003 \mathrm{MMU}$ was comprised of thinly to thickly bedded shale and siltstone. Reportedly, the bolted portion of the immediate roof remained somewhat intact in the fall and had to be cut with the continuous mining machine during recovery operations. Overlying rock layers observed during the investigation had fallen in slabs ranging in thickness between 2 and 16 inches. However, the extensive nature of the roof falls and the recovery/rehabilitation work, along with the proximity of the pillar line, prevented detailed observations of much of the area immediately surrounding the fall site.

The mining height at the fall site was approximately 96 inches.

The closest known core hole to the fall site is BU4807C, located approximately 1,200 feet to the northeast. The core log for this hole describes the rock sequence in ascending order directly above the Lower Harlan coal seam as follows:

- $\quad 1.92$ feet of dark shale

- $\quad 25.42$ feet of sandy, grey shale

- 4.50 feet of grey shale

- $\quad 1.67$ feet of dark shale

The overburden depth at the site of the accident is approximately 1,820 feet. Maps provided by the operator show over-mining of the Upper Harlan and Lower Harlan seams within the boundaries of the mine reserve. Although there are a total of five different mines (only one of which is active) above the active mine reserve, only two of these mines encroach upon the area nearby or directly over the accident site. The abandoned High Splint Mine workings are approximately 1,500 feet above the fall site. A High Splint Mine barrier pillar, approximately 200 feet wide, is situated directly over the site of the accident. The overburden depth at the elevation of the High Splint Mine workings is approximately 300 feet. The second abandoned mine, which appears directly over the fall site, is the Darby Mine. The log from core hole BU4807C, located approximately 1,200 feet northeast of the fall site, shows the Darby Mine workings separated from the Lower Harlan by approximately 175 feet. The available overlay map shows the fall site to be directly under an extensive pillared area in the Darby Mine.

A complete list of seams mined above the mine is listed below. There are no mine workings below this mine.

- Kellioka seam abandoned workings present approximately 155 feet above; 
- $\quad$ Darby seam abandoned workings present approximately 175 feet above;

- Owl seam both abandoned and active workings approximately 235 feet above;

- $\quad \mathrm{F}$ seam both abandoned and inactive workings approximately 355 feet above;

- High Splint seam abandoned workings approximately 1,500 feet above.

Ground conditions in the four-entry Submains were evaluated for three crosscuts outby the fall. Observations in this area revealed the presence of several minor roof cutters and pot-outs. Substantial differences were noted in the degree of rib sloughage between the entries and the crosscuts, apparently associated with cleat orientation. The deterioration seen on the entry ribs was minimal and limited to corner sloughing or light perimeter sloughing.

\section{Roof Control Practices}

The accident site was developed between June 22, 2005 and June 28, 2005. Pillar recovery was conducted over the entire length of the four-entry 1 Left Panel, a distance of 875 feet. The coal pillars surrounding the accident site were two different sizes. The pillars in the panel were nominally 90 feet by 70 feet and the pillars of the mains were 90 feet by 80 feet with an overburden depth of approximately 1,820 feet. The width of the crosscut and Submains No. 1 entry immediately outby the roof fall was approximately 20 feet.

The mine roof was supported on advance with 3/4-inch $\times 48$-inch, grade 40 , fully grouted rebar bolts on four feet $x$ four feet spacing. Also there were five, 0.6-inch diameter, 10-foot cable bolts installed in the intersection with three feet of resin at the anchor point. The cable bolts were installed one on each corner of the intersection and one in the middle of the intersection. Test holes were drilled in accordance with the approved Roof Control Plan in the intersection of each entry to a depth of 12 feet ( 2 feet deeper than the cable bolts) to make roof evaluations for the purpose of pillar recovery. The failure plane occurred at or slightly (up to two feet) above the cable bolt anchorage, which corresponded to the separation detected by Johnson in the test hole at the accident site.

Pillar recovery was accomplished using four MRS units and a 'Christmas tree' or 'twinning' extraction sequence in which alternating left and right lifts are mined from the entries and final lifts are mined from the crosscut. Prior to the accident, six rows of pillars (a total of 18 individual pillars) and the right barrier off the 1 Left No. 4 entry were recovered. Lift Sequence

Mine management intended that pillar recovery be conducted from left to right across the submains following completion of the sixth row of pillars in the 1 Left Panel. The mine superintendent discussed the intended lift sequence with Dotson, including how to make the 90 degree change of direction in pillaring, on the day of the accident, prior to the first shift beginning. However, at the beginning of the first shift, Dotson decided to mine right to left across the $1 \mathrm{Left}$ 
Panel. Once the first lift was taken from the left side of the No. 4 entry, the intended left to right cut sequence across the Submains could not be conducted. Later that shift, the superintendent visited the $003 \mathrm{MMU}$ and saw that lifts had been mined on the left side of the No. 4 panel entry. He then instructed them to stop taking lifts out of that pillar. At 3:05 p.m., Dotson called out a pre-shift examination to Russell, which reported no hazards. They did not discuss which lifts he had mined or which pillar plan (right to left) was being utilized. The superintendent also did not inform the second shift about the lifts being taken out of the pillar. When the second shift began mining, they started using the left to right pillar plan across the Submains, as originally intended by mine management. Mine management did not distribute plans showing the intended cut sequence in writing for reference on the section, nor did they change the cut sequence once the superintendent recognized that the intended cut sequence had been compromised.

\section{Mining Methods}

While mining the last lifts out of the pillar, miners were exposed to faulty pillar recovery methods. There were two roadways being utilized when mining the last lifts from the pillar. After the last lift was taken from the right side of the Submains No. 1 entry, the crosscut should have been blocked by the No. 3 and 4 MRS units and only the roadway through the Submains No. 1 entry used. The continuous mining machine cable was also located through the crosscut, which was located adjacent to a pillar that had been reduced in size with no additional roof support provided.

\section{Mobile Roof Supports}

The four MRS units on the $003 \mathrm{MMU}$ were rated as having a capacity of 600 tons and could be extended to a maximum height of 111 inches. Two radio remote control units and two umbilical cord control units were available on the section to remotely operate the MRS units. The radio remote control units were powered by disposable 9 -volt batteries and had an effective operational range of 200-300 feet.

Each MRS unit is equipped with (hands on) or onboard manual controls which are intended for use by maintenance personnel when servicing the units. The onboard controls are enclosed on the machine frame and are accessed by opening a (flip-up) door. When the MRS units are operated utilizing the onboard manual controls, the signal from the remote control units is overridden. This is consistent with the No. 2 MRS not responding to signals from the remote control following the accident because Russell was manually operating the unit.

The approved Roof Control Plan required MRS units to be moved sequentially in pairs so that each unit would not be offset more than one half unit length from its companion unit. However, this provision was not being complied with at the time of the accident. To comply with the plan, the No. 2 MRS should not have been moved past the No. 1 MRS and the Nos. 3 and 4 MRS units should have 
remained set until the Nos. 1 and 2 MRS units were brought forward into the intersection. Also, the MRS operators were not in a remote location while moving the MRS units, they were in the intersection. This exposed Russell and Bradford to hazards associated with inadequately supported roof immediately adjacent to the incorrectly positioned MRS units and faulty cut sequence.

\section{Training}

Interview statements indicated that mine management did not ensure that the miners understood the lift sequences to be taken while performing the 90 degree change in direction of pillaring or of their normal pillar plan. Mine management did not ensure that the miners possessed an understanding of how the mobile roof supports (MRS) were to be positioned or the miner's location when moving the MRS units. During interviews, miners indicated that they would enter the intersection when lowering the units and would sometimes walk as close as five to six feet from the units when lowering them. They also spun the units around so the cable reel was pointed towards the pillared area and they would position themselves between the MRS unit and the pillared area to watch the MRS unit cable. They discussed moving the MRS units manually when they moved them to the next area to be mined.

Russell was not task trained in the proper operation of the MRS units being used on the $003 \mathrm{MMU}$. No record could be provided to indicate that task training had been conducted in the previous 12 months.

\section{Hazardous Conditions; Posting, Correcting and Recording}

Under the provisions of 30 CFR 75.363 , any hazardous condition found by the mine foreman or equivalent mine official, assistant mine foreman or equivalent mine official, or other certified persons designated by the operator for the purposes of conducting examinations, shall be posted with a conspicuous danger sign where anyone entering the areas would pass. A hazardous condition shall be corrected immediately or the area shall remain posted until the hazardous condition is corrected.

Mine management failed to post the area where a separation had been detected in a roof test hole with a conspicuous danger sign or take corrective action.

Grant and Russell knew about the separation at 11 feet and 5 inches, in the intersection. Johnson informed Russell about the separation and this was confirmed by a witness. Also a different witness confirmed that Johnson told Grant about the separation.

\section{ROOT CAUSE ANALYSIS}

An analysis was conducted to identify the most basic causes of the accident that were correctable through reasonable management controls. During the analysis, causal factors were identified that, if eliminated, would have either 
prevented the accident or mitigated its consequences. Listed below are causal factors identified during the analysis and their corresponding corrective actions implemented to prevent a recurrence of the accident:

1. Causal Factor: Established standards, policies and administrative controls were not being followed in that provisions in the approved Roof Control Plan regarding mining sequence and MRS operation were not being followed.

Corrective Action: The operator ceased retreat mining and provisions that had permitted pillar recovery were removed from the approved Roof Control Plan. The operator developed an Action Plan to address the lack of communication between mine management personnel.

2. Causal Factor: Mine management failed to train all persons in the proper pillar lift sequence and operation of the MRS units.

Corrective Action: The operator developed and implemented an Action Plan that ensured that all miners were trained in all aspects of the Roof Control and Ventilation Plans before returning to mining operations.

3. Causal Factor: Mine management failed to take corrective action when a separation was found in a 12-foot test hole at 11 feet and 5 inches. Mine management allowed mining operations to continue through the intersection in which the roof fall occurred.

Corrective Action: Mine management shall reinforce the importance of recognizing and taking corrective actions when hazards are encountered. Mine management shall develop procedures to prevent miners from being exposed to hazardous conditions such as inadequately supported mine roof.

4. Causal Factor: Mine management exposed miners to the hazards of faulty pillar recovery methods that caused miners to work inby the pillared area of the submains to take the final lifts of the pillar.

Corrective Action: Mine management shall develop procedures to ensure that unsafe pillar recovery methods are not performed.

5. Causal Factor: Mine management failed to ensure that miners are properly task trained in the proper operation of the MRS units.

Corrective Action: Mine management properly task trained miners in the operation of MRS units. Mine management reviewed task training procedures and developed a system to ensure that all miners are properly task trained prior to operating equipment.

6. Causal Factor: Mine management failed to correct the hazard presented by 
the separation at the intersection, or post a conspicuous danger sign to prevent miners from being exposed to hazards at the intersection where the separation was found at 11 feet 5 inches, and where the lifts were taken out of the backside of the pillar.

Corrective Action: Mine management will ensure that certified persons know and understand the hazards presented by similar separations. Mine management will ensure that certified persons who find hazardous conditions post a conspicuous danger sign to warn miners about the hazards.

\section{CONCLUSION}

The accident occurred because of a confluence of factors. The lift sequence for extraction of pillars in the approved Roof Control Plan was not complied with on the $003 \mathrm{MMU}$. Mine management failed to comply with additional safety precautions for the use of MRS units contained in the approved Roof Control Plan while retreat mining was being performed. Mine management failed to adequately train all personnel working on the $003 \mathrm{MMU}$ in pillar recovery methods while utilizing MRS systems. Mine management failed to adequately support the roof where persons were required to work or travel following the detection of a separation in the mine roof, at 11 feet 5 inches up into the roof in the intersection which collapsed resulting in the fatal injuries. Mine management failed to correct the hazard presented by the separation or to post the intersection with a conspicuous danger sign to prevent miners from entering the area and being exposed to a hazard. Mine management exposed miners to hazards related to faulty pillar recovery methods on the $003 \mathrm{MMU}$ by having miners travel inby an area of second mining. Mine management failed to ensure that all personnel were task trained in the operation of MRS units.

\section{ENFORCEMENT ACTIONS}

1. A 103 (K) Order, No. 7540190 was issued to ensure the safety of all persons until an investigation was completed and the area deemed safe.

2. A 104 (d) (2) Order, S\&S, High negligence, No. 7554241, was issued for a violation of 75.220(a)(1): Mine management was not complying with the approved Roof Control Plan when retreat mining (pillaring) was being performed on the $003 \mathrm{MMU}$. The lift sequence approved in the plan was not being followed when mining the last pillar prior to a roof fall occurring which resulted in fatal injuries to two miners. This resulted in improperly placed MRS units and caused miners to work and travel in inadequately supported approaches to the adjacent pillared area.

The approved Roof Control Plan permits only one roadway to the final lifts during retreat mining. However, mine management allowed the use of two roadways to mine the final lifts on the last pillar. 
Information was obtained by an on-site investigation and interviews conducted during a fatal accident investigation.

3. A 104 (d) (2) Order, S\&S, Reckless Disregard negligence, No. 7554242, was issued for a violation of 75.220(a)(1): Mine management was not complying with the additional safety precautions required by the approved Roof Control Plan while using Mobile Roof Support (MRS) units to perform retreat mining on the $003 \mathrm{MMU}$.

Employees operating the MRS units were not positioned in a remote location while the units were being moved to another area. The miners were located in the intersection adjacent to where pillars had been partially removed while moving the Nos. 3 and 4 MRS units.

The No. 1 MRS was not positioned as required by the plan. The No. 1 MRS was positioned behind the No. 2 MRS.

The No. 2 MRS was being operated manually prior to the fall. The MRS units had been operated manually on prior occasions. The No. 3 MRS had been operated manually during the shift when it became stuck. The first shift foreman also admitted manually operating a MRS on other occasions during retreat mining.

Information was obtained by an on-site investigation and interviews conducted during a fatal accident investigation.

4. A 104 (d) (2) Order, S\&S, High negligence, No. 7554243, was issued for a violation of 75.220(a) (1): Mine management failed to adequately train all personnel working on the $003 \mathrm{MMU}$ in pillar recovery methods when Mobile Roof Support (MRS) units were being used. When interviewed, miners from both shifts on the $003 \mathrm{MMU}$ did not exhibit a clear understanding of the requirements of the approved Roof Control Plan, including the lift sequence when mining pillars, the proper location for miners when the MRS units are being moved, and that the MRS units should only be operated by the remote control unit while second mining of pillars.

Information was obtained by an on-site investigation and interviews conducted during a fatal accident investigation.

5. A 104 (d) (2) Order, S\&S, Reckless Disregard negligence, No. 7554244, was issued for a violation of 75.202(a): Mine management failed to adequately support or otherwise control the roof where persons were required to work or travel. Two miners were moving Mobile Roof Support (MRS) units on the 003 MMU when a roof fall occurred which resulted in the death of both miners. Both miners were located in close proximity to the MRS units being moved. Also, the two remaining MRS units on the section had been removed from the approach 
to the adjacent pillared area, at the intersection immediately outby the location where the miners were working. Prior to the accident, mine management was aware of a separation in the mine roof that was detected in a 12-foot test hole at 11 feet 5 inches in the intersection from which the MRS units were removed, which was also where the roof fall occurred. The roof in the intersection was supported by 4 -foot fully grouted roof bolts and 10 -foot cable bolts. Additional roof support was not installed or other action taken to assure persons were protected from hazards related to falls of the roof after the separation was detected.

Information was obtained by an on-site investigation and interviews conducted during a fatal accident investigation.

6. A 104 (d) (2) Order, S\&S, Reckless Disregard negligence, No. 7554245, was issued for a violation of 75.363(a): Hazardous conditions found and known by mine management were not immediately corrected and the area was not posted with a conspicuous danger sign until the hazardous conditions were corrected. During the second shift, two members of mine management were informed that a separation at 11 feet 5 inches existed in the test hole located in the intersection where the roof fall ultimately occurred. However, no corrective action was taken. Pillar mining continued in the area and miners were exposed to the hazardous condition. Moreover, mine management was aware that two MRS units were moved out of the intersection prior to miners continuing to work in the area. The roof fall was approximately 12 feet thick. Two miners suffered fatal injuries as a result of working in the area exposed to these hazardous conditions.

In addition, mine management directed first, second and third shifts to mine the pillars in a left to right sequence across the submains. During the shift prior to the roof fall, mine management observed lifts taken by the first shift crew on the backside corner of the pillar and the adjacent wall. Once taken, the planned left to right sequence of the submains could not be conducted without adequate corrective action. Mine management failed to inform the second shift about the lifts taken on first shift and failed to ensure that adequate corrective action was taken. The second shift crew continued the left to right lift sequence as previously directed by mine management. This increased the hazardous conditions that contributed to the roof fall.

Information was obtained by an on-site investigation and interviews conducted during a fatal accident investigation.

7. A 104 (a) Citation, S\&S, High negligence, No.7554246, was issued for a violation of 48.7: Mine management failed to provide Clyde Russell with task training in the operation of MRS units. Russell suffered fatal injuries when a roof fall occurred while he was manually operating a MRS in an intersection. 
Information was obtained by an on-site investigation and interviews conducted during a fatal accident investigation.

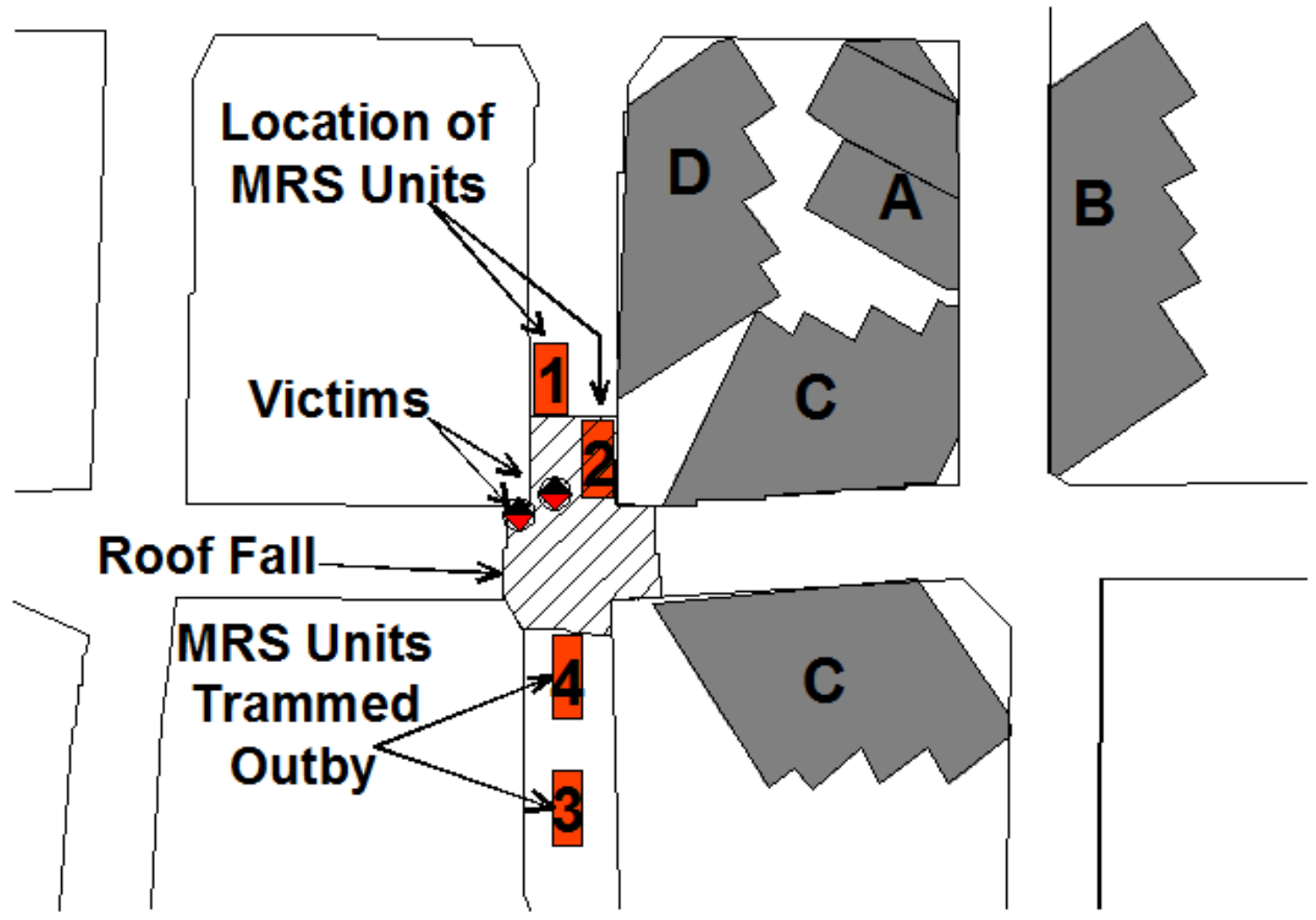

MSHA 2009 


\section{APPENDIX B}

Sampling Forms 


\begin{tabular}{|c|c|c|c|c|}
\hline & & 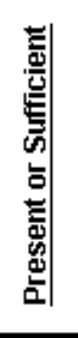 & 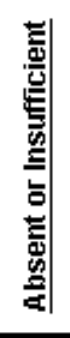 & $\begin{array}{l}\text { Notes (including Probability of Ney ative Consequences) } \\
\qquad(\mathrm{NA}, \mathrm{V}, \mathrm{SL}, \mathrm{P}, \mathrm{U})\end{array}$ \\
\hline \multicolumn{5}{|c|}{\begin{tabular}{l|l} 
Roof Conditions \\
\end{tabular}} \\
\hline \multicolumn{5}{|c|}{\begin{tabular}{l|l}
$\underline{2}$ & Floor Conditions \\
\end{tabular}} \\
\hline \multicolumn{5}{|c|}{\begin{tabular}{l|l}
$\underline{3}$ & Rib Conditions
\end{tabular}} \\
\hline \multicolumn{5}{|c|}{\begin{tabular}{l|l}
4 Overmining \\
\end{tabular}} \\
\hline \multicolumn{5}{|c|}{$\underline{5}$ Undermining } \\
\hline \multicolumn{5}{|c|}{ 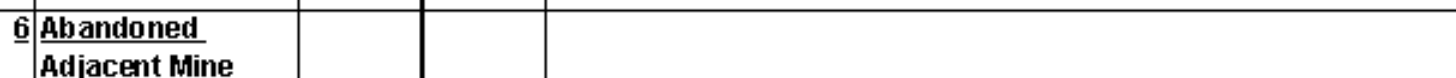 } \\
\hline \multicolumn{5}{|c|}{$\begin{array}{l}7 \\
7 \text { Pillar Dimensions }\end{array}$} \\
\hline \multicolumn{5}{|c|}{ 8 1 Timbering Plan } \\
\hline \multicolumn{5}{|c|}{9 Mobile Roof } \\
\hline \multicolumn{5}{|c|}{10 Depth of Cover } \\
\hline \multicolumn{5}{|c|}{11 Distance from } \\
\hline \multicolumn{5}{|c|}{\begin{tabular}{l|l}
12 & ARMPS Pillar \\
\end{tabular}} \\
\hline \multicolumn{5}{|c|}{13 Ventilation } \\
\hline \multicolumn{5}{|c|}{ 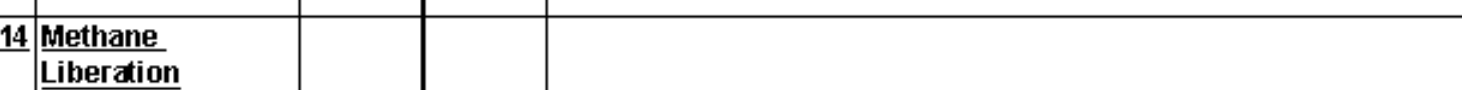 } \\
\hline \multicolumn{5}{|c|}{15} \\
\hline \multicolumn{5}{|l|}{16} \\
\hline \multicolumn{5}{|l|}{17} \\
\hline 18 & & & & \\
\hline & obability: Nearly A & & & ble \\
\hline
\end{tabular}




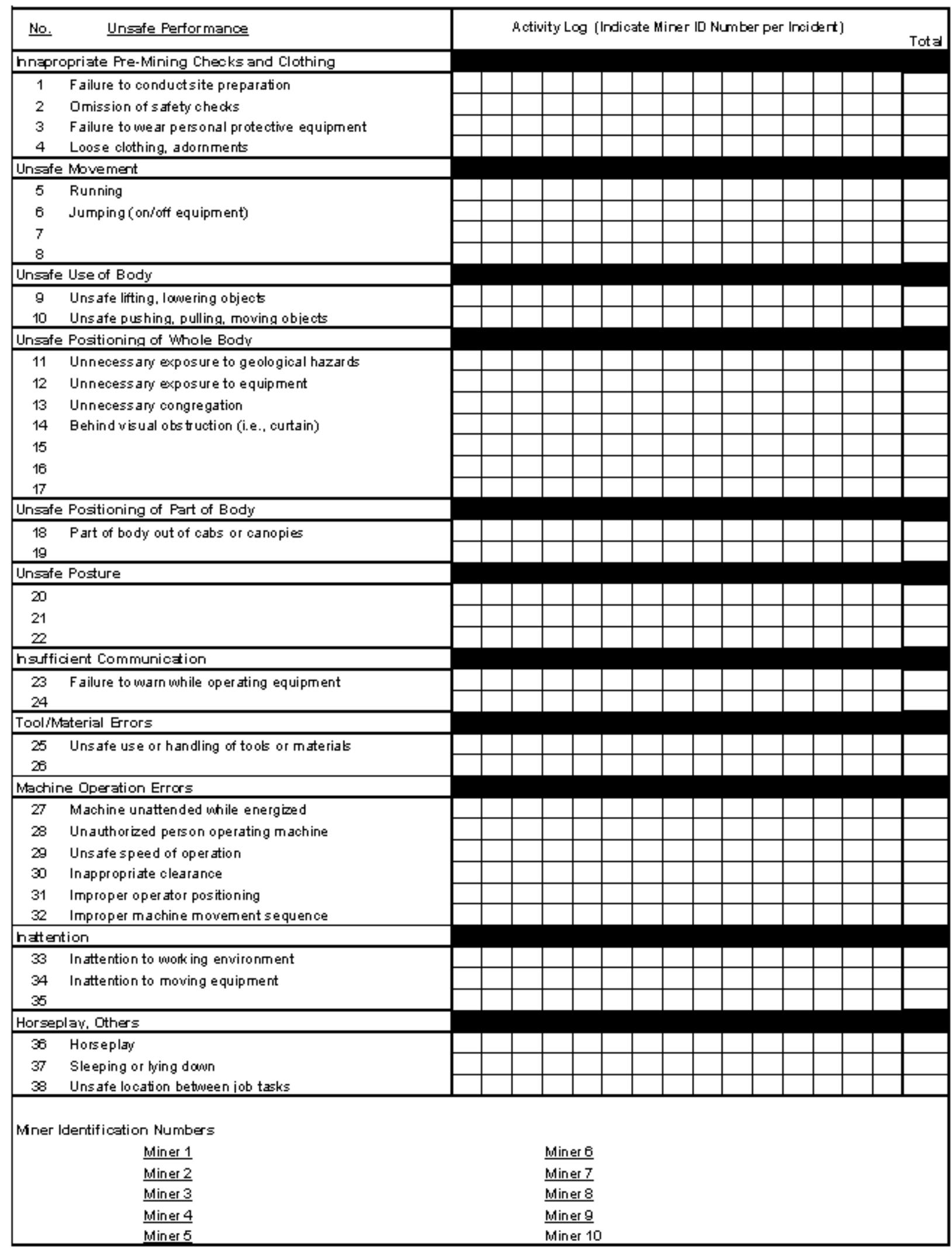




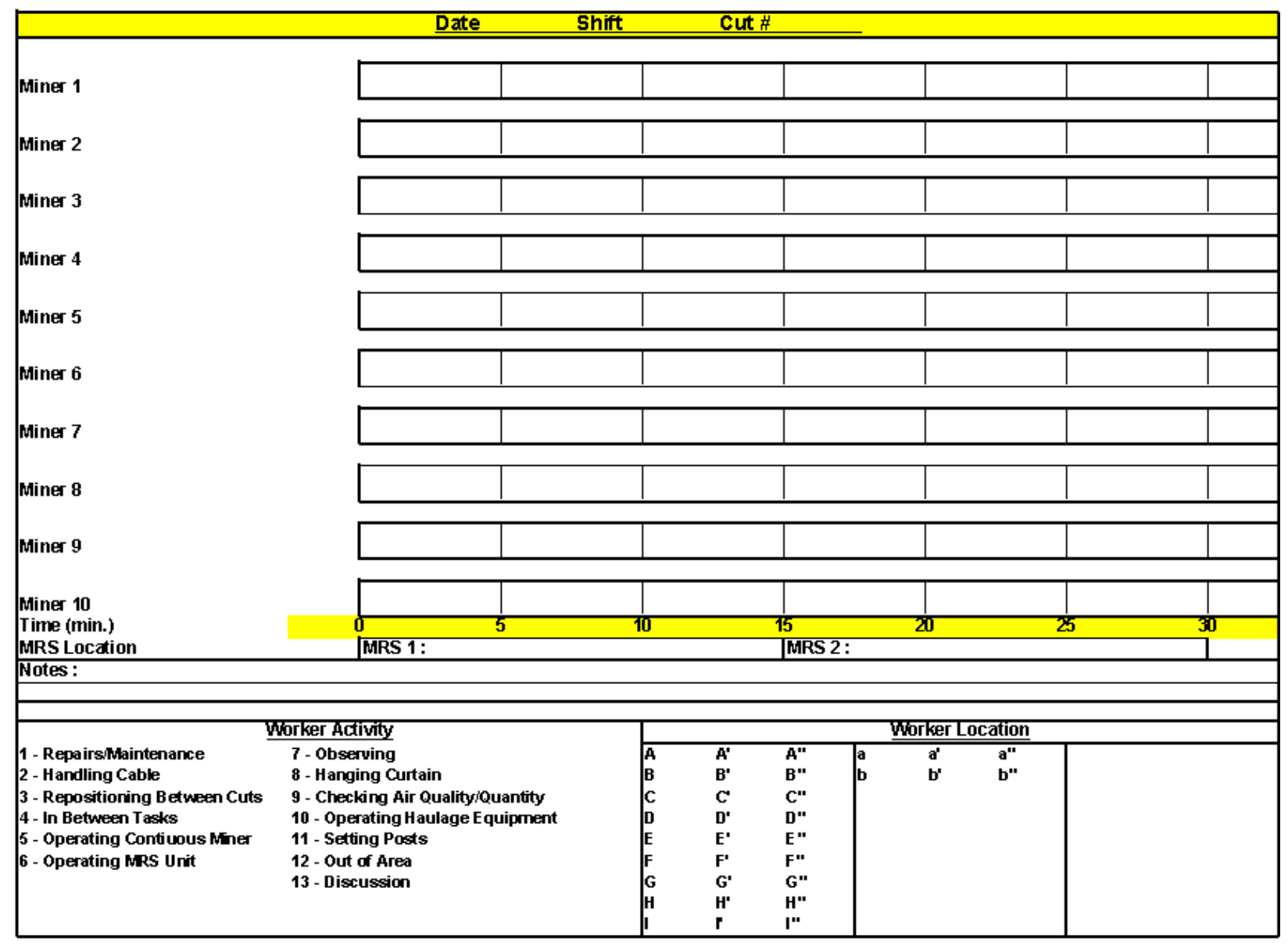




\section{APPENDIX C}

Field Application Originals 


\begin{tabular}{|c|c|c|c|}
\hline & 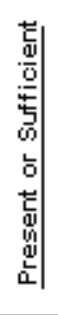 & 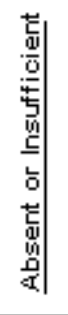 & $\begin{array}{l}\text { Notes (induding Probability of Negative Consequences) } \\
\qquad(N A, V L, S L, P, U)\end{array}$ \\
\hline 1 Roof Conditions & & & Sandstone with Coal Streaks, prone to difficult conditions. (SL) \\
\hline$\underline{2}$ Floor Conditions & & & Good condition, very little mud. (P) \\
\hline 3 Rib Conditions & & & Rib is bolted but deteriorated, post are set to maintain roadna ay widthe (P) \\
\hline 4 Overmining & $\mathrm{P}$ & & Pres ent within approxim ately $60 \mathrm{ft}$ (P) \\
\hline$\underline{5} \underline{\text { Undermining }}$ & $\mathrm{P}$ & & Undermined with in approximately $100 \mathrm{ft}$ (P) \\
\hline$\underline{6} \frac{\text { Abandoned }}{\text { Adjiacent Mine }}$ & & A & 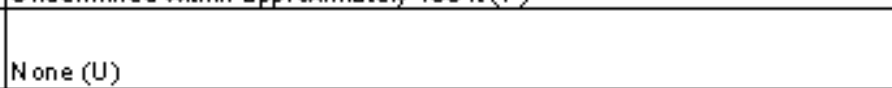 \\
\hline$\frac{7}{\text { Consistency of }}$ & & I & Significant degradation of the pillars has lead to a reduced uniformity (P) \\
\hline$\underline{8}$ Timbering Plan & & A & MRS units used instead of wooden timbers (U) \\
\hline$\frac{9}{\text { Mobile Roof }}$ & $\mathrm{P}$ & & MRS PIan (U) \\
\hline 10 Depth of Cover & & & 800 to $1000 \mathrm{ft}(\mathrm{P})$ \\
\hline 11 Distancefrom & & & Not Known bypers onnel(P) \\
\hline 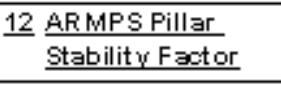 & & A & Not Known bypers onnel(P) \\
\hline 13 Ventilation & $\mathrm{s}$ & & MSHA irs pector verified ventilation compliancy (U) \\
\hline $14 \frac{\text { Methane }}{\text { Liberation }}$ & $\mathrm{P}$ & & Liberation rate not known, but methane levels were sampled regularly (P) \\
\hline$\underline{15}$ & & & \\
\hline$\underline{16}$ & & & \\
\hline 17 & & & \\
\hline$\underline{18}$ & & & \\
\hline ility: $\mathrm{Ne}$ & & . & Likely (VL), Somewhat Likely (SL), Possible (P), Unlikely (U) \\
\hline
\end{tabular}




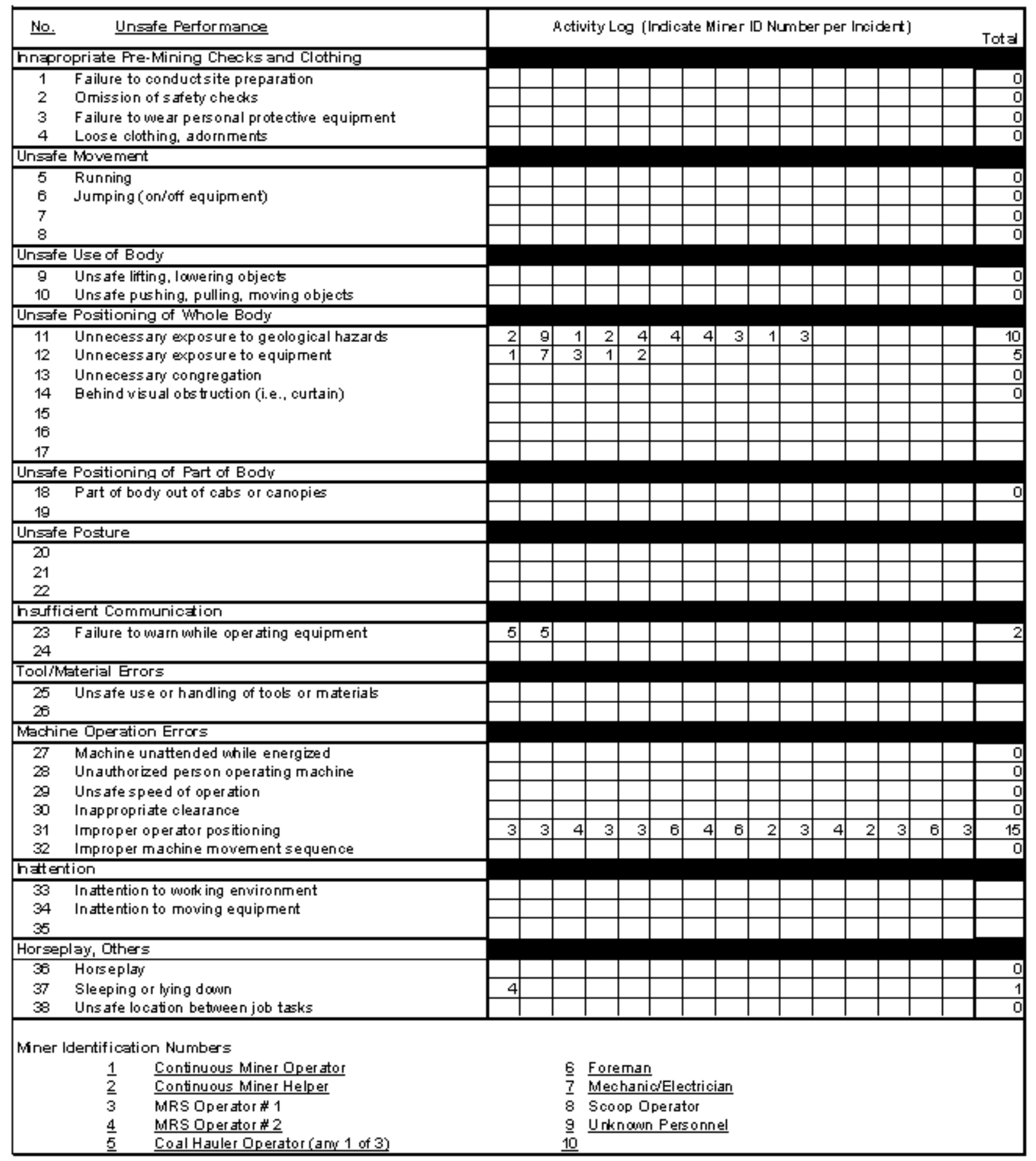




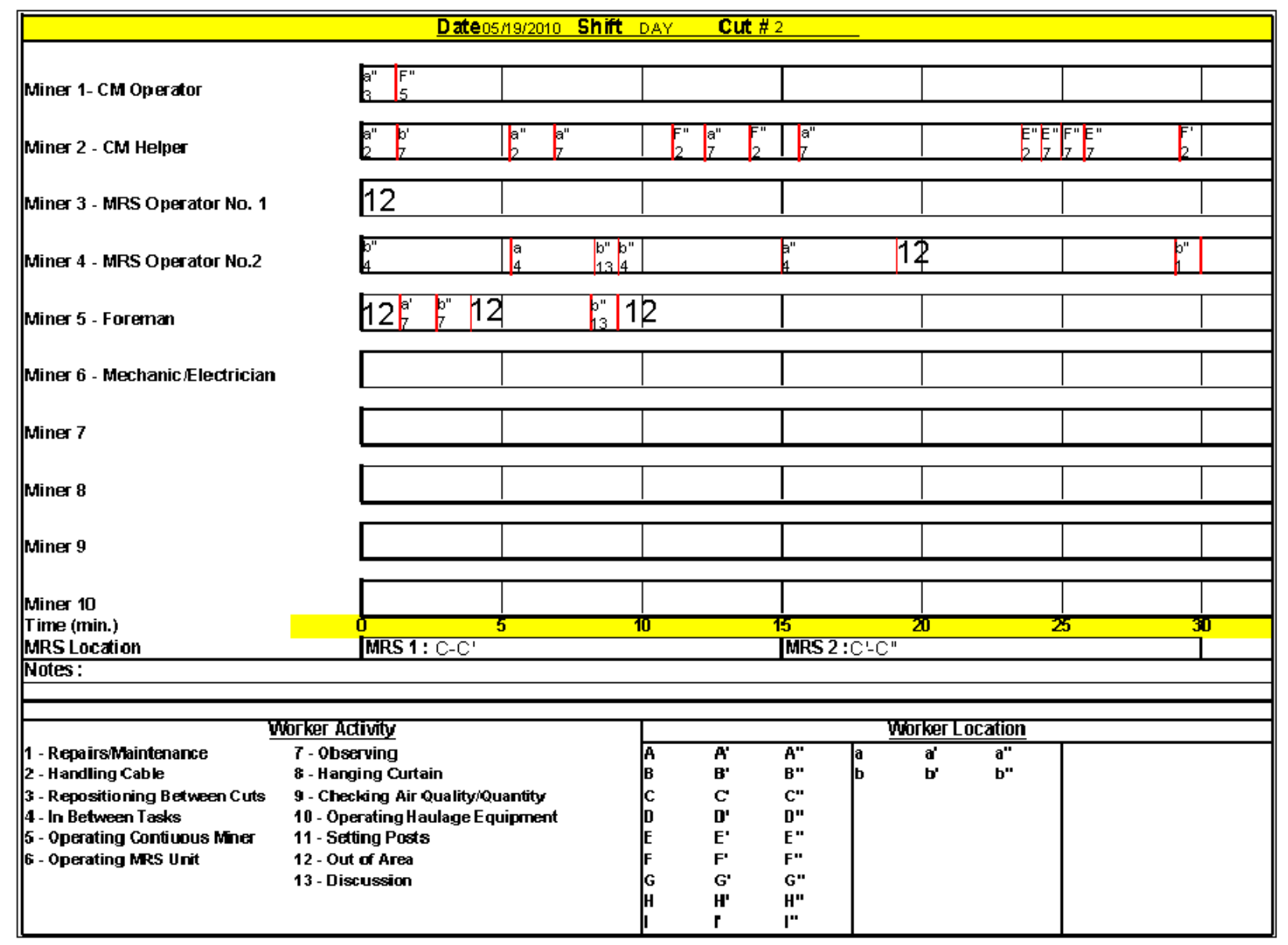




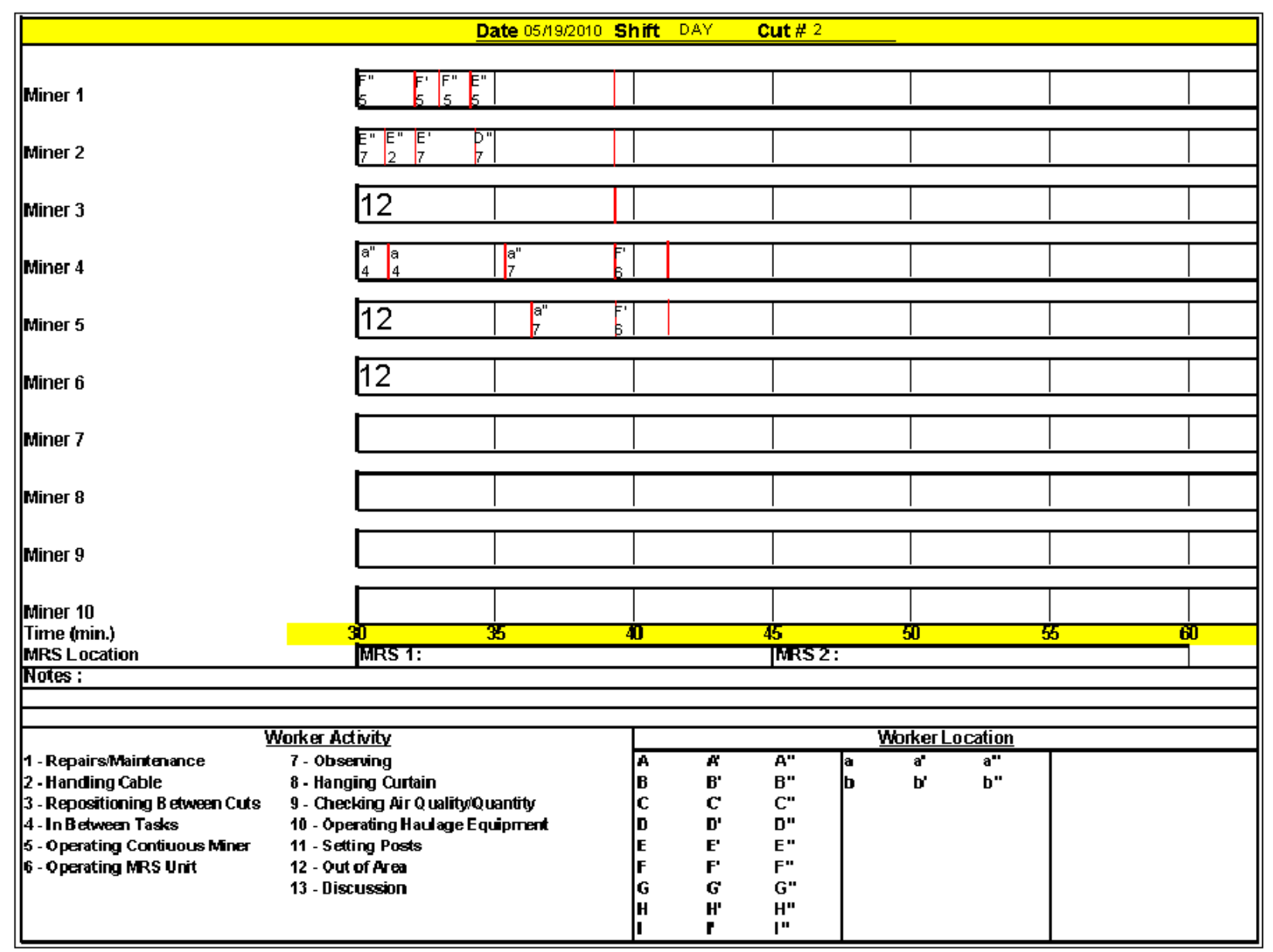




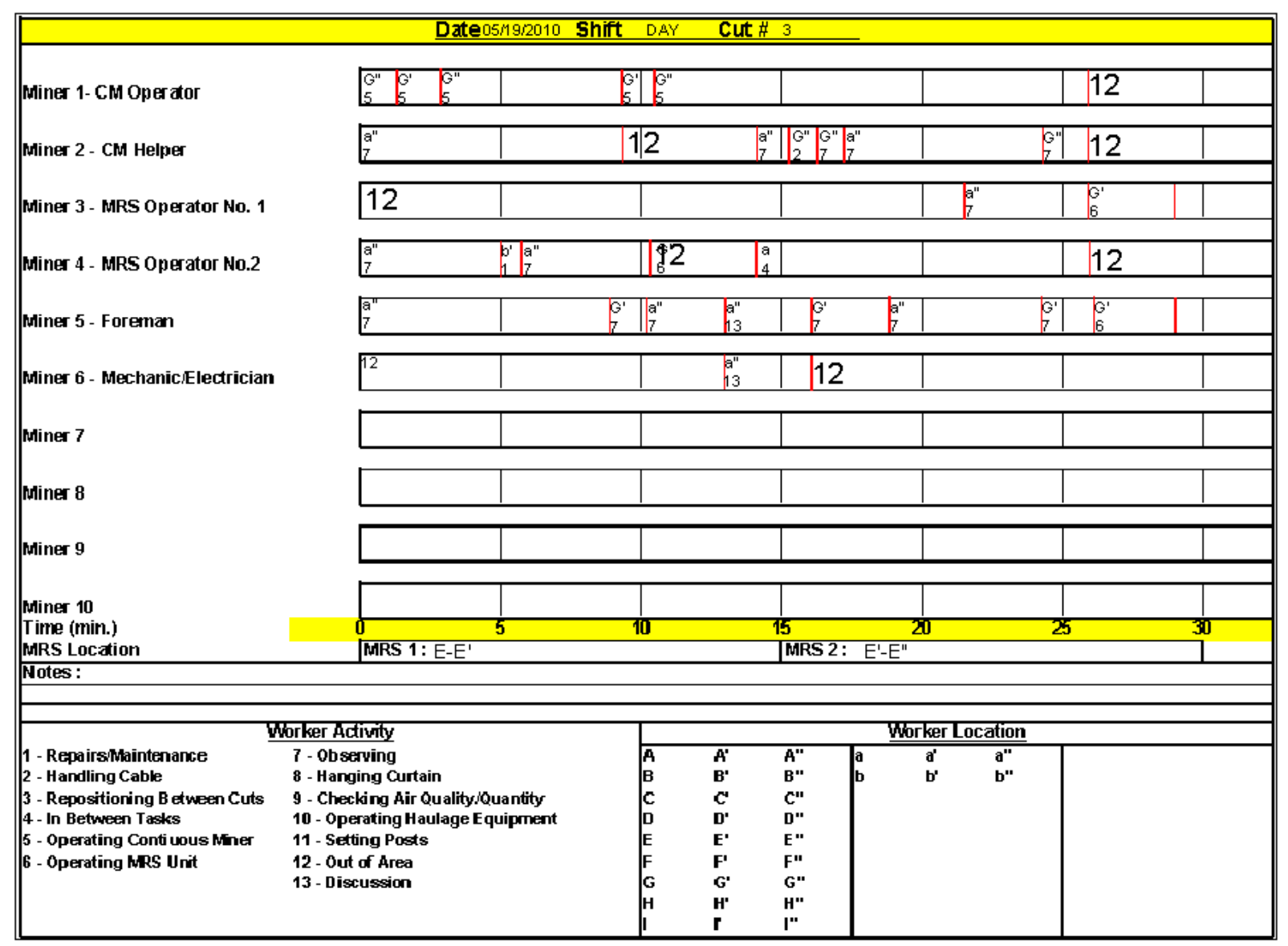

JOhn 